Supporting Information

\title{
Formation of Enamines via Catalytic Dehydrogenation by Pincer-Iridium Complexes
}

Yansong J. Lu, ${ }^{+}$Xiawei Zhang, ${ }^{\dagger}$ Santanu Malakar, ${ }^{+}$Karsten Krogh-Jespersen, ${ }^{\dagger}$ Faraj Hasanayn, ${ }^{\ddagger}$ Alan S. Goldman*,+

${ }^{\dagger}$ Department of Chemistry and Chemical Biology, Rutgers, The State University of New Jersey, New Brunswick, New Jersey 08903, United States

*E-mail: alan.goldman@rutgers.edu

‡Department of Chemistry, American University of Beirut, Beirut 1107 2020, Lebanon

List of Contents

1. Computational Details $\quad$ S2

2. Energies of intermediates and transition states (Tables S1-S7) S3

3. Kinetic Isotope Effect Calculations (Tables S8-S9) S9

4. References $\quad$ S10

5. Cartesian coordinates of DFT optimizations S11

6. NMR spectra of unreported amines and reaction mixtures $\quad$ S85 


\section{Computational Details}

All electronic structure calculations employed the DFT method, ${ }^{\mathrm{S} 1}$ the $\mathrm{M} 06$ functional, ${ }^{\mathrm{S} 2}$ and the SMD dielectric continuum solvation model with $p$-xylene as model solvent. ${ }^{\mathrm{S3}}$ For Ir, we applied the SDD relativistic effective (small) core potential and associated $(6 s 5 p 3 d)$ valence basis set; 54 all other atoms $(P, N, C$, and $H)$ were assigned allelectron $6-31 G(d, p)$ basis sets. ${ }^{55}$

Geometries were calculated for stationary points along the reaction paths using standard optimization procedures. $^{\mathrm{S6}}$ In all geometry optimizations involving amines, a superfine integration grid size was used, whereas for alkanes the default ultrafine grid size was employed. Tighter optimization convergence criteria were used for the amines ( ${ }^{2} \mathrm{Pr}_{2} \mathrm{NEt}, \mathrm{Me}_{2} \mathrm{NEt}$ and $\mathrm{N}$-methylpiperidine), whereas default convergence was satisfactory for the alkanes ( ${ }^{P_{2}} \mathrm{C}(\mathrm{H}) \mathrm{Et}, \mathrm{Me}_{2} \mathrm{C}(\mathrm{H}) \mathrm{Et}, \mathrm{COA}$ and $n$-butane). Intrinsic Reaction Coordinate (IRC) calculations were conducted on important transition states to properly locate matching reactants/products. ${ }^{57}$ Normal mode analysis was performed to further verify the nature of a particular stationary point (minimum, transition state). The resulting set of vibrational frequencies was employed (without scaling) to determine zero-point energy corrections.

Thermal correction terms to the Gibbs free energy of each molecule were evaluated using standard statistical mechanics expressions applicable to an ideal gas and assuming harmonic oscillator/rigid rotor approximations, ${ }^{\text {s8 }}$ at $\mathrm{T}=323.15 \mathrm{~K}$ and $\mathrm{P}=1 \mathrm{~atm}$. These energies were converted to standard state free energies defined at $\mathrm{T}=323.15 \mathrm{~K}$ and $1.0 \mathrm{M}$ concentration by adding a term of $2.10 \mathrm{kcal} / \mathrm{mol}$. We note, from a simple application of the ideal gas law, that a mole of gas at $\mathrm{P}=1.0 \mathrm{~atm}$ and $\mathrm{T}=323.15 \mathrm{~K}$ corresponds to a concentration of $0.038 \mathrm{M}$. Equivalently, a concentration of $1.0 \mathrm{M}$ at $323.15 \mathrm{~K}$ corresponds to $\mathrm{P}=26.5 \mathrm{~atm}$. Thus, a change from $\mathrm{P}=1.0 \mathrm{~atm}$ to $\mathrm{P}=26.5$ atm at $323.15 \mathrm{~K}$ is accompanied with $\Delta \mathrm{G}=-\mathrm{RT} \ln (1 / 26.5)=2.10 \mathrm{kcal} / \mathrm{mol}$. All Gibbs free energies cited in the manuscript are derived from this procedure, unless noted otherwise. The kinetic isotope effects (KIE) ${ }^{\mathrm{Sg}}$ are reported at $\mathrm{T}=$ $323.15 \mathrm{~K}$ using unscaled vibrational frequencies.

All calculations used methodologies implemented in the Gaussian 16, Rev. A03 program. ${ }^{\mathrm{S} 10}$ The graphical interface GaussView was used to prepare input and analyze output files. ${ }^{\text {S11 }}$

Geometries of principal species are available in .mol format (compacted into a .zip file). For convenience, Scheme 6 in the Text is reproduced below as Scheme S1, respectively, along with energetic quantities in tabular form (Tables S1-S6). Tables S7 and S8 shows the energetics of the KIE calculations at $50^{\circ} \mathrm{C}$ and $90{ }^{\circ} \mathrm{C}$ respectively.

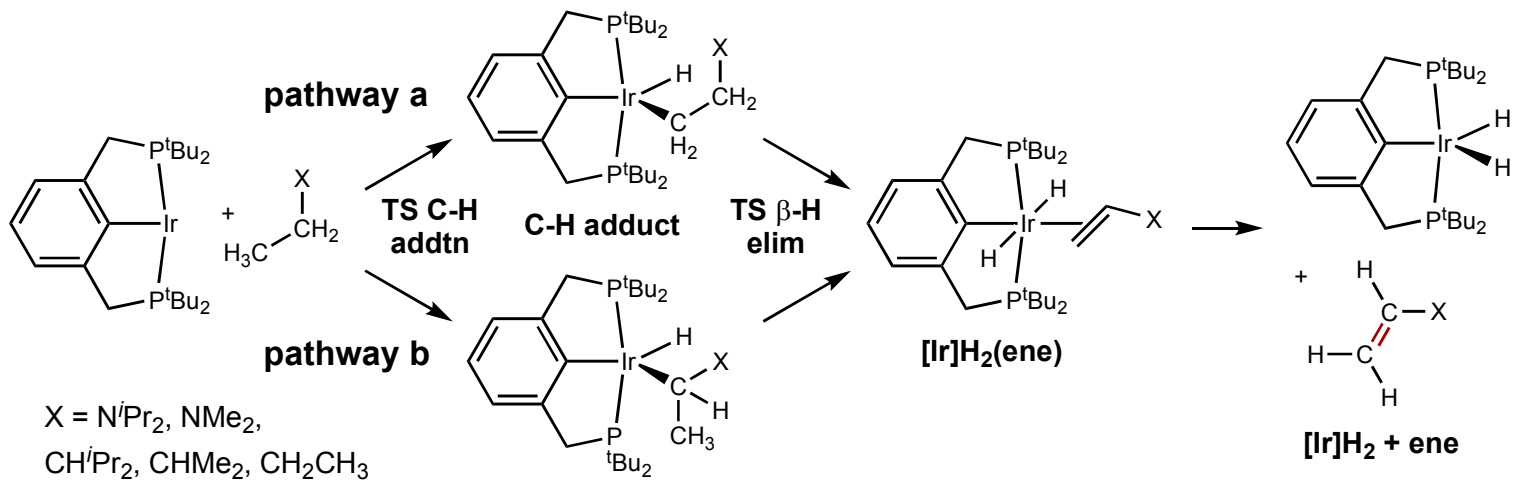

Scheme S1. Putative Pathways for Dehydrogenation of Amines and Alkanes Examined by DFT Calculations 


\section{Energies of intermediates and transition states ( $\mathrm{kcal} / \mathrm{mol})$}

Table S1. Energies of dehydrogenation of $N$-ethyldiisopropylamine by ( $\left.{ }^{\text {Bu }} 4 \mathrm{PCP}\right) \mathrm{Ir}$ referenced to $\mathrm{T}=323.15 \mathrm{~K}\left(50^{\circ} \mathrm{C}\right)(\mathrm{P}=1 \mathrm{~atm})$.

\begin{tabular}{|c|c|c|c|}
\hline Name & Structure & $\Delta \mathrm{E}$ & $\Delta \mathbf{G}$ \\
\hline 1 & ${ }^{i} \mathrm{Pr}_{2} \mathrm{NCH}_{2} \mathrm{CH}_{3}$ & & \\
\hline 2 & ('BuPCP) Ir & & \\
\hline 3 & $1+2$ & 0.0 & 0.0 \\
\hline 4 & $\sigma-\mathrm{C}-\mathrm{H}$ complex & -10.9 & 4.9 \\
\hline \multicolumn{4}{|c|}{ pathway a } \\
\hline TS1 & terminal $\mathrm{C}-\mathrm{H}$ activation & 9.1 & 22.3 \\
\hline 5 & terminal-alkyl-(PCP)|r-complex & 1.7 & 16.3 \\
\hline TS2 & $\begin{array}{c}\beta-\mathrm{H} \text { elimination from internal } \\
\text { position }\end{array}$ & 18.8 & 33.5 \\
\hline \multicolumn{4}{|c|}{ pathway b } \\
\hline TS1' & internal $\mathrm{C}-\mathrm{H}$ activation & 16.1 & 32.1 \\
\hline $5^{\prime}$ & internal-alkyl-(PCP)Ir complex & 13.8 & 31.2 \\
\hline TS2' & $\begin{array}{c}\beta-\mathrm{H} \text { elimination from terminal } \\
\text { position }\end{array}$ & 18.8 & 34.7 \\
\hline 6 & $(\mathrm{PCP}) \operatorname{Ir}(\mathrm{H})_{2}($ enamine $)$ & 6.7 & 22.1 \\
\hline $\begin{array}{c}\text { trans-2- }-\mathrm{H}_{2}+ \\
\text { enamine }\end{array}$ & trans-( $(\mathrm{Bu} P C \mathrm{P}) \mathrm{IrH} \mathrm{H}_{2}+$ enamine & 25.3 & 22.1 \\
\hline cis-2- $\mathrm{H}_{2}+$ enamine & cis-( $\left.{ }^{\mathrm{tBu}} \mathrm{PCP}\right) \mathrm{IrH} \mathrm{H}_{2}+$ enamine & 16.8 & 9.2 \\
\hline enamine & $\mathrm{PP}_{2} \mathrm{NCH}=\mathrm{CH}_{2}$ & & \\
\hline $\mathbf{H}_{2}$ & $\mathrm{H}_{2}+2$ & 0.0 & 0.0 \\
\hline trans-2- $\mathrm{H}_{2}$ & trans-( $\left.{ }^{\mathrm{tBu}} \mathrm{PCP}\right) \mid \mathrm{r} \mathrm{H}_{2}$ & -11.6 & 1.9 \\
\hline cis-2- $\mathrm{H}_{2}$ & cis-( $\left.{ }^{\mathrm{tBu}} \mathrm{PCP}\right) \mathrm{IrH}_{2}$ & -20.1 & -11.0 \\
\hline
\end{tabular}


Table S2. Energies of dehydrogenation of $N$-ethyldimethylamine by ( $\left.{ }^{\text {B }}{ }^{4} \mathrm{PCP}\right) \mathrm{Ir}$ referenced to $\mathrm{T}=323.15 \mathrm{~K}\left(50^{\circ} \mathrm{C}\right)(\mathrm{P}=1 \mathrm{~atm})$.

\begin{tabular}{|c|c|c|c|}
\hline Name & Structure & $\Delta \mathrm{E}$ & $\Delta \mathbf{G}$ \\
\hline 1 & $\mathrm{Me}_{2} \mathrm{NCH}_{2} \mathrm{CH}_{3}$ & & \\
\hline 2 & ( ${ }^{\text {BuuPCP) }}$ ) $r$ & & \\
\hline 3 & $1+2$ & 0.0 & 0.0 \\
\hline 4 & $\sigma-\mathrm{C}-\mathrm{H}$ complex & -9.8 & 6.2 \\
\hline \multicolumn{4}{|c|}{ pathway a } \\
\hline TS1 & terminal $\mathrm{C}-\mathrm{H}$ activation & 8.7 & 21.6 \\
\hline 5 & terminal-alkyl-(PCP)Ir-complex & 1.1 & 15.3 \\
\hline TS2 & $\begin{array}{l}\beta-\mathrm{H} \text { elimination from internal } \\
\text { position }\end{array}$ & 18.9 & 32.9 \\
\hline \multicolumn{4}{|c|}{ pathway b } \\
\hline TS1' & internal $\mathrm{C}-\mathrm{H}$ activation & 12.7 & 27.2 \\
\hline $5^{\prime}$ & internal-alkyl-(PCP)Ir complex & 9.9 & 26.0 \\
\hline TS2' & $\begin{array}{l}\beta \text {-H elimination from terminal } \\
\text { position }\end{array}$ & 19.2 & 34.1 \\
\hline 6 & $(\mathrm{PCP}) \operatorname{Ir}(\mathrm{H})_{2}$ (enamine) & 5.5 & 23.0 \\
\hline trans-2- $\mathrm{H}_{2}+$ enamine & trans-( $\left.{ }^{\mathrm{tBu}} \mathrm{PCP}\right) \mathrm{Ir} \mathrm{H}_{2}+$ enamine & 23.5 & 21.0 \\
\hline cis-2- $\mathrm{H}_{2}+$ enamine & cis-( $\left.{ }^{\mathrm{tBu}} \mathrm{PCP}\right) \mathrm{IrH} \mathrm{H}_{2}+$ enamine & 15.0 & 8.1 \\
\hline enamine & $\mathrm{Me}_{2} \mathrm{NCH}=\mathrm{CH}_{2}$ & & \\
\hline
\end{tabular}


Table S3. Energies of dehydrogenation of $\mathrm{N}$-methylpiperidine by ( $\left.{ }^{\mathrm{tBu} 4} \mathrm{PCP}\right) \mathrm{Ir}$ referenced to $\mathrm{T}=323.15 \mathrm{~K}\left(50^{\circ} \mathrm{C}\right)(\mathrm{P}=1 \mathrm{~atm})$.

\begin{tabular}{|c|c|c|c|}
\hline Name & Structure & $\Delta \mathrm{E}$ & $\Delta \mathbf{G}$ \\
\hline 1 & $N$-methylpiperidine & & \\
\hline 2 & ('BuPCP) Ir & & \\
\hline 3 & $1+2$ & 0.0 & 0.0 \\
\hline \multicolumn{4}{|c|}{ pathway a } \\
\hline TS1 & $\mathrm{C}-\mathrm{H}$ activation at $\beta$-position & 12.4 & 25.9 \\
\hline 5 & $\beta$-alkyl-(PCP)Ir-complex & 6.2 & 20.4 \\
\hline TS2 & $\beta$-H elimination from $\alpha-$ position & 25.3 & 38.8 \\
\hline \multicolumn{4}{|c|}{ pathway b } \\
\hline TS1' & $\mathrm{C}-\mathrm{H}$ activation at $\alpha$-position & 15.8 & 30.9 \\
\hline $5^{\prime}$ & $\alpha$-alkyl-(PCP)|r-complex & 8.3 & 24.9 \\
\hline TS2' & $\beta$-H elimination from $\beta$-position & 29.4 & 43.6 \\
\hline 6 & $(\mathrm{PCP}) \operatorname{Ir}(\mathrm{H})_{2}$ (enamine) & 11.7 & 25.8 \\
\hline $\begin{array}{c}\text { trans-2- }-\mathrm{H}_{2}+ \\
\text { enamine }\end{array}$ & trans-( $\left.{ }^{\mathrm{tBu}} \mathrm{PCP}\right) \mathrm{Ir} \mathrm{H}_{2}+$ enamine & 24.0 & 20.7 \\
\hline cis-2- $\mathrm{H}_{2}+$ enamine & cis-( $\left.{ }^{\text {tBuPCP}}\right) \mid \mathrm{rH}_{2}+$ enamine & 15.4 & 7.7 \\
\hline enamine & $\mathrm{Pr}_{2} \mathrm{NCH}=\mathrm{CH}_{2}$ & & \\
\hline $\mathrm{H}_{2}$ & $\mathrm{H}_{2}+2$ & 0.0 & 0.0 \\
\hline trans-2- $\mathrm{H}_{2}$ & trans-( $\left.{ }^{\mathrm{tBu}} \mathrm{PCP}\right) \mathrm{IrH} \mathrm{H}_{2}$ & -11.6 & 1.9 \\
\hline cis-2- $\mathrm{H}_{2}$ & cis-( $\left.{ }^{\mathrm{tBu}} \mathrm{PCP}\right) \mathrm{IrH}_{2}$ & -20.1 & -11.0 \\
\hline
\end{tabular}


Table S4. Energies of dehydrogenation of 2-methyl-3-isopropylpentane by ( $\left.{ }^{\mathrm{tBu} 4} \mathrm{PCP}\right)$ Ir referenced to $\mathrm{T}=323.15 \mathrm{~K}\left(50^{\circ} \mathrm{C}\right)(\mathrm{P}=1 \mathrm{~atm})$.

\begin{tabular}{|c|c|c|c|}
\hline Name & Structure & $\Delta \mathrm{E}$ & $\Delta \mathbf{G}$ \\
\hline 1 & ${ }^{\mathrm{P}} \mathrm{Pr}_{2} \mathrm{CHCH}_{2} \mathrm{CH}_{2} \mathrm{CH}_{3}$ & & \\
\hline 2 & (tBuPCP)Ir & & \\
\hline 3 & $1+2$ & 0.0 & 0.0 \\
\hline 4 & $\sigma-\mathrm{C}-\mathrm{H}$ complex & -6.7 & 10.6 \\
\hline \multicolumn{4}{|c|}{ pathway a } \\
\hline TS1 & terminal $\mathrm{C}-\mathrm{H}$ activation & 10.5 & 22.8 \\
\hline 5 & terminal-alkyl-(PCP)|r-complex & 4.2 & 19.3 \\
\hline TS2 & $\begin{array}{c}\beta \text {-H elimination from internal } \\
\text { position }\end{array}$ & 23.3 & 38.5 \\
\hline \multicolumn{4}{|c|}{ pathway $b$} \\
\hline aTS1' & internal $\mathrm{C}-\mathrm{H}$ activation & 18.2 & 32.4 \\
\hline $5^{\prime}$ & internal-alkyl-(PCP)Ir complex & 16.2 & 32.1 \\
\hline TS2' & $\begin{array}{l}\beta \text {-H elimination from terminal } \\
\text { position }\end{array}$ & 21.4 & 35.0 \\
\hline 6 & $(\mathrm{PCP}) \operatorname{Ir}(\mathrm{H})_{2}$ (olefin) & 9.4 & 24.6 \\
\hline trans $-2-\mathrm{H}_{2}+$ olefin & trans-('BuPCP) $\mid \mathrm{rH}_{2}+$ olefin & 30.6 & 27.0 \\
\hline cis-2- $\mathrm{H}_{2}+$ olefin & cis-( $\left.{ }^{(\mathrm{Bu}} \mathrm{PCP}\right) \mathrm{IrH}_{2}+$ olefin & 22.1 & 13.8 \\
\hline alkene & ${ }^{i} \mathrm{Pr}_{2} \mathrm{CHCH}=\mathrm{CHCH}_{3}$ & & \\
\hline $\mathbf{H}_{2}$ & $\mathrm{H}_{2}+2$ & 0.0 & 0.0 \\
\hline trans-2- $\mathrm{H}_{2}$ & trans-( $\left.{ }^{\mathrm{tBu}} \mathrm{PCP}\right) \mathrm{IrH} \mathrm{H}_{2}$ & -11.4 & 1.3 \\
\hline cis-2- $\mathrm{H}_{2}$ & cis-(tBuPCP) $\mathrm{rrH}_{2}$ & -19.9 & -12.0 \\
\hline
\end{tabular}

aThis TS could only be optimized using a superfine gridsize. (All other calculations for alkane dehydrogenations employed ultrafine gridsize, whereas for all calculations involving amines a superfine integration grid size was used.) 
Table S5. Energies of dehydrogenation of 2-methylbutane by ( $\left.{ }^{\mathrm{tBu} 4} \mathrm{PCP}\right)$ Ir referenced to $\mathrm{T}=323.15 \mathrm{~K}\left(50^{\circ} \mathrm{C}\right)(\mathrm{P}=1 \mathrm{~atm})$.

\begin{tabular}{|c|c|c|c|}
\hline Name & Structure & $\Delta \mathrm{E}$ & $\Delta \mathbf{G}$ \\
\hline 1 & $\mathrm{Me}_{2} \mathrm{CHCH}_{2} \mathrm{CH}_{3}$ & & \\
\hline 2 & (tBuPCP)Ir & & \\
\hline 3 & $1+2$ & 0.0 & 0.0 \\
\hline \multicolumn{4}{|c|}{ pathway a } \\
\hline TS1 & terminal $\mathrm{C}-\mathrm{H}$ activation & 12.2 & 24.9 \\
\hline 5 & terminal-alkyl-(PCP)|r-complex & 3.9 & 16.7 \\
\hline TS2 & $\begin{array}{l}\beta-\mathrm{H} \text { elimination from internal } \\
\text { position }\end{array}$ & 23.2 & 36.6 \\
\hline \multicolumn{4}{|c|}{ pathway b } \\
\hline 'TS1' & internal $\mathrm{C}-\mathrm{H}$ activation & 15.6 & 29.6 \\
\hline $5^{\prime}$ & internal-alkyl-(PCP)Ir complex & 14.6 & 29.9 \\
\hline TS2' & $\begin{array}{l}\beta \text {-H elimination from terminal } \\
\text { position }\end{array}$ & 24.6 & 38.8 \\
\hline 6 & $(\mathrm{PCP}) \operatorname{Ir}(\mathrm{H})_{2}$ (olefin) & 10.6 & 24.4 \\
\hline trans-2- $\mathrm{H}_{2}+$ olefin & trans-( $\left.{ }^{\mathrm{B} u P C P}\right) \mathrm{IrH} \mathrm{H}_{2}+$ olefin & 30.9 & 27.8 \\
\hline cis-2- $\mathrm{H}_{2}+$ olefin & cis-(tBupCP) $\mid \mathrm{rH}_{2}+$ olefin & 22.4 & 14.5 \\
\hline alkene & $\mathrm{Me}_{2} \mathrm{CHCH}=\mathrm{CH}_{2}$ & & \\
\hline
\end{tabular}


Table S6. Energies of dehydrogenation of cyclooctane (COA) by ( $\left.{ }^{\text {tBu4}} \mathrm{PCP}\right)$ Ir referenced to $\mathrm{T}=323.15 \mathrm{~K}\left(50^{\circ} \mathrm{C}\right)(\mathrm{P}=1 \mathrm{~atm})$.

\begin{tabular}{|c|c|c|c|}
\hline Name & Structure & $\Delta \mathrm{E}$ & $\Delta \mathbf{G}$ \\
\hline 1 & COA & & \\
\hline 2 & (tBuPCP) Ir & & \\
\hline 3 & $1+2$ & 0.0 & 0.0 \\
\hline TS1 & $\mathrm{C}-\mathrm{H}$ activation & 15.7 & 29.3 \\
\hline 5 & (PCP)Ir-alkyl complex & 10.7 & 25.5 \\
\hline TS2 & $\beta$-H elimination & 22.7 & 37.0 \\
\hline${ }^{\mathrm{b}} 6$ & $(\mathrm{PCP}) \operatorname{Ir}(\mathrm{H})_{2}$ (olefin) & 14.6 & 30.1 \\
\hline trans $-2-\mathrm{H}_{2}+$ olefin & trans-(tBuPCP) $\mid \mathrm{rH}_{2}+$ olefin & 22.4 & 19.1 \\
\hline cis-2- $\mathrm{H}_{2}+$ olefin & cis-( $\left.{ }^{\mathrm{B} u P C P}\right) \mathrm{IrH} \mathrm{H}_{2}+$ olefin & 13.9 & 5.8 \\
\hline alkene & COE & & \\
\hline
\end{tabular}

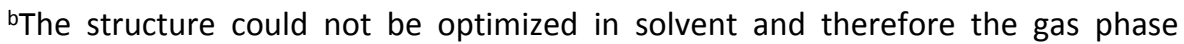
geometry was used for single point energy calculation with the thermal corrections used from gas phase optimization

Table S7. Energies of dehydrogenation of $n$-butane by ( $\left.{ }^{\mathrm{tBu} 4} \mathrm{PCP}\right)$ Ir referenced to $\mathrm{T}=323.15 \mathrm{~K}\left(50^{\circ} \mathrm{C}\right)(\mathrm{P}=1 \mathrm{~atm})$.

\begin{tabular}{|c|c|c|c|}
\hline Name & Structure & $\Delta \mathrm{E}$ & $\Delta \mathbf{G}$ \\
\hline 1 & $\mathrm{CH}_{3} \mathrm{CH}_{2} \mathrm{CH}_{2} \mathrm{CH}_{3}$ & & \\
\hline 2 & (tBuPCP)Ir & & \\
\hline 3 & $1+2$ & 0.0 & 0.0 \\
\hline \multicolumn{4}{|c|}{ pathway a } \\
\hline TS1 & terminal $\mathrm{C}-\mathrm{H}$ activation & 11.7 & 24.1 \\
\hline 5 & terminal-alkyl-(PCP)|r-complex & 4.6 & 18.2 \\
\hline TS2 & $\begin{array}{l}\beta-\mathrm{H} \text { elimination from internal } \\
\text { position }\end{array}$ & 23.5 & 37.3 \\
\hline \multicolumn{4}{|c|}{ pathway b } \\
\hline TS1' & internal $\mathrm{C}-\mathrm{H}$ activation & 17.6 & 31.4 \\
\hline $5^{\prime}$ & internal-alkyl-(PCP)Ir complex & 10.8 & 23.0 \\
\hline TS2' & $\begin{array}{c}\beta \text {-H elimination from terminal } \\
\text { position }\end{array}$ & 25.3 & 39.0 \\
\hline 6 & $(\mathrm{PCP}) \operatorname{Ir}(\mathrm{H})_{2}$ (olefin) & 10.9 & 25.0 \\
\hline trans-2- $\mathrm{H}_{2}+$ olefin & trans-( $\left.{ }^{\mathrm{B} B u} \mathrm{PCP}\right) \mathrm{IrH} \mathrm{H}_{2}+$ olefin & 30.9 & 27.7 \\
\hline cis-2- $\mathrm{H}_{2}+$ olefin & cis-( $\left.{ }^{\text {(BupPCP }}\right) \mathrm{IrH}_{2}+$ olefin & 22.4 & 14.4 \\
\hline alkene & $\mathrm{CH}_{3} \mathrm{CH}_{2} \mathrm{CH}=\mathrm{CH}_{2}$ & & \\
\hline
\end{tabular}




\section{Kinetic Isotope Effect Calculations}

Table S8. Kinetic Isotope Effects for dehydrogenation of $\mathrm{N}$-ethyldiisopropylamine, calculated based on pathways of Scheme S1 and rate-determining $\beta$ - $\mathrm{H}$ elimination by $\left({ }^{\mathrm{tBu} 4} \mathrm{PCP}\right) \mathrm{Ir}$, referenced to $\mathrm{T}=323.15 \mathrm{~K}\left(50^{\circ} \mathrm{C}\right)$.

\begin{tabular}{|c|l|c|c|}
\hline & isotopologue & $\begin{array}{c}\Delta \Delta \mathrm{G} \\
(\mathrm{kcal} / \mathrm{mol})\end{array}$ & KIE \\
\hline \multirow{5}{*}{ pathway a } & ${ }^{i} \mathrm{Pr}_{2} \mathrm{NCH}_{2} \mathrm{CH}_{3}$ & 0 & -- \\
& ${ }^{i} \operatorname{Pr}_{2} \mathrm{NCD}_{2} \mathrm{CH}_{3}$ & 1.1 & $\mathbf{5 . 2}$ \\
& ${ }^{i} \mathrm{Pr}_{2} \mathrm{NCH}_{2} \mathrm{CD}_{3}$ & 0.6 & $\mathbf{2 . 5}$ \\
& ${ }^{i} \operatorname{Pr}_{2} \mathrm{NCD}_{2} \mathrm{CD}_{3}$ & 1.7 & $\mathbf{1 3 . 1}$ \\
\hline \multirow{5}{*}{ pathway b } & ${ }^{i} \mathrm{Pr}_{2} \mathrm{NCH}_{2} \mathrm{CH}_{3}$ & 0.0 & -- \\
& ${ }^{i} \operatorname{Pr}_{2} \mathrm{NCD}_{2} \mathrm{CH}_{3}$ & 0.7 & $\mathbf{2 . 9}$ \\
& ${ }^{i} \operatorname{Pr}_{2} \mathrm{NCH}_{2} \mathrm{CD}_{3}$ & 0.8 & $\mathbf{3 . 5}$ \\
& ${ }^{i} \mathrm{Pr}_{2} \mathrm{NCD}_{2} \mathrm{CD}_{3}$ & 1.5 & $\mathbf{1 0 . 1}$ \\
\hline
\end{tabular}

Table S9. Kinetic Isotope Effects for dehydrogenation of $N$-ethyldiisopropylamine, calculated based on pathways of Scheme S1 and rate-determining $\beta-\mathrm{H}$ elimination by ( $\left.{ }^{\mathrm{tBu} 4} \mathrm{PCP}\right) \mathrm{Ir}$, referenced to $\mathrm{T}=363.15 \mathrm{~K}\left(90^{\circ} \mathrm{C}\right)$.

\begin{tabular}{|c|l|c|c|}
\hline & isotopologue & $\begin{array}{c}\Delta \Delta \mathrm{G} \\
(\mathrm{kcal} / \mathrm{mol})\end{array}$ & KIE \\
\hline \multirow{4}{*}{ pathway a } & ${ }^{i} \mathrm{Pr}_{2} \mathrm{NCH}_{2} \mathrm{CH}_{3}$ & 0 & $\mathbf{1 . 0}$ \\
& ${ }^{i} \mathrm{Pr}_{2} \mathrm{NCD}_{2} \mathrm{CH}_{3}$ & 1.1 & $\mathbf{4 . 3}$ \\
& ${ }^{i} \mathrm{Pr}_{2} \mathrm{NCH}_{2} \mathrm{CD}_{3}$ & 0.6 & $\mathbf{2 . 3}$ \\
& ${ }^{i} \operatorname{Pr}_{2} \mathrm{NCD}_{2} \mathrm{CD}_{3}$ & 1.7 & $\mathbf{9 . 9}$ \\
\hline \multirow{3}{*}{ pathway b } & ${ }^{i} \operatorname{Pr}_{2} \mathrm{NCH}_{2} \mathrm{CH}_{3}$ & 0.0 & $\mathbf{1 . 0}$ \\
& ${ }^{i} \operatorname{Pr}_{2} \mathrm{NCD}_{2} \mathrm{CH}_{3}$ & 0.7 & $\mathbf{2 . 5}$ \\
& ${ }^{i} \operatorname{Pr}_{2} \mathrm{NCH}_{2} \mathrm{CD}_{3}$ & 0.8 & $\mathbf{3 . 1}$ \\
& ${ }^{i} \operatorname{Pr}_{2} \mathrm{NCD}_{2} \mathrm{CD}_{3}$ & 1.5 & $\mathbf{7 . 8}$ \\
\hline
\end{tabular}




\section{References}

S1. $\quad$ Koch, W.; Holthausen, M. C. A Chemist's Guide to Density Functional Theory; Wiley: New York, 2001.

S2. Zhao, C. Y.; Truhlar, D. G. The M06 Suite of Density Functionals for Main Group Thermochemistry, Kinetics, Noncovalent Interactions, Excited States, and Transition Elements: Two New Functionals and Systematic Testing of Four M06 Functionals and Twelve Other Functionals. Theo. Chem. Acc. 2008, 120, 215-241.

S3. Marenich, A.V.; Cramer, C.J.; Truhlar, D.G. Universal Solvation Model Based on Solute Electron Density and on a Continuum Model of the Solvent Defined by the Bulk Dielectric Constant and Atomic Surface Tensions. J. Phys. Chem. B 2009, 113, 6378-6396.

S4. Andrae, D.; Haeussermann, U.; Dolg, M.; Stoll, H.; Preuss, H. Energy-adjusted ab initio pseudopotentials for the second and third row transition elements. Theor. Chim. Acta 1990, 77, 123-41.

S5. (a) Ditchfield, R.; Hehre, W. J.; Pople, J. A. Self-consistent molecular-orbital methods. IX. Extended Gaussiantype basis for molecular-orbital studies of organic molecules. J. Chem. Phys. 1971, 54, 724-728. (b) Hariharan, P. C.; Pople, J. A. Accuracy of AHn equilibrium geometries by single determinant molecular orbital theory. Mol. Phys. 1974, 27, 209-214. (c) Krishnan, R.; Binkley, J. S.; Seeger, R.; Pople, J. A. Self-consistent molecular orbital methods. XX. A basis set for correlated wave functions. J. Chem. Phys. 1980, 72, 650-654.

S6. Hratchian, H. P. ; Schlegel, H. B. in Theory and Applications of Computational Chemistry: The First 40 Years, Dykstra, C. E.; Frenking, G.; Kim, K. S.; Scuseria, G. Ed; Elsevier: Amsterdam, 2005, pp. 195-249.

S7. McQuarrie, D. A. Statistical Thermodynamics; Harper and Row: New York, 1973.

S8. $\quad$ Frisch, $\mathbb{E}$.; Frisch, M. J.; Clemente, F. R.; G. W. Trucks Gaussian 09 User's Reference, 147.

S9. Jansson, C.M.; Salhi-Benachenhou, N.; Lunell, S.; Larsson, P.E.; Bergson, G. Theoretical Calculations of Kinetic Isotope Effects for a Series of Substituted Aziridines. Brändas, E.J.; Kryachko, E.S. (eds) Fundamental World of Quantum Chemistry, Vol. III. Springer: Dordrecht, 2004, pp. 601-617.

S10. Frisch, M. J.; Trucks, G. W.; Schlegel, H. B.; Scuseria, G. E.; Robb, M. A.; Cheeseman, J. R.; Scalmani, G.; V. Barone; Petersson, G. A.; Nakatsuji, H.; X. Li; Caricato, M.; Marenich, A. V.; Bloino, J.; Janesko, B. G.; Gomperts, R.; Mennucci, B.; Hratchian, H. P.; Ortiz, J. V.; Izmaylov, A. F.; Sonnenberg, J. L.; Williams-Young, D.; Ding, F.; Lipparini, F.; Egidi, F.; Goings, J.; Peng, B.; Petrone, A.; Henderson, T.; Ranasinghe, D.; Zakrzewski, V. G.; Gao, J.; Rega, N.; Zheng, G.; Liang, W.; M. Hada; Ehara, M.; Toyota, K.; Fukuda, R.; Hasegawa, J.; Ishida, M.; Nakajima, T.; Honda, Y.; Kitao, O.; Nakai, H.; Vreven, T.; Throssell, K.; Montgomery, J. A.; Peralta, J. E.; Ogliaro, F.; Bearpark, M. J.; Heyd, J. J.; Brothers, E. N.; Kudin, K. N.; Staroverov, V. N.; Keith, T. A.; Kobayashi, R.; Normand, J.; Raghavachari, K.; Rendell, A. P.; J. C. Burant; Iyengar, S. S.; Tomasi, J.; Cossi, M.; Millam, J. M.; Klene, M.; Adamo, C.; Cammi, R.; Ochterski, J. W.; Martin, R. L.; Morokuma, K.; Farkas, O.; Foresman, J. B.; Fox, D. J. Gaussian 16, Revision A.03. Gaussian, Inc., Wallingford, CT, 2016.

S11. GaussView, Version 6; Dennington, R.; Keith, T. A.; Millam, J. M. Semichem Inc., Shawnee Mission, KS, 2016. 


\section{Cartesian Coordinates from DFT Optimizations}

Amines (Optimized with keyword int=superfine)

Common Intermediates

H2

\begin{tabular}{|c|c|c|c|}
\hline \multicolumn{4}{|c|}{ Zero-point correction= } \\
\hline \multicolumn{2}{|c|}{ Thermal correction to Energy= } & \multicolumn{2}{|c|}{0.012686} \\
\hline \multicolumn{4}{|c|}{ Thermal correction to Enthalpy= } \\
\hline \multicolumn{4}{|c|}{ Thermal correction to Gibbs Free Energy $=\quad-0.002610$} \\
\hline \multicolumn{4}{|c|}{ Sum of electronic and zero-point Energies $=\quad-1.158904$} \\
\hline \multicolumn{3}{|c|}{ Sum of electronic and thermal Energies= } & -1.156346 \\
\hline \multicolumn{3}{|c|}{ Sum of electronic and thermal Enthalpies= } & -1.155323 \\
\hline \multicolumn{3}{|c|}{ Sum of electronic and thermal Free Energies= } & -1.171642 \\
\hline \multicolumn{4}{|c|}{ Cartesian Coordinates } \\
\hline \multicolumn{4}{|c|}{ (n) } \\
\hline \multicolumn{4}{|c|}{$1 \quad 0.000000 \quad 0.000000 \quad 0.371184$} \\
\hline \multicolumn{4}{|c|}{$1 \quad 0.000000 \quad 0.000000 \quad-0.371184$} \\
\hline
\end{tabular}

2

The smallest/imaginary frequencies is: $26.2480 \mathrm{~cm}(-1)$

$\begin{array}{lc}\text { Zero-point correction }= & 0.615142 \\ \text { Thermal correction to Energy= } & 0.654449 \\ \text { Thermal correction to Enthalpy= } & 0.655473 \\ \text { Thermal correction to Gibbs Free Energy= } & 0.546103 \\ \text { Sum of electronic and zero-point Energies }= & -1726.214965 \\ \text { Sum of electronic and thermal Energies= } & -1726.175658 \\ \text { Sum of electronic and thermal Enthalpies= } & -1726.174635 \\ \text { Sum of electronic and thermal Free Energies= } & -1726.284005\end{array}$

Cartesian Coordinates

$\begin{array}{ccccccccc}6 & 0.000000 & -0.000000 & -1.556805 & 1 & 1.357398 & -1.663695 & -4.233600 \\ 15 & 1.105870 & -2.022835 & 0.184276 & 1 & 0.000000 & -0.000000 & -5.492276 \\ 15 & -1.105870 & 2.022835 & 0.184276 & 1 & -1.357398 & 1.663695 & -4.233600 \\ 6 & 1.650923 & -1.919508 & -1.581681 & 1 & 2.687504 & -1.552988 & -1.564409 \\ 6 & 2.725401 & -2.299914 & 1.134445 & 1 & 1.678311 & -2.898102 & -2.082778 \\ 6 & 0.000000 & -3.562875 & 0.266445 & 1 & -2.687504 & 1.552988 & -1.564409 \\ 6 & -1.650923 & 1.919508 & -1.581681 & 1 & -1.678311 & 2.898102 & -2.082778 \\ 6 & -2.725401 & 2.299914 & 1.134445 & 6 & -3.671798 & 3.315271 & 0.495225 \\ 6 & -0.000000 & 3.562875 & 0.266445 & 1 & -3.260271 & 4.328815 & 0.473270 \\ 6 & 0.774304 & -0.929843 & -2.302621 & 1 & -3.949389 & 3.040278 & -0.529476 \\ 6 & 0.756160 & -0.927889 & -3.696949 & 1 & -4.602953 & 3.355737 & 1.079586 \\ 6 & 0.000000 & -0.000000 & -4.404251 & 6 & -0.728270 & 4.889953 & 0.081177 \\ 6 & -0.774304 & 0.929843 & -2.302621 & 1 & -1.330298 & 4.913260 & -0.836671 \\ 6 & -0.756160 & 0.927889 & -3.696949 & 1 & -1.380229 & 5.131499 & 0.929265\end{array}$




$\begin{array}{rrrrrrrr}1 & 0.010977 & 5.700966 & 0.003999 & 6 & -1.036203 & -3.406778 & -0.849862 \\ 6 & 3.671798 & -3.315271 & 0.495225 & 1 & -0.590569 & -3.454671 & -1.851062 \\ 1 & 3.260271 & -4.328815 & 0.473270 & 1 & -1.765953 & -4.226051 & -0.774343 \\ 1 & 3.949389 & -3.040278 & -0.529476 & 1 & -1.578774 & -2.455973 & -0.765819 \\ 1 & 4.602953 & -3.355737 & 1.079586 & 6 & -2.423774 & 2.714216 & 2.572826 \\ 6 & 0.728270 & -4.889953 & 0.081177 & 1 & -3.348853 & 2.678680 & 3.166337 \\ 1 & 1.330298 & -4.913260 & -0.836671 & 1 & -1.701096 & 2.038116 & 3.051226 \\ 1 & 1.380229 & -5.131499 & 0.929265 & 1 & -2.034998 & 3.737423 & 2.640966 \\ 1 & -0.010977 & -5.700966 & 0.003999 & 6 & 0.751363 & 3.567328 & 1.598679 \\ 6 & -3.426018 & 0.937887 & 1.169587 & 1 & 0.089993 & 3.706079 & 2.461328 \\ 1 & -3.641764 & 0.547535 & 0.166555 & 1 & 1.307277 & 2.630216 & 1.739178 \\ 1 & -2.817529 & 0.183177 & 1.684536 & 1 & 1.476607 & 4.394349 & 1.605748 \\ 1 & -4.384069 & 1.035830 & 1.701326 & 6 & 2.423774 & -2.714216 & 2.572826 \\ 6 & 1.036203 & 3.406778 & -0.849862 & 1 & 3.348853 & -2.678680 & 3.166337 \\ 1 & 0.590569 & 3.454671 & -1.851062 & 1 & 1.701096 & -2.038116 & 3.051226 \\ 1 & 1.765953 & 4.226051 & -0.774343 & 1 & 2.034998 & -3.737423 & 2.640966 \\ 1 & 1.578774 & 2.455973 & -0.765819 & 6 & -0.751363 & -3.567328 & 1.598679 \\ 6 & 3.426018 & -0.937887 & 1.169587 & 1 & -0.089993 & -3.706079 & 2.461328 \\ 1 & 3.641764 & -0.547535 & 0.166555 & 1 & -1.307277 & -2.630216 & 1.739178 \\ 1 & 2.817529 & -0.183177 & 1.684536 & 1 & -1.476607 & -4.394349 & 1.605748 \\ 1 & 4.384069 & -1.035830 & 1.701326 & 77 & -0.000000 & 0.000000 & 0.427958 \\ -------------------------------------------------------------------------------------------------- & \end{array}$

trans-2-H2

The smallest/imaginary frequencies is: $32.5863 \mathrm{~cm}(-1)$

$\begin{array}{lc}\text { Zero-point correction }= & 0.632720 \\ \text { Thermal correction to Energy= } & 0.671935 \\ \text { Thermal correction to Enthalpy= } & 0.672958 \\ \text { Thermal correction to Gibbs Free Energy= } & 0.564990 \\ \text { Sum of electronic and zero-point Energies }= & -1727.384844 \\ \text { Sum of electronic and thermal Energies }= & -1727.345630 \\ \text { Sum of electronic and thermal Enthalpies }= & -1727.344607 \\ \text { Sum of electronic and thermal Free Energies }= & -1727.452575\end{array}$

Cartesian Coordinates

$\begin{array}{cccccccc}6 & -0.000000 & -1.516382 & -0.000002 & 1 & 0.000003 & -5.425981 & -0.000002 \\ 15 & 2.329392 & 0.202334 & -0.115399 & 1 & -2.028983 & -4.170108 & 0.706653 \\ 15 & -2.329393 & 0.202332 & 0.115399 & 1 & 2.323765 & -1.328044 & -1.948250 \\ 6 & 2.363739 & -1.489866 & -0.860187 & 1 & 3.291174 & -2.039785 & -0.648041 \\ 6 & 3.367033 & 1.259813 & -1.309572 & 1 & -2.323766 & -1.328051 & 1.948247 \\ 6 & 3.236585 & 0.024199 & 1.550226 & 1 & -3.291174 & -2.039789 & 0.648036 \\ 6 & -2.363740 & -1.489869 & 0.860184 & 6 & -4.631928 & 0.552931 & 1.800766 \\ 6 & -3.367031 & 1.259811 & 1.309574 & 1 & -5.336024 & 0.310436 & 1.000941 \\ 6 & -3.236586 & 0.024201 & -1.550227 & 1 & -4.402654 & -0.370836 & 2.345542 \\ 6 & 1.139689 & -2.238747 & -0.410353 & 1 & -5.156188 & 1.216712 & 2.503480 \\ 6 & 1.131049 & -3.633711 & -0.396926 & 6 & -4.737118 & -0.232077 & -1.425934 \\ 6 & 0.000002 & -4.338161 & -0.000002 & 1 & -4.965855 & -1.095350 & -0.787513 \\ 6 & -1.139689 & -2.238749 & 0.410348 & 1 & -5.287972 & 0.637062 & -1.049044 \\ 6 & -1.131046 & -3.633713 & 0.396922 & 1 & -5.140112 & -0.455556 & -2.424332 \\ 1 & 2.028986 & -4.170104 & -0.706657 & 6 & 4.631929 & 0.552932 & -1.800765\end{array}$




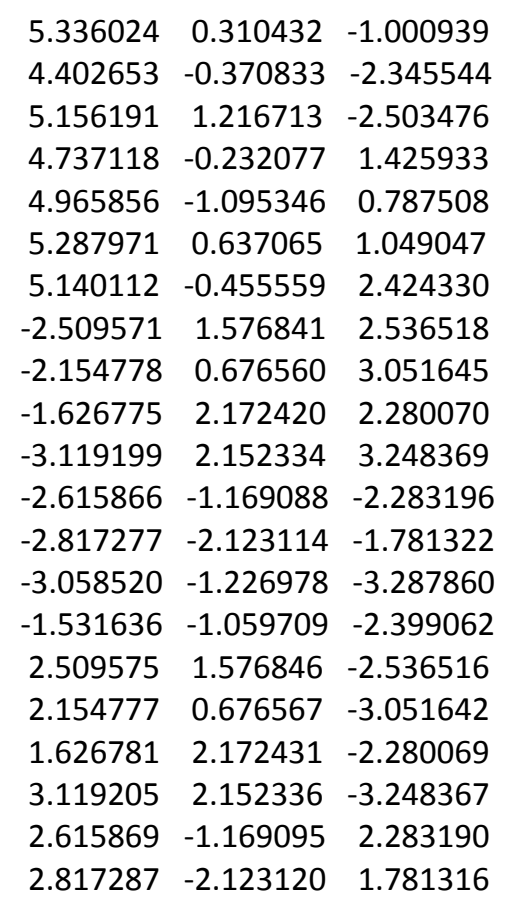

cis-2-H2

The smallest/imaginary frequencies is: $20.4745 \mathrm{~cm}(-1)$

$\begin{array}{lc}\text { Zero-point correction= } & 0.629161 \\ \text { Thermal correction to Energy= } & 0.670005 \\ \text { Thermal correction to Enthalpy= } & 0.671028 \\ \text { Thermal correction to Gibbs Free Energy= } & 0.557984 \\ \text { Sum of electronic and zero-point Energies }= & -1727.401976 \\ \text { Sum of electronic and thermal Energies }= & -1727.361132 \\ \text { Sum of electronic and thermal Enthalpies }= & -1727.360109 \\ \text { Sum of electronic and thermal Free Energies }= & -1727.473152\end{array}$

Cartesian Coordinates

$\begin{array}{crrr}6 & 0.000000 & -1.639823 & -0.000024 \\ 15 & -2.305606 & 0.165735 & 0.067475 \\ 15 & 2.305607 & 0.165735 & -0.067467 \\ 6 & -2.476526 & -1.639493 & 0.498202 \\ 6 & -3.287304 & 1.025982 & 1.441030 \\ 6 & -3.163399 & 0.327168 & -1.616083 \\ 6 & 2.476528 & -1.639480 & -0.498247 \\ 6 & 3.287311 & 1.026021 & -1.440992 \\ 6 & 3.163393 & 0.327119 & 1.616099 \\ 6 & -1.190647 & -2.372782 & 0.211584 \\ 6 & -1.187327 & -3.769531 & 0.202477 \\ 6 & -0.000005 & -4.465014 & -0.000088 \\ 6 & 1.190645 & -2.372776 & -0.211663 \\ 6 & 1.187320 & -3.769526 & -0.202620\end{array}$

$\begin{array}{rrrr}1 & -2.116124 & -4.317193 & 0.367256 \\ 1 & -0.000007 & -5.553725 & -0.000114 \\ 1 & 2.116114 & -4.317184 & -0.367425 \\ 1 & -2.684882 & -1.687067 & 1.576634 \\ 1 & -3.342699 & -2.106583 & 0.006744 \\ 1 & 2.684894 & -1.687023 & -1.576678 \\ 1 & 3.342695 & -2.106586 & -0.006794 \\ 6 & 4.629645 & 0.370307 & -1.766631 \\ 1 & 5.340732 & 0.415676 & -0.936556 \\ 1 & 4.524145 & -0.679354 & -2.066235 \\ 1 & 5.087434 & 0.900690 & -2.614366 \\ 6 & 4.671906 & 0.099877 & 1.581748 \\ 1 & 4.948200 & -0.846689 & 1.099423 \\ 1 & 5.202380 & 0.914922 & 1.075085\end{array}$




$\begin{array}{rrr}5.053075 & 0.061313 & 2.612721 \\ -4.629635 & 0.370258 & 1.766657 \\ -5.340726 & 0.415647 & 0.936586 \\ -4.524133 & -0.679411 & 2.066233 \\ -5.087421 & 0.900618 & 2.614407 \\ -4.671913 & 0.099924 & -1.581732 \\ -4.948203 & -0.846658 & -1.099435 \\ -5.202385 & 0.914952 & -1.075041 \\ -5.053086 & 0.061393 & -2.612704 \\ 2.401198 & 0.961365 & -2.689969 \\ 2.156170 & -0.068703 & -2.981627 \\ 1.454833 & 1.495245 & -2.541885 \\ 2.933328 & 1.424798 & -3.533506 \\ 2.522135 & -0.722634 & 2.528550 \\ 2.729821 & -1.750353 & 2.206017 \\ 2.930761 & -0.607089 & 3.542831 \\ 1.432689 & -0.598645 & 2.584454 \\ -2.401184 & 0.961291 & 2.690000 \\ -2.156152 & -0.068785 & 2.981626 \\ -1.454821 & 1.495178 & 2.541927 \\ -2.933312 & 1.424698 & 3.533554 \\ -2.522144 & -0.722557 & -2.528566\end{array}$

$\begin{array}{lllll}1 & -2.729827 & -1.750286 & -2.206062\end{array}$

$\begin{array}{lllll}1 & -2.930775 & -0.606985 & -3.542843\end{array}$

$\begin{array}{lllll}1 & -1.432699 & -0.598565 & -2.584472\end{array}$

$\begin{array}{lllll}6 & 3.510312 & 2.494085 & -1.084917\end{array}$

$\begin{array}{lllll}1 & 3.891491 & 3.028387 & -1.967132\end{array}$

$\begin{array}{lllll}1 & 2.579414 & 2.986861 & -0.775070\end{array}$

$1 \quad 4.252565 \quad 2.618955 \quad-0.286848$

$\begin{array}{llll}6 & 2.870078 & 1.704224 & 2.212479\end{array}$

$\begin{array}{lllll}1 & 3.298733 & 2.523200 & 1.624373\end{array}$

$\begin{array}{llll}1 & 1.790611 & 1.878375 & 2.298753\end{array}$

$\begin{array}{lllll}1 & 3.306864 & 1.759889 & 3.220314\end{array}$

$\begin{array}{llll}6 & -3.510307 & 2.494055 & 1.084996\end{array}$

$\begin{array}{lllll}1 & -3.891481 & 3.028332 & 1.967229\end{array}$

$\begin{array}{lllll}1 & -2.579411 & 2.986841 & 0.775158\end{array}$

$\begin{array}{lllll}1 & -4.252566 & 2.618948 & 0.286936\end{array}$

$\begin{array}{lllll}6 & -2.870088 & 1.704290 & -2.212424\end{array}$

$\begin{array}{lllll}1 & -3.298745 & 2.523249 & -1.624294\end{array}$

$\begin{array}{lllll}1 & -1.790622 & 1.878447 & -2.298694\end{array}$

$\begin{array}{lllll}1 & -3.306875 & 1.759983 & -3.220257\end{array}$

$\begin{array}{lllll}77 & 0.000001 & 0.479662 & 0.000005\end{array}$

$\begin{array}{lllll}1 & -0.000350 & 1.920080 & -0.729508\end{array}$

$\begin{array}{llll}1 & 0.000351 & 1.920062 & 0.729553\end{array}$

\section{Geometries from ('Pr) ${ }_{2} \mathrm{NCH}_{2} \mathrm{CH}_{3}$ dehydrogenation}

\section{1}

The smallest/imaginary frequencies is: $77.7613 \mathrm{~cm}(-1)$
Zero-point correction=
0.261141
Thermal correction to Energy=
0.274616
Thermal correction to Enthalpy=
0.275639
Thermal correction to Gibbs Free Energy= $\quad 0.221244$
Sum of electronic and zero-point Energies=
$-370.494989$
Sum of electronic and thermal Energies=
$-370.481514$
Sum of electronic and thermal Enthalpies= $-370.480490$
Sum of electronic and thermal Free Energies=
$-370.534885$

Cartesian Coordinates 


$\begin{array}{rrrrrrrrr}6 & -1.446463 & -2.097955 & 0.366930 & 1 & 2.047741 & 0.107575 & -2.032415 \\ 1 & -1.931015 & -1.518896 & 1.163704 & 1 & 2.486559 & 1.139553 & -0.662536 \\ 1 & -0.590735 & -2.622014 & 0.810995 & 1 & 3.282838 & -0.424623 & -0.869090 \\ 1 & -2.160010 & -2.861686 & 0.033682 & 6 & -0.669411 & 0.960846 & 0.341573 \\ 6 & -1.021027 & -1.212560 & -0.799409 & 6 & -2.091655 & 1.312997 & -0.077850 \\ 1 & -0.529868 & -1.833897 & -1.562005 & 6 & 0.170519 & 2.229396 & 0.234340 \\ 1 & -1.911981 & -0.809470 & -1.295306 & 1 & -0.705571 & 0.668215 & 1.412440 \\ 6 & 1.243476 & -0.596395 & -0.150302 & 1 & -2.815168 & 0.513184 & 0.112140 \\ 6 & 1.599707 & -0.611948 & 1.335129 & 1 & -2.429238 & 2.191923 & 0.483207 \\ 6 & 2.324251 & 0.101101 & -0.971994 & 1 & -2.124850 & 1.562861 & -1.147019 \\ 1 & 1.259675 & -1.648735 & -0.473554 & 1 & 1.183486 & 2.114463 & 0.630660 \\ 1 & 0.855447 & -1.153145 & 1.931346 & 1 & 0.250044 & 2.535885 & -0.817334 \\ 1 & 2.565425 & -1.113496 & 1.474924 & 1 & -0.303931 & 3.043211 & 0.796257 \\ 1 & 1.699318 & 0.395224 & 1.757843 & 7 & -0.112079 & -0.114147 & -0.488349\end{array}$

4

The smallest/imaginary frequencies is: $18.6528 \mathrm{~cm}(-1)$

$\begin{array}{lc}\text { Zero-point correction= } & 0.878336 \\ \text { Thermal correction to Energy= } & 0.932616 \\ \text { Thermal correction to Enthalpy= } & 0.933639 \\ \text { Thermal correction to Gibbs Free Energy= } & 0.792514 \\ \text { Sum of electronic and zero-point Energies }= & -2096.725322 \\ \text { Sum of electronic and thermal Energies= } & -2096.671042 \\ \text { Sum of electronic and thermal Enthalpies= } & -2096.670019 \\ \text { Sum of electronic and thermal Free Energies= } & -2096.811144\end{array}$

Cartesian Coordinates

$\begin{array}{lrrr}1 & 0.756038 & 4.962855 & 1.615929 \\ 1 & -0.907672 & 5.213089 & 1.033433 \\ 1 & -0.582114 & 5.050686 & 2.763756 \\ 6 & 0.966178 & 4.645306 & -1.295055 \\ 1 & 0.029070 & 4.830038 & -1.834426 \\ 1 & 0.989065 & 5.302870 & -0.421388 \\ 1 & 1.785208 & 4.961670 & -1.957809 \\ 6 & -1.502224 & -4.634802 & -1.383965 \\ 1 & -1.887762 & -5.192661 & -0.525267 \\ 1 & -2.357055 & -4.307204 & -1.987140 \\ 1 & -0.929900 & -5.343893 & -2.000356 \\ 6 & -2.466091 & -4.120168 & 1.797972 \\ 1 & -3.316240 & -4.259939 & 1.117523 \\ 1 & -1.711372 & -4.881714 & 1.568830 \\ 1 & -2.829773 & -4.325196 & 2.815761 \\ 6 & -1.924567 & 2.852428 & 2.177143 \\ 1 & -2.685511 & 3.376373 & 1.586112 \\ 1 & -2.135301 & 1.776820 & 2.121954 \\ 1 & -2.042814 & 3.172440 & 3.222689 \\ 6 & 1.273937 & 2.373856 & -2.241707 \\ 1 & 0.378168 & 2.466975 & -2.869420 \\ 1 & 2.125599 & 2.750860 & -2.827350\end{array}$




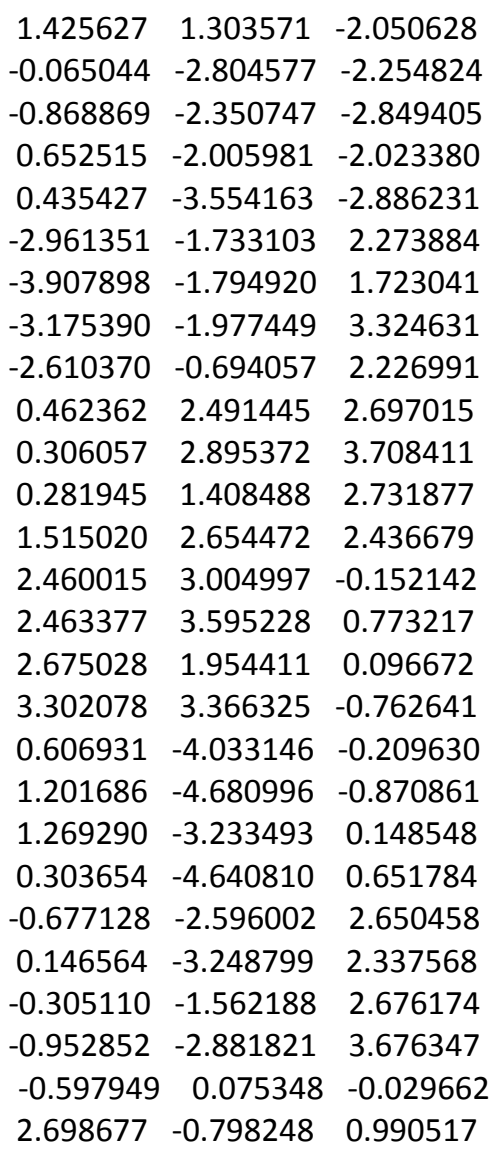

TS1

The smallest/imaginary frequencies is: $-559.9196 \mathrm{~cm}(-1)$

Zero-point correction=

Thermal correction to Energy=

Thermal correction to Enthalpy=

0.873906

0.927647

0.928671

Thermal correction to Gibbs Free Energy=

Sum of electronic and zero-point Energies=

Sum of electronic and thermal Energies=

Sum of electronic and thermal Enthalpies=

Sum of electronic and thermal Free Energies=
0.788313

$-2096.697839$

$-2096.644097$

$-2096.643074$

$-2096.783432$

Cartesian Coordinates

$\begin{array}{cccc}6 & -2.225713 & 0.835455 & -0.921210 \\ 15 & -1.760434 & -1.893118 & 0.001252 \\ 15 & 0.067061 & 2.365839 & 0.091287 \\ 6 & -3.173740 & -1.494406 & -1.131573 \\ 6 & -1.043133 & -3.464824 & -0.795770 \\ 6 & -2.606617 & -2.303436 & 1.652995 \\ 6 & -1.285320 & 3.184975 & -0.883838 \\ 6 & -0.090243 & 3.155919 & 1.814166 \\ 6 & 1.624999 & 2.992818 & -0.811437\end{array}$

$\begin{array}{lrrr}6 & -3.319461 & -0.002588 & -1.258106 \\ 6 & -4.529671 & 0.517102 & -1.719766 \\ 6 & -4.692487 & 1.887805 & -1.884425 \\ 6 & -2.415920 & 2.223613 & -1.132757 \\ 6 & -3.630716 & 2.735655 & -1.595851 \\ 1 & -5.352638 & -0.160434 & -1.952031 \\ 1 & -5.639801 & 2.291547 & -2.237089 \\ 1 & -3.742660 & 3.812522 & -1.731079 \\ 1 & -2.923005 & -1.919135 & -2.114110\end{array}$




\begin{tabular}{lrrr}
1 & -4.107905 & -1.984239 & -0.821454 \\
1 & -1.627336 & 4.111212 & -0.400897 \\
1 & -0.859497 & 3.496817 & -1.847373 \\
6 & 0.230710 & 4.647611 & 1.861634 \\
1 & 1.304265 & 4.843473 & 1.758936 \\
1 & -0.301928 & 5.225681 & 1.095853 \\
1 & -0.074591 & 5.047394 & 2.839754 \\
6 & 1.645444 & 4.496683 & -1.095160 \\
1 & 0.738844 & 4.853684 & -1.598203 \\
1 & 1.791518 & 5.097396 & -0.193390 \\
1 & 2.489616 & 4.715015 & -1.765716 \\
6 & -2.076395 & -4.505265 & -1.231746 \\
1 & -2.582368 & -4.987053 & -0.390639 \\
1 & -2.842628 & -4.089215 & -1.896711 \\
1 & -1.561271 & -5.297864 & -1.794575 \\
6 & -3.419967 & -3.594893 & 1.644631 \\
1 & -4.136696 & -3.639879 & 0.814678 \\
1 & -2.784592 & -4.487402 & 1.603901 \\
1 & -4.000257 & -3.657431 & 2.576907 \\
6 & -1.543670 & 2.950079 & 2.250781 \\
1 & -2.255159 & 3.524969 & 1.645479 \\
1 & -1.832073 & 1.892561 & 2.197186 \\
1 & -1.653314 & 3.284737 & 3.292547 \\
6 & 1.630366 & 2.261981 & -2.158537 \\
1 & 0.774211 & 2.534887 & -2.789073 \\
1 & 2.542179 & 2.529413 & -2.712664 \\
1 & 1.609568 & 1.172955 & -2.037287 \\
6 & -0.308779 & -2.982924 & -2.052198 \\
1 & -0.998198 & -2.575282 & -2.802858 \\
1 & 0.427548 & -2.203424 & -1.828255 \\
1 & 0.215093 & -3.831481 & -2.516489 \\
6 & -3.547732 & -1.136899 & 1.965176 \\
1 & -4.392573 & -1.077666 & 1.268197 \\
1 & -3.962930 & -1.275343 & 2.974000 \\
1 & -3.021974 & -0.174604 & 1.939615 \\
6 & 0.804237 & 2.424801 & 2.815927 \\
1 & 0.692840 & 2.893524 & 3.804599 \\
1 & 0.518018 & 1.370734 & 2.914919 \\
1 & 1.867764 & 2.466817 & 2.552443 \\
6 & 2.894704 & 2.624122 & -0.043648 \\
& & & \\
\hline
\end{tabular}

$\begin{array}{llcc}1 & 3.769768 & 2.908383 & -0.648118 \\ 1 & 2.975059 & 3.172037 & 0.903906 \\ 1 & 2.982692 & 1.548564 & 0.166357 \\ 6 & -0.042796 & -4.128300 & 0.150310 \\ 1 & 0.508603 & -4.911971 & -0.390116 \\ 1 & 0.695386 & -3.419896 & 0.547362 \\ 1 & -0.541258 & -4.612914 & 0.999146 \\ 6 & -1.562283 & -2.376852 & 2.767616 \\ 1 & -0.818711 & -3.165955 & 2.604868 \\ 1 & -1.031595 & -1.423027 & 2.880929 \\ 1 & -2.066791 & -2.593924 & 3.720476 \\ 77 & -0.523077 & 0.091029 & 0.043981 \\ 6 & 2.280805 & -1.117556 & -0.240678 \\ 1 & 1.978199 & -0.553100 & -1.132537 \\ 7 & 3.709902 & -0.871118 & -0.055494 \\ 6 & 4.404147 & -0.641911 & -1.328454 \\ 1 & 3.831815 & 0.179816 & -1.795113 \\ 6 & 4.304357 & -1.693519 & 1.008645 \\ 1 & 3.459714 & -1.912207 & 1.677932 \\ 6 & 4.379563 & -1.795670 & -2.336913 \\ 1 & 4.704480 & -1.433545 & -3.321102 \\ 1 & 5.049745 & -2.615101 & -2.053366 \\ 6 & 5.820775 & -0.118734 & -1.156474 \\ 1 & 6.201783 & 0.226734 & -2.125589 \\ 1 & 5.851157 & 0.725820 & -0.457954 \\ 1 & 6.513073 & -0.891062 & -0.798760 \\ 6 & 4.864989 & -3.050147 & 0.585191 \\ 1 & 5.787164 & -2.953869 & -0.001648 \\ 1 & 5.110559 & -3.645559 & 1.474312 \\ 1 & 4.139091 & -3.623117 & -0.005772 \\ 6 & 5.305655 & -0.926097 & 1.866675 \\ 1 & 4.902894 & 0.061071 & 2.127271 \\ 1 & 5.502346 & -1.474132 & 2.797397 \\ 1 & 6.270369 & -0.778875 & 1.368789 \\ 6 & 1.441693 & -0.697677 & 0.976458 \\ 1 & 0.103788 & -0.208168 & 1.487316 \\ 1 & 1.363018 & -1.545187 & 1.674509 \\ 1 & 2.003387 & 0.079541 & 1.512690 \\ 1 & 3.370992 & -2.210655 & -2.460264 \\ 1 & 2.066618 & -2.178334 & -0.478194 \\ & & & \end{array}$

\section{5}

The smallest/imaginary frequencies is: $20.5907 \mathrm{~cm}(-1)$

$\begin{array}{lc}\text { Zero-point correction }= & 0.874613 \\ \text { Thermal correction to Energy= } & 0.928467 \\ \text { Thermal correction to Enthalpy= } & 0.929491 \\ \text { Thermal correction to Gibbs Free Energy }= & 0.790726 \\ \text { Sum of electronic and zero-point Energies }= & -2096.708974 \\ \text { Sum of electronic and thermal Energies }= & -2096.655119 \\ \text { Sum of electronic and thermal Enthalpies }= & -2096.654095\end{array}$


Cartesian Coordinates

\begin{tabular}{|c|c|c|c|c|c|c|c|}
\hline 6 & -2.309340 & 0.921317 & -0.800483 & 6 & -3.465007 & -1.185746 & 2.128432 \\
\hline 15 & -1.835522 & -1.857053 & -0.010050 & 1 & -4.344055 & -1.067220 & 1.483636 \\
\hline 15 & 0.125483 & 2.342351 & 0.042374 & 1 & -3.828531 & -1.401548 & 3.143263 \\
\hline 6 & -3.327252 & -1.370480 & -1.006105 & 1 & -2.940325 & -0.223534 & 2.158656 \\
\hline 6 & -1.195613 & -3.382983 & -0.938262 & 6 & 1.014238 & 2.311312 & 2.715770 \\
\hline 6 & -2.553086 & -2.332018 & 1.680987 & 1 & 0.991874 & 2.772532 & 3.713903 \\
\hline 6 & -1.263599 & 3.215114 & -0.835820 & 1 & 0.658837 & 1.278410 & 2.811954 \\
\hline 6 & 0.130757 & 3.126778 & 1.771349 & 1 & 2.061977 & 2.275753 & 2.393617 \\
\hline 6 & 1.639446 & 2.878865 & -0.973503 & 6 & 2.939169 & 2.499285 & -0.264044 \\
\hline 6 & -3.457394 & 0.129357 & -1.047833 & 1 & 3.784741 & 2.723304 & -0.932406 \\
\hline 6 & -4.692791 & 0.700767 & -1.357880 & 1 & 3.094221 & 3.087213 & 0.649867 \\
\hline 6 & -4.824617 & 2.081985 & -1.456707 & 1 & 2.999982 & 1.431890 & -0.006418 \\
\hline 6 & -2.468457 & 2.319103 & -0.957747 & 6 & -0.191173 & -4.148380 & -0.078057 \\
\hline 6 & -3.706863 & 2.886886 & -1.266916 & 1 & 0.332546 & -4.888259 & -0.701045 \\
\hline 1 & -5.559723 & 0.058892 & -1.523141 & 1 & 0.566993 & -3.489887 & 0.363157 \\
\hline 1 & -5.790664 & 2.527407 & -1.687191 & 1 & -0.681299 & -4.699951 & 0.733629 \\
\hline 1 & -3.795797 & 3.970426 & -1.361577 & 6 & -1.427254 & -2.476505 & 2.705341 \\
\hline 1 & -3.154166 & -1.745491 & -2.025516 & 1 & -0.734027 & -3.289427 & 2.460365 \\
\hline 1 & -4.241493 & -1.870828 & -0.655544 & 1 & -0.842999 & -1.552844 & 2.798397 \\
\hline 1 & -1.497871 & 4.181655 & -0.367435 & 1 & -1.863946 & -2.702276 & 3.689029 \\
\hline 1 & -0.898509 & 3.458640 & -1.843680 & 77 & -0.519611 & 0.077676 & -0.0144 \\
\hline 6 & 0.597175 & 4.580708 & 1.781055 & 6 & 2.296734 & -1.237213 & -0.28852 \\
\hline 1 & 1.669747 & 4.673046 & 1.574496 & 1 & 2.050108 & -0.761214 & -1.255514 \\
\hline 1 & 0.048936 & 5.208228 & 1.066756 & 7 & 3.709502 & -0.921246 & -0.058601 \\
\hline 1 & 0.425782 & 5.002980 & 2.781869 & 6 & 4.477174 & -0.820838 & -1.302411 \\
\hline 6 & 1.685680 & 4.365637 & -1.332478 & 1 & 3.915349 & -0.078593 & -1.899995 \\
\hline 1 & 0.773742 & 4.718704 & -1.828271 & 6 & 4.257616 & -1.577344 & 1.135292 \\
\hline 1 & 1.870049 & 5.006561 & -0.465921 & 1 & 3.373138 & -1.747385 & 1.765762 \\
\hline 1 & 2.515418 & 4.529779 & -2.035556 & 6 & 4.548531 & -2.084562 & -2.167549 \\
\hline 6 & -2.277920 & -4.347757 & -1.423787 & 1 & 4.932803 & -1.831434 & -3.164557 \\
\hline 1 & -2.827259 & -4.821519 & -0.605079 & 1 & 5.215273 & -2.844414 & -1.744009 \\
\hline 1 & -3.005500 & -3.868185 & -2.088941 & 6 & 5.866817 & -0.234425 & -1.110735 \\
\hline 1 & -1.799171 & -5.152732 & -2.000648 & 1 & 6.300817 & 0.005336 & -2.089620 \\
\hline 6 & -3.378565 & -3.616615 & 1.655381 & 1 & 5.831934 & 0.687740 & -0.518557 \\
\hline 1 & -4.175429 & -3.592742 & 0.901157 & 1 & 6.553840 & -0.935519 & -0.620530 \\
\hline 1 & -2.762643 & -4.506634 & 1.482242 & 6 & 4.899057 & -2.947792 & 0.921982 \\
\hline 1 & -3.863489 & -3.747646 & 2.633613 & 1 & 5.858076 & -2.881118 & 0.392122 \\
\hline 6 & -1.305717 & 3.077887 & 2.300960 & 1 & 5.100821 & -3.421082 & 1.892031 \\
\hline 1 & -1.990228 & 3.720101 & 1.734156 & 1 & 4.240860 & -3.620021 & 0.357360 \\
\hline 1 & -1.717517 & 2.061624 & 2.295604 & 6 & 5.166690 & -0.666615 & 1.955659 \\
\hline 1 & -1.309090 & 3.433885 & 3.341062 & 1 & 4.705176 & 0.322651 & 2.072880 \\
\hline 6 & 1.533337 & 2.079292 & -2.277420 & 1 & 5.323735 & -1.092309 & 2.955619 \\
\hline 1 & 0.627579 & 2.321434 & -2.849233 & 1 & 6.155838 & -0.528931 & 1.504539 \\
\hline 1 & 2.396745 & 2.309006 & -2.918733 & 6 & 1.343181 & -0.756342 & 0.809790 \\
\hline 1 & 1.534972 & 0.996530 & -2.096100 & 1 & -0.951404 & 0.256147 & 1.452051 \\
\hline 6 & -0.472265 & -2.818888 & -2.166676 & 1 & 1.151623 & -1.576114 & 1.521231 \\
\hline 1 & -1.139721 & -2.233568 & -2.813616 & 1 & 1.872627 & 0.013975 & 1.389106 \\
\hline 1 & 0.374392 & -2.180044 & -1.884715 & 1 & 3.560187 & -2.541125 & -2.305246 \\
\hline 1 & -0.075699 & -3.647402 & -2.771334 & 1 & 2.141778 & -2.323400 & -0.4708 \\
\hline
\end{tabular}


TS2

The smallest/imaginary frequencies is: $-780.4563 \mathrm{~cm}(-1)$

$\begin{array}{lc}\text { Zero-point correction= } & 0.871687 \\ \text { Thermal correction to Energy= } & 0.924287 \\ \text { Thermal correction to Enthalpy= } & 0.925310 \\ \text { Thermal correction to Gibbs Free Energy= } & 0.790649 \\ \text { Sum of electronic and zero-point Energies }= & -2096.684526 \\ \text { Sum of electronic and thermal Energies= } & -2096.631926 \\ \text { Sum of electronic and thermal Enthalpies }= & -2096.630903 \\ \text { Sum of electronic and thermal Free Energies= } & -2096.765563\end{array}$

Cartesian Coordinates

\begin{tabular}{|c|c|c|c|c|c|c|c|}
\hline 6 & -0.719438 & -1.913041 & 0.887040 & 1 & -5.299316 & 0.595476 & -2.847070 \\
\hline 15 & -2.469302 & 0.046152 & -0.313253 & 6 & 1.094959 & -3.783634 & -1.300991 \\
\hline 15 & 1.950627 & -1.422456 & -0.045702 & 1 & 1.106244 & -4.353345 & -0.363694 \\
\hline 6 & -3.003425 & -1.708372 & -0.087790 & 1 & 0.109524 & -3.317418 & -1.405048 \\
\hline 6 & -3.293626 & 0.920407 & 1.192589 & 1 & 1.218493 & -4.501277 & -2.125036 \\
\hline 6 & -3.354485 & 0.569861 & -1.920049 & 6 & 3.302833 & 0.568138 & 1.313202 \\
\hline 6 & 1.651026 & -2.361964 & 1.526411 & 1 & 2.837308 & 0.228664 & 2.247916 \\
\hline 6 & 2.233857 & -2.761956 & -1.378692 & 1 & 4.247417 & 1.065157 & 1.584210 \\
\hline 6 & 3.615524 & -0.582616 & 0.357470 & 1 & 2.645255 & 1.312893 & 0.852517 \\
\hline 6 & -2.058258 & -2.363973 & 0.876438 & 6 & -2.327254 & 0.856051 & 2.378004 \\
\hline 6 & -2.481250 & -3.438938 & 1.659043 & 1 & -2.020048 & -0.169696 & 2.611467 \\
\hline 6 & -1.570177 & -4.138931 & 2.444046 & 1 & -1.416748 & 1.437932 & 2.201558 \\
\hline 6 & 0.190950 & -2.688056 & 1.631843 & 1 & -2.833589 & 1.270619 & 3.262436 \\
\hline 6 & -0.229297 & -3.774508 & 2.403553 & 6 & -2.781169 & -0.285581 & -3.054478 \\
\hline 1 & -3.529296 & -3.742410 & 1.633704 & 1 & -2.990460 & -1.354562 & -2.932276 \\
\hline 1 & -1.897325 & -4.976596 & 3.056959 & 1 & -3.247508 & 0.028509 & -3.999158 \\
\hline 1 & 0.507487 & -4.344613 & 2.971850 & 1 & -1.695885 & -0.167041 & -3.149820 \\
\hline 1 & -4.056407 & -1.816317 & 0.195008 & 6 & 2.200568 & -2.106905 & -2.761063 \\
\hline 1 & -2.884437 & -2.181741 & -1.073669 & 1 & 2.316485 & -2.884602 & -3.530064 \\
\hline 1 & 2.283725 & -3.258157 & 1.602233 & 1 & 1.248812 & -1.592274 & -2.936854 \\
\hline 1 & 1.958814 & -1.686684 & 2.338995 & 1 & 3.010253 & -1.381780 & -2.905132 \\
\hline 6 & 3.542502 & -3.536235 & -1.227130 & 6 & 4.246747 & 0.001243 & -0.904983 \\
\hline 1 & 4.427171 & -2.923149 & -1.430466 & 1 & 5.119099 & 0.611259 & -0.626838 \\
\hline 1 & 3.650423 & -3.993241 & -0.235321 & 1 & 4.604990 & -0.772324 & -1.594376 \\
\hline 1 & 3.549998 & -4.356094 & -1.959886 & 1 & 3.549557 & 0.650267 & -1.451540 \\
\hline 6 & 4.629385 & -1.491352 & 1.059076 & 6 & -3.601813 & 2.391346 & 0.913557 \\
\hline 1 & 4.242021 & -1.900331 & 1.999382 & 1 & -3.841917 & 2.889695 & 1.864262 \\
\hline 1 & 4.981535 & -2.322625 & 0.444792 & 1 & -2.753589 & 2.928550 & 0.472847 \\
\hline 1 & 5.510786 & -0.885563 & 1.316116 & 1 & -4.468813 & 2.521372 & 0.255407 \\
\hline 6 & -4.590053 & 0.230402 & 1.626615 & 6 & -3.073666 & 2.034035 & -2.271642 \\
\hline 1 & -5.326558 & 0.133292 & 0.821304 & 1 & -3.604985 & 2.738652 & -1.626963 \\
\hline 1 & -4.402959 & -0.764777 & 2.047241 & 1 & -2.005491 & 2.277134 & -2.234297 \\
\hline 1 & -5.054992 & 0.832015 & 2.421123 & 1 & -3.413580 & 2.222029 & -3.300163 \\
\hline 6 & -4.865835 & 0.351229 & -1.866056 & 77 & -0.112477 & -0.277667 & -0.317002 \\
\hline 1 & -5.131196 & -0.690398 & -1.646088 & 6 & 0.303817 & 2.085959 & -0.374603 \\
\hline 1 & -5.358802 & 0.995992 & -1.129329 & 1 & 0.190422 & 1.024613 & 0.841588 \\
\hline
\end{tabular}




$\begin{array}{rrrrrrrr}7 & 1.303291 & 2.905347 & 0.223724 & 1 & 1.593382 & 5.809890 & 0.157048 \\ 6 & 1.011659 & 3.292460 & 1.613133 & 1 & 1.840517 & 5.793730 & -1.589671 \\ 1 & 0.768237 & 2.331070 & 2.099689 & 1 & 0.308957 & 5.207930 & -0.917505 \\ 6 & 1.988411 & 3.831745 & -0.705425 & 6 & 3.501812 & 3.882709 & -0.528475 \\ 1 & 1.817004 & 3.392134 & -1.694616 & 1 & 3.927703 & 2.873508 & -0.488154 \\ 6 & -0.199853 & 4.207751 & 1.819397 & 1 & 3.949313 & 4.402914 & -1.385231 \\ 1 & -0.533540 & 4.147710 & 2.863607 & 1 & 3.812384 & 4.420686 & 0.373181 \\ 1 & 0.035829 & 5.257686 & 1.610590 & 6 & 0.533844 & 1.394566 & -1.614312 \\ 6 & 2.206585 & 3.857459 & 2.365171 & 1 & -0.417852 & -1.324340 & -1.525685 \\ 1 & 1.945464 & 3.941862 & 3.427293 & 1 & -0.165885 & 1.564622 & -2.432021 \\ 1 & 3.087847 & 3.211980 & 2.288335 & 1 & 1.569640 & 1.323550 & -1.952775 \\ 1 & 2.479183 & 4.864055 & 2.025045 & 1 & -1.053898 & 3.927035 & 1.193396 \\ 6 & 1.393939 & 5.236222 & -0.756452 & 1 & -0.721331 & 2.461255 & -0.251609\end{array}$

TS1'

The smallest/imaginary frequencies is: $-575.0733 \mathrm{~cm}(-1)$

$\begin{array}{lc}\text { Zero-point correction= } & 0.874639 \\ \text { Thermal correction to Energy= } & 0.927761 \\ \text { Thermal correction to Enthalpy= } & 0.928784 \\ \text { Thermal correction to Gibbs Free Energy= } & 0.792720 \\ \text { Sum of electronic and zero-point Energies }= & -2096.685896 \\ \text { Sum of electronic and thermal Energies }= & -2096.632774 \\ \text { Sum of electronic and thermal Enthalpies }= & -2096.631750 \\ \text { Sum of electronic and thermal Free Energies }= & -2096.767814\end{array}$

Cartesian Coordinates

$\begin{array}{cccc}6 & -1.350627 & 2.025927 & 0.127423 \\ 15 & -2.348671 & -0.718012 & -0.148683 \\ 15 & 1.516023 & 1.849512 & 0.072137 \\ 6 & -3.329801 & 0.744675 & -0.728446 \\ 6 & -2.628949 & -1.962674 & -1.568432 \\ 6 & -3.312983 & -1.285398 & 1.396283 \\ 6 & 0.685089 & 3.394748 & 0.680381 \\ 6 & 3.047703 & 1.794569 & 1.205312 \\ 6 & 2.000039 & 2.281997 & -1.722295 \\ 6 & -2.723898 & 2.016671 & -0.213186 \\ 6 & -3.503068 & 3.173627 & -0.167155 \\ 6 & -2.931708 & 4.392132 & 0.183395 \\ 6 & -0.792413 & 3.289433 & 0.430322 \\ 6 & -1.571765 & 4.448958 & 0.461210 \\ 1 & -4.561137 & 3.122153 & -0.427798 \\ 1 & -3.539100 & 5.294727 & 0.218545 \\ 1 & -1.104499 & 5.404600 & 0.703330 \\ 1 & -3.243335 & 0.741205 & -1.825180 \\ 1 & -4.402477 & 0.658308 & -0.504682 \\ 1 & 0.889901 & 3.463374 & 1.757455 \\ 1 & 1.111290 & 4.302625 & 0.228289 \\ 6 & 3.909631 & 3.058588 & 1.235405\end{array}$

$\begin{array}{llll}1 & 4.520389 & 3.187778 & 0.338527 \\ 1 & 3.320260 & 3.971867 & 1.386274 \\ 1 & 4.603737 & 2.981816 & 2.085782 \\ 6 & 3.097489 & 3.335453 & -1.852177 \\ 1 & 2.901946 & 4.237570 & -1.259158 \\ 1 & 4.082487 & 2.943823 & -1.575896 \\ 1 & 3.164024 & 3.648737 & -2.904275 \\ 6 & -4.033048 & -1.900279 & -2.176538 \\ 1 & -4.827561 & -2.100186 & -1.451591 \\ 1 & -4.243390 & -0.937030 & -2.654648 \\ 1 & -4.108829 & -2.667838 & -2.960668 \\ 6 & -4.751942 & -1.718166 & 1.123742 \\ 1 & -5.331791 & -0.959809 & 0.583306 \\ 1 & -4.808010 & -2.660484 & 0.566347 \\ 1 & -5.260244 & -1.884997 & 2.084914 \\ 6 & 2.500382 & 1.607158 & 2.629901 \\ 1 & 2.224332 & 2.566110 & 3.085002 \\ 1 & 1.620542 & 0.957617 & 2.673140 \\ 1 & 3.280876 & 1.165548 & 3.265923 \\ 6 & 0.747893 & 2.805085 & -2.432067 \\ 1 & 0.397972 & 3.762693 & -2.027160 \\ 1 & 0.991376 & 2.964769 & -3.492787\end{array}$




\begin{tabular}{|c|c|c|c|c|c|c|c|}
\hline 1 & -0.081567 & 2.089655 & -2.374840 & 1 & 0.005557 & -2.497430 & 0.534832 \\
\hline 6 & -1.625492 & -1.605704 & -2.668643 & 7 & 2.098679 & -2.294246 & 0.212452 \\
\hline 1 & -1.708598 & -0.558551 & -2.989888 & 6 & 2.149585 & -2.512568 & -1.242013 \\
\hline 1 & -0.591001 & -1.766214 & -2.345247 & 1 & 1.554505 & -1.684373 & -1.65181 \\
\hline 1 & -1.812790 & -2.241388 & -3.546820 & 6 & 2.654093 & -3.352605 & 1.08775 \\
\hline 6 & -3.339322 & -0.080761 & 2.341779 & 1 & 2.745977 & -2.880271 & 2.07356 \\
\hline 1 & -3.912786 & 0.761576 & 1.935930 & 6 & 1.509986 & -3.792973 & -1.78315 \\
\hline 1 & -3.814442 & -0.379635 & 3.287418 & 1 & 1.387088 & -3.712968 & -2.8720 \\
\hline 1 & -2.330276 & 0.285058 & 2.570568 & 1 & 2.133380 & -4.676647 & -1.5966 \\
\hline 6 & 3.919290 & 0.600694 & 0.819097 & 6 & 3.532902 & -2.321465 & -1.8658 \\
\hline 1 & 4.714603 & 0.467952 & 1.568074 & 1 & 3.416085 & -2.082382 & -2.93161 \\
\hline 1 & 3.347960 & -0.336770 & 0.748585 & 1 & 4.069975 & -1.491944 & -1.39165 \\
\hline 1 & 4.416307 & 0.765321 & -0.147043 & 1 & 4.164779 & -3.213728 & $-1.8104 C$ \\
\hline 6 & 2.446992 & 1.012824 & -2.444439 & 6 & 1.778035 & -4.596643 & 1.28870 \\
\hline 1 & 2.743344 & 1.264712 & -3.473563 & 1 & 1.814074 & -5.277024 & 0.43047 \\
\hline 1 & 3.306207 & 0.532124 & -1.961754 & 1 & 2.134333 & -5.157788 & 2.16267 \\
\hline 1 & 1.630082 & 0.282265 & -2.496079 & 1 & 0.724739 & -4.345243 & 1.46989 \\
\hline 6 & -2.363845 & -3.397846 & -1.114362 & 6 & 4.072826 & -3.779528 & 0.741545 \\
\hline 1 & -2.297527 & -4.050132 & -1.997749 & 1 & 4.731554 & -2.916418 & 0.591034 \\
\hline 1 & -1.421405 & -3.499032 & -0.561480 & 1 & 4.475002 & -4.365647 & 1.57768 \\
\hline 1 & -3.172096 & -3.789462 & -0.485084 & 1 & 4.124498 & -4.420949 & -0.14574 \\
\hline 6 & -2.586048 & -2.427095 & 2.108187 & 6 & 0.886710 & -1.521378 & 2.22599 \\
\hline 1 & -2.548086 & -3.347049 & 1.515089 & 1 & 0.800746 & -2.447573 & 2.81847 \\
\hline 1 & -1.561531 & -2.154118 & 2.379768 & 1 & 1.833649 & -1.043480 & 2.49880 \\
\hline 1 & -3.121369 & -2.662855 & 3.039631 & 1 & 0.518636 & -3.978711 & -1.35316 \\
\hline 77 & -0.212457 & 0.251984 & 0.153830 & 1 & 0.549280 & -0.860228 & -0.69022 \\
\hline 6 & 0.811181 & -1.766684 & 0.722432 & 1 & 0.080992 & -0.855837 & 2.562985 \\
\hline
\end{tabular}

$5^{\prime}$

The smallest/imaginary frequencies is: $19.0832 \mathrm{~cm}(-1)$

Zero-point correction=

Thermal correction to Energy=

Thermal correction to Enthalpy=

0.876492

0.929702

0.930726

Thermal correction to Gibbs Free Energy=

Sum of electronic and zero-point Energies=

Sum of electronic and thermal Energies=

Sum of electronic and thermal Enthalpies=

Sum of electronic and thermal Free Energies=
0.795013

$-2096.687730$

$-2096.634520$

$-2096.633497$

$-2096.769209$

Cartesian Coordinates

$\begin{array}{cccc}6 & -0.425393 & -2.271141 & -0.429332 \\ 15 & 2.176748 & -1.081486 & -0.047856 \\ 15 & -2.426392 & -0.281966 & 0.179499 \\ 6 & 1.937613 & -2.513911 & -1.201244 \\ 6 & 3.545520 & -0.117295 & -0.956697 \\ 6 & 2.860704 & -1.857483 & 1.545847 \\ 6 & -2.751941 & -2.084921 & 0.459583 \\ 6 & -3.515582 & 0.473620 & 1.556383 \\ 6 & -3.241075 & 0.012134 & -1.526913\end{array}$

$\begin{array}{lrrr}6 & 0.578360 & -3.102860 & -0.972738 \\ 6 & 0.351411 & -4.447759 & -1.273029 \\ 6 & -0.891494 & -5.022072 & -1.032535 \\ 6 & -1.664028 & -2.897542 & -0.172952 \\ 6 & -1.897005 & -4.240953 & -0.474535 \\ 1 & 1.158295 & -5.049769 & -1.693886 \\ 1 & -1.071412 & -6.069365 & -1.267798 \\ 1 & -2.873787 & -4.679011 & -0.262714 \\ 1 & 2.007368 & -2.095816 & -2.216551\end{array}$ 


$\begin{array}{rrr}2.741776 & -3.258644 & -1.116928 \\ -2.746191 & -2.233417 & 1.549833 \\ -3.752885 & -2.377427 & 0.110935 \\ -4.879128 & -0.201325 & 1.735986 \\ -5.545952 & -0.079432 & 0.879526 \\ -4.795170 & -1.271518 & 1.955897 \\ -5.378436 & 0.261879 & 2.599697 \\ -4.760865 & -0.127926 & -1.539944 \\ -5.104715 & -1.090307 & -1.141296 \\ -5.262080 & 0.678067 & -0.992672 \\ -5.108276 & -0.069055 & -2.581803 \\ 4.553080 & -1.013957 & -1.683302 \\ 5.095626 & -1.686118 & -1.010551 \\ 4.093562 & -1.617992 & -2.472783 \\ 5.300181 & -0.369483 & -2.169554 \\ 4.237593 & -2.496445 & 1.370826 \\ 4.247033 & -3.265854 & 0.588623 \\ 5.023534 & -1.766506 & 1.149441 \\ 4.517780 & -2.993058 & 2.311014 \\ -2.748559 & 0.269658 & 2.866257 \\ -2.611827 & -0.793482 & 3.103330 \\ -1.763244 & 0.738649 & 2.852971 \\ -3.324999 & 0.712829 & 3.691016 \\ -2.667706 & -0.999178 & -2.524310 \\ -2.937250 & -2.034713 & -2.287990 \\ -3.073035 & -0.768195 & -3.520253 \\ -1.573832 & -0.948951 & -2.587971 \\ 2.805860 & 0.714479 & -2.012519 \\ 2.287518 & 0.072148 & -2.739316 \\ 2.060038 & 1.392976 & -1.577175 \\ 3.532914 & 1.316186 & -2.578908 \\ 1.892349 & -2.952298 & 2.003371 \\ 1.820810 & -3.779909 & 1.287646 \\ 2.260683 & -3.364967 & 2.953679 \\ 0.879980 & -2.568508 & 2.172297 \\ -3.738481 & 1.966592 & 1.321471 \\ -4.191571 & 2.414051 & 2.217934 \\ -2.806490 & 2.508030 & 1.119856 \\ -4.426119 & 2.152734 & 0.486845 \\ -2.862922 & 1.400764 & -2.024693\end{array}$

$\begin{array}{cccc}1 & -3.223760 & 2.203957 & -1.370598 \\ 1 & -1.775174 & 1.497442 & -2.115720 \\ 1 & -3.295574 & 1.566020 & -3.022640 \\ 6 & 4.335603 & 0.795853 & -0.017511 \\ 1 & 4.926219 & 1.504417 & -0.616927 \\ 1 & 3.703812 & 1.382972 & 0.656361 \\ 1 & 5.045668 & 0.229822 & 0.597050 \\ 6 & 2.925472 & -0.793507 & 2.642504 \\ 1 & 3.650281 & -0.001449 & 2.425447 \\ 1 & 1.946953 & -0.325388 & 2.806465 \\ 1 & 3.232311 & -1.268434 & 3.585527 \\ 77 & -0.027918 & -0.227176 & 0.134039 \\ 6 & 0.675596 & 1.814645 & 0.827702 \\ 1 & 1.727516 & 1.619484 & 1.081405 \\ 7 & 0.769514 & 2.871184 & -0.221621 \\ 6 & 2.018060 & 3.648586 & -0.206655 \\ 1 & 2.813355 & 2.891249 & -0.259950 \\ 6 & -0.492297 & 3.511994 & -0.586123 \\ 1 & -1.237704 & 2.741732 & -0.337046 \\ 6 & 2.323306 & 4.464656 & 1.056031 \\ 1 & 3.300493 & 4.955649 & 0.948267 \\ 1 & 1.579298 & 5.249181 & 1.240482 \\ 6 & 2.239935 & 4.523109 & -1.435253 \\ 1 & 3.283059 & 4.864306 & -1.441122 \\ 1 & 2.064471 & 3.973991 & -2.367142 \\ 1 & 1.613893 & 5.423945 & -1.437785 \\ 6 & -0.890401 & 4.777853 & 0.182064 \\ 1 & -0.251619 & 5.632213 & -0.078402 \\ 1 & -1.920434 & 5.059744 & -0.082326 \\ 1 & -0.849859 & 4.643005 & 1.267718 \\ 6 & -0.648525 & 3.788221 & -2.085202 \\ 1 & -0.134611 & 4.701124 & -2.402389 \\ 1 & -0.258589 & 2.957161 & -2.686849 \\ 1 & -1.709244 & 3.924633 & -2.335322 \\ 6 & 0.058434 & 2.303180 & 2.138690 \\ 1 & 0.190680 & 1.532802 & 2.912942 \\ 1 & 0.509745 & 3.229454 & 2.530263 \\ 1 & -1.019627 & 2.490521 & 2.064778 \\ 1 & -0.043607 & -0.628235 & 1.626314 \\ 1 & 2.378988 & 3.830052 & 1.947128\end{array}$

\section{TS2'}

The smallest/imaginary frequencies is: $-600.3037 \mathrm{~cm}(-1)$

$\begin{array}{lc}\text { Zero-point correction= } & 0.872900 \\ \text { Thermal correction to Energy= } & 0.925174 \\ \text { Thermal correction to Enthalpy= } & 0.926197 \\ \text { Thermal correction to Gibbs Free Energy= } & 0.792636 \\ \text { Sum of electronic and zero-point Energies }= & -2096.683399\end{array}$


Sum of electronic and thermal Energies= Sum of electronic and thermal Enthalpies= Sum of electronic and thermal Free Energies=
$-2096.631124$

$-2096.630101$

$-2096.763662$

Cartesian Coordinates

$\begin{array}{cccc}\cdots & 0.812188 & -2.121975 & -0.168255 \\ 15 & 2.468035 & 0.216327 & 0.213893 \\ 15 & -1.926497 & -1.341395 & -0.111322 \\ 6 & 2.884525 & -1.408710 & 0.994893 \\ 6 & 3.600338 & 0.252131 & -1.338847 \\ 6 & 3.135708 & 1.462743 & 1.508767 \\ 6 & -1.388247 & -2.829726 & -1.084843 \\ 6 & -2.401586 & -2.046032 & 1.608058 \\ 6 & -3.539305 & -0.865769 & -1.031438 \\ 6 & 2.090153 & -2.476191 & 0.305622 \\ 6 & 2.578912 & -3.778432 & 0.190969 \\ 6 & 1.793561 & -4.775046 & -0.380776 \\ 6 & 0.023785 & -3.156628 & -0.701370 \\ 6 & 0.509112 & -4.461883 & -0.812138 \\ 1 & 3.577657 & -4.013842 & 0.562133 \\ 1 & 2.174124 & -5.790299 & -0.474473 \\ 1 & -0.130114 & -5.239876 & -1.232219 \\ 1 & 3.960949 & -1.614934 & 1.023540 \\ 1 & 2.541065 & -1.329020 & 2.036182 \\ 1 & -2.062460 & -3.688988 & -0.964843 \\ 1 & -1.447231 & -2.518257 & -2.140028 \\ 6 & -3.595074 & -3.001827 & 1.551645 \\ 1 & -4.533215 & -2.512547 & 1.271456 \\ 1 & -3.423922 & -3.843842 & 0.869177 \\ 1 & -3.746608 & -3.430389 & 2.552704 \\ 6 & -4.285011 & -2.073183 & -1.606077 \\ 1 & -3.710940 & -2.584318 & -2.387041 \\ 1 & -4.571105 & -2.813097 & -0.852846 \\ 1 & -5.212197 & -1.713190 & -2.075616 \\ 6 & 4.951792 & -0.432626 & -1.113131 \\ 1 & 5.524163 & -0.026795 & -0.274761 \\ 1 & 4.836985 & -1.513188 & -0.963268 \\ 1 & 5.563200 & -0.300285 & -2.017453 \\ 6 & 4.650626 & 1.359189 & 1.697694 \\ 1 & 4.982941 & 0.335455 & 1.908922 \\ 1 & 5.212715 & 1.738933 & 0.836997 \\ 1 & 4.936728 & 1.974657 & 2.563007 \\ 6 & -1.226006 & -2.835509 & 2.194012 \\ 1 & -0.984867 & -3.732972 & 1.612905 \\ 1 & -0.319425 & -2.226513 & 2.274962 \\ 1 & -1.508196 & -3.161218 & 3.205815 \\ 6 & -3.134458 & 0.038937 & -2.194688 \\ 1 & -2.358077 & -0.407089 & -2.830600 \\ 1 & -4.014509 & 0.221235 & -2.828981 \\ 1 & -2.779526 & 1.006101 & -1.819438 \\ 6 & 2.912241 & -0.515907 & -2.472372 \\ 1 & 2.661059 & -1.544329 & -2.188112 \\ & & & \end{array}$

\begin{tabular}{|c|c|c|c|}
\hline 1 & 1.994201 & -0.029939 & -2.814518 \\
\hline 1 & 3.604577 & -0.562179 & -3.325674 \\
\hline 6 & 2.476694 & 1.152105 & 2.857233 \\
\hline 1 & 2.875941 & 0.243373 & 3.322437 \\
\hline 1 & 2.685910 & 1.982975 & 3.546200 \\
\hline 1 & 1.388394 & 1.046528 & 2.773462 \\
\hline 6 & -2.709127 & -0.905166 & 2.581130 \\
\hline 1 & -2.933038 & -1.334357 & 3.568495 \\
\hline 1 & -1.845689 & -0.237492 & 2.695824 \\
\hline 1 & -3.575595 & -0.304440 & 2.284878 \\
\hline 6 & -4.490299 & -0.062006 & -0.148292 \\
\hline 1 & -5.278673 & 0.373784 & -0.779720 \\
\hline 1 & -4.989763 & -0.669886 & 0.613564 \\
\hline 1 & -3.976286 & 0.766774 & 0.347327 \\
\hline 6 & 3.834590 & 1.687556 & -1.812080 \\
\hline 1 & 4.325698 & 1.663425 & -2.795632 \\
\hline 1 & 2.898940 & 2.250976 & -1.931000 \\
\hline 1 & 4.489387 & 2.254114 & -1.139875 \\
\hline 6 & 2.781733 & 2.912775 & 1.162820 \\
\hline 1 & 3.027318 & 3.203326 & 0.136377 \\
\hline 1 & 1.718909 & 3.114301 & 1.329519 \\
\hline 1 & 3.342229 & 3.579714 & 1.833744 \\
\hline 77 & 0.127529 & -0.113080 & -0.130251 \\
\hline 6 & -0.092116 & 1.473386 & -1.726943 \\
\hline 1 & 0.111854 & -0.104855 & -1.837677 \\
\hline 6 & -0.348659 & 2.057523 & -0.434265 \\
\hline 1 & 0.088289 & -0.196998 & 1.513695 \\
\hline$\perp$ & 0.505569 & 2.601774 & -0.027585 \\
\hline 1 & 0.850536 & 1.707664 & -2.227122 \\
\hline 1 & -0.923951 & 1.427425 & -2.427306 \\
\hline 7 & -1.616464 & 2.709919 & -0.206224 \\
\hline 6 & -2.126079 & 2.574866 & 1.164842 \\
\hline 1 & -2.144930 & 1.487649 & 1.325700 \\
\hline 6 & -1.672765 & 4.081067 & -0.768039 \\
\hline 1 & -2.448833 & 4.614214 & -0.201771 \\
\hline 6 & -2.132014 & 4.057178 & -2.220048 \\
\hline 1 & -2.280267 & 5.077579 & -2.597652 \\
\hline 1 & -1.387718 & 3.576238 & -2.868542 \\
\hline 1 & -3.078479 & 3.512077 & -2.322002 \\
\hline 6 & -0.393474 & 4.911705 & -0.647338 \\
\hline 1 & 0.410218 & 4.522465 & -1.287990 \\
\hline 1 & -0.593837 & 5.941619 & -0.968907 \\
\hline 1 & -0.014139 & 4.952852 & 0.380570 \\
\hline 6 & -1.248086 & 3.164097 & 2.266035 \\
\hline 1 & -1.189416 & 4.259806 & 2.199555 \\
\hline 1 & -1.666569 & 2.919737 & 3.252460 \\
\hline 1 & -0.231569 & 2.753954 & 2.231511 \\
\hline
\end{tabular}




$\begin{array}{llllllll}6 & -3.550676 & 3.101924 & 1.308931 & 1 & -4.153477 & 2.886057 & 0.417328 \\ 1 & -3.582594 & 4.185920 & 1.482132 & 1 & -4.037754 & 2.632788 & 2.174117\end{array}$

6

The smallest/imaginary frequencies is: $21.8350 \mathrm{~cm}(-1)$

$\begin{array}{lc}\text { Zero-point correction }= & 0.873752 \\ \text { Thermal correction to Energy= } & 0.926717 \\ \text { Thermal correction to Enthalpy= } & 0.927741 \\ \text { Thermal correction to Gibbs Free Energy= } & 0.791871 \\ \text { Sum of electronic and zero-point Energies }= & -2096.701793 \\ \text { Sum of electronic and thermal Energies= } & -2096.648827 \\ \text { Sum of electronic and thermal Enthalpies }= & -2096.647804 \\ \text { Sum of electronic and thermal Free Energies }= & -2096.783674\end{array}$

Cartesian Coordinates

\begin{tabular}{|c|c|c|c|c|c|c|c|}
\hline 6 & 1.145286 & -1.929515 & 0.255102 & 1 & 4.776272 & 1.549183 & 1.840102 \\
\hline 15 & 2.462666 & 0.606404 & 0.088985 & 1 & 5.018188 & 2.522381 & 0.368411 \\
\hline 15 & -1.645147 & -1.629061 & -0.196297 & 1 & 4.539853 & 3.298972 & 1.877726 \\
\hline 6 & 2.967022 & -0.646001 & 1.354240 & 6 & -1.181989 & -2.695378 & 2.377507 \\
\hline 6 & 3.693864 & 0.285083 & -1.355353 & 1 & -0.777209 & -3.640581 & 1.998698 \\
\hline 6 & 2.907169 & 2.269810 & 0.931556 & 1 & -0.358170 & -1.978640 & 2.46681 \\
\hline 6 & -0.763037 & -3.157147 & -0.766808 & 1 & -1.575781 & -2.885786 & 3.3865 \\
\hline 6 & -2.328271 & -2.148945 & 1.519972 & 6 & -2.534710 & -0.922338 & -2.7329 \\
\hline 6 & -3.086198 & -1.519980 & -1.437812 & 1 & -1.692761 & -1.492423 & -3.1433 \\
\hline 6 & 2.393927 & -1.956439 & 0.905707 & 1 & -3.335150 & -0.919011 & $-3.4871 \mathrm{~S}$ \\
\hline 6 & 3.078697 & -3.154627 & 1.114213 & 1 & -2.203206 & 0.108414 & -2.593567 \\
\hline 6 & 2.532859 & -4.359945 & 0.683944 & 6 & 3.170215 & -0.843892 & -2.247423 \\
\hline 6 & 0.602683 & -3.161204 & -0.148280 & 1 & 3.008393 & -1.777675 & -1.697079 \\
\hline 6 & 1.290972 & -4.358476 & 0.058574 & 1 & 2.228090 & -0.576914 & -2.735958 \\
\hline 1 & 4.047605 & -3.139736 & 1.615338 & 1 & 3.921662 & -1.037516 & -3.026920 \\
\hline 1 & 3.068765 & -5.293839 & 0.841582 & 6 & 2.137294 & 2.325985 & 2.257382 \\
\hline 1 & 0.842495 & -5.298034 & -0.267143 & 1 & 2.561831 & 1.647911 & 3.007013 \\
\hline 1 & 4.048045 & -0.687264 & 1.532827 & 1 & 2.211211 & 3.343717 & 2.666689 \\
\hline 1 & 2.481905 & -0.332936 & 2.289501 & 1 & 1.076663 & 2.072142 & 2.148986 \\
\hline 1 & -1.322439 & -4.077268 & -0.547292 & 6 & -2.892965 & -0.933684 & 2.2479 \\
\hline 1 & -0.692914 & -3.071441 & -1.861924 & 1 & -3.252500 & -1.243267 & 3.240094 \\
\hline 6 & -3.406976 & -3.229874 & 1.451767 & 1 & -2.114922 & -0.176677 & 2.3917 \\
\hline 1 & -4.343885 & -2.868051 & 1.014128 & 1 & -3.735167 & -0.474328 & 1.72179 \\
\hline 1 & -3.083469 & -4.123445 & 0.903603 & 6 & -4.159292 & -0.576846 & -0.8985 \\
\hline 1 & -3.639739 & -3.551660 & 2.477128 & 1 & -4.866626 & -0.330627 & -1.7048 \\
\hline 6 & -3.704393 & -2.872453 & -1.801616 & 1 & -4.741100 & -1.026672 & -0.0848 \\
\hline 1 & -2.982067 & -3.539256 & -2.287358 & 1 & -3.714227 & 0.362108 & -0.5392 \\
\hline 1 & -4.141999 & -3.403885 & -0.953951 & 6 & 3.835666 & 1.526900 & -2.2355 \\
\hline 1 & -4.513925 & -2.697075 & -2.525257 & 1 & 4.386135 & 1.253675 & -3.147140 \\
\hline 6 & 5.076327 & -0.145955 & -0.857315 & 1 & 2.862564 & 1.923853 & $-2.5546 \mathrm{~S}$ \\
\hline 1 & 5.536072 & 0.555236 & -0.154869 & 1 & 4.401330 & 2.330653 & -1.751325 \\
\hline 1 & 5.045432 & -1.136142 & -0.386992 & 6 & 2.503053 & 3.483123 & 0.08584 \\
\hline 1 & 5.749111 & -0.224984 & -1.723436 & 1 & 3.077875 & 3.568021 & -0.84007 \\
\hline 6 & 4.395207 & 2.399458 & 1.261306 & 1 & 1.440906 & 3.493219 & -0.179267 \\
\hline
\end{tabular}




$\begin{array}{cccccccc}1 & 2.699460 & 4.394287 & 0.669405 & 1 & -4.018170 & 3.731365 & -2.400228 \\ 77 & 0.187875 & -0.110377 & -0.186452 & 1 & -2.938601 & 2.339550 & -2.570900 \\ 6 & -0.320019 & 1.675632 & -1.442327 & 1 & -4.340608 & 2.244479 & -1.487409 \\ 1 & 0.392708 & -0.627792 & -1.759258 & 6 & -1.730807 & 4.505962 & -1.203630 \\ 6 & -0.753104 & 2.053060 & -0.169687 & 1 & -1.073383 & 4.124038 & -1.993911 \\ 1 & 0.039551 & 0.127077 & 1.483831 & 1 & -2.292683 & 5.348384 & -1.625768 \\ 1 & -0.031660 & 2.544972 & 0.482263 & 1 & -1.097996 & 4.897611 & -0.398313 \\ 1 & 0.632116 & 2.042118 & -1.822590 & 6 & -1.748220 & 3.829198 & 2.162997 \\ 1 & -1.053481 & 1.487995 & -2.220891 & 1 & -2.274756 & 4.719777 & 1.794155 \\ 7 & -2.108179 & 2.362782 & 0.127103 & 1 & -1.855783 & 3.820268 & 3.254994 \\ 6 & -2.327864 & 2.541944 & 1.574473 & 1 & -0.679394 & 3.951019 & 1.943536 \\ 1 & -1.789264 & 1.700599 & 2.032239 & 6 & -3.799743 & 2.417529 & 1.942620 \\ 6 & -2.709292 & 3.445823 & -0.703271 & 1 & -4.367861 & 3.326513 & 1.703474 \\ 1 & -3.408958 & 3.964397 & -0.033206 & 1 & -4.271954 & 1.578894 & 1.418190 \\ 6 & -3.546262 & 2.904770 & -1.852719 & 1 & -3.906959 & 2.250016 & 3.021760 \\ ---------------------------------------------------------------------------------------------------------- & \end{array}$

enamine

The smallest/imaginary frequencies is: $26.8212 \mathrm{~cm}(-1)$

\begin{abstract}
Zero-point correction=
Thermal correction to Energy=

Thermal correction to Enthalpy=

0.237940

0.251069

0.252092

Thermal correction to Gibbs Free Energy=

Sum of electronic and zero-point Energies=

Sum of electronic and thermal Energies=

Sum of electronic and thermal Enthalpies=

0.197231

$-369.290353$

$-369.277224$

$-369.276200$

$-369.331062$
\end{abstract}

Cartesian Coordinates

$\begin{array}{lrrr}6 & -1.328299 & 2.422481 & -0.130850 \\ 6 & -0.194571 & 1.714754 & -0.260185 \\ 6 & -1.205419 & -0.478049 & -0.123927 \\ 6 & -1.525674 & -0.829499 & 1.322684 \\ 6 & -1.274008 & -1.690031 & -1.049738 \\ 1 & -2.004724 & 0.178310 & -0.483513 \\ 1 & -1.527425 & 0.067395 & 1.954465 \\ 1 & -2.517050 & -1.293912 & 1.391172 \\ 1 & -0.804849 & -1.541922 & 1.744487 \\ 1 & -0.895994 & -1.436874 & -2.046538 \\ 1 & -0.726291 & -2.563633 & -0.684700 \\ 1 & -2.322713 & -1.994745 & -1.156438 \\ 6 & 1.267785 & -0.123643 & 0.372707\end{array}$

Optimized Geometries from $(\mathrm{Me})_{2} \mathrm{NCH}_{2} \mathrm{CH}_{3}$ dehydrogenation

$\begin{array}{rrrr}6 & 2.455856 & 0.763549 & 0.014433 \\ 6 & 1.621287 & -1.554623 & -0.006393 \\ 1 & 1.142249 & -0.086118 & 1.472336 \\ 1 & 2.415002 & 1.760029 & 0.463690 \\ 1 & 3.375401 & 0.292636 & 0.378076 \\ 1 & 2.542131 & 0.872134 & -1.074907 \\ 1 & 0.913382 & -2.291547 & 0.380058 \\ 1 & 1.681350 & -1.665389 & -1.096782 \\ 1 & 2.601111 & -1.806937 & 0.414546 \\ 7 & 0.014478 & 0.338637 & -0.269604 \\ 1 & -1.278226 & 3.502587 & -0.233949 \\ 1 & -2.311705 & 2.000833 & 0.058078 \\ 1 & 0.715267 & 2.287058 & -0.425242\end{array}$

1

The smallest/imaginary frequencies is: $95.3650 \mathrm{~cm}(-1)$ 


$\begin{array}{lc}\text { Zero-point correction= } & 0.148017 \\ \text { Thermal correction to Energy= } & 0.155765 \\ \text { Thermal correction to Enthalpy= } & 0.156788 \\ \text { Thermal correction to Gibbs Free Energy= } & 0.115281 \\ \text { Sum of electronic and zero-point Energies }= & -213.477042 \\ \text { Sum of electronic and thermal Energies= } & -213.469294 \\ \text { Sum of electronic and thermal Enthalpies }= & -213.468270 \\ \text { Sum of electronic and thermal Free Energies }= & -213.509778\end{array}$

Cartesian Coordinates
$1 \quad 2.147523 \quad 1.220391-0.194613$

$6 \quad 1.049969-1.193573 \quad-0.205768$

$\begin{array}{lllll}1 & 0.732387 & -1.270761 & -1.265285\end{array}$

$\begin{array}{lllll}7 & 0.584183 & 0.000002 & 0.461744\end{array}$

$\begin{array}{llll}1 & 0.687326 & 2.085512 & 0.320690\end{array}$

$\begin{array}{lllll}1 & 0.732362 & 1.270772 & -1.265282\end{array}$

$\begin{array}{lllll}1 & 0.687329 & -2.085509 & 0.320683\end{array}$

$\begin{array}{llll}1 & 2.147535 & -1.220390 & -0.194600\end{array}$

4

The smallest/imaginary frequencies is: $24.8912 \mathrm{~cm}(-1)$

$\begin{array}{lc}\text { Zero-point correction }= & 0.765908 \\ \text { Thermal correction to Energy= } & 0.813918 \\ \text { Thermal correction to Enthalpy= } & 0.814941 \\ \text { Thermal correction to Gibbs Free Energy= } & 0.686959 \\ \text { Sum of electronic and zero-point Energies }= & -1939.704943 \\ \text { Sum of electronic and thermal Energies }= & -1939.656933 \\ \text { Sum of electronic and thermal Enthalpies }= & -1939.655909 \\ \text { Sum of electronic and thermal Free Energies }= & -1939.783892\end{array}$

Cartesian Coordinates

$\begin{array}{llll}1 & 2.581392 & -5.387825 & -1.280037 \\ 1 & 0.109484 & -5.144311 & -1.104101 \\ 1 & 3.429287 & -0.511960 & -1.898947 \\ 1 & 4.249073 & -0.994706 & -0.421119 \\ 1 & -1.815206 & -3.785952 & -0.326172 \\ 1 & -1.685219 & -2.873665 & -1.822072 \\ 6 & -3.640300 & -2.638559 & 1.838019 \\ 1 & -4.526938 & -2.046612 & 1.581804 \\ 1 & -3.614388 & -3.523632 & 1.189082 \\ 1 & -3.781131 & -2.997594 & 2.868324 \\ 6 & -4.295875 & -1.929529 & -1.368648 \\ 1 & -3.801510 & -2.753053 & -1.897179 \\ 1 & -4.803197 & -2.345063 & -0.492935 \\ 1 & -5.076314 & -1.541950 & -2.040197 \\ 6 & 4.386178 & 2.099998 & -1.498543\end{array}$




$\begin{array}{lrrrrrrr}1 & 5.061711 & 2.193680 & -0.642819 & 1 & -4.899536 & 0.692961 & -0.993467 \\ 1 & 4.669118 & 1.199993 & -2.056695 & 1 & -4.632503 & -0.045419 & 0.585562 \\ 1 & 4.577990 & 2.958886 & -2.158586 & 1 & -3.498975 & 1.177874 & -0.031906 \\ 6 & 4.594538 & 1.175459 & 1.731671 & 6 & 2.596149 & 3.417933 & -0.384458 \\ 1 & 5.246068 & 0.574335 & 1.084318 & 1 & 2.759756 & 4.253414 & -1.080803 \\ 1 & 4.705936 & 2.229769 & 1.451796 & 1 & 1.551905 & 3.473385 & -0.051325 \\ 1 & 4.977645 & 1.074308 & 2.757967 & 1 & 3.243423 & 3.591386 & 0.483641 \\ 6 & -1.208915 & -2.662484 & 2.340420 & 6 & 2.281563 & 1.623831 & 2.562513 \\ 1 & -1.091195 & -3.626011 & 1.829684 & 1 & 2.278476 & 2.663615 & 2.214696 \\ 1 & -0.251109 & -2.129775 & 2.281707 & 1 & 1.242272 & 1.268572 & 2.596537 \\ 1 & -1.420292 & -2.874738 & 3.398707 & 1 & 2.672132 & 1.623659 & 3.590915 \\ 6 & -2.763107 & -0.228751 & -2.322763 & 77 & 0.168988 & -0.104447 & -0.066113 \\ 1 & -2.210834 & -0.984261 & -2.897079 & 6 & -1.736277 & 3.267074 & -0.388760 \\ 1 & -3.585388 & 0.134581 & -2.957415 & 1 & -1.846697 & 2.540924 & -1.206870 \\ 1 & -2.068367 & 0.601131 & -2.139090 & 7 & -3.071768 & 3.709460 & -0.020362 \\ 6 & 2.057651 & 2.028435 & -2.365351 & 6 & -3.813947 & 4.109462 & -1.196532 \\ 1 & 2.210337 & 1.093559 & -2.920122 & 1 & -3.873810 & 3.273153 & -1.905339 \\ 1 & 0.985660 & 2.092775 & -2.136909 & 6 & -3.038421 & 4.784254 & 0.949325 \\ 1 & 2.323639 & 2.860487 & -3.034180 & 1 & -2.553696 & 4.459457 & 1.876645 \\ 6 & 3.067166 & -0.702645 & 2.281519 & 6 & -0.993739 & 2.603890 & 0.755076 \\ 1 & 3.719303 & -1.414568 & 1.760956 & 1 & -0.112825 & 2.055495 & 0.353391 \\ 1 & 3.390280 & -0.667890 & 3.332189 & 1 & -0.584839 & 3.316524 & 1.482398 \\ 1 & 2.043352 & -1.096495 & 2.249875 & 1 & -1.650837 & 1.911555 & 1.294922 \\ 6 & -2.489066 & -0.568755 & 2.628026 & 1 & -1.135096 & 4.110192 & -0.799167 \\ 1 & -2.743479 & -0.859640 & 3.658045 & 1 & -3.352545 & 4.970713 & -1.723427 \\ 1 & -1.542503 & -0.012089 & 2.660593 & 1 & -4.836039 & 4.393874 & -0.918926 \\ 1 & -3.275221 & 0.109611 & 2.275675 & 1 & -4.060707 & 5.090082 & 1.201075 \\ 6 & -4.119635 & 0.316404 & -0.314311 & 1 & -2.497857 & 5.678412 & 0.572945 \\ ------------------------------------------------------------------------- & \\ -\end{array}$

TS1

The smallest/imaginary frequencies is: $-599.0753 \mathrm{~cm}(-1)$

Zero-point correction=

Thermal correction to Energy=

Thermal correction to Enthalpy=

Thermal correction to Gibbs Free Energy=

Sum of electronic and zero-point Energies=

Sum of electronic and thermal Energies=

Sum of electronic and thermal Enthalpies=

Sum of electronic and thermal Free Energies=
0.760711

0.808571

0.809594

0.681943

$-1939.680634$

$-1939.632774$

$-1939.631750$

$-1939.759402$

Cartesian Coordinates

$\begin{array}{cccc}6 & 0.184955 & -1.933380 & -0.824097 \\ 15 & 2.368848 & -0.172382 & -0.000367 \\ 15 & -2.254933 & -0.564471 & 0.062581 \\ 6 & 2.695836 & -1.709275 & -0.987196 \\ 6 & 3.383921 & 1.124599 & -0.951022 \\ 6 & 3.150608 & -0.547933 & 1.689308 \\ 6 & -2.332732 & -2.182498 & -0.844908 \\ 6 & -2.920887 & -0.996391 & 1.789698\end{array}$

$\begin{array}{lrrr}6 & -3.505042 & 0.486290 & -0.918272 \\ 6 & 1.441722 & -2.532904 & -1.092219 \\ 6 & 1.551138 & -3.870754 & -1.475997 \\ 6 & 0.415428 & -4.657423 & -1.626642 \\ 6 & -0.952739 & -2.754971 & -1.021648 \\ 6 & -0.834446 & -4.092916 & -1.405355 \\ 1 & 2.537877 & -4.299091 & -1.657789 \\ 1 & 0.504031 & -5.702597 & -1.917387\end{array}$




$\begin{array}{lrrr}1 & -1.734161 & -4.696966 & -1.531612 \\ 1 & 3.002451 & -1.388291 & -1.992584 \\ 1 & 3.542782 & -2.284380 & -0.587018 \\ 1 & -3.015638 & -2.890013 & -0.353865 \\ 1 & -2.771489 & -1.987992 & -1.833211 \\ 6 & -4.391172 & -1.405361 & 1.823150 \\ 1 & -5.061829 & -0.551700 & 1.672625 \\ 1 & -4.639591 & -2.176090 & 1.082596 \\ 1 & -4.622837 & -1.822005 & 2.814323 \\ 6 & -4.854360 & -0.184702 & -1.184526 \\ 1 & -4.757898 & -1.187513 & -1.617871 \\ 1 & -5.476219 & -0.260221 & -0.288196 \\ 1 & -5.411575 & 0.425337 & -1.910848 \\ 6 & 4.799508 & 0.694172 & -1.339810 \\ 1 & 5.471722 & 0.607518 & -0.481527 \\ 1 & 4.822162 & -0.255996 & -1.886653 \\ 1 & 5.227213 & 1.456830 & -2.007383 \\ 6 & 4.672560 & -0.657451 & 1.671696 \\ 1 & 5.041638 & -1.349320 & 0.903858 \\ 1 & 5.156044 & 0.314986 & 1.522802 \\ 1 & 5.015502 & -1.040113 & 2.644159 \\ 6 & -2.072297 & -2.167151 & 2.294673 \\ 1 & -2.242532 & -3.090390 & 1.727472 \\ 1 & -1.000544 & -1.935937 & 2.249642 \\ 1 & -2.336265 & -2.372130 & 3.342287 \\ 6 & -2.841633 & 0.743548 & -2.277969 \\ 1 & -2.805770 & -0.161601 & -2.897655 \\ 1 & -3.426075 & 1.492717 & -2.831931 \\ 1 & -1.812130 & 1.108941 & -2.185770 \\ 6 & 2.597219 & 1.373960 & -2.243280 \\ 1 & 2.585081 & 0.492126 & -2.897004 \\ 1 & 1.553715 & 1.647169 & -2.046693 \\ 1 & 3.070304 & 2.191572 & -2.806397 \\ 6 & 2.566119 & -1.885605 & 2.151291 \\ 1 & 2.898172 & -2.728747 & 1.533291\end{array}$

$\begin{array}{crrr}1 & 2.898011 & -2.082437 & 3.180994 \\ 1 & 1.469141 & -1.872045 & 2.141930 \\ 6 & -2.711138 & 0.179467 & 2.743812 \\ 1 & -3.091575 & -0.093361 & 3.738981 \\ 1 & -1.647628 & 0.424626 & 2.850987 \\ 1 & -3.240920 & 1.086215 & 2.428862 \\ 6 & -3.756847 & 1.819761 & -0.214912 \\ 1 & -4.366416 & 2.462995 & -0.867014 \\ 1 & -4.321359 & 1.688432 & 0.716760 \\ 1 & -2.838281 & 2.371431 & 0.019832 \\ 6 & 3.467308 & 2.424779 & -0.152514 \\ 1 & 3.882038 & 3.219839 & -0.789549 \\ 1 & 2.486302 & 2.764633 & 0.201634 \\ 1 & 4.130820 & 2.330389 & 0.716417 \\ 6 & 2.738022 & 0.520352 & 2.702291 \\ 1 & 3.087125 & 1.523433 & 2.430031 \\ 1 & 1.648207 & 0.557208 & 2.822996 \\ 1 & 3.174808 & 0.276312 & 3.681645 \\ 77 & 0.034001 & -0.025306 & 0.036101 \\ 6 & -0.329155 & 2.989279 & -0.400234 \\ 1 & -0.990325 & 2.497381 & -1.122020 \\ 7 & -0.929030 & 4.296314 & -0.116763 \\ 6 & -1.310828 & 4.947586 & -1.351263 \\ 1 & -2.027160 & 4.322954 & -1.901700 \\ 6 & -0.033319 & 5.145287 & 0.639837 \\ 1 & 0.215634 & 4.688590 & 1.603875 \\ 6 & -0.160197 & 2.132887 & 0.850867 \\ 1 & -0.030262 & 0.736509 & 1.439959 \\ 1 & 0.705781 & 2.491196 & 1.425822 \\ 1 & -1.038504 & 2.322749 & 1.486127 \\ 1 & 0.645990 & 3.124891 & -0.915038 \\ 1 & -0.512468 & 6.110668 & 0.843623 \\ 1 & 0.917003 & 5.345262 & 0.098186 \\ 1 & -0.445772 & 5.143870 & -2.020105\end{array}$

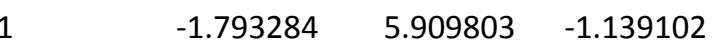

5

The smallest/imaginary frequencies is: $26.6277 \mathrm{~cm}(-1)$

$\begin{array}{lc}\text { Zero-point correction= } & 0.761795 \\ \text { Thermal correction to Energy= } & 0.809698 \\ \text { Thermal correction to Enthalpy= } & 0.810722 \\ \text { Thermal correction to Gibbs Free Energy= } & 0.684043 \\ \text { Sum of electronic and zero-point Energies }= & -1939.691630 \\ \text { Sum of electronic and thermal Energies }= & -1939.643726 \\ \text { Sum of electronic and thermal Enthalpies }= & -1939.642703 \\ \text { Sum of electronic and thermal Free Energies }= & -1939.769381\end{array}$

Cartesian Coordinates 


$\begin{array}{llll}6 & 0.191317 & -2.044370 & -0.676149 \\ 15 & 2.362744 & -0.187746 & -0.024428 \\ 15 & -2.253997 & -0.584945 & 0.028194 \\ 6 & 2.690415 & -1.805175 & -0.879822 \\ 6 & 3.317440 & 1.054939 & -1.090016 \\ 6 & 3.161175 & -0.408341 & 1.680477 \\ 6 & -2.318360 & -2.264404 & -0.767925 \\ 6 & -2.972739 & -0.885132 & 1.757623 \\ 6 & -3.428640 & 0.435486 & -1.057365 \\ 6 & 1.450719 & -2.661273 & -0.875392 \\ 6 & 1.567868 & -4.031541 & -1.116307 \\ 6 & 0.433638 & -4.832572 & -1.196076 \\ 6 & -0.943570 & -2.878942 & -0.820606 \\ 6 & -0.821442 & -4.249205 & -1.061790 \\ 1 & 2.556380 & -4.475045 & -1.246521 \\ 1 & 0.526977 & -5.902438 & -1.373248 \\ 1 & -1.718515 & -4.864475 & -1.148884 \\ 1 & 2.952567 & -1.562751 & -1.919990 \\ 1 & 3.569099 & -2.320231 & -0.465949 \\ 1 & -3.058743 & -2.917224 & -0.284193 \\ 1 & -2.682871 & -2.117291 & -1.794648 \\ 1 & -2.556135 & -2.212637 & 3.399158 \\ 1 & -2.647890 & 0.666489 & -2.357552 \\ 6 & -4.467273 & -1.197567 & 1.749297 \\ 1 & -5.071547 & -0.320093 & 1.491470 \\ 1 & -4.728444 & -2.013926 & 1.063806 \\ 1 & -4.771663 & -1.511409 & 2.758324 \\ 6 & -4.750664 & -0.243613 & -1.417593 \\ 1 & -4.614978 & -1.231100 & -1.874644 \\ 1 & -5.418080 & -0.356119 & -0.558542 \\ 1 & -5.277143 & 0.380685 & -2.154533 \\ 6 & 4.699807 & 0.593269 & -1.551563 \\ 1 & 5.406774 & 0.475108 & -0.724997 \\ 1 & 4.669263 & -0.349667 & -2.110012 \\ 1 & 5.117773 & 1.352824 & -2.228517 \\ 6 & 4.686459 & -0.462026 & 1.633690 \\ 1 & 5.062526 & -1.217745 & 0.932150 \\ 1 & 5.129268 & 0.506044 & 1.371587 \\ 1 & 5.065259 & -0.729224 & 2.630870 \\ 1 & -2.229594 & -2.082825 & 2.357527 \\ 1 & & & -2.910269\end{array}$

\begin{tabular}{|c|c|c|c|}
\hline 1 & -3.221975 & 1.333922 & -3.016374 \\
\hline 1 & -1.671849 & 1.137257 & -2.180179 \\
\hline J & 2.435237 & 1.251512 & -2.328690 \\
\hline & 2.295778 & 0.321178 & -2.895726 \\
\hline & 1.442224 & 1.640099 & -2.063644 \\
\hline & 2.905845 & 1.979731 & -3.004871 \\
\hline & 2.646848 & -1.731494 & 2.255647 \\
\hline$\perp$ & 2.990478 & -2.604031 & 1.687341 \\
\hline 1 & 3.025013 & -1.839177 & 3.282216 \\
\hline & 1.551594 & -1.768858 & 2.297296 \\
\hline כ & -2.718500 & 0.328750 & 2.650715 \\
\hline 1 & -3.131692 & 0.130398 & 3.650396 \\
\hline 1 & -1.646696 & 0.531024 & 2.762713 \\
\hline 1 & -3.194429 & 1.241369 & 2.273414 \\
\hline b & -3.726344 & 1.782251 & -0.398210 \\
\hline 1 & -4.242356 & 2.433065 & -1.119812 \\
\hline 1 & -4.393208 & 1.671998 & 0.466303 \\
\hline 1 & -2.824604 & 2.310150 & -0.065418 \\
\hline 6 & 3.454714 & 2.389114 & -0.357789 \\
\hline$\perp$ & 3.815486 & 3.154440 & -1.060534 \\
\hline 1 & 2.500852 & 2.740735 & 0.054167 \\
\hline 1 & 4.183461 & 2.333724 & 0.460564 \\
\hline 6 & 2.722898 & 0.719699 & 2.614410 \\
\hline 1 & 3.056073 & 1.706408 & 2.272611 \\
\hline 1 & 1.632148 & 0.752241 & 2.725384 \\
\hline 1 & 3.160070 & 0.551766 & 3.609388 \\
\hline 77 & 0.024701 & -0.026662 & -0.031135 \\
\hline 6 & -0.337484 & 3.108600 & -0.404428 \\
\hline 1 & -1.030621 & 2.750938 & -1.182764 \\
\hline 7 & -0.899135 & 4.385644 & 0.055809 \\
\hline 6 & -1.236141 & 5.225777 & -1.070539 \\
\hline 1 & -1.965918 & 4.717499 & -1.715358 \\
\hline 6 & 0.010663 & 5.079268 & 0.940281 \\
\hline 1 & 0.231460 & 4.468339 & 1.821727 \\
\hline 6 & -0.154475 & 2.057730 & 0.681068 \\
\hline 1 & 0.075526 & -0.410284 & 1.458394 \\
\hline 1 & 0.715570 & 2.333249 & 1.298234 \\
\hline 1 & -1.021113 & 2.149042 & 1.355278 \\
\hline 1 & 0.626018 & 3.304585 & -0.932858 \\
\hline 1 & -1.686687 & 6.165347 & -0.725955 \\
\hline 1 & -0.353528 & 5.485306 & -1.695241 \\
\hline 1 & 0.975378 & 5.327749 & 0.443867 \\
\hline
\end{tabular}

The smallest/imaginary frequencies is: $-740.3383 \mathrm{~cm}(-1)$

Zero-point correction=

Thermal correction to Energy=

Thermal correction to Enthalpy=
0.758995

0.805627

0.806651 
Thermal correction to Gibbs Free Energy= Sum of electronic and zero-point Energies= Sum of electronic and thermal Energies= Sum of electronic and thermal Enthalpies= Sum of electronic and thermal Free Energies=

\author{
0.683725 \\ $-1939.666025$ \\ $-1939.619392$ \\ $-1939.618369$ \\ $-1939.741295$
}

Cartesian Coordinates

\begin{tabular}{|c|c|c|c|}
\hline 6 & 0.050407 & -1.781359 & -0.602042 \\
\hline 15 & 2.333880 & -0.233089 & 0.255396 \\
\hline 15 & -2.337640 & -0.277294 & 0.040960 \\
\hline 6 & 2.321657 & -2.075273 & 0.387256 \\
\hline 6 & 3.320440 & 0.056901 & -1.372894 \\
\hline 6 & 3.381917 & 0.315851 & 1.750113 \\
\hline 6 & -2.361230 & -1.617004 & -1.243612 \\
\hline 6 & -3.063285 & -1.088721 & 1.610980 \\
\hline 6 & -3.615580 & 0.935419 & -0.700002 \\
\hline 6 & 1.193493 & -2.598595 & -0.454563 \\
\hline 6 & 1.254017 & -3.885265 & -0.990539 \\
\hline 6 & 0.152255 & -4.426043 & -1.646252 \\
\hline 6 & -1.071110 & -2.381886 & -1.205661 \\
\hline 6 & -1.016656 & -3.678463 & -1.725045 \\
\hline 1 & 2.164558 & -4.474929 & -0.871430 \\
\hline 1 & 0.196233 & -5.429160 & -2.065965 \\
\hline 1 & -1.905291 & -4.105288 & -2.192972 \\
\hline 1 & 3.287022 & -2.540164 & 0.157808 \\
\hline 1 & 2.102486 & -2.293677 & 1.442729 \\
\hline 1 & -3.236239 & -2.273838 & -1.132749 \\
\hline 1 & -2.476244 & -1.104688 & -2.210202 \\
\hline 6 & -4.559970 & -1.387169 & 1.544597 \\
\hline 1 & -5.173698 & -0.479823 & 1.542274 \\
\hline 1 & -4.831777 & -2.000316 & 0.676156 \\
\hline 1 & -4.841300 & -1.958517 & 2.441055 \\
\hline 6 & -4.890916 & 0.261292 & -1.216380 \\
\hline 1 & -4.683759 & -0.505395 & -1.972099 \\
\hline 1 & -5.505950 & -0.187501 & -0.434267 \\
\hline 1 & -5.506755 & 1.029775 & -1.705888 \\
\hline 6 & 4.390158 & -1.016288 & -1.597633 \\
\hline 1 & 5.088008 & -1.125903 & -0.761014 \\
\hline 1 & 3.946045 & -1.996354 & -1.809073 \\
\hline 1 & 4.982730 & -0.737237 & -2.480799 \\
\hline 6 & 4.778816 & -0.302744 & 1.763755 \\
\hline 1 & 4.753607 & -1.398714 & 1.716190 \\
\hline 1 & 5.408629 & 0.065530 & 0.945488 \\
\hline 1 & 5.281971 & -0.031072 & 2.703360 \\
\hline 6 & -2.347151 & -2.423152 & 1.840987 \\
\hline 1 & -2.570186 & -3.157355 & 1.056996 \\
\hline 1 & -1.259891 & -2.309203 & 1.903135 \\
\hline 1 & -2.697333 & -2.843935 & 2.794650 \\
\hline 6 & -2.950081 & 1.618822 & -1.896021 \\
\hline 1 & -2.584669 & 0.908439 & -2.648609 \\
\hline
\end{tabular}

$\begin{array}{lccc}1 & -3.694503 & 2.260231 & -2.390546 \\ 1 & -2.120439 & 2.253774 & -1.573427 \\ 6 & 2.353068 & -0.019239 & -2.557045 \\ 1 & 1.785668 & -0.956343 & -2.575680 \\ 1 & 1.631326 & 0.804000 & -2.553209 \\ 1 & 2.938510 & 0.042654 & -3.486282 \\ 6 & 2.637491 & -0.133652 & 3.011496 \\ 1 & 2.589022 & -1.224169 & 3.111074 \\ 1 & 3.176501 & 0.246254 & 3.890946 \\ 1 & 1.611828 & 0.252068 & 3.043306 \\ 6 & -2.794656 & -0.178025 & 2.810273 \\ 1 & -3.168997 & -0.664778 & 3.722701 \\ 1 & -1.722875 & 0.011584 & 2.938758 \\ 1 & -3.303303 & 0.790041 & 2.724238 \\ 6 & -3.986008 & 2.016467 & 0.313515 \\ 1 & -4.623158 & 2.767742 & -0.175612 \\ 1 & -4.549300 & 1.622210 & 1.167870 \\ 1 & -3.099124 & 2.540539 & 0.692827 \\ 6 & 3.992421 & 1.429380 & -1.407950 \\ 1 & 4.377602 & 1.606014 & -2.422659 \\ 1 & 3.297098 & 2.248116 & -1.180953 \\ 1 & 4.847409 & 1.498683 & -0.725786 \\ 6 & 3.512184 & 1.839856 & 1.818780 \\ 1 & 4.133564 & 2.255546 & 1.021494 \\ 1 & 2.540008 & 2.343854 & 1.789811 \\ 1 & 3.989294 & 2.109664 & 2.771925 \\ 77 & -0.006181 & 0.168694 & 0.240894 \\ 6 & 0.305587 & 2.441795 & -0.122280 \\ 1 & 0.114107 & 1.268258 & -1.128765 \\ 1 & -0.441037 & 3.394105 & -0.899366 \\ 6 & 0.037806 & 3.493102 & -2.262786 \\ 1 & 1.102771 & 3.799131 & -2.316225 \\ 6 & -0.427050 & 4.700619 & -0.260437 \\ 1 & -0.849133 & 4.641301 & 0.746822 \\ 6 & -0.115012 & 2.134266 & 1.217373 \\ 1 & -0.037977 & -0.704964 & 1.612934 \\ 1 & 0.605127 & 2.248151 & 2.026022 \\ 1 & -1.122949 & 2.447850 & 1.494226 \\ 1 & 1.395417 & 2.567061 & -0.252544 \\ 1 & 0.596502 & 5.120131 & -0.181512 \\ 1 & -0.066726 & 2.526126 & -2.771360\end{array}$


TS1'

The smallest/imaginary frequencies is: $-530.9415 \mathrm{~cm}(-1)$

$\begin{array}{lc}\text { Zero-point correction }= & 0.761433 \\ \text { Thermal correction to Energy= } & 0.808811 \\ \text { Thermal correction to Enthalpy= } & 0.809835 \\ \text { Thermal correction to Gibbs Free Energy= } & 0.684384 \\ \text { Sum of electronic and zero-point Energies }= & -1939.673466 \\ \text { Sum of electronic and thermal Energies= } & -1939.626088 \\ \text { Sum of electronic and thermal Enthalpies }= & -1939.625065 \\ \text { Sum of electronic and thermal Free Energies }= & -1939.750515\end{array}$

Cartesian Coordinates

\begin{tabular}{|c|c|c|c|c|c|c|c|}
\hline 6 & 0.123157 & 2.013990 & -0.149569 & 6 & -2.995285 & -0.267241 & -2.583499 \\
\hline 15 & 2.356011 & 0.131790 & 0.108227 & 1 & -3.146111 & 0.729310 & -3.016240 \\
\hline 15 & -2.283726 & 0.406315 & -0.011686 & 1 & -1.922506 & -0.478728 & -2.619116 \\
\hline 6 & 2.547011 & 1.929883 & 0.518924 & 1 & -3.507658 & -0.981593 & -3.243929 \\
\hline 6 & 3.226977 & -0.693489 & 1.591627 & 6 & -2.104236 & 1.507420 & 2.528952 \\
\hline 6 & 3.417886 & -0.049693 & -1.463556 & 1 & -2.306961 & 2.525770 & 2.174642 \\
\hline 6 & -2.359911 & 2.180242 & -0.550889 & 1 & -2.372481 & 1.470121 & 3.595023 \\
\hline 6 & -3.588126 & -0.363349 & -1.168748 & 1 & -1.025888 & 1.325193 & 2.439695 \\
\hline 6 & -2.919190 & 0.447805 & 1.782092 & 6 & 2.231118 & -0.648897 & 2.754424 \\
\hline 6 & 1.338943 & 2.703428 & 0.078559 & 1 & 1.899129 & 0.371638 & 2.987068 \\
\hline 6 & 1.426556 & 4.094058 & -0.004841 & 1 & 1.334886 & -1.243891 & 2.545270 \\
\hline 6 & 0.296585 & 4.855936 & -0.278491 & 1 & 2.712528 & -1.054855 & 3.656250 \\
\hline 6 & -1.013376 & 2.824137 & -0.383785 & 6 & 2.835263 & 0.945597 & -2.471713 \\
\hline 6 & -0.925006 & 4.217088 & -0.447385 & 1 & 2.968277 & 1.988975 & -2.160317 \\
\hline 1 & 2.385805 & 4.584057 & 0.168190 & 1 & 3.349292 & 0.820690 & -3.435782 \\
\hline 1 & 0.365039 & 5.940500 & -0.340614 & 1 & 1.762141 & 0.782139 & -2.635301 \\
\hline 1 & -1.825626 & 4.803858 & -0.634110 & 6 & -3.817378 & -1.824259 & -0.783735 \\
\hline 1 & 2.613410 & 1.989362 & 1.614593 & 1 & -4.402336 & -2.325688 & -1.569146 \\
\hline 1 & 3.487072 & 2.351665 & 0.136093 & 1 & -2.882585 & -2.380998 & -0.633597 \\
\hline 1 & -2.649066 & 2.179990 & -1.610801 & 1 & -4.397803 & -1.903131 & 0.145209 \\
\hline 1 & -3.147441 & 2.737661 & -0.022350 & 6 & -2.645274 & -0.902195 & 2.443642 \\
\hline 6 & -4.940320 & 0.353247 & -1.217552 & 1 & -3.049734 & -0.893806 & 3.466617 \\
\hline 1 & -5.544015 & 0.200589 & -0.319739 & 1 & -3.101060 & -1.745095 & 1.909778 \\
\hline 1 & -4.844729 & 1.432691 & -1.388435 & 1 & -1.566733 & -1.085876 & 2.508216 \\
\hline 1 & -5.515214 & -0.053974 & -2.062865 & 6 & 3.561139 & -2.154180 & 1.296125 \\
\hline 6 & -4.400201 & 0.784706 & 1.931514 & 1 & 3.880336 & -2.647379 & 2.225947 \\
\hline 1 & -4.692138 & 1.691282 & 1.386667 & 1 & 2.698358 & -2.711804 & 0.910164 \\
\hline 1 & -5.047005 & -0.039263 & 1.609679 & 1 & 4.385960 & -2.254266 & 0.580104 \\
\hline 1 & -4.616897 & 0.962751 & 2.995022 & 6 & 3.283758 & -1.452905 & -2.053430 \\
\hline 6 & 4.507146 & 0.015295 & 2.041886 & 1 & 3.688241 & -2.231730 & -1.397792 \\
\hline 1 & 5.289731 & 0.018038 & 1.278173 & 1 & 2.242724 & -1.704013 & -2.278897 \\
\hline 1 & 4.333368 & 1.051323 & 2.353583 & 1 & 3.845670 & -1.498257 & -2.997914 \\
\hline 1 & 4.911176 & -0.516159 & 2.915927 & 77 & 0.025505 & -0.088581 & -0.1164 \\
\hline 6 & 4.901641 & 0.255950 & -1.273857 & 6 & 0.057798 & -2.384328 & -0.545304 \\
\hline 1 & 5.084883 & 1.221993 & -0.787316 & 1 & 1.111403 & -2.703581 & -0.404032 \\
\hline 1 & 5.415116 & -0.524840 & -0.700463 & 7 & -0.808212 & -3.368929 & 0.130203 \\
\hline 1 & 5.385040 & 0.296167 & -2.261138 & 6 & -0.368456 & -3.632595 & 1.4840 \\
\hline
\end{tabular}




$\begin{array}{lrrr}1 & -0.351178 & -2.712438 & 2.079343 \\ 6 & -0.878853 & -4.641855 & -0.570581 \\ 1 & -1.357462 & -4.540885 & -1.548891 \\ 6 & -0.225894 & -2.368061 & -2.042365 \\ 1 & 0.219491 & -3.240276 & -2.547971 \\ 1 & -1.302162 & -2.385390 & -2.243187\end{array}$

$\begin{array}{rrrr}1 & -0.038874 & -1.434861 & 0.743006 \\ 1 & 0.181686 & -1.472517 & -2.525824 \\ 1 & 0.120824 & -5.103064 & -0.718789 \\ 1 & -1.483592 & -5.342693 & 0.016922 \\ 1 & 0.650210 & -4.075884 & 1.519890 \\ 1 & -1.056005 & -4.331567 & 1.975697\end{array}$

$5^{\prime}$

The smallest/imaginary frequencies is: $25.3616 \mathrm{~cm}(-1)$

Zero-point correction=

Thermal correction to Energy=

Thermal correction to Enthalpy=

Thermal correction to Gibbs Free Energy=

Sum of electronic and zero-point Energies=

Sum of electronic and thermal Energies=

Sum of electronic and thermal Enthalpies=

Sum of electronic and thermal Free Energies=
0.763024

0.810555

0.811578

0.687105

$-1939.676358$

$-1939.628828$

$-1939.627804$

$-1939.752277$

Cartesian Coordinates

$\begin{array}{cccc}6 & 0.006328 & 1.959757 & -0.220677 \\ 15 & -2.346034 & 0.267296 & -0.042659 \\ 15 & 2.328146 & 0.245338 & 0.108348 \\ 6 & -2.394671 & 1.902411 & -0.916294 \\ 6 & -3.420243 & -0.796974 & -1.203122 \\ 6 & -3.234724 & 0.579873 & 1.600548 \\ 6 & 2.383843 & 2.049193 & 0.546806 \\ 6 & 3.556219 & -0.436899 & 1.407710 \\ 6 & 3.105960 & 0.224883 & -1.642470 \\ 6 & -1.148319 & 2.676860 & -0.605723 \\ 6 & -1.159635 & 4.069123 & -0.713659 \\ 6 & -0.010924 & 4.804634 & -0.444464 \\ 6 & 1.144673 & 2.742372 & 0.067592 \\ 6 & 1.141699 & 4.133985 & -0.051778 \\ 1 & -2.076088 & 4.579467 & -1.014409 \\ 1 & -0.016133 & 5.889424 & -0.532963 \\ 1 & 2.048427 & 4.696616 & 0.176896 \\ 1 & -2.425505 & 1.668833 & -1.990411 \\ 1 & -3.314300 & 2.464093 & -0.698265 \\ 1 & 2.426128 & 2.090865 & 1.645323 \\ 1 & 3.303105 & 2.532119 & 0.185219 \\ 6 & 4.785665 & 0.448552 & 1.634953 \\ 1 & 5.445389 & 0.505012 & 0.765021 \\ 1 & 4.527690 & 1.469491 & 1.935925 \\ 1 & 5.373917 & 0.014761 & 2.456705 \\ 6 & 4.586605 & 0.595280 & -1.668594 \\ 1 & 4.792693 & 1.564167 & -1.197450 \\ 1 & 5.216501 & -0.165421 & -1.193403 \\ 1 & 4.912901 & 0.671409 & -2.716174 \\ 6 & -4.585554 & -0.032210 & -1.838412\end{array}$

$\begin{array}{lrrr}1 & -5.289671 & 0.367346 & -1.101180 \\ 1 & -4.257119 & 0.793149 & -2.478812 \\ 1 & -5.147045 & -0.727570 & -2.479410 \\ 6 & -4.716071 & 0.916211 & 1.440282 \\ 1 & -4.882207 & 1.781641 & 0.786372 \\ 1 & -5.303868 & 0.074330 & 1.057831 \\ 1 & -5.129213 & 1.174806 & 2.425970 \\ 6 & 2.779120 & -0.494172 & 2.726222 \\ 1 & 2.453286 & 0.498860 & 3.061812 \\ 1 & 1.892229 & -1.130751 & 2.655974 \\ 1 & 3.432134 & -0.903625 & 3.510469 \\ 6 & 2.345903 & 1.233990 & -2.509313 \\ 1 & 2.444279 & 2.266558 & -2.156223 \\ 1 & 2.755206 & 1.190344 & -3.529050 \\ 1 & 1.274167 & 1.005618 & -2.569178 \\ 6 & -2.467231 & -1.239239 & -2.320179 \\ 1 & -2.032572 & -0.379486 & -2.851626 \\ 1 & -1.637222 & -1.855939 & -1.949206 \\ 1 & -3.024696 & -1.826755 & -3.064874 \\ 6 & -2.546394 & 1.766778 & 2.279668 \\ 1 & -2.652760 & 2.698328 & 1.710751 \\ 1 & -3.010442 & 1.924267 & 3.263921 \\ 1 & -1.475971 & 1.590708 & 2.436216 \\ 6 & 4.034991 & -1.843289 & 1.059086 \\ 1 & 4.685708 & -2.211579 & 1.865372 \\ 1 & 3.207721 & -2.554070 & 0.962662 \\ 1 & 4.623540 & -1.869317 & 0.133354 \\ 6 & 2.930669 & -1.145850 & -2.290667 \\ 1 & 3.490461 & -1.934227 & -1.774818 \\ 1 & 1.874641 & -1.444446 & -2.334301 \\ & & & \end{array}$




$\begin{array}{cccc}1 & 3.306130 & -1.102913 & -3.323540 \\ 6 & -4.001736 & -2.027556 & -0.506385 \\ 1 & -4.423693 & -2.701775 & -1.266565 \\ 1 & -3.258683 & -2.597276 & 0.059699 \\ 1 & -4.818891 & -1.765098 & 0.176138 \\ 6 & -3.080716 & -0.644611 & 2.502461 \\ 1 & -3.585778 & -1.531727 & 2.103886 \\ 1 & -2.024513 & -0.895145 & 2.660014 \\ 1 & -3.525417 & -0.427293 & 3.484303 \\ 77 & -0.025242 & -0.171972 & 0.092311 \\ 6 & -0.338411 & -2.340106 & 0.508678 \\ 1 & -1.427537 & -2.421436 & 0.624023 \\ 7 & -0.059237 & -3.173454 & -0.698294\end{array}$

$\begin{array}{lrrr}6 & -0.965042 & -4.295495 & -0.860479 \\ 1 & -2.000427 & -3.951763 & -0.971326 \\ 6 & 1.299334 & -3.656149 & -0.773951 \\ 1 & 1.988773 & -2.836237 & -0.566946 \\ 6 & 0.216097 & -2.959649 & 1.788833 \\ 1 & -0.150302 & -2.401641 & 2.662063 \\ 1 & -0.092480 & -4.010793 & 1.931347 \\ 1 & 1.312771 & -2.945663 & 1.844067 \\ 1 & -0.059780 & 0.075465 & 1.617619 \\ 1 & 1.519431 & -4.473881 & -0.057009 \\ 1 & 1.517767 & -4.038331 & -1.781768 \\ 1 & -0.707468 & -4.855024 & -1.770756 \\ 1 & -0.933179 & -5.012186 & -0.014555\end{array}$

TS2'

The smallest/imaginary frequencies is: $-651.5016 \mathrm{~cm}(-1)$

$\begin{array}{lc}\text { Zero-point correction= } & 0.759717 \\ \text { Thermal correction to Energy= } & 0.806026 \\ \text { Thermal correction to Enthalpy= } & 0.807049 \\ \text { Thermal correction to Gibbs Free Energy= } & 0.685127 \\ \text { Sum of electronic and zero-point Energies }= & -1939.664878 \\ \text { Sum of electronic and thermal Energies= } & -1939.618569 \\ \text { Sum of electronic and thermal Enthalpies }= & -1939.617545 \\ \text { Sum of electronic and thermal Free Energies }= & -1939.739467\end{array}$

Cartesian Coordinates

$\begin{array}{lrrr}1 & 4.348279 & 2.381591 & 1.084517 \\ 1 & 4.431585 & 1.871350 & 2.770478 \\ 6 & 4.809142 & 0.595976 & -1.363803 \\ 1 & 4.445096 & 1.312810 & -2.108848 \\ 1 & 5.245651 & 1.158856 & -0.535615 \\ 1 & 5.625838 & 0.031971 & -1.837580 \\ 6 & -4.722359 & 1.029835 & -1.163402 \\ 1 & -5.354416 & 0.767485 & -0.310852 \\ 1 & -4.413066 & 2.076747 & -1.055333 \\ 1 & -5.352492 & 0.977014 & -2.063005 \\ 6 & -4.742892 & -0.713034 & 1.692263 \\ 1 & -4.898651 & 0.357962 & 1.872872 \\ 1 & -5.352021 & -1.014996 & 0.832926 \\ 1 & -5.138919 & -1.247433 & 2.567931 \\ 6 & 1.878418 & 2.008015 & 2.274301 \\ 1 & 1.933033 & 2.953098 & 1.721453 \\ 1 & 0.833710 & 1.679498 & 2.286474 \\ 1 & 2.182062 & 2.212234 & 3.311448 \\ 6 & 3.186857 & -1.051777 & -2.216850 \\ 1 & 2.571568 & -0.374076 & -2.823439 \\ 1 & 4.036414 & -1.368302 & -2.839168 \\ 1 & 2.604019 & -1.942845 & -1.968129 \\ 6 & -2.695052 & 0.704432 & -2.505087\end{array}$




$\begin{array}{lrrrcrll}1 & -2.249160 & 1.669348 & -2.236067 & 1 & -3.456559 & -2.853287 & 0.228125 \\ 1 & -1.887196 & 0.044044 & -2.833117 & 1 & -2.105104 & -2.905757 & 1.379185 \\ 1 & -3.363302 & 0.870068 & -3.362799 & 1 & -3.764113 & -3.123801 & 1.947512 \\ 6 & -2.577291 & -0.830104 & 2.876267 & 77 & -0.055462 & -0.240200 & -0.152993 \\ 1 & -2.786475 & 0.163018 & 3.290288 & 6 & -0.087655 & -1.827975 & -1.751720 \\ 1 & -2.965230 & -1.564060 & 3.596906 & 1 & -0.010582 & -0.231667 & -1.861393 \\ 1 & -1.490075 & -0.961031 & 2.813698 & 6 & 0.046527 & -2.438913 & -0.457004 \\ 6 & 2.743899 & -0.272099 & 2.645021 & 1 & -0.037660 & -0.174960 & 1.487751 \\ 1 & 3.089010 & 0.030683 & 3.644305 & 1 & -0.854233 & -2.960948 & -0.100713 \\ 1 & 1.712240 & -0.629704 & 2.741700 & 1 & -1.052575 & -1.918201 & -2.252322 \\ 1 & 3.369465 & -1.111008 & 2.321344 & 1 & 0.738016 & -1.915028 & -2.455956 \\ 6 & 4.344383 & -1.513640 & -0.098634 & 7 & 1.260271 & -3.153824 & -0.139262 \\ 1 & 5.070995 & -2.059745 & -0.718463 & 6 & 1.309272 & -3.451332 & 1.276661 \\ 1 & 4.888105 & -1.140870 & 0.776274 & 1 & 1.208680 & -2.524229 & 1.851171 \\ 1 & 3.582116 & -2.233222 & 0.226237 & 6 & 1.371711 & -4.389852 & -0.895331 \\ 6 & -4.006835 & -1.277280 & -1.798174 & 1 & 2.323642 & -4.887401 & -0.663533 \\ 1 & -4.488871 & -1.191532 & -2.782858 & 1 & 0.490736 & -4.135488 & 1.588475 \\ 1 & -3.185875 & -1.999306 & -1.901028 & 1 & 2.260724 & -3.935550 & 1.537738 \\ 1 & -4.749196 & -1.701841 & -1.112256 & 1 & 0.550006 & -5.101209 & -0.667355 \\ 6 & -3.136998 & -2.567844 & 1.235633 & 1 & 1.351931 & -4.187308 & -1.972462 \\ --------------------------------------------------------------------------------------------------------- & \end{array}$

6

The smallest/imaginary frequencies is: $37.5991 \mathrm{~cm}(-1)$

$\begin{array}{lc}\text { Zero-point correction }= & 0.763518 \\ \text { Thermal correction to Energy= } & 0.809905 \\ \text { Thermal correction to Enthalpy= } & 0.810929 \\ \text { Thermal correction to Gibbs Free Energy= } & 0.689319 \\ \text { Sum of electronic and zero-point Energies }= & -1939.682922 \\ \text { Sum of electronic and thermal Energies= } & -1939.636534 \\ \text { Sum of electronic and thermal Enthalpies }= & -1939.635511 \\ \text { Sum of electronic and thermal Free Energies }= & -1939.757120\end{array}$

Cartesian Coordinates

$\begin{array}{cccccccc}6 & -0.087414 & 1.854867 & -0.375220 & 1 & 1.787110 & 4.353135 & -1.776601 \\ 15 & -2.359024 & 0.148141 & 0.205239 & 1 & -3.358264 & 2.430783 & 0.393583 \\ 15 & 2.326260 & 0.307147 & 0.000093 & 1 & -2.189877 & 2.036344 & 1.659477 \\ 6 & -2.387208 & 1.957651 & 0.581183 & 1 & 3.175472 & 2.429830 & -0.968439 \\ 6 & -3.329119 & 0.034150 & -1.447698 & 1 & 2.373518 & 1.383957 & -2.141949 \\ 6 & -3.414365 & -0.596569 & 1.618996 & 6 & 4.566623 & 1.271927 & 1.571036 \\ 6 & 2.299901 & 1.782359 & -1.119140 & 1 & 5.183317 & 0.371783 & 1.471523 \\ 6 & 3.072614 & 0.960719 & 1.635158 & 1 & 4.821543 & 1.966252 & 0.760668 \\ 6 & 3.592797 & -0.810569 & -0.887972 & 1 & 4.861214 & 1.756721 & 2.513031 \\ 6 & -1.257514 & 2.610223 & -0.158631 & 6 & 4.854476 & -0.076113 & -1.351678 \\ 6 & -1.351760 & 3.941708 & -0.565641 & 1 & 4.627853 & 0.778086 & -2.000614 \\ 6 & -0.265934 & 4.571907 & -1.164659 & 1 & 5.490936 & 0.272674 & -0.536250 \\ 6 & 1.008224 & 2.525273 & -0.945898 & 1 & 5.455250 & -0.777054 & -1.949138 \\ 6 & 0.917722 & 3.864414 & -1.334517 & 6 & -4.497143 & 1.022250 & -1.524303 \\ 1 & -2.279381 & 4.490433 & -0.396957 & 1 & -5.210151 & 0.940992 & -0.699623 \\ 1 & -0.337438 & 5.610597 & -1.481273 & 1 & -4.144719 & 2.059847 & -1.575853\end{array}$




$\begin{array}{lrrrrrrr}1 & -5.052858 & 0.830053 & -2.453332 & 1 & 4.540807 & -1.675039 & 0.896891 \\ 6 & -4.866935 & -0.120044 & 1.603856 & 1 & 3.101034 & -2.559558 & 0.321614 \\ 1 & -4.952283 & 0.973496 & 1.580388 & 6 & -3.844052 & -1.386674 & -1.673892 \\ 1 & -5.439380 & -0.534651 & 0.766428 & 1 & -4.223069 & -1.469628 & -2.702657 \\ 1 & -5.357759 & -0.461693 & 2.526944 & 1 & -3.048807 & -2.136344 & -1.560590 \\ 6 & 2.350142 & 2.258591 & 2.009009 & 1 & -4.671859 & -1.650126 & -1.005224 \\ 1 & 2.559615 & 3.073618 & 1.305691 & 6 & -3.402376 & -2.127322 & 1.589527 \\ 1 & 1.264734 & 2.125825 & 2.070647 & 1 & -3.784645 & -2.549296 & 0.655431 \\ 1 & 2.707803 & 2.579405 & 2.998080 & 1 & -2.402374 & -2.536883 & 1.764502 \\ 6 & 2.909513 & -1.370248 & -2.136742 & 1 & -4.048131 & -2.496895 & 2.399155 \\ 1 & 2.582370 & -0.587268 & -2.831686 & 77 & 0.002826 & -0.189460 & 0.154706 \\ 1 & 3.632364 & -2.001950 & -2.673923 & 6 & -0.220475 & -2.440103 & 0.078850 \\ 1 & 2.051483 & -1.985805 & -1.861008 & 1 & 0.068119 & -0.422707 & -1.497452 \\ 6 & -2.390450 & 0.379459 & -2.606825 & 7 & 0.595343 & -3.240453 & -0.780458 \\ 1 & -1.924012 & 1.365019 & -2.490734 & 6 & 0.013533 & -3.368448 & -2.100685 \\ 1 & -1.587817 & -0.354752 & -2.719915 & 1 & -0.114382 & -2.375227 & -2.547517 \\ 1 & -2.982049 & 0.393646 & -3.533879 & 6 & 0.832451 & -4.560037 & -0.213974 \\ 6 & -2.795517 & -0.160483 & 2.951971 & 1 & 1.298808 & -4.479168 & 0.772918 \\ 1 & -2.979909 & 0.897209 & 3.172762 & 6 & 0.228462 & -2.041552 & 1.349080 \\ 1 & -3.262256 & -0.741297 & 3.760045 & 1 & -0.028377 & 0.419026 & 1.717580 \\ 1 & -1.713001 & -0.328853 & 2.988856 & 1 & -0.450065 & -2.026041 & 2.199260 \\ 6 & 2.825410 & -0.055882 & 2.750342 & 1 & 1.267360 & -2.236327 & 1.615291 \\ 1 & 3.225105 & 0.342425 & 3.694044 & 1 & -1.295240 & -2.636090 & -0.050337 \\ 1 & 1.751738 & -0.236498 & 2.882247 & 1 & -0.102601 & -5.146542 & -0.103990 \\ 1 & 3.321302 & -1.017029 & 2.565686 & 1 & 1.510649 & -5.124220 & -0.866624 \\ 6 & 3.981982 & -1.986402 & 0.006016 & 1 & 0.672588 & -3.961331 & -2.747188 \\ 1 & 4.629964 & -2.671459 & -0.559966 & 1 & -0.976377 & -3.869427 & -2.074422 \\ ------------------------------------------------------------------------------------------------- & \\ & & & & & & \end{array}$

enamine

The smallest/imaginary frequencies is: $140.8505 \mathrm{~cm}(-1)$

Zero-point correction=

Thermal correction to Energy=

Thermal correction to Enthalpy=

Thermal correction to Gibbs Free Energy=

Sum of electronic and zero-point Energies=

Sum of electronic and thermal Energies=

Sum of electronic and thermal Enthalpies=

Sum of electronic and thermal Free Energies=
0.124583

0.131919

0.132942

0.092396

$-212.275585$

$-212.268249$

$-212.267226$

$-212.307772$

Cartesian Coordinates

$\begin{array}{llrl}6 & 1.993433 & -0.114877 & 0.114043 \\ 6 & 0.793809 & -0.669846 & -0.113097 \\ 6 & -0.448485 & 1.395645 & -0.003008 \\ 1 & -0.231724 & 1.645043 & 1.051378 \\ 6 & -1.609475 & -0.744969 & 0.132290 \\ 1 & -1.795983 & -0.714484 & 1.219954 \\ 7 & -0.417853 & -0.023223 & -0.254567\end{array}$

$\begin{array}{lrrr}1 & 2.879179 & -0.742029 & 0.126972 \\ 1 & 2.137628 & 0.951069 & 0.268731 \\ 1 & 0.715376 & -1.751535 & -0.239920 \\ 1 & -1.438650 & 1.789836 & -0.254883 \\ 1 & 0.290968 & 1.906234 & -0.631272 \\ 1 & -1.521952 & -1.795332 & -0.166448 \\ 1 & -2.485558 & -0.321957 & -0.373911\end{array}$


$\mathrm{N}$-methylpiperidine (Optimized with keyword int=superfine)

1

The smallest/imaginary frequencies is: $166.1682 \mathrm{~cm}(-1)$

Zero-point correction=

Thermal correction to Energy=

Thermal correction to Enthalpy=

Thermal correction to Gibbs Free Energy=

Sum of electronic and zero-point Energies=

Sum of electronic and thermal Energies=

Sum of electronic and thermal Enthalpies=

Sum of electronic and thermal Free Energies=
0.185800 (Hartree/Particle)

0.193799

0.194822

0.152319

$-290.814965$

$-290.806966$

$-290.805943$

$-290.848446$

\section{Cartesian Coordinates}

\begin{tabular}{|c|c|c|c|c|c|c|c|}
\hline 6 & 0.314680 & 1.199515 & 0.176037 & 1 & -2.921796 & 0.000000 & -0.075063 \\
\hline 6 & 0.314680 & -1.199514 & 0.176037 & 1 & -1.895528 & 0.000000 & 1.361004 \\
\hline 6 & -1.149141 & -1.246098 & -0.227900 & 1 & -1.209590 & 1.302599 & -1.324816 \\
\hline 6 & -1.876529 & 0.000000 & 0.258957 & 1 & -1.613896 & 2.156538 & 0.171655 \\
\hline 6 & -1.149141 & 1.246098 & -0.227901 & 1 & 0.392501 & -1.244844 & 1.287579 \\
\hline 1 & 0.852203 & -2.074904 & -0.214295 & 6 & 2.370455 & -0.000000 & 0.000849 \\
\hline 1 & 0.392501 & 1.244844 & 1.287579 & 1 & 2.859367 & -0.887388 & -0.419858 \\
\hline 1 & 0.852203 & 2.074905 & -0.214295 & 1 & 2.859367 & 0.887388 & -0.419858 \\
\hline 1 & -1.209591 & -1.302599 & -1.324816 & 1 & 2.552797 & 0.000000 & 1.096417 \\
\hline 1 & -1.613896 & -2.156538 & 0.171655 & 7 & 0.964763 & 0.000000 & -0.331337 \\
\hline
\end{tabular}

TS1

The smallest/imaginary frequencies is: $-562.8567 \mathrm{~cm}(-1)$

Zero-point correction=

Thermal correction to Energy=

Thermal correction to Enthalpy=

0.798454 (Hartree/Particle)

0.846832

0.847856

Thermal correction to Gibbs Free Energy=

Sum of electronic and zero-point Energies=

Sum of electronic and thermal Energies=

Sum of electronic and thermal Enthalpies=

0.719944

$-2017.012682$

$-2016.964304$

$-2016.963280$

Sum of electronic and thermal Free Energies=

Cartesian Coordinates

$\begin{array}{cccc}6 & 0.048413 & -2.038124 & -0.892928 \\ 15 & 2.334966 & -0.438131 & -0.003255 \\ 15 & -2.312464 & -0.563116 & 0.028165 \\ 6 & 2.549042 & -1.879896 & -1.147071 \\ 6 & 3.634745 & 0.791119 & -0.686024 \\ 6 & 2.919677 & -1.094129 & 1.684945 \\ 6 & -2.466907 & -2.202254 & -0.832928\end{array}$

$\begin{array}{llll}6 & -2.920122 & -0.947263 & 1.792530 \\ 6 & -3.625135 & 0.457699 & -0.910105 \\ 6 & 1.274912 & -2.673379 & -1.208260 \\ 6 & 1.333606 & -4.015945 & -1.585120 \\ 6 & 0.170980 & -4.772477 & -1.678351 \\ 6 & -1.116670 & -2.826494 & -1.038957 \\ 6 & -1.052677 & -4.171956 & -1.411284\end{array}$




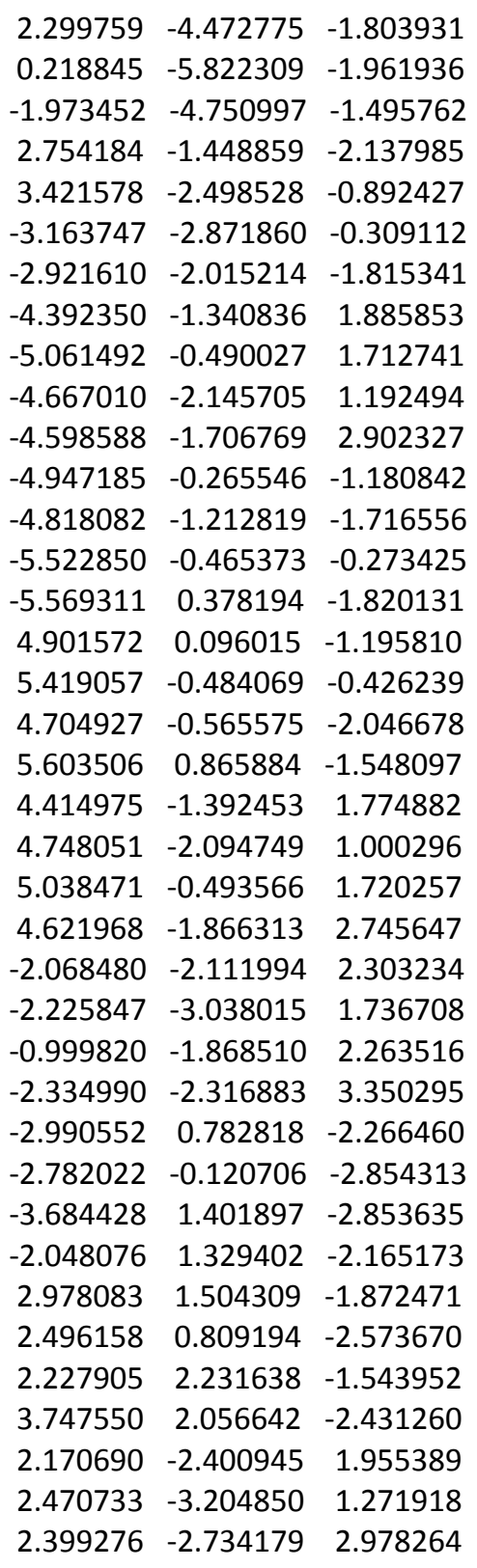

$\begin{array}{cccc}1 & 1.085570 & -2.276461 & 1.868345 \\ 6 & -2.671006 & 0.247793 & 2.712033 \\ 1 & -3.001841 & -0.006414 & 3.729758 \\ 1 & -1.604533 & 0.499217 & 2.761057 \\ 1 & -3.220617 & 1.145125 & 2.404494 \\ 6 & -3.934784 & 1.755460 & -0.166923 \\ 1 & -4.524124 & 2.418582 & -0.817074 \\ 1 & -4.531618 & 1.576404 & 0.736369 \\ 1 & -3.030188 & 2.302113 & 0.125560 \\ 6 & 4.048229 & 1.851020 & 0.336526 \\ 1 & 4.676607 & 2.599528 & -0.167983 \\ 1 & 3.194332 & 2.390362 & 0.763698 \\ 1 & 4.640959 & 1.438154 & 1.160636 \\ 6 & 2.530567 & -0.087553 & 2.769724 \\ 1 & 2.994632 & 0.895950 & 2.627259 \\ 1 & 1.441973 & 0.051773 & 2.805550 \\ 1 & 2.855176 & -0.465607 & 3.750204 \\ 77 & -0.001815 & -0.097662 & -0.062581 \\ 6 & -0.233636 & 2.808840 & -0.853460 \\ 1 & -1.340955 & 2.801890 & -0.937295 \\ 7 & 0.249542 & 4.192109 & -0.908728 \\ 6 & 0.196287 & 2.127295 & 0.437996 \\ 1 & -0.076061 & 0.787911 & 1.263976 \\ 1 & 1.283290 & 2.243245 & 0.512525 \\ 1 & 0.153877 & 2.278295 & -1.734659 \\ 6 & -0.387438 & 2.915419 & 1.615289 \\ 1 & -0.052936 & 2.496027 & 2.576944 \\ 1 & -1.488161 & 2.837420 & 1.612382 \\ 6 & 0.018752 & 4.383473 & 1.524225 \\ 1 & -0.458909 & 4.972130 & 2.319182 \\ 1 & 1.106970 & 4.467595 & 1.667881 \\ 6 & -0.338399 & 4.970334 & 0.170382 \\ 1 & 0.017356 & 6.007446 & 0.090742 \\ 1 & -1.448682 & 5.003580 & 0.062905 \\ 6 & -0.051247 & 4.781943 & -2.193246 \\ 1 & 0.330102 & 5.809860 & -2.238091 \\ 1 & 0.430229 & 4.206441 & -2.994102 \\ 1 & -1.141319 & 4.815356 & -2.405513 \\ & & & \end{array}$

The smallest/imaginary frequencies is: $22.0201 \mathrm{~cm}(-1)$
Zero-point correction=
0.799501 (Hartree/Particle)
Thermal correction to Energy=
0.848000
Thermal correction to Enthalpy=
0.849023
Thermal correction to Gibbs Free Energy= $\quad 0.721152$
Sum of electronic and zero-point Energies $=\quad-2017.021541$
Sum of electronic and thermal Energies= $\quad-2016.973042$
Sum of electronic and thermal Enthalpies $=\quad-2016.972019$
Sum of electronic and thermal Free Energies $=\quad-2017.099890$ 
Cartesian Coordinates

\begin{tabular}{|c|c|c|c|c|c|c|c|}
\hline 6 & 0.654817 & -2.110460 & -0.102421 & 6 & 2.192105 & 1.612738 & -2.409655 \\
\hline 15 & 2.441672 & 0.169428 & -0.090869 & 1 & 1.982460 & 0.650140 & -2.898282 \\
\hline 15 & -2.025860 & -1.069194 & 0.224028 & 1 & 1.241192 & 2.044628 & -2.077373 \\
\hline 6 & 2.914553 & -1.416323 & -0.937484 & 1 & 2.611812 & 2.279798 & -3.176207 \\
\hline 6 & 3.191900 & 1.474600 & -1.255830 & 6 & 3.066645 & -1.209415 & 2.241891 \\
\hline 6 & 3.385306 & 0.121289 & 1.554821 & 1 & 3.445708 & -2.074198 & 1.684011 \\
\hline 6 & -1.568501 & -2.778970 & 0.797253 & 1 & 3.549346 & -1.215545 & 3.229834 \\
\hline 6 & -3.440312 & -0.648809 & 1.430586 & 1 & 1.990423 & -1.352222 & 2.390466 \\
\hline 6 & -2.682972 & -1.347060 & -1.554048 & 6 & -4.027291 & 0.742832 & 1.198778 \\
\hline 6 & 1.923454 & -2.493185 & -0.588553 & 1 & -4.817054 & 0.916895 & 1.943870 \\
\hline 6 & 2.265104 & -3.835954 & -0.770921 & 1 & -3.273030 & 1.524320 & 1.332929 \\
\hline 6 & 1.346953 & -4.840407 & -0.484752 & 1 & -4.479090 & 0.872481 & 0.211192 \\
\hline 6 & -0.242570 & -3.156077 & 0.205552 & 6 & -3.755231 & -0.331035 & -1.945093 \\
\hline 6 & 0.089744 & -4.496681 & 0.004144 & 1 & -3.992381 & -0.465485 & -3.010405 \\
\hline 1 & 3.255433 & -4.095853 & -1.148479 & 1 & -4.689689 & -0.471659 & -1.389761 \\
\hline 1 & 1.610516 & -5.885447 & -0.636783 & 1 & -3.422874 & 0.706651 & -1.814422 \\
\hline 1 & -0.634015 & -5.277315 & 0.244196 & 6 & 3.347496 & 2.832837 & -0.570628 \\
\hline 1 & 2.870539 & -1.205549 & -2.016845 & 1 & 3.591360 & 3.589306 & -1.330534 \\
\hline 1 & 3.953392 & -1.709828 & -0.726858 & 1 & 2.432675 & 3.164142 & -0.065029 \\
\hline 1 & -1.464311 & -2.703599 & 1.888768 & 1 & 4.165058 & 2.836762 & 0.160010 \\
\hline 1 & -2.351112 & -3.523264 & 0.604635 & 6 & 2.895775 & 1.251457 & 2.459911 \\
\hline 6 & -4.565650 & -1.681374 & 1.375344 & 1 & 3.060015 & 2.246921 & 2.031898 \\
\hline 1 & -5.099930 & -1.663752 & 0.417463 & 1 & 1.826245 & 1.149858 & 2.678524 \\
\hline 1 & -4.213573 & -2.704326 & 1.554400 & 1 & 3.438886 & 1.208779 & 3.415102 \\
\hline 1 & -5.302224 & -1.451687 & 2.159209 & 77 & 0.101150 & -0.052373 & 0.078306 \\
\hline 6 & -3.236149 & -2.752959 & -1.778612 & 6 & -1.428961 & 2.524703 & -0.812916 \\
\hline 1 & -2.475000 & -3.527282 & -1.626127 & 1 & -2.391944 & 2.053517 & -0.517821 \\
\hline 1 & -4.094313 & -2.977552 & -1.133537 & 7 & -1.625575 & 3.977288 & -0.912803 \\
\hline 1 & -3.580133 & -2.838460 & -2.819830 & 6 & -0.332660 & 2.109932 & 0.155481 \\
\hline 6 & 4.545034 & 1.070785 & -1.847764 & 1 & 0.239458 & -0.007703 & 1.613962 \\
\hline 1 & 5.320361 & 0.923594 & -1.089962 & 1 & 0.568938 & 2.626654 & -0.213871 \\
\hline 1 & 4.486381 & 0.161391 & -2.456350 & 1 & -1.208846 & 2.162612 & -1.833025 \\
\hline 1 & 4.889962 & 1.877217 & -2.511223 & 6 & -0.613578 & 2.738701 & 1.521368 \\
\hline 6 & 4.901295 & 0.218254 & 1.393154 & 1 & 0.221577 & 2.550132 & 2.212663 \\
\hline 1 & 5.303652 & -0.551362 & 0.722295 & 1 & -1.498994 & 2.272063 & 1.988183 \\
\hline 1 & 5.227925 & 1.200075 & 1.032795 & 6 & -0.854684 & 4.242540 & 1.406344 \\
\hline 1 & 5.370923 & 0.065048 & 2.375481 & 1 & -1.131492 & 4.682170 & 2.375731 \\
\hline 6 & -2.829792 & -0.642052 & 2.837155 & 1 & 0.073899 & 4.737138 & 1.079760 \\
\hline 1 & -2.558031 & -1.642025 & 3.192860 & 6 & -1.944685 & 4.537347 & 0.391015 \\
\hline 1 & -1.939014 & -0.001921 & 2.892331 & 1 & -2.093040 & 5.621614 & 0.278256 \\
\hline 1 & -3.570099 & -0.242160 & 3.544141 & 1 & -2.913175 & 4.124784 & 0.763749 \\
\hline 6 & -1.480400 & -1.144005 & -2.486185 & 6 & -2.675252 & 4.274765 & -1.857565 \\
\hline 1 & -0.715821 & -1.917157 & -2.354926 & 1 & -2.811902 & 5.359687 & -1.954567 \\
\hline 1 & -1.820101 & -1.178306 & -3.532482 & 1 & -2.420448 & 3.875345 & -2.847770 \\
\hline 1 & -1.007478 & -0.157127 & -2.336586 & 1 & -3.654701 & 3.837115 & -1.559199 \\
\hline
\end{tabular}

TS2

The smallest/imaginary frequencies is: $-767.3092 \mathrm{~cm}(-1)$ 


$\begin{array}{lc}\text { Zero-point correction= } & 0.795820 \text { (Hartree/Particle) } \\ \text { Thermal correction to Energy= } & 0.843923 \\ \text { Thermal correction to Enthalpy= } & 0.844946 \\ \text { Thermal correction to Gibbs Free Energy= } & 0.719845 \\ \text { Sum of electronic and zero-point Energies }= & -2016.994696 \\ \text { Sum of electronic and thermal Energies }= & -2016.946593 \\ \text { Sum of electronic and thermal Enthalpies }= & -2016.945570 \\ \text { Sum of electronic and thermal Free Energies }= & -2017.070671\end{array}$

Cartesian Coordinates

$\begin{array}{ccc}-2.756142 & 0.423053 & -2.633567 \\ -4.036277 & 1.597278 & -2.282423 \\ -2.438374 & 1.753173 & -1.511467 \\ 2.977736 & 1.484323 & -2.215673 \\ 3.211109 & 0.660039 & -2.900787 \\ 1.888776 & 1.591955 & -2.218725 \\ 3.421473 & 2.393172 & -2.646628 \\ 2.679715 & -1.845187 & 2.167648 \\ 3.135902 & -2.626327 & 1.547700 \\ 2.976421 & -2.039672 & 3.208163 \\ 1.591865 & -1.951679 & 2.104169 \\ -2.490370 & -0.586242 & 2.814097 \\ -2.709753 & -1.093297 & 3.765135 \\ -1.464008 & -0.201423 & 2.862809 \\ -3.174753 & 0.267746 & 2.734070 \\ -4.344322 & 1.139514 & 0.358886 \\ -5.124739 & 1.736550 & -0.136366 \\ -4.827119 & 0.603817 & 1.184026 \\ -3.625480 & 1.844504 & 0.787605 \\ 3.611367 & 2.551968 & -0.029003 \\ 4.198878 & 3.298965 & -0.582588 \\ 2.617639 & 2.978733 & 0.144563 \\ 4.094234 & 2.423726 & 0.947856 \\ 2.588652 & 0.553759 & 2.801425 \\ 2.904500 & 1.585245 & 2.599473 \\ 1.492441 & 0.527733 & 2.825896 \\ 2.960186 & 0.290092 & 3.802344 \\ 0.102089 & 0.074508 & -0.021978 \\ 0.010028 & 2.508795 & -0.541360 \\ -0.101452 & 1.240534 & -1.397090 \\ -1.006639 & 3.316480 & -1.028127 \\ 0.033136 & 2.114954 & 0.857570 \\ 0.210073 & -0.710218 & 1.379883 \\ 1.004884 & 2.315622 & 1.316845 \\ 0.979111 & 2.738577 & -0.992889 \\ -1.096003 & 2.640286 & 1.720491 \\ -0.789050 & 2.623711 & 2.775407 \\ -1.981124 & 1.995582 & 1.661228 \\ -1.471976 & 4.052705 & 1.285636 \\ -2.277787 & 4.472220 & 1.902516 \\ -0.597686 & 4.711688 & 1.399007 \\ -1.919027 & 4.052392 & -0.165642\end{array}$




$\begin{array}{llllllll}6 & -0.990340 & 3.651154 & -2.426926 & 1 & -0.693777 & 2.770998 & -3.016818 \\ 1 & -1.995212 & 3.945147 & -2.757571 & 1 & -1.978153 & 5.086644 & -0.538635 \\ 1 & -0.296203 & 4.475237 & -2.664722 & 1 & -2.941454 & 3.635864 & -0.247585\end{array}$

TS1'

The smallest/imaginary frequencies is: $-582.5545 \mathrm{~cm}(-1)$

Zero-point correction=

Thermal correction to Energy=

Thermal correction to Enthalpy=

Thermal correction to Gibbs Free Energy=

Sum of electronic and zero-point Energies=

Sum of electronic and thermal Energies=

Sum of electronic and thermal Enthalpies=

Sum of electronic and thermal Free Energies=
0.799428 (Hartree/Particle)

0.847331

0.848354

0.722515

$-2017.006219$

$-2016.958316$

$-2016.957292$

$-2017.083131$

Cartesian Coordinates

$\begin{array}{cccc}6 & 0.421043 & -1.978693 & -0.822216 \\ 15 & 2.406047 & -0.016485 & -0.005732 \\ 15 & -2.146532 & -0.846511 & 0.059653 \\ 6 & 2.880601 & -1.476449 & -1.042620 \\ 6 & 3.572481 & 1.336893 & -0.681772 \\ 6 & 2.989833 & -0.505623 & 1.746112 \\ 6 & -2.045053 & -2.510157 & -0.754749 \\ 6 & -2.525171 & -1.275781 & 1.883984 \\ 6 & -3.735968 & -0.150232 & -0.744385 \\ 6 & 1.732882 & -2.445691 & -1.085801 \\ 6 & 1.992041 & -3.785670 & -1.379119 \\ 6 & 0.953886 & -4.708044 & -1.433211 \\ 6 & -0.614980 & -2.937422 & -0.923805 \\ 6 & -0.348423 & -4.278748 & -1.209710 \\ 1 & 3.018102 & -4.108945 & -1.560117 \\ 1 & 1.158580 & -5.754851 & -1.650410 \\ 1 & -1.172716 & -4.991468 & -1.261601 \\ 1 & 3.072257 & -1.084600 & -2.052649 \\ 1 & 3.817213 & -1.947025 & -0.710059 \\ 1 & -2.649996 & -3.264730 & -0.231344 \\ 1 & -2.501898 & -2.397587 & -1.748629 \\ 6 & -3.889795 & -1.918513 & 2.122330 \\ 1 & -4.718006 & -1.216281 & 1.975934 \\ 1 & -4.062156 & -2.800301 & 1.492635 \\ 1 & -3.940294 & -2.256657 & 3.167691 \\ 6 & -4.871052 & -1.165002 & -0.905096 \\ 1 & -4.586771 & -2.019933 & -1.529746 \\ 1 & -5.259187 & -1.549755 & 0.040338 \\ 1 & -5.707481 & -0.665483 & -1.415823 \\ 6 & 4.982780 & 0.839465 & -1.009814 \\ 1 & 5.528805 & 0.462143 & -0.142115 \\ 1 & 4.979308 & 0.057342 & -1.777995 \\ 1 & 5.563354 & 1.681128 & -1.415398\end{array}$

$\begin{array}{lrrr}6 & 4.503084 & -0.506593 & 1.954524 \\ 1 & 5.023424 & -1.155232 & 1.238835 \\ 1 & 4.947898 & 0.493128 & 1.908908 \\ 1 & 4.715538 & -0.904490 & 2.957761 \\ 6 & -1.451574 & -2.260483 & 2.347186 \\ 1 & -1.474822 & -3.209937 & 1.797987 \\ 1 & -0.451517 & -1.828991 & 2.237130 \\ 1 & -1.611514 & -2.488941 & 3.411010 \\ 6 & -3.333559 & 0.323944 & -2.141589 \\ 1 & -2.787100 & -0.436292 & -2.716844 \\ 1 & -4.238725 & 0.580424 & -2.711191 \\ 1 & -2.712289 & 1.217827 & -2.068860 \\ 6 & 2.947243 & 1.831909 & -1.987091 \\ 1 & 2.703698 & 1.016927 & -2.682018 \\ 1 & 2.029479 & 2.396083 & -1.802919 \\ 1 & 3.654640 & 2.501455 & -2.497580 \\ 6 & 2.501089 & -1.928834 & 2.023901 \\ 1 & 3.042807 & -2.676657 & 1.431823 \\ 1 & 2.674628 & -2.161339 & 3.084643 \\ 1 & 1.434468 & -2.052135 & 1.815492 \\ 6 & -2.414128 & -0.024950 & 2.755784 \\ 1 & -2.598998 & -0.299563 & 3.804841 \\ 1 & -1.411120 & 0.414171 & 2.696824 \\ 1 & -3.143210 & 0.748023 & 2.487144 \\ 6 & -4.243099 & 1.064458 & 0.029681 \\ 1 & -5.039763 & 1.554314 & -0.549811 \\ 1 & -4.671403 & 0.797116 & 1.003394 \\ 1 & -3.444322 & 1.804318 & 0.176640 \\ 6 & 3.668252 & 2.521712 & 0.280580 \\ 1 & 4.186530 & 3.352205 & -0.220996 \\ 1 & 2.681929 & 2.894144 & 0.588717 \\ 1 & 4.239663 & 2.286961 & 1.185939 \\ 6 & 2.342130 & 0.438408 & 2.759638\end{array}$




$\begin{array}{cccccccc}1 & 2.643434 & 1.483448 & 2.614901 & 1 & 0.183301 & 4.948932 & 1.024182 \\ 1 & 1.247589 & 0.387553 & 2.700177 & 6 & -1.801943 & 4.813451 & 0.204992 \\ 1 & 2.644124 & 0.147538 & 3.776523 & 1 & -1.925748 & 5.895273 & 0.066502 \\ 77 & 0.057470 & -0.001354 & -0.194230 & 1 & -2.785819 & 4.407723 & 0.485692 \\ 6 & -0.236813 & 2.318837 & 0.045307 & 6 & -1.340873 & 4.195006 & -1.096106 \\ 1 & -0.247045 & 1.050043 & 0.967357 & 1 & -0.394905 & 4.698828 & -1.403221 \\ 1 & 0.798883 & 2.640022 & -0.176867 & 1 & -2.068584 & 4.386471 & -1.898200 \\ 6 & -0.641038 & 2.984031 & 1.371744 & 7 & -1.130209 & 2.744504 & -1.034684 \\ 1 & 0.101729 & 2.710134 & 2.136747 & 6 & -0.647694 & 2.344470 & -2.346952 \\ 1 & -1.603056 & 2.562455 & 1.707501 & 1 & -0.402499 & 1.274879 & -2.352224 \\ 6 & -0.786800 & 4.493733 & 1.284976 & 1 & -1.414498 & 2.540239 & -3.108943 \\ 1 & -1.084044 & 4.907996 & 2.257285 & 1 & 0.255316 & 2.913016 & -2.645503\end{array}$

$5^{\prime}$

The smallest/imaginary frequencies is: $20.3605 \mathrm{~cm}(-1)$

$\begin{array}{lc}\text { Zero-point correction= } & 0.801124 \text { (Hartree/Particle) } \\ \text { Thermal correction to Energy= } & 0.849108 \\ \text { Thermal correction to Enthalpy= } & 0.850132 \\ \text { Thermal correction to Gibbs Free Energy= } & 0.724879 \\ \text { Sum of electronic and zero-point Energies }= & -2017.016601 \\ \text { Sum of electronic and thermal Energies= } & -2016.968616 \\ \text { Sum of electronic and thermal Enthalpies }= & -2016.967593 \\ \text { Sum of electronic and thermal Free Energies }= & -2017.092845\end{array}$

Cartesian Coordinates

$\begin{array}{llll}1 & -4.021640 & -2.318799 & 3.043364 \\ 6 & -4.875016 & -1.032357 & -0.992930 \\ 1 & -4.580840 & -1.833136 & -1.681071 \\ 1 & -5.273781 & -1.493019 & -0.086428 \\ 1 & -5.706179 & -0.494574 & -1.472181 \\ 6 & 5.010093 & 0.744850 & -1.018233 \\ 1 & 5.559306 & 0.360379 & -0.155759 \\ 1 & 4.982703 & -0.039214 & -1.783944 \\ 1 & 5.601515 & 1.574092 & -1.433196 \\ 6 & 4.503225 & -0.602228 & 1.934309 \\ 1 & 4.982088 & -1.260758 & 1.199474 \\ 1 & 4.985248 & 0.380149 & 1.892839 \\ 1 & 4.719708 & -1.022200 & 2.927245 \\ 6 & -1.517219 & -2.316255 & 2.282624 \\ 1 & -1.515594 & -3.221414 & 1.663378 \\ 1 & -0.509478 & -1.891292 & 2.262471 \\ 1 & -1.731593 & -2.622425 & 3.316662 \\ 6 & -3.311519 & 0.538825 & -2.091795 \\ 1 & -2.759293 & -0.186247 & -2.707014 \\ 1 & -4.203624 & 0.836613 & -2.661760 \\ 1 & -2.689047 & 1.423639 & -1.936595 \\ 6 & 2.984761 & 1.786220 & -1.971629 \\ 1 & 2.713631 & 0.978712 & -2.666252 \\ 1 & 2.086867 & 2.373431 & -1.764917\end{array}$




$\begin{array}{lrrrrrrr}1 & 3.700071 & 2.439239 & -2.491875 & 77 & 0.059945 & 0.056172 & -0.193941 \\ 6 & 2.444831 & -1.937907 & 2.059070 & 6 & -0.189828 & 2.223514 & 0.036786 \\ 1 & 2.956302 & -2.712316 & 1.474694 & 1 & 0.056337 & 0.105244 & 1.343603 \\ 1 & 2.614076 & -2.162889 & 3.121984 & 1 & 0.820917 & 2.684074 & -0.082617 \\ 1 & 1.374316 & -2.027072 & 1.855912 & 6 & -0.720962 & 2.747537 & 1.366492 \\ 6 & -2.472570 & -0.095779 & 2.793078 & 1 & -0.058447 & 2.398160 & 2.173757 \\ 1 & -2.673422 & -0.437120 & 3.819153 & 1 & -1.712549 & 2.310492 & 1.566935 \\ 1 & -1.474355 & 0.356709 & 2.782960 & 6 & -0.842662 & 4.267454 & 1.414030 \\ 1 & -3.201509 & 0.688701 & 2.561329 & 1 & -1.259742 & 4.602639 & 2.374287 \\ 6 & -4.276137 & 1.131304 & 0.099759 & 1 & 0.161685 & 4.716448 & 1.330039 \\ 1 & -5.057984 & 1.656923 & -0.468051 & 6 & -1.698661 & 4.743172 & 0.252023 \\ 1 & -4.729878 & 0.801640 & 1.042143 & 1 & -1.751713 & 5.839552 & 0.212231 \\ 1 & -3.486577 & 1.861405 & 0.319480 & 1 & -2.729203 & 4.375420 & 0.375007 \\ 6 & 3.743046 & 2.456828 & 0.285976 & 6 & -1.134229 & 4.215501 & -1.052695 \\ 1 & 4.273520 & 3.275806 & -0.221501 & 1 & -0.131111 & 4.676764 & -1.220974 \\ 1 & 2.765233 & 2.846894 & 0.598437 & 1 & -1.766012 & 4.524143 & -1.900474 \\ 1 & 4.318629 & 2.210852 & 1.185611 & 7 & -1.023824 & 2.762236 & -1.066814 \\ 6 & 2.415995 & 0.438380 & 2.793787 & 6 & -0.530565 & 2.365674 & -2.367001 \\ 1 & 2.692553 & 1.480665 & 2.595462 & 1 & -0.318655 & 1.284246 & -2.394212 \\ 1 & 1.321652 & 0.385388 & 2.844126 & 1 & -1.274572 & 2.573148 & -3.150563 \\ 1 & 2.805092 & 0.177018 & 3.788484 & 1 & 0.398149 & 2.898294 & -2.655122 \\ ------------------------------------------------------------------------------------------------ & \end{array}$

TS2'

The smallest/imaginary frequencies is: $-749.1981 \mathrm{~cm}(-1)$

$\begin{array}{lc}\text { Zero-point correction= } & 0.796013 \text { (Hartree/Particle) } \\ \text { Thermal correction to Energy= } & 0.843677 \\ \text { Thermal correction to Enthalpy= } & 0.844700 \\ \text { Thermal correction to Gibbs Free Energy= } & 0.721003 \\ \text { Sum of electronic and zero-point Energies }= & -2016.987973 \\ \text { Sum of electronic and thermal Energies }= & -2016.940309 \\ \text { Sum of electronic and thermal Enthalpies }= & -2016.939286 \\ \text { Sum of electronic and thermal Free Energies }= & -2017.062983\end{array}$

Cartesian Coordinates

$\begin{array}{crrrrrrr}6 & -0.365302 & 1.953298 & -0.749646 & 1 & -0.848811 & 5.835913 & -1.283228 \\ 15 & -2.462154 & 0.042077 & 0.017808 & 1 & 1.429386 & 4.826993 & -1.323734 \\ 15 & 2.180246 & 0.692836 & -0.043612 & 1 & -3.260767 & 1.522690 & -1.695914 \\ 6 & -2.869686 & 1.699978 & -0.684110 & 1 & -3.684361 & 2.183363 & -0.126573 \\ 6 & -3.664665 & -1.066149 & -0.984170 & 1 & 2.873339 & 2.972896 & -0.721521 \\ 6 & -3.060273 & 0.156639 & 1.831415 & 1 & 2.342044 & 1.963358 & -2.066086 \\ 6 & 2.103998 & 2.251568 & -1.031180 & 6 & 4.112990 & 1.824684 & 1.791242 \\ 6 & 2.674804 & 1.316020 & 1.695703 & 1 & 4.855079 & 1.023850 & 1.705677 \\ 6 & 3.688471 & -0.156830 & -0.850086 & 1 & 4.341081 & 2.598076 & 1.047576 \\ 6 & -1.642524 & 2.556051 & -0.783011 & 1 & 4.252411 & 2.282491 & 2.781251 \\ 6 & -1.811578 & 3.931299 & -0.965367 & 6 & 4.867797 & 0.767570 & -1.169762 \\ 6 & -0.716374 & 4.763919 & -1.150774 & 1 & 4.579553 & 1.626788 & -1.786346 \\ 6 & 0.721806 & 2.824592 & -0.980033 & 1 & 5.387746 & 1.139400 & -0.284593 \\ 6 & 0.551755 & 4.197272 & -1.168728 & 1 & 5.601302 & 0.190549 & -1.752379 \\ 1 & -2.820261 & 4.347800 & -0.966206 & 6 & -5.106466 & -0.547232 & -1.063745\end{array}$




$\begin{array}{lrrr}1 & -5.666790 & -0.674117 & -0.135762 \\ 1 & -5.167904 & 0.505968 & -1.361388 \\ 1 & -5.633863 & -1.125750 & -1.836266 \\ 6 & -4.578912 & 0.175303 & 1.997200 \\ 1 & -5.066353 & 0.948462 & 1.390586 \\ 1 & -5.039489 & -0.792519 & 1.771082 \\ 1 & -4.810732 & 0.398027 & 3.048629 \\ 6 & 1.762072 & 2.485884 & 2.074630 \\ 1 & 1.948515 & 3.377372 & 1.464149 \\ 1 & 0.699994 & 2.234112 & 1.990706 \\ 1 & 1.963039 & 2.756612 & 3.121337 \\ 6 & 3.189804 & -0.698172 & -2.191323 \\ 1 & 2.961511 & 0.108960 & -2.899023 \\ 1 & 3.972838 & -1.320648 & -2.648690 \\ 1 & 2.285430 & -1.297611 & -2.086837 \\ 6 & -3.154698 & -1.093596 & -2.433322 \\ 1 & -3.361412 & -0.147697 & -2.948630 \\ 1 & -2.081984 & -1.275249 & -2.535721 \\ 1 & -3.691125 & -1.879973 & -2.982964 \\ 6 & -2.523083 & 1.462329 & 2.424812 \\ 1 & -2.963670 & 2.351204 & 1.957295 \\ 1 & -2.783568 & 1.492025 & 3.492361 \\ 1 & -1.433905 & 1.537793 & 2.344613 \\ 6 & 2.478039 & 0.199528 & 2.718280 \\ 1 & 2.782779 & 0.559740 & 3.711756 \\ 1 & 1.423786 & -0.094897 & 2.771594 \\ 1 & 3.072202 & -0.693637 & 2.490033 \\ 6 & 4.179140 & -1.303871 & 0.033649 \\ 1 & 4.887661 & -1.927509 & -0.532358 \\ 1 & 4.716884 & -0.932785 & 0.915028\end{array}$

$\begin{array}{cccc}1 & 3.353670 & -1.938695 & 0.382114 \\ 6 & -3.707639 & -2.480160 & -0.401021 \\ 1 & -4.311584 & -3.126170 & -1.054535 \\ 1 & -2.720799 & -2.946174 & -0.299066 \\ 1 & -4.180548 & -2.494008 & 0.588813 \\ 6 & -2.493068 & -1.010777 & 2.638625 \\ 1 & -2.848159 & -1.984500 & 2.277358 \\ 1 & -1.397207 & -1.012973 & 2.617269 \\ 1 & -2.814670 & -0.915154 & 3.685803 \\ 77 & -0.089984 & -0.095831 & -0.188491 \\ 6 & -0.147160 & -2.278991 & -1.041526 \\ 1 & 0.008410 & -0.840915 & -1.776616 \\ 6 & 0.045624 & -2.265649 & 0.379182 \\ 1 & -0.130993 & 0.459775 & 1.336053 \\ 1 & -0.846235 & -2.505450 & 0.975010 \\ 1 & -1.188113 & -2.375270 & -1.327314 \\ 6 & 1.575603 & -4.110387 & 0.227461 \\ 1 & 2.450839 & -4.555005 & 0.723280 \\ 1 & 0.741987 & -4.844645 & 0.316136 \\ 6 & 1.886280 & -3.837754 & -1.231279 \\ 1 & 2.133671 & -4.776958 & -1.742970 \\ 1 & 2.781277 & -3.207449 & -1.281444 \\ 6 & 0.704896 & -3.173986 & -1.935342 \\ 1 & 0.015811 & -3.971076 & -2.254395 \\ 1 & 1.019130 & -2.675930 & -2.860540 \\ 7 & 1.243771 & -2.866351 & 0.911778 \\ 6 & 1.133241 & -3.066079 & 2.336556 \\ 1 & 2.080497 & -3.441979 & 2.745016 \\ 1 & 0.899209 & -2.116754 & 2.830628 \\ 1 & 0.333812 & -3.790767 & 2.602357\end{array}$

6

The smallest/imaginary frequencies is: $37.7363 \mathrm{~cm}(-1)$

Zero-point correction=

Thermal correction to Energy=

Thermal correction to Enthalpy=

Thermal correction to Gibbs Free Energy=

Sum of electronic and zero-point Energies=

Sum of electronic and thermal Energies=

Sum of electronic and thermal Enthalpies=

Sum of electronic and thermal Free Energies=
0.797293 (Hartree/Particle)

0.845722

0.846745

0.720919

$-2017.014894$

$-2016.966465$

$-2016.965441$

$-2017.091268$

Cartesian Coordinates

$\begin{array}{cccc}6 & 0.481719 & -1.941032 & -0.762182 \\ 15 & 2.469792 & 0.050723 & 0.019539 \\ 15 & -2.120322 & -0.851965 & -0.034792 \\ 6 & 2.959941 & -1.513095 & -0.826440 \\ 6 & 3.520887 & 1.338623 & -0.935424 \\ 6 & 3.186820 & -0.174462 & 1.781794\end{array}$

$\begin{array}{lrrr}6 & -1.961046 & -2.308781 & -1.155200 \\ 6 & -2.648189 & -1.655249 & 1.621061 \\ 6 & -3.634725 & 0.039146 & -0.792995 \\ 6 & 1.792523 & -2.450552 & -0.903072 \\ 6 & 2.030954 & -3.792235 & -1.209494 \\ 6 & 0.980097 & -4.670126 & -1.439910\end{array}$ 


\begin{tabular}{|c|c|c|}
\hline-0.561352 & -2.835422 & -1.091792 \\
\hline-0.317020 & -4.172946 & -1.403965 \\
\hline 3.061976 & -4.142518 & -1.281376 \\
\hline 1.168919 & -5.717832 & -1.664991 \\
\hline-1.161245 & -4.828575 & -1.623507 \\
\hline 3.244781 & -1.232977 & -1.851049 \\
\hline 3.851949 & -1.969181 & -0.375297 \\
\hline-2.719070 & -3.081632 & -0.967470 \\
\hline-2.145849 & -1.917315 & -2.167320 \\
\hline-4.030704 & -2.307481 & 1.580304 \\
\hline-4.848375 & -1.585476 & 1.482964 \\
\hline-4.123937 & -3.049935 & 0.777907 \\
\hline-4.191669 & -2.842348 & 2.527666 \\
\hline-4.682866 & -0.905814 & -1.386714 \\
\hline-4.282741 & -1.516022 & -2.203749 \\
\hline-5.132677 & -1.576582 & -0.649109 \\
\hline-5.496506 & -0.299148 & -1.811373 \\
\hline 4.972249 & 0.918951 & -1.194564 \\
\hline 5.590549 & 0.912486 & -0.294670 \\
\hline 5.053171 & -0.064170 & -1.672682 \\
\hline 5.422915 & 1.645604 & -1.886444 \\
\hline 4.712108 & -0.123614 & 1.850538 \\
\hline 5.194601 & -0.812486 & 1.146088 \\
\hline 5.108848 & 0.884154 & 1.683657 \\
\hline 5.026264 & -0.425196 & 2.860348 \\
\hline-1.638618 & -2.757735 & 1.954637 \\
\hline-1.680524 & -3.594146 & 1.247300 \\
\hline-0.611321 & -2.379183 & 1.976974 \\
\hline-1.876444 & -3.156750 & 2.951434 \\
\hline-3.087942 & 0.905285 & -1.927367 \\
\hline-2.600809 & 0.309577 & -2.709164 \\
\hline-3.919467 & 1.456452 & -2.393985 \\
\hline-2.345595 & 1.621109 & -1.561867 \\
\hline 2.867202 & 1.517493 & -2.309188 \\
\hline 2.926834 & 0.604797 & -2.915623 \\
\hline 1.805840 & 1.775590 & -2.252699 \\
\hline 3.397973 & 2.310106 & -2.856054 \\
\hline 2.746311 & -1.554103 & 2.280046 \\
\hline 3.217116 & -2.373716 & 1.724083 \\
\hline
\end{tabular}

$\begin{array}{cccc}1 & 3.047488 & -1.658248 & 3.332361 \\ 1 & 1.659357 & -1.675794 & 2.225112 \\ 6 & -2.611634 & -0.626734 & 2.750347 \\ 1 & -2.843413 & -1.128532 & 3.701227 \\ 1 & -1.614840 & -0.177318 & 2.840938 \\ 1 & -3.348590 & 0.174586 & 2.619566 \\ 6 & -4.324321 & 0.946942 & 0.223815 \\ 1 & -5.049285 & 1.589271 & -0.298595 \\ 1 & -4.883148 & 0.386073 & 0.981085 \\ 1 & -3.619334 & 1.604590 & 0.744294 \\ 6 & 3.529327 & 2.678626 & -0.200123 \\ 1 & 4.030461 & 3.433640 & -0.823453 \\ 1 & 2.521975 & 3.052527 & 0.019146 \\ 1 & 4.081190 & 2.627075 & 0.746542 \\ 6 & 2.619721 & 0.875968 & 2.736869 \\ 1 & 2.885680 & 1.900959 & 2.447945 \\ 1 & 1.527817 & 0.798962 & 2.807355 \\ 1 & 3.032483 & 0.706576 & 3.741978 \\ 77 & 0.106926 & -0.010101 & -0.110262 \\ 6 & -0.152662 & 2.862656 & -0.405281 \\ 7 & -1.197299 & 3.577258 & -0.875875 \\ 6 & -0.086350 & 2.314069 & 0.859231 \\ 1 & 0.161376 & -0.409394 & 1.516790 \\ 1 & 0.910861 & 2.178825 & 1.263956 \\ 1 & 0.692004 & 2.798915 & -1.084460 \\ 6 & -1.190622 & 2.660458 & 1.829919 \\ 1 & -0.798821 & 2.677271 & 2.855589 \\ 1 & -1.974759 & 1.892899 & 1.828027 \\ 6 & -1.801439 & 4.014423 & 1.469665 \\ 1 & -2.661454 & 4.249346 & 2.108706 \\ 1 & -1.053100 & 4.803922 & 1.629794 \\ 6 & -2.247038 & 4.049248 & 0.015725 \\ 6 & -1.283489 & 3.973694 & -2.261065 \\ 1 & -2.280562 & 3.747480 & -2.664451 \\ 1 & -1.102424 & 5.051533 & -2.386081 \\ 1 & -0.551479 & 3.415041 & -2.853497 \\ 1 & -2.497072 & 5.078416 & -0.281923 \\ 1 & -3.159794 & 3.449167 & -0.136170 \\ 1 & 0.018569 & 0.395968 & -1.766771\end{array}$

Enamine.log

The smallest/imaginary frequencies is: $124.8533 \mathrm{~cm}(-1)$

$\begin{array}{lc}\text { Zero-point correction }= & 0.161920 \\ \text { Thermal correction to Energy= } & 0.169925 \\ \text { Thermal correction to Enthalpy= } & 0.170949 \\ \text { Thermal correction to Gibbs Free Energy= } & 0.128168 \\ \text { Sum of electronic and zero-point Energies }= & -289.613205 \\ \text { Sum of electronic and thermal Energies }= & -289.605200 \\ \text { Sum of electronic and thermal Enthalpies }= & -289.604176 \\ \text { Sum of electronic and thermal Free Energies= } & -289.646957\end{array}$ 


\begin{tabular}{crrr}
\multicolumn{4}{c}{ Cartesian Coordinates } \\
$\ldots \ldots \ldots \ldots \ldots \ldots \ldots \ldots \ldots \ldots \ldots \ldots \ldots$ \\
6 & 0.285680 & 1.199670 & -0.151965 \\
7 & 0.971012 & -0.005945 & -0.264068 \\
6 & -1.033260 & 1.311858 & 0.067142 \\
1 & -1.473652 & 2.305582 & 0.097046 \\
1 & 0.911384 & 2.082347 & -0.287534 \\
6 & -1.917160 & 0.107504 & 0.207866 \\
1 & -2.852288 & 0.250166 & -0.351996 \\
1 & -2.222685 & -0.034728 & 1.257609 \\
6 & -1.194384 & -1.136646 & -0.301226
\end{tabular}

$\begin{array}{llll}1 & -1.715809 & -2.051673 & 0.004010 \\ 1 & -1.173069 & -1.124999 & -1.399823 \\ 6 & 0.238761 & -1.170693 & 0.202977 \\ 6 & 2.366438 & 0.002353 & 0.105612 \\ 1 & 2.522797 & 0.028195 & 1.199995 \\ 1 & 2.860626 & -0.896173 & -0.284012 \\ 1 & 2.865284 & 0.875357 & -0.330088 \\ 1 & 0.759631 & -2.071015 & -0.150934 \\ 1 & 0.244245 & -1.205711 & 1.311774\end{array}$

Alkanes (Optimized with keyword int=ultrafine) Common Intermediates

$\mathrm{H} 2$

$\begin{array}{lc}\text { Zero-point correction= } & 0.010125 \\ \text { Thermal correction to Energy= } & 0.012684 \\ \text { Thermal correction to Enthalpy= } & 0.013707 \\ \text { Thermal correction to Gibbs Free Energy= } & -0.002613 \\ \text { Sum of electronic and zero-point Energies }= & -1.158906 \\ \text { Sum of electronic and thermal Energies }= & -1.156348 \\ \text { Sum of electronic and thermal Enthalpies }= & -1.155325 \\ \text { Sum of electronic and thermal Free Energies }= & -1.171644\end{array}$

Cartesian Coordinates

$\begin{array}{llllllll}1 & 0.000000 & 0.000000 & 0.371181 & 1 & 0.000000 & 0.000000 & -0.371181\end{array}$

2

The smallest/imaginary frequencies is: $28.4515 \mathrm{~cm}(-1)$

Zero-point correction=

Thermal correction to Energy=

Thermal correction to Enthalpy=

Thermal correction to Gibbs Free Energy=

Sum of electronic and zero-point Energies=

Sum of electronic and thermal Energies=

Sum of electronic and thermal Enthalpies=

Sum of electronic and thermal Free Energies=

Cartesian Coordinates
0.615586

0.654644

0.655668

0.547167

$-1726.214659$

$-1726.175601$

$-1726.174577$

$-1726.283077$

$6 \quad-0.000000-0.000000 \quad-1.552172$ 


\begin{tabular}{crrr}
15 & -1.104660 & 2.022906 & 0.185054 \\
6 & 1.671565 & -1.900286 & -1.573279 \\
6 & 2.710188 & -2.308190 & 1.155930 \\
6 & 0.000000 & -3.565504 & 0.236458 \\
6 & -1.671565 & 1.900286 & -1.573279 \\
6 & -2.710188 & 2.308190 & 1.155930 \\
6 & -0.000000 & 3.565504 & 0.236458 \\
6 & 0.787236 & -0.919415 & -2.296950 \\
6 & 0.769783 & -0.917114 & -3.691257 \\
6 & -0.000000 & -0.000000 & -4.398203 \\
6 & -0.787236 & 0.919415 & -2.296950 \\
6 & -0.769783 & 0.917114 & -3.691257 \\
1 & 1.380789 & -1.644460 & -4.228298 \\
1 & -0.000000 & -0.000000 & -5.486234 \\
1 & -1.380789 & 1.644460 & -4.228298 \\
1 & 2.702021 & -1.518026 & -1.538690 \\
1 & 1.721107 & -2.874218 & -2.081749 \\
1 & -2.702021 & 1.518026 & -1.538690 \\
1 & -1.721107 & 2.874218 & -2.081749 \\
6 & -3.670147 & 3.309534 & 0.515313 \\
1 & -3.260600 & 4.322709 & 0.463471 \\
1 & -3.968405 & 3.013072 & -0.497541 \\
1 & -4.589694 & 3.362098 & 1.116907 \\
6 & -0.733880 & 4.890664 & 0.059557 \\
1 & -1.350697 & 4.911426 & -0.848467 \\
1 & -1.372137 & 5.134312 & 0.917380 \\
1 & 0.002295 & 5.702911 & -0.032456 \\
6 & 3.670147 & -3.309534 & 0.515313 \\
1 & 3.260600 & -4.322709 & 0.463471 \\
1 & 3.968405 & -3.013072 & -0.497541 \\
1 & 4.589694 & -3.362098 & 1.116907 \\
6 & 0.733880 & -4.890664 & 0.059557 \\
1 & 1.350697 & -4.911426 & -0.848467 \\
1 & 1.372137 & -5.134312 & 0.917380 \\
& & & \\
\hline & & & \\
1 & & &
\end{tabular}

$\begin{array}{lrrr}1 & -0.002295 & -5.702911 & -0.032456 \\ 6 & -3.405015 & 0.944608 & 1.223828 \\ 1 & -3.626144 & 0.532383 & 0.230799 \\ 1 & -2.790465 & 0.202631 & 1.749687 \\ 1 & -4.359700 & 1.048970 & 1.760546 \\ 6 & 1.014081 & 3.406810 & -0.899712 \\ 1 & 0.549739 & 3.452947 & -1.892389 \\ 1 & 1.745391 & 4.226083 & -0.840615 \\ 1 & 1.559060 & 2.456881 & -0.824196 \\ 6 & 3.405015 & -0.944608 & 1.223828 \\ 1 & 3.626144 & -0.532383 & 0.230799 \\ 1 & 2.790465 & -0.202631 & 1.749687 \\ 1 & 4.359700 & -1.048970 & 1.760546 \\ 6 & -1.014081 & -3.406810 & -0.899712 \\ 1 & -0.549739 & -3.452947 & -1.892389 \\ 1 & -1.745391 & -4.226083 & -0.840615 \\ 1 & -1.559060 & -2.456881 & -0.824196 \\ 6 & -2.386914 & 2.746300 & 2.582537 \\ 1 & -3.302289 & 2.719170 & 3.191321 \\ 1 & -1.656023 & 2.078488 & 3.060147 \\ 1 & -1.998527 & 3.770912 & 2.628480 \\ 6 & 0.778474 & 3.577390 & 1.553139 \\ 1 & 0.135206 & 3.720982 & 2.428423 \\ 1 & 1.336790 & 2.640825 & 1.687292 \\ 1 & 1.504649 & 4.403583 & 1.541147 \\ 6 & 2.386914 & -2.746300 & 2.582537 \\ 1 & 3.302289 & -2.719170 & 3.191321 \\ 1 & 1.656023 & -2.078488 & 3.060147 \\ 1 & 1.998527 & -3.770912 & 2.628480 \\ 6 & -0.778474 & -3.577390 & 1.553139 \\ 1 & -0.135206 & -3.720982 & 2.428423 \\ 1 & -1.336790 & -2.640825 & 1.687292 \\ 1 & -1.504649 & -4.403583 & 1.541147 \\ 77 & 0.000000 & 0.000000 & 0.432048\end{array}$

trans-2- $\mathrm{H} 2$

The smallest/imaginary frequencies is: $33.8788 \mathrm{~cm}(-1)$

$\begin{array}{lc}\text { Zero-point correction= } & 0.632459 \\ \text { Thermal correction to Energy= } & 0.671743 \\ \text { Thermal correction to Enthalpy= } & 0.672766 \\ \text { Thermal correction to Gibbs Free Energy= } & 0.564778 \\ \text { Sum of electronic and zero-point Energies }= & -1727.384941 \\ \text { Sum of electronic and thermal Energies= } & -1727.345657 \\ \text { Sum of electronic and thermal Enthalpies }= & -1727.344634 \\ \text { Sum of electronic and thermal Free Energies= } & -1727.452622\end{array}$

Cartesian Coordinates $\begin{array}{cccccccc}6 & 0.000013 & -1.515906 & 0.000139 & 15 & -2.329654 & 0.202372 & 0.115633 \\ 15 & 2.329693 & 0.202272 & -0.115668 & 6 & 2.362723 & -1.489312 & -0.861975\end{array}$ 


\begin{tabular}{|c|c|c|c|c|c|c|c|}
\hline 6 & 3.367844 & 1.259797 & -1.309273 & 1 & -2.159952 & 0.673015 & 3.052561 \\
\hline 6 & 3.235993 & 0.023298 & 1.550320 & 1 & -1.626091 & 2.167190 & 2.281970 \\
\hline 6 & -2.362604 & -1.488979 & 0.862476 & 1 & -3.120006 & 2.151744 & 3.248523 \\
\hline 6 & -3.367647 & 1.260286 & 1.309030 & 6 & -2.615368 & -1.171871 & -2.280541 \\
\hline 6 & -3.236224 & 0.022842 & -1.550141 & 1 & -2.816873 & -2.124683 & -1.776387 \\
\hline 6 & 1.139194 & -2.238346 & -0.411288 & 1 & -3.057272 & -1.232031 & -3.285377 \\
\hline 6 & 1.130578 & -3.633331 & -0.397796 & 1 & -1.531064 & -1.062608 & -2.395588 \\
\hline 6 & -0.000095 & -4.337708 & 0.000371 & 6 & 2.511619 & 1.573589 & -2.537826 \\
\hline 6 & -1.139195 & -2.238194 & 0.411770 & 1 & 2.160515 & 0.671755 & -3.052796 \\
\hline 6 & -1.130699 & -3.633180 & 0.398473 & 1 & 1.626384 & 2.166182 & -2.282880 \\
\hline 1 & 2.028162 & -4.169778 & -0.708457 & 1 & 3.120477 & 2.150491 & -3.249153 \\
\hline 1 & -0.000144 & -5.425529 & 0.000437 & 6 & 2.615044 & -1.171161 & 2.281059 \\
\hline 1 & -2.028317 & -4.169509 & 0.709241 & 1 & 2.816633 & -2.124156 & 1.777286 \\
\hline 1 & 2.320808 & -1.325697 & -1.949714 & 1 & 3.056831 & -1.230960 & 3.285969 \\
\hline 1 & 3.290271 & -2.039866 & -0.652334 & 1 & 1.530727 & -1.061861 & 2.395939 \\
\hline 1 & -2.320437 & -1.325023 & 1.950154 & 6 & -3.732682 & 2.588353 & 0.646948 \\
\hline 1 & -3.290215 & -2.039570 & 0.653218 & 1 & -4.167288 & 3.260401 & 1.400555 \\
\hline 6 & -4.634154 & 0.554454 & 1.797416 & 1 & -2.850577 & 3.093198 & 0.228652 \\
\hline 1 & -5.338270 & 0.316099 & 0.996378 & 1 & -4.475219 & 2.475833 & -0.151081 \\
\hline 1 & -4.407105 & -0.371620 & 2.339171 & 6 & -2.998229 & 1.269597 & -2.402893 \\
\hline 1 & -5.157527 & 1.216970 & 2.501979 & 1 & -3.473803 & 2.165928 & -1.990331 \\
\hline 6 & -4.737208 & -0.231687 & -1.427794 & 1 & -1.925805 & 1.467052 & -2.521771 \\
\hline 1 & -4.968670 & -1.094659 & -0.789986 & 1 & -3.423755 & 1.106504 & -3.403518 \\
\hline 1 & -5.287220 & 0.638454 & -1.052039 & 6 & 3.732647 & 2.588141 & -0.647619 \\
\hline 1 & -5.138829 & -0.454460 & -2.426916 & 1 & 4.167345 & 3.259946 & -1.401389 \\
\hline 6 & 4.634494 & 0.553884 & -1.797174 & 1 & 2.850421 & 3.093067 & -0.229677 \\
\hline 1 & 5.338557 & 0.316009 & -0.995949 & 1 & 4.475037 & 2.475971 & 0.150597 \\
\hline 1 & 4.407624 & -0.372480 & -2.338508 & 6 & 2.997863 & 1.270345 & 2.402611 \\
\hline 1 & 5.157860 & 1.216127 & -2.501999 & 1 & 3.473421 & 2.166557 & 1.989771 \\
\hline 6 & 4.736995 & -0.231266 & 1.428286 & 1 & 1.925416 & 1.467779 & 2.521322 \\
\hline 1 & 4.968542 & -1.094456 & 0.790803 & 1 & 3.423317 & 1.107625 & 3.403326 \\
\hline 1 & 5.287054 & 0.638754 & 1.052322 & 77 & 0.000024 & 0.489549 & -0.000160 \\
\hline 1 & 5.138471 & -0.453704 & 2.427540 & 1 & -0.025469 & 0.497514 & -1.695459 \\
\hline 6 & -2.511228 & 1.574619 & 2.537307 & 1 & 0.025544 & 0.498002 & 1.695103 \\
\hline
\end{tabular}

cis-2- $\mathrm{H} 2$

The smallest/imaginary frequencies is: $22.5273 \mathrm{~cm}(-1)$

Zero-point correction=

Thermal correction to Energy=

Thermal correction to Enthalpy=

Thermal correction to Gibbs Free Energy=

Sum of electronic and zero-point Energies=

Sum of electronic and thermal Energies=

Sum of electronic and thermal Enthalpies=

Sum of electronic and thermal Free Energies=
0.628632

0.669712

0.670736

0.557141

$-1727.402315$

$-1727.361235$

$-1727.360212$

$-1727.473806$

Cartesian Coordinates

$\begin{array}{cccc}6 & 0.000015 & 1.638456 & -0.000162 \\ 15 & 2.305672 & -0.166548 & 0.068038\end{array}$
15

6 $\begin{array}{lll}-2.305651 & -0.166572 & -0.068046\end{array}$

$\begin{array}{llll}2.474973 & 1.637700 & 0.504812\end{array}$ 


$\begin{array}{lrrr}6 & 3.286137 & -1.030476 & 1.439828 \\ 6 & 3.164396 & -0.320590 & -1.615517 \\ 6 & -2.474908 & 1.637560 & -0.505278 \\ 6 & -3.286150 & -1.030830 & -1.439596 \\ 6 & -3.164391 & -0.320191 & 1.615536 \\ 6 & 1.189987 & 2.371381 & 0.215311 \\ 6 & 1.186648 & 3.768130 & 0.206470 \\ 6 & -0.000037 & 4.463547 & -0.000284 \\ 6 & -1.189980 & 2.371321 & -0.215732 \\ 6 & -1.186694 & 3.768071 & -0.207003 \\ 1 & 2.114804 & 4.315852 & 0.374506 \\ 1 & -0.000058 & 5.552265 & -0.000317 \\ 1 & -2.114868 & 4.315746 & -0.375094 \\ 1 & 2.679547 & 1.682038 & 1.584133 \\ 1 & 3.342839 & 2.106546 & 0.017880 \\ 1 & -2.679240 & 1.681581 & -1.584659 \\ 1 & -3.342891 & 2.106535 & -0.018675 \\ 6 & -4.626430 & -0.373420 & -1.770034 \\ 1 & -5.338276 & -0.412500 & -0.940438 \\ 1 & -4.517405 & 0.674472 & -2.074549 \\ 1 & -5.085068 & -0.906771 & -2.615417 \\ 6 & -4.672353 & -0.089601 & 1.579662 \\ 1 & -4.945925 & 0.854843 & 1.091645 \\ 1 & -5.204986 & -0.905999 & 1.077507 \\ 1 & -5.053649 & -0.044147 & 2.610293 \\ 6 & 4.626522 & -0.373145 & 1.769993 \\ 1 & 5.338373 & -0.412739 & 0.940426 \\ 1 & 4.517686 & 0.674910 & 2.074018 \\ 1 & 5.085053 & -0.906188 & 2.615628 \\ 6 & 4.672337 & -0.089846 & -1.579733 \\ 1 & 4.945833 & 0.854730 & -1.091927 \\ 1 & 5.205065 & -0.906080 & -1.077412 \\ 1 & 5.053605 & -0.044581 & -2.610383 \\ 6 & -2.397799 & -0.972332 & -2.687350\end{array}$

$\begin{array}{rrrr}1 & -2.147312 & 0.055995 & -2.980570 \\ 1 & -1.454201 & -1.510401 & -2.536971 \\ 1 & -2.930838 & -1.434940 & -3.530764 \\ 6 & -2.521327 & 0.731424 & 2.524668 \\ 1 & -2.726933 & 1.758516 & 2.198942 \\ 1 & -2.930265 & 0.619848 & 3.539256 \\ 1 & -1.432131 & 0.605724 & 2.581089 \\ 6 & 2.397839 & -0.971469 & 2.687597 \\ 1 & 2.147590 & 0.056984 & 2.980574 \\ 1 & 1.454117 & -1.509370 & 2.537388 \\ 1 & 2.930810 & -1.433981 & 3.531107 \\ 6 & 2.521219 & 0.730707 & -2.524939 \\ 1 & 2.726722 & 1.757916 & -2.199516 \\ 1 & 2.930160 & 0.618881 & -3.539499 \\ 1 & 1.432037 & 0.604876 & -2.581317 \\ 6 & -3.512475 & -2.497309 & -1.079101 \\ 1 & -3.890589 & -3.034245 & -1.961027 \\ 1 & -2.583523 & -2.990285 & -0.763643 \\ 1 & -4.258445 & -2.618656 & -0.283983 \\ 6 & -2.873644 & -1.695627 & 2.217029 \\ 1 & -3.302441 & -2.516091 & 1.631180 \\ 1 & -1.794434 & -1.870685 & 2.305157 \\ 1 & -3.311626 & -1.747409 & 3.224553 \\ 6 & 3.512249 & -2.497104 & 1.079795 \\ 1 & 3.890329 & -3.033809 & 1.961876 \\ 1 & 2.583216 & -2.990051 & 0.764530 \\ 1 & 4.258169 & -2.618808 & 0.284685 \\ 6 & 2.873748 & -1.696209 & -2.216635 \\ 1 & 3.302614 & -2.516487 & -1.630579 \\ 1 & 1.794548 & -1.871367 & -2.304700 \\ 1 & 3.311719 & -1.748223 & -3.224150 \\ 77 & 0.000008 & -0.481016 & 0.000036 \\ 1 & 0.001481 & -1.920307 & -0.730724 \\ 1 & -0.001444 & -1.919977 & 0.731282\end{array}$

Geometries from ( $\left.{ }^{(} \mathrm{Pr}\right)_{2} \mathrm{C}(\mathrm{H})\left(\mathrm{CH}_{2} \mathrm{CH}_{3}\right)$ dehydrogenation

1

The smallest/imaginary frequencies is: $91.2621 \mathrm{~cm}(-1)$ 


$\begin{array}{lc}\text { Zero-point correction= } & 0.272706 \\ \text { Thermal correction to Energy= } & 0.286375 \\ \text { Thermal correction to Enthalpy= } & 0.287399 \\ \text { Thermal correction to Gibbs Free Energy= } & 0.232716 \\ \text { Sum of electronic and zero-point Energies }= & -354.461817 \\ \text { Sum of electronic and thermal Energies }= & -354.448147 \\ \text { Sum of electronic and thermal Enthalpies }= & -354.447124 \\ \text { Sum of electronic and thermal Free Energies }= & -354.501807\end{array}$

Cartesian Coordinates

$\begin{array}{rrrrrrrrr}6 & -1.263452 & -2.207999 & 0.425442 & 1 & 2.393255 & 1.336706 & -0.634230 \\ 1 & -1.760519 & -1.628268 & 1.214343 & 1 & 3.415846 & -0.103815 & -0.661177 \\ 1 & -0.349578 & -2.635619 & 0.856286 & 6 & -0.779538 & 0.934157 & 0.323811 \\ 1 & -1.922947 & -3.048121 & 0.177856 & 6 & -2.230492 & 1.182484 & -0.079719 \\ 6 & -0.968753 & -1.367899 & -0.808092 & 6 & -0.042644 & 2.270661 & 0.297205 \\ 1 & -0.456870 & -1.997524 & -1.551356 & 1 & -0.779031 & 0.565292 & 1.362701 \\ 1 & -1.915477 & -1.075517 & -1.281937 & 1 & -2.875006 & 0.310108 & 0.075066 \\ 6 & 1.340760 & -0.526986 & -0.165268 & 1 & -2.656941 & 2.007978 & 0.503846 \\ 6 & 1.621416 & -0.522998 & 1.335747 & 1 & -2.298217 & 1.464622 & -1.140914 \\ 6 & 2.412766 & 0.272659 & -0.901111 & 1 & 0.982381 & 2.208268 & 0.677084 \\ 1 & 1.463040 & -1.571872 & -0.498194 & 1 & 0.008136 & 2.663285 & -0.729147 \\ 1 & 0.887244 & -1.102462 & 1.907510 & 1 & -0.567854 & 3.017298 & 0.906510 \\ 1 & 2.608123 & -0.961552 & 1.532941 & 6 & -0.103032 & -0.118751 & -0.579452 \\ 1 & 1.636048 & 0.491910 & 1.753358 & 1 & -0.020011 & 0.361710 & -1.572797 \\ 1 & 2.286192 & 0.205600 & -1.989121 & & & & \end{array}$

4

The smallest/imaginary frequencies is: $26.4002 \mathrm{~cm}(-1)$

Zero-point correction=

Thermal correction to Energy=

Thermal correction to Enthalpy=

Thermal correction to Gibbs Free Energy=

Sum of electronic and zero-point Energies=

Sum of electronic and thermal Energies=

Sum of electronic and thermal Enthalpies=

Sum of electronic and thermal Free Energies=
0.891624

0.945693

0.946716

0.807410

$-2080.683819$

$-2080.629750$

$-2080.628727$

$-2080.768033$

Cartesian Coordinates

$\begin{array}{cccc}6 & 2.585241 & 0.290973 & -0.260850 \\ 15 & 0.605704 & 2.381893 & -0.057662 \\ 15 & 1.196843 & -2.194148 & 0.142559 \\ 6 & 2.194141 & 2.688253 & -0.953615 \\ 6 & -0.679171 & 3.182013 & -1.208752 \\ 6 & 0.763019 & 3.363314 & 1.558497 \\ 6 & 3.029083 & -2.111226 & 0.379970 \\ 6 & 0.632491 & -3.319421 & 1.563487 \\ 6 & 0.966834 & -3.064367 & -1.530421 \\ 6 & 3.118033 & 1.527648 & -0.714192\end{array}$

$\begin{array}{lrrr}6 & 4.479836 & 1.681539 & -0.972891 \\ 6 & 5.363927 & 0.619689 & -0.821604 \\ 6 & 3.512639 & -0.775323 & -0.110485 \\ 6 & 4.867216 & -0.607094 & -0.396910 \\ 1 & 4.845904 & 2.652551 & -1.310572 \\ 1 & 6.423595 & 0.745817 & -1.033842 \\ 1 & 5.541870 & -1.455947 & -0.274239 \\ 1 & 1.938129 & 2.739640 & -2.021701 \\ 1 & 2.653943 & 3.654150 & -0.699065 \\ 1 & 3.197699 & -2.194033 & 1.463475\end{array}$




\begin{tabular}{lrrr}
1 & 3.568501 & -2.949407 & -0.084994 \\
6 & 1.554194 & -4.506681 & 1.840561 \\
1 & 1.630734 & -5.196086 & 0.994002 \\
1 & 2.568238 & -4.192749 & 2.115065 \\
1 & 1.154759 & -5.079099 & 2.690977 \\
6 & 1.387286 & -4.530900 & -1.550252 \\
1 & 2.420695 & -4.675540 & -1.209001 \\
1 & 0.731031 & -5.165790 & -0.943355 \\
1 & 1.332788 & -4.908025 & -2.582196 \\
6 & -0.252228 & 4.522332 & -1.806830 \\
1 & -0.095723 & 5.297656 & -1.050482 \\
1 & 0.662744 & 4.442850 & -2.405574 \\
1 & -1.045127 & 4.880746 & -2.480096 \\
6 & 0.761152 & 4.879867 & 1.396569 \\
1 & 1.525679 & 5.226838 & 0.689156 \\
1 & -0.212022 & 5.265775 & 1.070435 \\
1 & 0.983245 & 5.347872 & 2.367115 \\
6 & 0.608528 & -2.424904 & 2.806752 \\
1 & 1.594582 & -2.000195 & 3.036228 \\
1 & -0.083188 & -1.580891 & 2.686375 \\
1 & 0.289196 & -3.017296 & 3.677298 \\
6 & 1.818573 & -2.296640 & -2.545046 \\
1 & 2.892846 & -2.363542 & -2.333512 \\
1 & 1.650823 & -2.724949 & -3.544073 \\
1 & 1.546371 & -1.233680 & -2.577350 \\
6 & -0.888211 & 2.185371 & -2.353565 \\
1 & 0.033307 & 1.996632 & -2.920245 \\
1 & -1.240695 & 1.212596 & -1.985516 \\
1 & -1.636131 & 2.586371 & -3.054405 \\
6 & 2.089860 & 2.935347 & 2.191180 \\
1 & 2.960012 & 3.252338 & 1.603170 \\
1 & 2.176626 & 3.399094 & 3.184662 \\
1 & 2.144310 & 1.846032 & 2.315599 \\
6 & -0.784281 & -3.826140 & 1.304676 \\
1 & -1.201148 & -4.246643 & 2.232015 \\
1 & -1.454118 & -3.019105 & 0.979698 \\
1 & -0.811721 & -4.618771 & 0.547146 \\
6 & -0.494124 & -2.937606 & -1.964326 \\
1 & -1.180027 & -3.503895 & -1.323388 \\
1 & -0.811352 & -1.885114 & -1.963244 \\
\hline & &
\end{tabular}

$\begin{array}{lrrr}1 & -0.608500 & -3.323560 & -2.988366 \\ 6 & -2.006608 & 3.356159 & -0.476059 \\ 1 & -2.803882 & 3.581079 & -1.201227 \\ 1 & -2.295835 & 2.443088 & 0.058652 \\ 1 & -1.981406 & 4.182216 & 0.245310 \\ 6 & -0.361339 & 2.942522 & 2.506688 \\ 1 & -1.354442 & 3.232862 & 2.143729 \\ 1 & -0.355601 & 1.854681 & 2.665565 \\ 1 & -0.215221 & 3.427158 & 3.483401 \\ 77 & 0.645557 & 0.065115 & 0.158509 \\ 6 & -2.727786 & -0.149231 & 1.083487 \\ 1 & -2.611721 & 0.867106 & 1.491712 \\ 6 & -4.845527 & 0.875435 & 0.035173 \\ 1 & -4.368472 & 1.837590 & 0.292591 \\ 6 & -3.883974 & -1.501391 & -0.749647 \\ 1 & -2.991718 & -2.084007 & -0.455403 \\ 6 & -5.823179 & 0.592652 & 1.173293 \\ 1 & -5.331497 & 0.595542 & 2.152155 \\ 1 & -6.588409 & 1.379751 & 1.201677 \\ 6 & -5.605048 & 1.120167 & -1.264303 \\ 1 & -6.297103 & 1.964564 & -1.150260 \\ 1 & -4.922932 & 1.358979 & -2.092070 \\ 1 & -6.206927 & 0.251759 & -1.562651 \\ 6 & -5.099798 & -2.268804 & -0.237533 \\ 1 & -6.037606 & -1.825612 & -0.597442 \\ 1 & -5.073492 & -3.301304 & -0.610481 \\ 1 & -5.150795 & -2.316292 & 0.855795 \\ 6 & -3.889726 & -1.510003 & -2.277460 \\ 1 & -4.790132 & -1.040131 & -2.691724 \\ 1 & -3.019353 & -0.981999 & -2.690368 \\ 1 & -3.860757 & -2.540914 & -2.655859 \\ 6 & -3.076066 & -1.096342 & 2.223874 \\ 1 & -2.295029 & -1.060812 & 2.994955 \\ 1 & -4.023027 & -0.840965 & 2.711719 \\ 1 & -3.151299 & -2.138439 & 1.891911 \\ 1 & -1.720814 & -0.441444 & 0.704213 \\ 1 & -6.348592 & -0.362025 & 1.060733 \\ 6 & -3.648994 & -0.089673 & -0.150340 \\ 1 & -3.033872 & 0.429723 & -0.907215\end{array}$

TS1

The smallest/imaginary frequencies is: $-581.0097 \mathrm{~cm}(-1)$

$\begin{array}{lc}\text { Zero-point correction }= & 0.884989 \\ \text { Thermal correction to Energy= } & 0.939051 \\ \text { Thermal correction to Enthalpy= } & 0.940074 \\ \text { Thermal correction to Gibbs Free Energy }= & 0.799609 \\ \text { Sum of electronic and zero-point Energies }= & -2080.663119 \\ \text { Sum of electronic and thermal Energies }= & -2080.609057 \\ \text { Sum of electronic and thermal Enthalpies }= & -2080.608034\end{array}$


Cartesian Coordinates

\begin{tabular}{|c|c|c|c|c|c|c|c|}
\hline 6 & -2.399501 & -0.105016 & -0.924747 & 6 & -2.569227 & -2.514609 & 2.053513 \\
\hline 15 & -0.759695 & -2.350520 & -0.017301 & 1 & -3.397557 & -2.793287 & 1.390854 \\
\hline 15 & -1.040512 & 2.277601 & 0.099481 & 1 & -2.829120 & -2.858314 & 3.065241 \\
\hline 6 & -2.258268 & -2.621149 & -1.078204 & 1 & -2.505131 & -1.419264 & 2.069482 \\
\hline 6 & 0.546965 & -3.392473 & -0.927462 & 6 & -0.583768 & 2.637167 & 2.856621 \\
\hline 6 & -1.239141 & -3.150107 & 1.639137 & 1 & -0.956090 & 2.988649 & 3.829990 \\
\hline 6 & -2.545160 & 2.416083 & -0.977114 & 1 & -0.381935 & 1.562770 & 2.946156 \\
\hline 6 & -1.637139 & 2.920334 & 1.785300 & 1 & 0.367017 & 3.149568 & 2.667982 \\
\hline 6 & 0.137122 & 3.522978 & -0.729544 & 6 & 1.362685 & 3.753452 & 0.153966 \\
\hline 6 & -3.029900 & -1.338962 & -1.223320 & 1 & 2.106947 & 4.345173 & -0.399339 \\
\hline 6 & -4.349037 & -1.401544 & -1.677167 & 1 & 1.110606 & 4.324362 & 1.056573 \\
\hline 6 & -5.083228 & -0.238791 & -1.876177 & 1 & 1.844342 & 2.818924 & 0.465920 \\
\hline 6 & -3.164861 & 1.060916 & -1.179271 & 6 & 1.804516 & -3.531663 & -0.072260 \\
\hline 6 & -4.483510 & 0.990971 & -1.633455 & 1 & 2.606207 & -3.989268 & -0.670480 \\
\hline 1 & -4.802896 & -2.373331 & -1.877275 & 1 & 2.171530 & -2.563707 & 0.291705 \\
\hline 1 & -6.114528 & -0.290299 & -2.220411 & 1 & 1.640608 & -4.184378 & 0.794193 \\
\hline 1 & -5.045309 & 1.911582 & -1.798126 & 6 & -0.202807 & -2.804866 & 2.708935 \\
\hline 1 & -1.903912 & -2.941087 & -2.068142 & 1 & 0.796286 & -3.192174 & 2.477644 \\
\hline 1 & -2.882046 & -3.446044 & -0.705751 & 1 & -0.124225 & -1.720743 & 2.855859 \\
\hline 1 & -3.263452 & 3.151806 & -0.587903 & 1 & -0.512281 & -3.249144 & 3.666120 \\
\hline 1 & -2.212489 & 2.809515 & -1.947887 & 77 & -0.552395 & -0.020079 & 0.0743 \\
\hline 6 & -1.987248 & 4.405529 & 1.809809 & 6 & 2.505830 & 0.263571 & -0.139282 \\
\hline 1 & -1.096981 & 5.043192 & 1.759284 & 1 & 2.176906 & 1.108979 & -0.752583 \\
\hline 1 & -2.668398 & 4.696141 & 0.999961 & 6 & 4.658524 & 1.402744 & -0.805483 \\
\hline 1 & -2.493354 & 4.639367 & 2.757870 & 1 & 4.060899 & 2.332554 & -0.780687 \\
\hline 6 & -0.474419 & 4.883333 & -1.071086 & 6 & 4.653365 & -0.811585 & 0.693555 \\
\hline 1 & -1.406678 & 4.802144 & -1.642608 & 1 & 3.867631 & -1.354703 & 1.247998 \\
\hline 1 & -0.670175 & 5.498181 & -0.188318 & 6 & 4.584901 & 0.906637 & -2.249060 \\
\hline 1 & 0.237653 & 5.439810 & -1.698610 & 1 & 4.898942 & 1.707333 & -2.931776 \\
\hline 6 & 0.088339 & -4.787429 & -1.356075 & 1 & 5.247340 & 0.053163 & -2.433453 \\
\hline 1 & -0.051381 & -5.468928 & -0.512405 & 6 & 6.089850 & 1.802203 & -0.468781 \\
\hline 1 & -0.840572 & -4.774405 & -1.938218 & 1 & 6.431325 & 2.604000 & -1.136789 \\
\hline 1 & 0.861878 & -5.229911 & -2.001091 & 1 & 6.183305 & 2.168101 & 0.561940 \\
\hline 6 & -1.414583 & -4.665484 & 1.586616 & 1 & 6.789849 & 0.965918 & -0.594638 \\
\hline 1 & -2.080532 & -4.991451 & 0.777506 & 6 & 5.089890 & -1.726969 & -0.446201 \\
\hline 1 & -0.458467 & -5.191298 & 1.480817 & 1 & 5.988732 & -1.347411 & -0.950066 \\
\hline 1 & -1.863217 & -5.006560 & 2.531124 & 1 & 5.340464 & -2.723166 & -0.057242 \\
\hline 6 & -2.891127 & 2.113726 & 2.134944 & 1 & 4.309472 & -1.860421 & -1.206984 \\
\hline 1 & -3.735012 & 2.331804 & 1.469001 & 6 & 5.799839 & -0.633575 & 1.685054 \\
\hline 1 & -2.700795 & 1.033858 & 2.095007 & 1 & 5.510936 & 0.010847 & 2.525412 \\
\hline 1 & -3.205249 & 2.369191 & 3.157361 & 1 & 6.099800 & -1.604114 & 2.102076 \\
\hline 6 & 0.559889 & 2.865105 & -2.051787 & 1 & 6.692093 & -0.195523 & 1.222409 \\
\hline 1 & -0.236533 & 2.919079 & -2.805031 & 6 & 1.547201 & 0.095149 & 1.039546 \\
\hline 1 & 1.433408 & 3.392302 & -2.463895 & 1 & 0.081350 & -0.021967 & 1.538307 \\
\hline 1 & 0.819803 & 1.805750 & -1.938304 & 1 & 1.836485 & -0.793302 & 1.621274 \\
\hline 6 & 0.884336 & -2.598079 & -2.195003 & 1 & 1.699476 & 0.947575 & 1.721516 \\
\hline 1 & 0.033813 & -2.538771 & -2.886774 & 1 & 3.570145 & 0.611449 & -2.545164 \\
\hline 1 & 1.190263 & -1.568603 & -1.973259 & 1 & 2.454679 & -0.616029 & -0.794967 \\
\hline 1 & 1.706216 & -3.096168 & -2.730263 & 6 & 3.976192 & 0.509819 & 0.26095 \\
\hline
\end{tabular}


5

The smallest/imaginary frequencies is: $23.5152 \mathrm{~cm}(-1)$

$\begin{array}{lc}\text { Zero-point correction }= & 0.887452 \\ \text { Thermal correction to Energy= } & 0.941160 \\ \text { Thermal correction to Enthalpy= } & 0.942184 \\ \text { Thermal correction to Gibbs Free Energy= } & 0.804009 \\ \text { Sum of electronic and zero-point Energies= } & -2080.670672 \\ \text { Sum of electronic and thermal Energies= } & -2080.616964 \\ \text { Sum of electronic and thermal Enthalpies= } & -2080.615940 \\ \text { Sum of electronic and thermal Free Energies= } & -2080.754115\end{array}$

Cartesian Coordinates

$\begin{array}{rrrr}1 & -0.090398 & 5.134966 & 1.289199 \\ 1 & 1.139453 & 5.124486 & 2.555614 \\ 6 & 2.761553 & -2.120189 & 2.322289 \\ 1 & 3.681453 & -2.313830 & 1.757850 \\ 1 & 2.585109 & -1.037902 & 2.313700 \\ 1 & 2.946609 & -2.420950 & 3.363351 \\ 6 & -0.197545 & -2.732351 & -2.245916 \\ 1 & 0.694999 & -2.500785 & -2.842102 \\ 1 & -0.852583 & -3.358681 & -2.868592 \\ 1 & -0.726146 & -1.789407 & -2.054211 \\ 6 & -0.707515 & 2.371746 & -2.386905 \\ 1 & 0.232183 & 2.258869 & -2.944148 \\ 1 & -1.062205 & 1.366770 & -2.118204 \\ 1 & -1.445314 & 2.810900 & -3.073977 \\ 6 & 2.195449 & 2.721237 & 2.240472 \\ 1 & 3.067899 & 3.079190 & 1.681109 \\ 1 & 2.279068 & 3.113663 & 3.263906 \\ 1 & 2.258224 & 1.627627 & 2.296008 \\ 6 & 0.377419 & -2.664451 & 2.725481 \\ 1 & 0.634929 & -3.025164 & 3.731957 \\ 1 & 0.134808 & -1.597628 & 2.800387 \\ 1 & -0.529101 & -3.193828 & 2.409588 \\ 6 & -1.149329 & -3.813219 & -0.206105 \\ 1 & -1.831973 & -4.348998 & -0.882271 \\ 1 & -0.966248 & -4.469938 & 0.653540 \\ 1 & -1.670576 & -2.917551 & 0.153352 \\ 6 & -1.902740 & 3.381901 & -0.448379 \\ 1 & -2.664245 & 3.722123 & -1.165702 \\ 1 & -2.233045 & 2.419610 & -0.039528 \\ 1 & -1.881776 & 4.113403 & 0.368962 \\ 6 & -0.259262 & 2.751311 & 2.571106 \\ 1 & -1.251768 & 3.042106 & 2.206974 \\ 1 & -0.259364 & 1.664294 & 2.716056 \\ 1 & -0.118897 & 3.224095 & 3.554020 \\ 77 & 0.564434 & 0.017189 & -0.025467 \\ & & & \\ 1 & & & \end{array}$




$\begin{array}{lrrr}6 & -2.609008 & -0.370908 & -0.320322 \\ 1 & -2.361583 & -1.216285 & -0.983834 \\ 6 & -4.855819 & -1.494896 & -0.720891 \\ 1 & -4.259693 & -2.421187 & -0.816029 \\ 6 & -4.627050 & 0.680336 & 0.811161 \\ 1 & -3.765562 & 1.210140 & 1.253892 \\ 6 & -5.012585 & -0.954813 & -2.141259 \\ 1 & -5.418300 & -1.738245 & -2.795231 \\ 1 & -5.706572 & -0.107945 & -2.190786 \\ 6 & -6.216381 & -1.914576 & -0.177468 \\ 1 & -6.655547 & -2.700860 & -0.806079 \\ 1 & -6.146773 & -2.307241 & 0.845313 \\ 1 & -6.929320 & -1.079520 & -0.169587 \\ 6 & -5.230138 & 1.621984 & -0.226930 \\ 1 & -6.208619 & 1.267505 & -0.578349\end{array}$

$\begin{array}{lrrr}1 & -5.389959 & 2.617048 & 0.210717 \\ 1 & -4.585680 & 1.751100 & -1.105682 \\ 6 & -5.610281 & 0.476880 & 1.960743 \\ 1 & -5.193117 & -0.181179 & 2.734012 \\ 1 & -5.847246 & 1.437613 & 2.437608 \\ 1 & -6.560513 & 0.040897 & 1.629943 \\ 6 & -1.501789 & -0.197961 & 0.725036 \\ 1 & 0.967469 & 0.092414 & 1.457782 \\ 1 & -1.762016 & 0.661081 & 1.365126 \\ 1 & -1.565750 & -1.067917 & 1.402326 \\ 1 & -4.058787 & -0.631054 & -2.574872 \\ 1 & -2.652529 & 0.508546 & -0.986588 \\ 6 & -4.020614 & -0.628670 & 0.252235 \\ 1 & -3.867887 & -1.276968 & 1.136560\end{array}$

TS2

The smallest/imaginary frequencies is: $-785.4928 \mathrm{~cm}(-1)$

$\begin{array}{lc}\text { Zero-point correction }= & 0.884172 \\ \text { Thermal correction to Energy= } & 0.936556 \\ \text { Thermal correction to Enthalpy= } & 0.937579 \\ \text { Thermal correction to Gibbs Free Energy= } & 0.804032 \\ \text { Sum of electronic and zero-point Energies }= & -2080.643466 \\ \text { Sum of electronic and thermal Energies }= & -2080.591082 \\ \text { Sum of electronic and thermal Enthalpies }= & -2080.590058 \\ \text { Sum of electronic and thermal Free Energies }= & -2080.723606\end{array}$

Cartesian Coordinates

$\begin{array}{cccc}6 & 0.381716 & -2.156966 & -0.698684 \\ 15 & 2.412389 & -0.380930 & 0.273676 \\ 15 & -2.197157 & -1.082818 & 0.041702 \\ 6 & 2.681919 & -2.205247 & 0.268766 \\ 6 & 3.255564 & 0.163641 & -1.368973 \\ 6 & 3.442798 & 0.231357 & 1.756872 \\ 6 & -2.027593 & -2.287251 & -1.357981 \\ 6 & -2.856223 & -2.113722 & 1.504314 \\ 6 & -3.580912 & 0.040251 & -0.651106 \\ 6 & 1.632456 & -2.812647 & -0.617428 \\ 6 & 1.879145 & -4.017707 & -1.274762 \\ 6 & 0.867504 & -4.647951 & -1.993254 \\ 6 & -0.640169 & -2.856849 & -1.369784 \\ 6 & -0.397887 & -4.074376 & -2.012160 \\ 1 & 2.867362 & -4.475246 & -1.204278 \\ 1 & 1.057088 & -5.587432 & -2.508669 \\ 1 & -1.215781 & -4.577445 & -2.530564 \\ 1 & 3.703356 & -2.501859 & 0.002610 \\ 1 & 2.506466 & -2.534562 & 1.302976 \\ 1 & -2.798353 & -3.070709 & -1.319416 \\ 1 & -2.211168 & -1.711903 & -2.276544\end{array}$

$\begin{array}{lrrr}6 & -4.305596 & -2.574273 & 1.367262 \\ 1 & -5.019971 & -1.751340 & 1.479105 \\ 1 & -4.504848 & -3.087365 & 0.418251 \\ 1 & -4.521165 & -3.291594 & 2.172270 \\ 6 & -4.840335 & -0.688428 & -1.133331 \\ 1 & -4.614783 & -1.535530 & -1.792364 \\ 1 & -5.477463 & -1.044411 & -0.321195 \\ 1 & -5.440889 & 0.021132 & -1.721613 \\ 6 & 4.510985 & -0.656326 & -1.681256 \\ 1 & 5.261182 & -0.642239 & -0.884718 \\ 1 & 4.268041 & -1.700833 & -1.910283 \\ 1 & 4.985376 & -0.236900 & -2.580275 \\ 6 & 4.938122 & -0.054785 & 1.628655 \\ 1 & 5.148157 & -1.112110 & 1.423455 \\ 1 & 5.419228 & 0.552290 & 0.853225 \\ 1 & 5.431337 & 0.192814 & 2.580222 \\ 6 & -1.985310 & -3.367819 & 1.625378 \\ 1 & -2.117799 & -4.051208 & 0.777519 \\ 1 & -0.919678 & -3.129750 & 1.709673 \\ 1 & -2.282803 & -3.910851 & 2.534044 \\ 6 & -3.000800 & 0.740100 & -1.890556\end{array}$




\begin{tabular}{|c|c|c|c|c|c|c|c|}
\hline 1 & -3.083251 & 0.107879 & -2.782878 & 1 & -0.015166 & 0.998436 & -0.812476 \\
\hline 1 & -3.581256 & 1.652225 & -2.093630 & 6 & -0.249011 & 3.500876 & -1.644033 \\
\hline 1 & -1.944960 & 1.011426 & -1.785947 & 1 & 0.131003 & 2.558093 & -2.076400 \\
\hline 6 & 2.284582 & -0.051276 & -2.533700 & 6 & -1.267236 & 4.162273 & 0.735767 \\
\hline 1 & 1.981142 & -1.098780 & -2.635946 & 1 & -1.785556 & 3.599283 & 1.528654 \\
\hline 1 & 1.372718 & 0.545872 & -2.430817 & 6 & 0.946320 & 4.445897 & -1.542922 \\
\hline 1 & 2.791905 & 0.246012 & -3.463371 & 1 & 1.504128 & 4.450438 & -2.488742 \\
\hline 6 & 2.919912 & -0.499757 & 2.997578 & 1 & 0.630843 & 5.480644 & -1.355000 \\
\hline 1 & 3.201368 & -1.559112 & 3.009025 & 6 & -1.243200 & 4.046623 & -2.665672 \\
\hline 1 & 3.363337 & -0.040069 & 3.892311 & 1 & -0.767402 & 4.122014 & -3.652140 \\
\hline 1 & 1.828459 & -0.438504 & 3.086690 & 1 & -2.119533 & 3.395053 & -2.773719 \\
\hline 6 & -2.717785 & -1.297754 & 2.789986 & 1 & -1.602419 & 5.049714 & -2.408512 \\
\hline 1 & -3.061259 & -1.904842 & 3.640279 & 6 & -0.117526 & 4.888572 & 1.429365 \\
\hline 1 & -1.676225 & -1.010315 & 2.972708 & 1 & 0.381409 & 5.609616 & 0.772485 \\
\hline 1 & -3.326500 & -0.384712 & 2.775643 & 1 & -0.503953 & 5.453562 & 2.287436 \\
\hline 6 & -3.992198 & 1.072122 & 0.398823 & 1 & 0.643428 & 4.198328 & 1.814553 \\
\hline 1 & -4.694964 & 1.792702 & -0.045796 & 6 & -2.279389 & 5.168343 & 0.202135 \\
\hline 1 & -4.505032 & 0.605559 & 1.249392 & 1 & -3.114602 & 4.673031 & -0.312830 \\
\hline 1 & -3.141839 & 1.640782 & 0.792195 & 1 & -2.700785 & 5.755034 & 1.028746 \\
\hline 6 & 3.624791 & 1.645676 & -1.339126 & 1 & -1.828474 & 5.883742 & -0.497160 \\
\hline 1 & 3.946397 & 1.953618 & -2.344626 & 6 & -0.366453 & 1.399144 & 1.634705 \\
\hline 1 & 2.772212 & 2.280985 & -1.068448 & 1 & 0.148902 & -1.364163 & 1.611090 \\
\hline 1 & 4.454777 & 1.863990 & -0.657292 & 1 & 0.340261 & 1.436074 & 2.462802 \\
\hline 6 & 3.240142 & 1.731107 & 1.991857 & 1 & -1.402846 & 1.483566 & 1.965405 \\
\hline 1 & 3.560755 & 2.354671 & 1.151494 & 1 & 1.652925 & 4.168095 & -0.749933 \\
\hline 1 & 2.195898 & 1.971322 & 2.218134 & 1 & 1.049657 & 2.294354 & 0.327575 \\
\hline 1 & 3.835688 & 2.034951 & 2.864742 & 6 & -0.882579 & 3.071423 & -0.299084 \\
\hline 77 & 0.039887 & -0.339067 & 0.346843 & 1 & -1.840846 & 2.599382 & -0.55440 \\
\hline 6 & -0.007919 & 2.019305 & 0.391973 & & & & \\
\hline
\end{tabular}

TS1'

The smallest/imaginary frequencies is: $-635.2292 \mathrm{~cm}(-1)$

Zero-point correction=

Thermal correction to Energy=

Thermal correction to Enthalpy=

Thermal correction to Gibbs Free Energy=

Sum of electronic and zero-point Energies=

Sum of electronic and thermal Energies=

Sum of electronic and thermal Enthalpies=

Sum of electronic and thermal Free Energies=
0.885952

0.939679

0.940702

0.802522

$-2080.649820$

$-2080.596093$

$-2080.595070$

$-2080.733250$

Cartesian Coordinates

$\begin{array}{cccc}6 & -0.903882 & -2.287488 & 0.072309 \\ 15 & -2.436091 & 0.211570 & 0.099314 \\ 15 & 1.897761 & -1.535438 & -0.069917 \\ 6 & -3.131348 & -1.365112 & 0.776512 \\ 6 & -3.051353 & 1.474550 & 1.389817 \\ 6 & -3.404613 & 0.442710 & -1.528101 \\ 6 & 1.363020 & -3.287960 & -0.372210\end{array}$

$\begin{array}{lrrr}6 & 3.281424 & -1.402140 & -1.386978 \\ 6 & 2.643878 & -1.607478 & 1.682297 \\ 6 & -2.259797 & -2.526210 & 0.404900 \\ 6 & -2.790225 & -3.814753 & 0.481264 \\ 6 & -1.979015 & -4.923641 & 0.269717 \\ 6 & -0.102608 & -3.440279 & -0.095318 \\ 6 & -0.630641 & -4.730859 & 0.001486\end{array}$




$$
\begin{array}{rrr}
-3.843951 & -3.948970 & 0.729619 \\
-2.391582 & -5.928989 & 0.331519 \\
0.025671 & -5.590820 & -0.140073 \\
-3.107205 & -1.252269 & 1.870103 \\
-4.186016 & -1.518679 & 0.508703 \\
1.570667 & -3.517216 & -1.425738 \\
1.963398 & -3.999378 & 0.213555 \\
4.277535 & -2.565503 & -1.398415 \\
4.969992 & -2.546474 & -0.553017 \\
3.792930 & -3.548403 & -1.421476 \\
4.886144 & -2.488766 & -2.311629 \\
3.894779 & -2.471304 & 1.821713 \\
3.775930 & -3.477071 & 1.399864 \\
4.774557 & -2.004326 & 1.364762 \\
4.120890 & -2.595043 & 2.890852 \\
-4.437599 & 1.154505 & 1.957385 \\
-5.217961 & 1.102468 & 1.192268 \\
-4.455979 & 0.218439 & 2.526229 \\
-4.720463 & 1.956033 & 2.655396 \\
-4.915870 & 0.579354 & -1.353363 \\
-5.359505 & -0.244727 & -0.781449 \\
-5.199405 & 1.525006 & -0.876922 \\
-5.388400 & 0.569104 & -2.346627 \\
2.572241 & -1.404758 & -2.747505 \\
2.184287 & -2.396576 & -3.009707 \\
1.736261 & -0.701494 & -2.792819 \\
3.293286 & -1.127192 & -3.529884 \\
1.556689 & -2.172237 & 2.601585 \\
1.314434 & -3.218464 & 2.376900 \\
1.917989 & -2.132638 & 3.639653 \\
0.629229 & -1.588816 & 2.535928 \\
-2.049700 & 1.429540 & 2.545883 \\
-1.935812 & 0.418748 & 2.960788 \\
-1.055208 & 1.767689 & 2.236953 \\
-2.400040 & 2.086964 & 3.355496 \\
-3.123580 & -0.806778 & -2.368189 \\
-3.518435 & -1.721778 & -1.909768 \\
-3.606093 & -0.692595 & -3.349763 \\
-2.048868 & -0.956693 & -2.534323 \\
4.066174 & -0.102741 & -1.232930 \\
4.780600 & -0.006928 & -2.063568 \\
3.419230 & 0.781669 & -1.251800 \\
4.649278 & -0.079122 & -0.303792
\end{array}
$$

\begin{tabular}{llll}
6 & 2.967650 & -0.192219 & 2.154427 \\
1 & 3.373646 & -0.230296 & 3.176163 \\
1 & 3.716229 & 0.301659 & 1.522657 \\
1 & 2.067989 & 0.433436 & 2.172609 \\
6 & -3.086777 & 2.890701 & 0.816939 \\
1 & -3.193267 & 3.612252 & 1.640523 \\
1 & -2.172428 & 3.150176 & 0.270469 \\
1 & -3.939148 & 3.041684 & 0.143816 \\
6 & -2.894176 & 1.657030 & -2.305145 \\
1 & -3.096971 & 2.606370 & -1.798216 \\
1 & -1.819102 & 1.596949 & -2.500690 \\
1 & -3.403882 & 1.694263 & -3.279064 \\
77 & -0.140077 & -0.322286 & -0.119049 \\
6 & 0.458579 & 1.844172 & -0.741643 \\
1 & -0.492338 & 2.381900 & -0.627034 \\
6 & 1.351681 & 3.082809 & 1.366589 \\
1 & 0.915115 & 2.168529 & 1.802365 \\
6 & 1.884036 & 3.948619 & -1.068365 \\
1 & 2.212785 & 3.486659 & -2.011472 \\
6 & 0.368356 & 4.207420 & 1.679101 \\
1 & 0.101305 & 4.187114 & 2.744963 \\
1 & 0.803563 & 5.195578 & 1.480995 \\
6 & 2.640202 & 3.315182 & 2.157962 \\
1 & 2.439496 & 3.235663 & 3.235190 \\
1 & 3.412960 & 2.575602 & 1.913171 \\
1 & 3.067760 & 4.309517 & 1.987018 \\
6 & 0.702114 & 4.851418 & -1.427669 \\
1 & 0.436382 & 5.533313 & -0.612149 \\
1 & 0.962699 & 5.476529 & -2.292181 \\
1 & -0.203215 & 4.290112 & -1.693499 \\
6 & 3.060474 & 4.810108 & -0.620793 \\
1 & 3.935955 & 4.204480 & -0.352795 \\
1 & 3.362456 & 5.483623 & -1.434176 \\
1 & 2.812193 & 5.445155 & 0.238835 \\
6 & 0.694436 & 1.599099 & -2.230792 \\
1 & 0.471219 & 2.484706 & -2.849466 \\
1 & 1.738901 & 1.329717 & -2.431465 \\
1 & -0.563639 & 4.132893 & 1.108838 \\
1 & 0.410758 & 0.897546 & 0.724007 \\
1 & 0.074721 & 0.781168 & -2.622769 \\
6 & 1.571566 & 2.744933 & -0.131165 \\
1 & 2.497007 & 2.140931 & -0.144894 \\
& & & \\
\hline
\end{tabular}

$5^{\prime}$

The smallest/imaginary frequencies is: $31.3610 \mathrm{~cm}(-1)$

$\begin{array}{lc}\text { Zero-point correction }= & 0.886937 \\ \text { Thermal correction to Energy= } & 0.940740 \\ \text { Thermal correction to Enthalpy= } & 0.941764 \\ \text { Thermal correction to Gibbs Free Energy= } & 0.805191\end{array}$


Sum of electronic and zero-point Energies= Sum of electronic and thermal Energies= Sum of electronic and thermal Enthalpies= Sum of electronic and thermal Free Energies=
$-2080.652046$

$-2080.598242$

$-2080.597219$

$-2080.733792$

Cartesian Coordinates

$\begin{array}{llll}6 & 0.541809 & -2.277069 & -0.374749 \\ 15 & 2.488486 & -0.159363 & -0.027133 \\ 15 & -2.074626 & -1.160353 & 0.209995 \\ 6 & 2.858646 & -1.638962 & -1.084093 \\ 6 & 3.366244 & 1.210797 & -1.023023 \\ 6 & 3.417048 & -0.488629 & 1.595381 \\ 6 & -1.692531 & -2.958773 & 0.487976 \\ 6 & -3.464147 & -0.934606 & 1.514470 \\ 6 & -2.784201 & -1.135099 & -1.578488 \\ 6 & 1.789098 & -2.676809 & -0.903203 \\ 6 & 2.054316 & -4.001580 & -1.259796 \\ 6 & 1.080319 & -4.981409 & -1.105063 \\ 6 & -0.404426 & -3.307953 & -0.189310 \\ 6 & -0.151321 & -4.629475 & -0.563695 \\ 1 & 3.032711 & -4.265653 & -1.664659 \\ 1 & 1.283123 & -6.011457 & -1.392155 \\ 1 & -0.919016 & -5.390195 & -0.412330 \\ 1 & 2.861860 & -1.279269 & -2.123499 \\ 1 & 3.867616 & -2.035127 & -0.898456 \\ 1 & -1.568336 & -3.073731 & 1.574038 \\ 1 & -2.516277 & -3.619936 & 0.194603 \\ 6 & -4.519238 & -2.036691 & 1.398997 \\ 1 & -5.027466 & -2.030597 & 0.426438 \\ 1 & -4.109836 & -3.039153 & 1.564412 \\ 1 & -5.290954 & -1.875872 & 2.165983 \\ 6 & -3.203804 & -2.519215 & -2.069547 \\ 1 & -2.371946 & -3.233190 & -2.077682 \\ 1 & -4.021272 & -2.944327 & -1.473511 \\ 1 & -3.568551 & -2.431940 & -3.103601 \\ 6 & 4.689586 & 0.769737 & -1.656407 \\ 1 & 5.439528 & 0.461662 & -0.922112 \\ 1 & 4.566125 & -0.043821 & -2.379501 \\ 1 & 5.109571 & 1.623542 & -2.208151 \\ 6 & 4.937472 & -0.500191 & 1.448478 \\ 1 & 5.284427 & -1.221786 & 0.698033 \\ 1 & 5.347684 & 0.484910 & 1.200883 \\ 1 & 5.381471 & -0.799699 & 2.408797 \\ 6 & -2.782824 & -1.030882 & 2.884487 \\ 1 & -2.298817 & -1.997571 & 3.063002 \\ 1 & -2.030588 & -0.244833 & 3.019767 \\ 1 & -3.542043 & -0.904643 & 3.669457 \\ 6 & -1.656642 & -0.617410 & -2.481751 \\ 1 & -0.810080 & -1.313030 & -2.523166 \\ 1 & -2.035393 & -0.488956 & -3.507116 \\ 1 & -1.285409 & 0.366236 & -2.150845 \\ 6 & 2.401956 & 1.565751 & -2.158804\end{array}$

\begin{tabular}{|c|c|c|c|}
\hline 1 & 2.160243 & 0.697923 & -2.789082 \\
\hline 1 & 1.459464 & 1.979568 & -1.786298 \\
\hline 1 & 2.864297 & 2.320843 & -2.811094 \\
\hline 6 & 2.984491 & -1.864134 & 2.110501 \\
\hline 1 & 3.295409 & -2.679256 & 1.445743 \\
\hline 1 & 3.458217 & -2.035750 & 3.087718 \\
\hline 1 & 1.899436 & -1.937237 & 2.243839 \\
\hline 6 & -4.179243 & 0.416807 & 1.468005 \\
\hline 1 & -4.860093 & 0.474816 & 2.329334 \\
\hline 1 & -3.488401 & 1.260276 & 1.551012 \\
\hline 1 & -4.787144 & 0.558945 & 0.570743 \\
\hline 6 & -3.980123 & -0.198524 & -1.725144 \\
\hline 1 & -4.874995 & -0.585572 & -1.224200 \\
\hline 1 & -3.776890 & 0.808496 & -1.349057 \\
\hline 1 & -4.225294 & -0.103112 & -2.793252 \\
\hline 6 & 3.637560 & 2.455996 & -0.178372 \\
\hline 1 & 3.942528 & 3.280031 & -0.840320 \\
\hline 1 & 2.759891 & 2.794824 & 0.382394 \\
\hline 1 & 4.457186 & 2.300108 & 0.533207 \\
\hline 6 & 3.017162 & 0.562351 & 2.631411 \\
\hline 1 & 3.326256 & 1.575537 & 2.350572 \\
\hline 1 & 1.933343 & 0.573499 & 2.797305 \\
\hline 1 & 3.501763 & 0.325211 & 3.589565 \\
\hline 77 & 0.128518 & -0.240963 & 0.178382 \\
\hline 6 & -0.082367 & 1.902369 & 0.780710 \\
\hline 1 & 0.960106 & 2.223273 & 0.931048 \\
\hline 6 & 0.195008 & 4.166445 & -0.440151 \\
\hline 1 & 1.222835 & 3.821063 & -0.644304 \\
\hline 6 & -2.167993 & 2.952650 & -0.384311 \\
\hline 1 & -2.504655 & 1.958242 & -0.049156 \\
\hline 6 & 0.299355 & 5.009615 & 0.827792 \\
\hline 1 & 0.706090 & 4.438249 & 1.669884 \\
\hline 1 & 0.975570 & 5.858292 & 0.654639 \\
\hline 6 & -0.170225 & 5.056570 & -1.624730 \\
\hline 1 & 0.593115 & 5.833962 & -1.765364 \\
\hline 1 & -0.233031 & 4.485926 & -2.561406 \\
\hline 1 & -1.125937 & 5.577437 & -1.479890 \\
\hline 6 & -2.831252 & 3.979469 & 0.531312 \\
\hline 1 & -2.657343 & 5.005629 & 0.180296 \\
\hline 1 & -3.920669 & 3.827837 & 0.530842 \\
\hline 1 & -2.492855 & 3.919859 & 1.570122 \\
\hline 6 & -2.737275 & 3.136285 & -1.793353 \\
\hline 1 & -2.543891 & 4.137542 & -2.194376 \\
\hline 1 & -2.324809 & 2.410091 & -2.507869 \\
\hline 1 & -3.829410 & 3.007616 & -1.787663 \\
\hline 6 & -0.726456 & 2.090903 & 2.154168 \\
\hline
\end{tabular}




$\begin{array}{lrrrrrrr}1 & -0.194960 & 1.487432 & 2.904967 & 1 & -0.665524 & 5.427366 & 1.139735 \\ 1 & -0.712276 & 3.128986 & 2.529408 & 6 & -0.623677 & 2.847314 & -0.340404 \\ 1 & -1.771916 & 1.761918 & 2.191818 & 1 & -0.386337 & 2.344704 & -1.299846 \\ 1 & 0.304802 & -0.560133 & 1.676290 & & & & \end{array}$

TS2'

The smallest/imaginary frequencies is: $-742.7978 \mathrm{~cm}(-1)$

$\begin{array}{lc}\text { Zero-point correction }= & 0.883398 \\ \text { Thermal correction to Energy= } & 0.936456 \\ \text { Thermal correction to Enthalpy= } & 0.937479 \\ \text { Thermal correction to Gibbs Free Energy= } & 0.801599 \\ \text { Sum of electronic and zero-point Energies }= & -2080.647257 \\ \text { Sum of electronic and thermal Energies }= & -2080.594199 \\ \text { Sum of electronic and thermal Enthalpies }= & -2080.593176 \\ \text { Sum of electronic and thermal Free Energies= } & -2080.729056\end{array}$

Cartesian Coordinates

\begin{tabular}{|c|c|c|c|c|c|c|c|}
\hline 6 & 1.055333 & -2.020549 & 0.030367 & 1 & 5.608043 & -0.308769 & -1.934153 \\
\hline 15 & 2.471408 & 0.454417 & 0.145721 & 6 & 4.455883 & 1.893162 & 1.642380 \\
\hline 15 & -1.760232 & -1.529633 & -0.133476 & 1 & 4.672393 & 0.981141 & 2.211749 \\
\hline 6 & 2.991974 & -0.980739 & 1.190775 & 1 & 5.164475 & 1.959068 & 0.808547 \\
\hline 6 & 3.612430 & 0.283792 & -1.396571 & 1 & 4.662898 & 2.745933 & 2.305659 \\
\hline 6 & 3.000667 & 1.970359 & 1.180214 & 6 & -1.133710 & -2.746962 & 2.339266 \\
\hline 6 & -1.000459 & -3.029524 & -0.927722 & 1 & -0.754864 & -3.661830 & 1.869000 \\
\hline 6 & -2.326126 & -2.145613 & 1.588567 & 1 & -0.303398 & -2.038380 & 2.428637 \\
\hline 6 & -3.323198 & -1.323523 & -1.237524 & 1 & -1.467793 & -3.008486 & 3.353934 \\
\hline 6 & 2.324699 & -2.196956 & 0.618446 & 6 & -2.901319 & -0.529147 & -2.473836 \\
\hline 6 & 2.932076 & -3.451588 & 0.686740 & 1 & -2.011518 & -0.942152 & -2.966264 \\
\hline 6 & 2.279858 & -4.575943 & 0.188614 & 1 & -3.721586 & -0.542994 & -3.206061 \\
\hline 6 & 0.401309 & -3.179034 & -0.422177 & 1 & -2.705066 & 0.517305 & -2.223980 \\
\hline 6 & 1.006705 & -4.436093 & -0.353354 & 6 & 2.950637 & -0.642136 & -2.421473 \\
\hline 1 & 3.920248 & -3.548041 & 1.139576 & 1 & 2.717770 & -1.630076 & -2.009185 \\
\hline 1 & 2.753991 & -5.554283 & 0.237225 & 1 & 2.022660 & -0.220078 & -2.821961 \\
\hline 1 & 0.470307 & -5.313116 & -0.718795 & 1 & 3.649161 & -0.780625 & -3.259783 \\
\hline 1 & 4.078752 & -1.088131 & 1.281963 & 6 & 2.100102 & 1.974955 & 2.420110 \\
\hline 1 & 2.594845 & -0.778755 & 2.196577 & 1 & 2.249966 & 1.092784 & 3.053664 \\
\hline 1 & -1.598605 & -3.938492 & -0.775683 & 1 & 2.342175 & 2.857806 & 3.029097 \\
\hline 1 & -1.003261 & -2.816902 & -2.008653 & 1 & 1.038129 & 2.013449 & 2.155821 \\
\hline 6 & -3.409241 & -3.223558 & 1.529936 & 6 & -2.834733 & -0.958733 & 2.407109 \\
\hline 1 & -4.363631 & -2.859577 & 1.135965 & 1 & -3.120896 & -1.310292 & 3.409020 \\
\hline 1 & -3.099680 & -4.098715 & 0.944830 & 1 & -2.051576 & -0.201117 & 2.528794 \\
\hline 1 & -3.602916 & -3.577783 & 2.552639 & 1 & -3.715494 & -0.478467 & 1.966650 \\
\hline 6 & -3.890572 & -2.660198 & -1.729205 & 6 & -4.443253 & -0.545508 & -0.547588 \\
\hline 1 & -3.201359 & -3.182583 & -2.401626 & 1 & -5.210605 & -0.293271 & -1.294455 \\
\hline 1 & -4.167359 & -3.346245 & -0.924319 & 1 & -4.940189 & -1.121362 & 0.240622 \\
\hline 1 & -4.803280 & -2.453561 & -2.306953 & 1 & -4.094509 & 0.398398 & -0.113177 \\
\hline 6 & 4.968686 & -0.330781 & -1.039776 & 6 & 3.851836 & 1.631509 & -2.077072 \\
\hline 1 & 5.500520 & 0.208915 & -0.248483 & 1 & 4.316456 & 1.453295 & -3.057401 \\
\hline 1 & 4.874038 & -1.380859 & -0.739008 & 1 & 2.925021 & 2.191680 & -2.254736 \\
\hline
\end{tabular}




$\begin{array}{cccccccc}1 & 4.539228 & 2.269538 & -1.511244 & 1 & -3.564064 & 4.322065 & -2.542486 \\ 6 & 2.805139 & 3.303309 & 0.444335 & 1 & -2.608858 & 2.853823 & -2.771002 \\ 1 & 3.621334 & 3.520756 & -0.249694 & 1 & -4.078415 & 2.806666 & -1.777078 \\ 1 & 1.867656 & 3.367064 & -0.119431 & 6 & -1.238060 & 4.699682 & -1.186627 \\ 1 & 2.793090 & 4.115624 & 1.185200 & 1 & -0.575668 & 4.238776 & -1.932231 \\ 77 & 0.169989 & -0.095784 & -0.107827 & 1 & -1.635952 & 5.621423 & -1.631421 \\ 6 & -0.210747 & 1.509376 & -1.677568 & 1 & -0.618607 & 4.989097 & -0.329277 \\ 1 & 0.145984 & 0.036596 & -1.839517 & 6 & -1.365735 & 3.866138 & 2.085626 \\ 6 & -0.553251 & 1.996283 & -0.361424 & 1 & -1.812601 & 4.813637 & 1.753265 \\ 1 & 0.130942 & -0.156195 & 1.531247 & 1 & -1.418690 & 3.855366 & 3.182256 \\ 1 & 0.205419 & 2.640262 & 0.080043 & 1 & -0.301981 & 3.884776 & 1.814393 \\ 1 & 0.706037 & 1.884292 & -2.142356 & 6 & -3.565526 & 2.705560 & 1.937873 \\ 1 & -1.008319 & 1.414163 & -2.415183 & 1 & -4.076446 & 3.606815 & 1.570157 \\ 6 & -2.103640 & 2.660928 & 1.509214 & 1 & -4.127017 & 1.837037 & 1.567415 \\ 1 & -1.652007 & 1.758574 & 1.949906 & 1 & -3.654379 & 2.712055 & 3.032387 \\ 6 & -2.380896 & 3.765560 & -0.799666 & 6 & -1.946655 & 2.487064 & -0.016863 \\ 1 & -3.050439 & 4.340208 & -0.138265 & 1 & -2.657567 & 1.696259 & -0.302380 \\ 6 & -3.201839 & 3.417260 & -2.036298 & & & & \end{array}$

6

The smallest/imaginary frequencies is: $18.7763 \mathrm{~cm}(-1)$

$\begin{array}{lc}\text { Zero-point correction= } & 0.885772 \\ \text { Thermal correction to Energy= } & 0.938852 \\ \text { Thermal correction to Enthalpy= } & 0.939876 \\ \text { Thermal correction to Gibbs Free Energy= } & 0.804118 \\ \text { Sum of electronic and zero-point Energies }= & -2080.664044 \\ \text { Sum of electronic and thermal Energies }= & -2080.610964 \\ \text { Sum of electronic and thermal Enthalpies }= & -2080.609941 \\ \text { Sum of electronic and thermal Free Energies }= & -2080.745698\end{array}$

Cartesian Coordinates

$\begin{array}{ccccccccc}6 & 1.050649 & -1.901733 & 0.562593 & 1 & 2.421177 & -0.018992 & 2.327154 \\ 15 & 2.460914 & 0.509529 & -0.006918 & 1 & -1.442668 & -4.092633 & -0.033855 \\ 15 & -1.710432 & -1.629648 & -0.218915 & 1 & -0.649462 & -3.396277 & -1.451259 \\ 6 & 2.901900 & -0.511918 & 1.471135 & 6 & -3.820199 & -2.873605 & 1.344804 \\ 6 & 3.659295 & -0.140675 & -1.363601 & 1 & -4.665665 & -2.536879 & 0.734448 \\ 6 & 3.004423 & 2.270932 & 0.507212 & 1 & -3.487892 & -3.852890 & 0.978601 \\ 6 & -0.825558 & -3.249658 & -0.375000 & 1 & -4.205324 & -3.025685 & 2.363527 \\ 6 & -2.694892 & -1.843577 & 1.405165 & 6 & -3.597078 & -3.062260 & -1.882612 \\ 6 & -2.890733 & -1.712017 & -1.721452 & 1 & -2.892655 & -3.899350 & -1.952372 \\ 6 & 2.279459 & -1.857426 & 1.251156 & 1 & -4.311958 & -3.281719 & -1.087622 \\ 6 & 2.912993 & -3.021615 & 1.688126 & 1 & -4.162607 & -3.042693 & -2.825396 \\ 6 & 2.334290 & -4.265556 & 1.458011 & 6 & 5.039411 & -0.491345 & -0.798987 \\ 6 & 0.483121 & -3.168940 & 0.349644 & 1 & 5.522233 & 0.324112 & -0.254421 \\ 6 & 1.116841 & -4.333045 & 0.791412 & 1 & 4.994219 & -1.367177 & -0.140190 \\ 1 & 3.868234 & -2.949316 & 2.209890 & 1 & 5.700055 & -0.756954 & -1.636803 \\ 1 & 2.827754 & -5.173989 & 1.797658 & 6 & 4.503953 & 2.418436 & 0.769774 \\ 1 & 0.647429 & -5.300540 & 0.608070 & 1 & 4.881675 & 1.683135 & 1.490892 \\ 1 & 3.979084 & -0.563014 & 1.671429 & 1 & 5.105052 & 2.356135 & -0.143929\end{array}$




\begin{tabular}{|c|c|c|c|c|c|c|c|}
\hline 1 & 4.683144 & 3.414031 & 1.202332 & 1 & 2.735492 & 4.320702 & -0.106409 \\
\hline 6 & -1.710941 & -2.284141 & 2.493635 & 77 & 0.149329 & -0.137647 & -0.172984 \\
\hline 1 & -1.322252 & -3.295811 & 2.328985 & 6 & -0.534923 & 1.450325 & -1.574729 \\
\hline 1 & -0.860423 & -1.598493 & 2.581556 & 1 & 0.429466 & -0.830606 & -1.658396 \\
\hline 1 & -2.243127 & -2.288443 & 3.455786 & 6 & -0.690236 & 2.001703 & -0.294431 \\
\hline 6 & -2.066884 & -1.502830 & -2.996308 & 1 & -0.022215 & 0.248246 & 1.461679 \\
\hline 1 & -1.327510 & -2.297810 & -3.150900 & 1 & 0.136565 & 2.606783 & 0.069113 \\
\hline 1 & -2.751016 & -1.523352 & -3.856873 & 1 & 0.311335 & 1.725410 & -2.204543 \\
\hline 1 & -1.529411 & -0.552591 & -3.009817 & 1 & -1.425524 & 1.170211 & -2.136290 \\
\hline 6 & 3.118297 & -1.418393 & -2.011684 & 6 & -1.912328 & 2.827072 & 1.773314 \\
\hline 1 & 2.961643 & -2.226176 & -1.288299 & 1 & -1.380746 & 1.982387 & 2.242280 \\
\hline 1 & 2.173290 & -1.246317 & -2.535391 & 6 & -2.598968 & 3.669212 & -0.556750 \\
\hline 1 & 3.860709 & -1.763987 & -2.745776 & 1 & -3.176104 & 4.286122 & 0.150273 \\
\hline 6 & 2.288006 & 2.575181 & 1.830126 & 6 & -3.588087 & 3.196479 & -1.614911 \\
\hline 1 & 2.753945 & 2.047925 & 2.670917 & 1 & -4.039373 & 4.048664 & -2.140300 \\
\hline 1 & 2.370910 & 3.650415 & 2.042905 & 1 & -3.099172 & 2.571615 & -2.375539 \\
\hline 1 & 1.226493 & 2.305684 & 1.819083 & 1 & -4.403479 & 2.610000 & -1.169769 \\
\hline 6 & -3.273755 & -0.498613 & 1.826276 & 6 & -1.567099 & 4.592250 & -1.201550 \\
\hline 1 & -3.786758 & -0.608586 & 2.793141 & 1 & -1.036896 & 4.092657 & -2.022832 \\
\hline 1 & -2.474712 & 0.239208 & 1.949366 & 1 & -2.068449 & 5.471507 & -1.626784 \\
\hline 1 & -4.006928 & -0.108212 & 1.109367 & 1 & -0.814157 & 4.956303 & -0.492810 \\
\hline 6 & -3.937205 & -0.604846 & -1.626292 & 6 & -1.111708 & 4.092537 & 2.074400 \\
\hline 1 & -4.526474 & -0.575187 & -2.554246 & 1 & -1.652818 & 4.996122 & 1.761550 \\
\hline 1 & -4.641703 & -0.767603 & -0.801270 & 1 & -0.938538 & 4.182019 & 3.154732 \\
\hline 1 & -3.479322 & 0.383040 & -1.496015 & 1 & -0.128898 & 4.108337 & 1.587798 \\
\hline 6 & 3.803414 & 0.902907 & -2.471200 & 6 & -3.282262 & 2.943653 & 2.435026 \\
\hline 1 & 4.329008 & 0.448834 & -3.323144 & 1 & -3.837230 & 3.820178 & 2.073050 \\
\hline 1 & 2.828317 & 1.249444 & -2.839812 & 1 & -3.908895 & 2.061807 & 2.254590 \\
\hline 1 & 4.390969 & 1.774213 & -2.161425 & 1 & -3.179272 & 3.061241 & 3.521453 \\
\hline 6 & 2.608150 & 3.318545 & -0.541268 & 6 & -2.014757 & 2.478143 & 0.271466 \\
\hline 1 & 3.242538 & 3.274474 & -1.431122 & 1 & -2.745946 & 1.659305 & 0.191561 \\
\hline 1 & 1.569753 & 3.240519 & -0.877312 & & & & \\
\hline
\end{tabular}

alkene

The smallest/imaginary frequencies is: $80.1719 \mathrm{~cm}(-1)$

Zero-point correction=

Thermal correction to Energy=

Thermal correction to Enthalpy=

Thermal correction to Gibbs Free Energy=

Sum of electronic and zero-point Energies=

Sum of electronic and thermal Energies=

Sum of electronic and thermal Enthalpies=

Sum of electronic and thermal Free Energies=
0.249297

0.262499

0.263522

0.209488

$-353.249356$

$-353.236154$

$-353.235131$

$-353.289165$

Cartesian Coordinates

$\begin{array}{llll}6 & -0.588999 & 2.670428 & 0.091761 \\ 6 & 0.239203 & 1.776331 & -0.444758 \\ 6 & -1.357290 & -0.181651 & -0.184009 \\ 6 & -1.656201 & -0.350043 & 1.302874\end{array}$

$\begin{array}{rrrr}6 & -1.791468 & -1.418721 & -0.965925 \\ 1 & -2.001717 & 0.632485 & -0.550175 \\ 1 & -1.349113 & 0.522869 & 1.891657 \\ 1 & -2.732510 & -0.495063 & 1.461155\end{array}$




$$
\begin{array}{rrr}
-1.149942 & -1.226992 & 1.727344 \\
-1.605512 & -1.297713 & -2.040813 \\
-1.280703 & -2.332723 & -0.643029 \\
-2.868135 & -1.589943 & -0.836511 \\
1.205267 & -0.393033 & 0.354954 \\
2.587100 & 0.153390 & 0.002666 \\
1.235628 & -1.909575 & 0.200270 \\
1.008636 & -0.150550 & 1.412215 \\
2.710575 & 1.207879 & 0.270049 \\
3.366940 & -0.408269 & 0.531252
\end{array}
$$

$\begin{array}{rrrr}1 & 2.785597 & 0.051860 & -1.074919 \\ 1 & 0.341501 & -2.402655 & 0.594025 \\ 1 & 1.336305 & -2.191397 & -0.858252 \\ 1 & 2.096798 & -2.331092 & 0.733932 \\ 1 & -0.358103 & 3.733246 & 0.061080 \\ 1 & -1.526400 & 2.400636 & 0.575825 \\ 1 & 1.148506 & 2.158836 & -0.912847 \\ 6 & 0.088914 & 0.275369 & -0.491759 \\ 1 & 0.304360 & -0.016377 & -1.538436\end{array}$

\section{Geometries from $(\mathrm{Me}){ }_{2} \mathrm{C}(\mathrm{H})\left(\mathrm{CH}_{2} \mathrm{CH}_{3}\right)$ dehydrogenation}

1

The smallest/imaginary frequencies is: $104.9916 \mathrm{~cm}(-1)$
Zero-point correction=
Thermal correction to Energy=
Thermal correction to Enthalpy=

$$
\begin{gathered}
0.159288 \\
0.167269 \\
0.168292
\end{gathered}
$$
Thermal correction to Gibbs Free Energy=
Sum of electronic and zero-point Energies=
Sum of electronic and thermal Energies=
Sum of electronic and thermal Enthalpies=
Sum of electronic and thermal Free Energies=
0.126358
$-197.450330$
$-197.442350$
$-197.441326$
$-197.483260$

Cartesian Coordinates

$\begin{array}{ccccc}6 & -0.742969 & -0.693515 & 0.257224 \\ 1 & -0.752180 & -1.742053 & -0.076993 \\ 6 & 1.744773 & -0.777975 & 0.004054 \\ 1 & 1.686813 & -1.821343 & -0.331196 \\ 6 & 0.605371 & 1.441880 & 0.120829 \\ 1 & -0.239404 & 2.060232 & -0.204578 \\ 6 & -2.069181 & -0.040177 & -0.093135 \\ 1 & -2.165568 & 0.957711 & 0.351344 \\ 1 & -2.180058 & 0.071608 & -1.180307\end{array}$

$\begin{array}{lrrrr}1 & -0.621722 & -0.727876 & 1.352993 \\ 6 & 0.481565 & -0.003285 & -0.343300 \\ 1 & 0.361960 & -0.004253 & -1.440778 \\ 1 & 1.902655 & -0.788705 & 1.092367 \\ 1 & 2.635188 & -0.327908 & -0.452450 \\ 1 & 1.520301 & 1.906718 & -0.267545 \\ 1 & 0.651436 & 1.490449 & 1.219024\end{array}$

$\begin{array}{llll}1 & -2.916779 & -0.636152 & 0.264093\end{array}$

TS1

The smallest/imaginary frequencies is: $-626.5148 \mathrm{~cm}(-1)$
Zero-point correction=
0.772431
Thermal correction to Energy=
0.820453
Thermal correction to Enthalpy=
0.821477
Thermal correction to Gibbs Free Energy= $\quad 0.693815$
Sum of electronic and zero-point Energies $=\quad-1923.647993$
Sum of electronic and thermal Energies $=\quad-1923.599971$ 
Sum of electronic and thermal Enthalpies=

Sum of electronic and thermal Free Energies=
$-1923.598947$

$-1923.726609$

Cartesian Coordinates

6

$-0.000235-2.032651-0.664286$

$\begin{array}{llll}15 & 2.319362 & -0.381933 & 0.003940\end{array}$

$\begin{array}{lllll}15 & -2.319498 & -0.381407 & 0.003840\end{array}$

$\begin{array}{lllll}6 & 2.524003 & -2.060269 & -0.758738\end{array}$

$\begin{array}{lllll}6 & 3.457554 & 0.677108 & -1.090132\end{array}$

$\begin{array}{lllll}6 & 3.049347 & -0.594615 & 1.745378\end{array}$

$\begin{array}{lllll}6 & -2.524426 & -2.059395 & -0.759498\end{array}$

$\begin{array}{lllll}6 & -3.049377 & -0.594664 & 1.745247\end{array}$

$\begin{array}{lllll}6 & -3.457559 & 0.678234 & -1.089790\end{array}$

$\begin{array}{lllll}6 & 1.200978 & -2.771788 & -0.810222\end{array}$

$6 \quad 1.197080-4.151171-1.027832$

$\begin{array}{lllll}6 & -0.000639 & -4.847561 & -1.130667\end{array}$

$\begin{array}{lllll}6 & -1.201651 & -2.771396 & -0.810554\end{array}$

$\begin{array}{lllll}6 & -1.198159 & -4.150779 & -1.028137\end{array}$

$1 \quad 2.145422-4.682956-1.118162$

$1 \quad-0.000794-5.923981-1.292020$

$1 \quad-2.146652-4.682251-1.118716$

$1 \quad 2.892970-1.910546-1.782967$

$\begin{array}{lllll}1 & 3.297872 & -2.651413 & -0.248866\end{array}$

$\begin{array}{lllll}1 & -3.298829 & -2.650417 & -0.250295\end{array}$

$1 \quad-2.892742-1.909096 \quad-1.783882$

$\begin{array}{lllll}6 & -4.553382 & -0.853366 & 1.774178\end{array}$

$\begin{array}{lllll}1 & -5.133119 & 0.041156 & 1.517994\end{array}$

$1 \quad-4.859935-1.668115 \quad 1.105912$

$1 \quad-4.846981 \quad-1.143114 \quad 2.793793$

$6 \begin{array}{llll}1 & -4.834191 & 0.081830 & -1.386189\end{array}$

$1 \quad-4.780303 \quad-0.928354-1.809100$

$\begin{array}{lllll}1 & -5.483103 & 0.047181 & -0.506744\end{array}$

$\begin{array}{lllll}1 & -5.337328 & 0.714311 & -2.132607\end{array}$

$\begin{array}{lllll}6 & 4.833982 & 0.080260 & -1.386601\end{array}$

$\begin{array}{lllll}1 & 5.482926 & 0.045364 & -0.507193\end{array}$

$1 \quad 4.779752 \quad-0.929887 \quad-1.809551$

$\begin{array}{lllll}1 & 5.337293 & 0.712603 & -2.133017\end{array}$

$\begin{array}{llll}6 & 4.553319 & -0.853507 & 1.774227\end{array}$

$14.859680-1.668578 \quad 1.106266$

$\begin{array}{lllll}1 & 5.133115 & 0.040846 & 1.517561\end{array}$

$1 \quad 4.847024-1.142855 \quad 2.793926$

$\begin{array}{lllll}6 & -2.330280 & -1.793423 & 2.370690\end{array}$

$\begin{array}{lllll}1 & -2.577665 & -2.741217 & 1.877178\end{array}$

$\begin{array}{llll}1 & -1.240263 & -1.671273 & 2.338715\end{array}$

$\begin{array}{llll}1 & -2.635216 & -1.881981 & 3.423502\end{array}$

$\begin{array}{lllll}6 & -2.709971 & 0.818219 & -2.421397\end{array}$

$\begin{array}{lllll}1 & -2.628201 & -0.139243 & -2.952238\end{array}$

$\begin{array}{lllll}1 & -3.258867 & 1.509327 & -3.077459\end{array}$ $\begin{array}{lllll}6 & 2.709802 & 0.816968 & -2.421648\end{array}$

$1 \quad 2.626754 \quad-0.140793 \quad-2.951755$

$\begin{array}{lllll}1 & 1.691080 & 1.200249 & -2.289374\end{array}$

$\begin{array}{lllll}1 & 3.259323 & 1.506965 & -3.078355\end{array}$

$\begin{array}{lllll}6 & 2.330125 & -1.793022 & 2.371352\end{array}$

$\begin{array}{lllll}1 & 2.577343 & -2.741058 & 1.878224\end{array}$

$\begin{array}{lllll}1 & 2.635096 & -1.881193 & 3.424187\end{array}$

$\begin{array}{lllll}1 & 1.240123 & -1.670728 & 2.339379\end{array}$

$\begin{array}{lllll}6 & -2.738733 & 0.635231 & 2.598666\end{array}$

$\begin{array}{llll}1 & -3.147706 & 0.485329 & 3.608525\end{array}$

$\begin{array}{lllll}1 & -1.657348 & 0.790720 & 2.697063\end{array}$

$\begin{array}{lllll}1 & -3.182608 & 1.555025 & 2.199728\end{array}$

$\begin{array}{lllll}6 & -3.641585 & 2.058840 & -0.463646\end{array}$

$1 \quad-4.100907 \quad 2.739561 \quad-1.196058$

$\begin{array}{lllll}1 & -4.307754 & 2.026741 & 0.407750\end{array}$

$1 \quad-2.689943 \quad 2.502118 \quad-0.148894$

$\begin{array}{lllll}6 & 3.642072 & 2.057804 & -0.464336\end{array}$

$1 \quad 4.101349 \quad 2.738264 \quad-1.197020$

$\begin{array}{lllll}1 & 2.690631 & 2.501383 & -0.149396\end{array}$

$\begin{array}{lllll}1 & 4.308485 & 2.025693 & 0.406881\end{array}$

$\begin{array}{lllll}6 & 2.738976 & 0.635627 & 2.598398\end{array}$

$\begin{array}{lllll}1 & 3.182888 & 1.555233 & 2.199072\end{array}$

$\begin{array}{lllll}1 & 1.657626 & 0.791265 & 2.696919\end{array}$

$\begin{array}{lllll}1 & 3.148089 & 0.486032 & 3.608245\end{array}$

$77-0.000016 \quad-0.026922-0.031531$

$\begin{array}{lllll}1 & 0.000016 & 0.868649 & 1.287607\end{array}$

$\begin{array}{lllll}6 & 0.000357 & 2.225993 & 0.568117\end{array}$

$\begin{array}{lllll}1 & 0.874187 & 2.531492 & 1.163100\end{array}$

$\begin{array}{lllll}1 & -0.873053 & 2.531846 & 1.163549\end{array}$

$\begin{array}{lllll}6 & 0.000163 & 3.032219 & -0.733877\end{array}$

$\begin{array}{lllll}1 & -0.877419 & 2.771011 & -1.341522\end{array}$

$\begin{array}{lllll}1 & 0.877318 & 2.770694 & -1.342006\end{array}$

$\begin{array}{lllll}6 & 0.000492 & 4.566309 & -0.557126\end{array}$

$\begin{array}{lllll}1 & 0.000188 & 4.980566 & -1.579780\end{array}$

$\begin{array}{lllll}6 & 1.252633 & 5.085182 & 0.137834\end{array}$

$\begin{array}{lllll}1 & 1.260567 & 6.182561 & 0.162154\end{array}$

$\begin{array}{lllll}1 & 1.312185 & 4.739733 & 1.179075\end{array}$

$\begin{array}{lllll}1 & 2.166468 & 4.759510 & -0.376539\end{array}$

$\begin{array}{lllll}6 & -1.250940 & 5.085619 & 0.138788\end{array}$

$\begin{array}{lllll}1 & -1.258637 & 6.183005 & 0.162828\end{array}$

$\begin{array}{lllll}1 & -2.165295 & 4.759992 & -0.374690\end{array}$

$1 \quad-1.309646 \quad 4.740454 \quad 1.180170$ 
The smallest/imaginary frequencies is: $26.7181 \mathrm{~cm}(-1)$

$\begin{array}{lc}\text { Zero-point correction= } & 0.772431 \\ \text { Thermal correction to Energy= } & 0.820865 \\ \text { Thermal correction to Enthalpy= } & 0.821889 \\ \text { Thermal correction to Gibbs Free Energy= } & 0.693964 \\ \text { Sum of electronic and zero-point Energies= } & -1923.661210 \\ \text { Sum of electronic and thermal Energies= } & -1923.612776 \\ \text { Sum of electronic and thermal Enthalpies= } & -1923.611753 \\ \text { Sum of electronic and thermal Free Energies= } & -1923.739677\end{array}$

Cartesian Coordinates

\begin{tabular}{|c|c|c|c|c|c|c|c|}
\hline 6 & -0.034985 & -2.069566 & -0.665646 & 1 & -1.354575 & -1.814067 & 2.370385 \\
\hline 15 & 2.309516 & -0.430404 & -0.029728 & 1 & -2.797907 & -1.965429 & 3.389920 \\
\hline 15 & -2.324556 & -0.371862 & 20.022133 & 6 & -2.575115 & 0.936136 & -2.349974 \\
\hline 6 & 2.476115 & -2.086304 & -0.856939 & 1 & -2.432366 & -0.008237 & -2.891900 \\
\hline 6 & 3.372777 & 0.695137 & -1.122088 & 1 & -3.100448 & 1.625353 & -3.026759 \\
\hline 6 & 3.093986 & -0.698769 & 1.674864 & 1 & -1.582579 & 1.361224 & -2.150141 \\
\hline 6 & -2.550702 & -2.036257 & -0.775031 & 6 & 2.505311 & 0.955254 & -2.359573 \\
\hline 6 & -3.077074 & -0.602204 & 1.748038 & 1 & 2.269864 & 0.033847 & -2.909202 \\
\hline 6 & -3.394768 & 0.754083 & -1.066494 & 1 & 1.557325 & 1.443981 & -2.097199 \\
\hline 6 & 1.156687 & -2.813463 & -0.847283 & 1 & 3.041372 & 1.620908 & -3.051486 \\
\hline 6 & 1.137026 & -4.192154 & -1.067855 & 6 & 2.453531 & -1.953887 & 2.275643 \\
\hline 6 & -0.071448 & -4.876425 & -1.144336 & 1 & 2.705509 & -2.866351 & 1.722248 \\
\hline 6 & -1.246275 & -2.788561 & -0.807189 & 1 & 2.824294 & -2.080697 & 3.302766 \\
\hline 6 & -1.262100 & -4.167559 & -1.027478 & 1 & 1.360149 & -1.881094 & 2.321638 \\
\hline 1 & 2.076831 & -4.734387 & -1.184816 & 6 & -2.716375 & 0.583347 & 2.642443 \\
\hline 1 & -0.085159 & -5.952770 & -1.305617 & 1 & -3.143110 & 0.420680 & 3.642879 \\
\hline 1 & -2.216229 & -4.690373 & -1.112568 & 1 & -1.630791 & 0.691877 & 2.752065 \\
\hline 1 & 2.767134 & -1.889175 & -1.898778 & 1 & -3.112970 & 1.534063 & 2.267442 \\
\hline 1 & 3.296638 & -2.679971 & -0.429188 & 6 & -3.587339 & 2.112352 & -0.393286 \\
\hline 1 & -3.363347 & -2.608438 & -0.305272 & 1 & -4.013755 & 2.822735 & -1.117029 \\
\hline 1 & -2.880285 & -1.850305 & -1.807299 & 1 & -4.286444 & 2.052666 & 0.450481 \\
\hline 6 & -4.593915 & -0.774839 & 1.730950 & 1 & -2.644680 & 2.536886 & -0.028098 \\
\hline 1 & -5.112170 & 0.152746 & 1.460942 & 6 & 3.638010 & 2.021180 & -0.410820 \\
\hline 1 & -4.924195 & -1.569155 & 1.049314 & 1 & 4.048430 & 2.746424 & -1.128932 \\
\hline 1 & -4.933715 & -1.050979 & 2.739694 & 1 & 2.725157 & 2.454746 & 0.014564 \\
\hline 6 & -4.762598 & 0.191388 & -1.453614 & 1 & 4.375083 & 1.912386 & 0.394776 \\
\hline 1 & -4.697862 & -0.786685 & -1.944907 & 6 & 2.777894 & 0.482393 & 2.592392 \\
\hline 1 & -5.441364 & 0.098575 & -0.601055 & 1 & 3.196962 & 1.427550 & 2.228087 \\
\hline 1 & -5.236363 & 0.878865 & -2.169785 & 1 & 1.697043 & 0.620067 & 2.716905 \\
\hline 6 & 4.701864 & 0.097590 & -1.583327 & 1 & 3.211783 & 0.291208 & 3.584659 \\
\hline 1 & 5.396728 & -0.082879 & -0.757768 & 77 & 0.000191 & -0.033769 & -0.0339 \\
\hline 1 & 4.577350 & -0.842114 & -2.134288 & 6 & -0.012986 & 3.151032 & -0.39543 \\
\hline 1 & 5.189510 & 0.807691 & -2.267360 & 1 & -0.831829 & 2.953210 & -1.1086 \\
\hline 6 & 4.605866 & -0.904673 & 1.619688 & 6 & -0.544192 & 5.530497 & -1.01609 \\
\hline 1 & 4.899837 & -1.705300 & 0.928839 & 1 & -1.461963 & 5.222009 & $-1.5354 \varepsilon$ \\
\hline 1 & 5.139199 & 0.010557 & 1.337223 & 6 & 1.016595 & 5.080545 & 0.882459 \\
\hline 1 & 4.964767 & -1.190945 & 2.618937 & 1 & 1.278808 & 4.421803 & 1.71950 \\
\hline 6 & -2.450192 & -1.862623 & 2.352094 & 6 & 0.039412 & 2.056136 & 0.673107 \\
\hline 1 & -2.736327 & -2.777362 & 1.819494 & 1 & 0.012242 & -0.402462 & 1.460419 \\
\hline
\end{tabular}




$\begin{array}{rrrrrrrr}1 & 0.931081 & 2.229876 & 1.297315 & 1 & 0.267268 & 5.545200 & -1.758979 \\ 1 & -0.809236 & 2.236015 & 1.356678 & 1 & -0.687493 & 6.561397 & -0.666348 \\ 1 & 0.906751 & 3.148676 & -1.010645 & 1 & 0.858639 & 6.088252 & 1.288486 \\ 6 & -0.206994 & 4.582789 & 0.126336 & 1 & 1.886547 & 5.130749 & 0.209494 \\ 1 & -1.062372 & 4.565760 & 0.825602 & & & & \end{array}$

TS2

The smallest/imaginary frequencies is: $-733.7330 \mathrm{~cm}(-1)$

Zero-point correction=

Thermal correction to Energy=

Thermal correction to Enthalpy=

Thermal correction to Gibbs Free Energy=

Sum of electronic and zero-point Energies=

Sum of electronic and thermal Energies=

Sum of electronic and thermal Enthalpies=

Sum of electronic and thermal Free Energies=
0.770295

0.817220

0.818243

0.694942

$-1923.632672$

$-1923.585747$

$-1923.584724$

$-1923.708025$

Cartesian Coordinates

$\begin{array}{cccc}6 & 0.016104 & -1.796782 & -0.593716 \\ 15 & 2.321348 & -0.278276 & 0.250664 \\ 15 & -2.347596 & -0.257748 & 0.044730 \\ 6 & 2.290425 & -2.119666 & 0.382540 \\ 6 & 3.292441 & 0.003736 & -1.388227 \\ 6 & 3.391250 & 0.260050 & 1.734300 \\ 6 & -2.394023 & -1.601562 & -1.233440 \\ 6 & -3.116141 & -1.033689 & 1.609131 \\ 6 & -3.568725 & 0.991562 & -0.731472 \\ 6 & 1.149097 & -2.629756 & -0.449650 \\ 6 & 1.189092 & -3.919049 & -0.980972 \\ 6 & 0.076749 & -4.448315 & -1.628199 \\ 6 & -1.115550 & -2.385448 & -1.191004 \\ 6 & -1.081908 & -3.684761 & -1.704582 \\ 1 & 2.092497 & -4.520101 & -0.864334 \\ 1 & 0.105000 & -5.453879 & -2.043327 \\ 1 & -1.978612 & -4.100633 & -2.166954 \\ 1 & 3.248177 & -2.595751 & 0.144178 \\ 1 & 2.078178 & -2.335405 & 1.439941 \\ 1 & -3.278898 & -2.244521 & -1.120949 \\ 1 & -2.497669 & -1.091449 & -2.202056 \\ 6 & -4.622153 & -1.275766 & 1.529718 \\ 1 & -5.198510 & -0.344678 & 1.559847 \\ 1 & -4.918645 & -1.845095 & 0.639941 \\ 1 & -4.927361 & -1.865527 & 2.406145 \\ 6 & -4.924917 & 0.413824 & -1.151041 \\ 1 & -4.829011 & -0.499597 & -1.750279 \\ 1 & -5.590959 & 0.206275 & -0.311220 \\ 1 & -5.430072 & 1.158403 & -1.784024 \\ 6 & 4.350459 & -1.078565 & -1.623441\end{array}$

$\begin{array}{lrrr}1 & 5.053467 & -1.196540 & -0.792537 \\ 1 & 3.895383 & -2.053948 & -1.833293 \\ 1 & 4.938802 & -0.803410 & -2.510636 \\ 6 & 4.783842 & -0.368809 & 1.732384 \\ 1 & 4.751036 & -1.464385 & 1.679938 \\ 1 & 5.409829 & -0.000598 & 0.911161 \\ 1 & 5.296525 & -0.105322 & 2.669216 \\ 6 & -2.445036 & -2.390133 & 1.845569 \\ 1 & -2.693845 & -3.120368 & 1.065583 \\ 1 & -1.354447 & -2.311088 & 1.906054 \\ 1 & -2.807679 & -2.794305 & 2.801708 \\ 6 & -2.909608 & 1.503318 & -2.020945 \\ 1 & -3.083666 & 0.817060 & -2.858471 \\ 1 & -3.352121 & 2.469738 & -2.303024 \\ 1 & -1.828500 & 1.630685 & -1.927828 \\ 6 & 2.314260 & -0.062848 & -2.564236 \\ 1 & 1.738253 & -0.994716 & -2.578539 \\ 1 & 1.600124 & 0.766902 & -2.554790 \\ 1 & 2.893323 & -0.006587 & -3.497875 \\ 6 & 2.657966 & -0.188040 & 3.002768 \\ 1 & 2.609937 & -1.278564 & 3.102808 \\ 1 & 3.205278 & 0.191727 & 3.877116 \\ 1 & 1.632689 & 0.197545 & 3.043908 \\ 6 & -2.826690 & -0.129787 & 2.808268 \\ 1 & -3.233848 & -0.596389 & 3.717157 \\ 1 & -1.750120 & 0.016942 & 2.950941 \\ 1 & -3.294609 & 0.857869 & 2.710642 \\ 6 & -3.812974 & 2.148803 & 0.235753 \\ 1 & -4.420674 & 2.920643 & -0.258752 \\ 1 & -4.365851 & 1.824777 & 1.126238\end{array}$




$\begin{array}{cccc}1 & -2.884187 & 2.624074 & 0.573097 \\ 6 & 3.974537 & 1.371039 & -1.427727 \\ 1 & 4.348861 & 1.547917 & -2.446413 \\ 1 & 3.287831 & 2.193980 & -1.190275 \\ 1 & 4.838004 & 1.432038 & -0.755403 \\ 6 & 3.535736 & 1.782958 & 1.805476 \\ 1 & 4.148942 & 2.195056 & 1.000054 \\ 1 & 2.568473 & 2.296916 & 1.793893 \\ 1 & 4.030526 & 2.044784 & 2.751808 \\ 77 & -0.012368 & 0.153290 & 0.252616 \\ 6 & 0.348027 & 2.435999 & -0.062483 \\ 1 & 0.118668 & 1.270479 & -1.099261 \\ 6 & 0.083420 & 3.560955 & -2.318717 \\ 1 & -0.119353 & 2.619515 & -2.844380\end{array}$

$\begin{array}{rrrr}6 & -0.052548 & 4.859560 & -0.192794 \\ 1 & -0.415718 & 4.885601 & 0.841059 \\ 6 & -0.071557 & 2.105833 & 1.270811 \\ 1 & -0.056704 & -0.722627 & 1.628649 \\ 1 & 0.653747 & 2.151201 & 2.081650 \\ 1 & -1.064366 & 2.445108 & 1.575035 \\ 1 & 1.433746 & 2.533553 & -0.190342 \\ 6 & -0.355878 & 3.521452 & -0.863736 \\ 1 & -1.441297 & 3.356784 & -0.814259 \\ 1 & -0.518713 & 5.687780 & -0.740977 \\ 1 & 1.031240 & 5.045499 & -0.170799 \\ 1 & 1.164965 & 3.751852 & -2.387366 \\ 1 & -0.426174 & 4.365100 & -2.863739\end{array}$

TS1'

The smallest/imaginary frequencies is: $-556.8471 \mathrm{~cm}(-1)$
Zero-point correction=
Thermal correction to Energy=
Thermal correction to Enthalpy=
0.772894
0.820588
0.821612
Thermal correction to Gibbs Free Energy $=\quad 0.695830$
Sum of electronic and zero-point Energies $=\quad-1923.642135$
Sum of electronic and thermal Energies $=\quad-1923.594440$
Sum of electronic and thermal Enthalpies $=\quad-1923.593417$
Sum of electronic and thermal Free Energies $=\quad-1923.719199$

Cartesian Coordinates

\begin{tabular}{ccccccccc}
\hline 6 & 0.075090 & 2.034959 & -0.070839 & 1 & -5.545385 & 0.279424 & -0.382882 \\
15 & 2.351773 & 0.194038 & 0.083022 & 1 & -4.806213 & 1.551408 & -1.371439 \\
15 & -2.310398 & 0.352639 & -0.005880 & 1 & -5.516366 & 0.131643 & -2.138322 \\
6 & 2.529395 & 1.992117 & 0.495909 & 6 & -4.450780 & 0.567033 & 1.945242 \\
6 & 3.280284 & -0.621630 & 1.535512 & 1 & -4.710419 & 1.539037 & 1.507586 \\
6 & 3.365329 & 0.042139 & -1.523991 & 1 & -5.116957 & -0.194030 & 1.522888 \\
6 & -2.426856 & 2.172276 & -0.364704 & 1 & -4.677239 & 0.626430 & 3.019906 \\
6 & -3.605739 & -0.296953 & -1.256285 & 6 & 4.573382 & 0.092845 & 1.937545 \\
6 & -2.977463 & 0.207177 & 1.773331 & 1 & 5.333510 & 0.081424 & 1.151550 \\
6 & 1.284531 & 2.745670 & 0.130148 & 1 & 4.410902 & 1.134080 & 2.237744 \\
6 & 1.340212 & 4.139928 & 0.092775 & 1 & 5.001606 & -0.425972 & 2.807645 \\
6 & 0.183949 & 4.886400 & -0.100704 & 6 & 4.848615 & 0.373916 & -1.376694 \\
6 & -1.085695 & 2.829754 & -0.221537 & 1 & 5.031450 & 1.334185 & -0.878772 \\
6 & -1.029833 & 4.226193 & -0.236532 & 1 & 5.394807 & -0.407421 & -0.835271 \\
1 & 2.296297 & 4.644016 & 0.241137 & 1 & 5.297876 & 0.440261 & -2.378556 \\
1 & 0.226976 & 5.973742 & -0.126197 & 6 & -2.974002 & -0.127075 & -2.643124 \\
1 & -1.950555 & 4.798330 & -0.360601 & 1 & -2.886820 & 0.927659 & -2.931997 \\
1 & 2.659073 & 2.050906 & 1.585723 & 1 & -1.976403 & -0.568938 & -2.711771 \\
1 & 3.437605 & 2.433326 & 0.061542 & 1 & -3.614650 & -0.613123 & -3.393157 \\
1 & -2.784934 & 2.281651 & -1.396857 & 6 & -2.140081 & 1.158386 & 2.633606 \\
1 & -3.186707 & 2.657980 & 0.265238 & 1 & -2.304191 & 2.213107 & 2.380073 \\
6 & -4.932987 & 0.467714 & -1.268315 & 1 & -2.424747 & 1.025038 & 3.687616
\end{tabular}




$\begin{array}{lrrr}1 & -1.066680 & 0.950251 & 2.536364 \\ 6 & 2.324623 & -0.573385 & 2.731552 \\ 1 & 2.013976 & 0.449879 & 2.981074 \\ 1 & 1.413328 & -1.154244 & 2.549974 \\ 1 & 2.830838 & -0.990692 & 3.614304 \\ 6 & 2.733769 & 1.034969 & -2.504999 \\ 1 & 2.860418 & 2.078272 & -2.190748 \\ 1 & 3.217862 & 0.924965 & -3.486212 \\ 1 & 1.658688 & 0.854794 & -2.635191 \\ 6 & -3.910500 & -1.773084 & -1.016941 \\ 1 & -4.561156 & -2.146668 & -1.821138 \\ 1 & -3.007913 & -2.394562 & -1.009084 \\ 1 & -4.443860 & -1.935016 & -0.071708 \\ 6 & -2.752047 & -1.211820 & 2.294095 \\ 1 & -3.121548 & -1.279967 & 3.327837 \\ 1 & -3.280110 & -1.973160 & 1.706939 \\ 1 & -1.685467 & -1.464248 & 2.300991 \\ 6 & 3.611433 & -2.081149 & 1.231688 \\ 1 & 3.967565 & -2.570525 & 2.150016 \\ 1 & 2.739201 & -2.643852 & 0.878458 \\ 1 & 4.410072 & -2.177067 & 0.485876 \\ 6 & 3.241854 & -1.357510 & -2.124541\end{array}$

$\begin{array}{cccc}1 & 3.668526 & -2.136317 & -1.482776 \\ 1 & 2.201572 & -1.621112 & -2.337462 \\ 1 & 3.790580 & -1.384931 & -3.077432 \\ 77 & 0.022493 & -0.076864 & -0.099505 \\ 6 & 0.140461 & -2.344338 & -0.573169 \\ 1 & 1.213057 & -2.576859 & -0.487414 \\ 6 & -0.001179 & -3.724619 & 1.566683 \\ 1 & 0.035640 & -2.822711 & 2.190924 \\ 6 & -0.584944 & -4.793473 & -0.580414 \\ 1 & -1.142673 & -4.746059 & -1.522357 \\ 6 & -0.215866 & -2.349000 & -2.057080 \\ 1 & 0.244056 & -3.206319 & -2.576798 \\ 1 & -1.298184 & -2.429052 & -2.214869 \\ 1 & -0.036254 & -1.395933 & 0.784562 \\ 1 & 0.119761 & -1.442677 & -2.577711 \\ 6 & -0.593963 & -3.470413 & 0.185816 \\ 1 & -1.646585 & -3.168047 & 0.321276 \\ 1 & 1.027142 & -4.106830 & 1.479664 \\ 1 & -0.583359 & -4.476978 & 2.114278 \\ 1 & -1.031470 & -5.594333 & 0.023143 \\ 1 & 0.445894 & -5.097969 & -0.817060\end{array}$

$5^{\prime}$

The smallest/imaginary frequencies is: $33.9130 \mathrm{~cm}(-1)$

$\begin{array}{lc}\text { Zero-point correction }= & 0.773885 \\ \text { Thermal correction to Energy= } & 0.821852 \\ \text { Thermal correction to Enthalpy= } & 0.822875 \\ \text { Thermal correction to Gibbs Free Energy= } & 0.698027 \\ \text { Sum of electronic and zero-point Energies= } & -1923.642762 \\ \text { Sum of electronic and thermal Energies= } & -1923.594796 \\ \text { Sum of electronic and thermal Enthalpies }= & -1923.593772 \\ \text { Sum of electronic and thermal Free Energies }= & -1923.718620\end{array}$

Cartesian Coordinates

$\begin{array}{ccccccccc}6 & -0.039279 & 1.956402 & -0.187404 & 1 & -2.108443 & 4.542929 & -1.106931 \\ 15 & -2.362708 & 0.228630 & -0.028745 & 1 & -0.044178 & 5.862874 & -0.684660 \\ 15 & 2.307728 & 0.269379 & 0.151234 & 1 & 2.009900 & 4.701236 & 0.105237 \\ 6 & -2.442410 & 1.872573 & -0.887760 & 1 & -2.510272 & 1.650325 & -1.962950 \\ 6 & -3.486343 & -0.818432 & -1.162226 & 1 & -3.356909 & 2.428113 & -0.634051 \\ 6 & -3.206929 & 0.520837 & 1.644234 & 1 & 2.227831 & 2.061199 & 1.746365 \\ 6 & 2.303035 & 2.063357 & 0.649715 & 1 & 3.237783 & 2.579093 & 0.400589 \\ 6 & 3.616768 & -0.384675 & 1.387186 & 6 & 4.878882 & 0.479672 & 1.374127 \\ 6 & 2.992453 & 0.313359 & -1.651028 & 1 & 5.374558 & 0.481629 & 0.395112 \\ 6 & -1.191543 & 2.655301 & -0.609536 & 1 & 4.687272 & 1.518950 & 1.662352 \\ 6 & -1.195816 & 4.041971 & -0.779847 & 1 & 5.598597 & 0.072872 & 2.099396 \\ 6 & -0.044105 & 4.783853 & -0.542000 & 6 & 3.662435 & 1.645879 & -1.983548 \\ 6 & 1.097901 & 2.746853 & 0.082876 & 1 & 2.980767 & 2.497498 & -1.876657 \\ 6 & 1.104184 & 4.130569 & -0.106822 & 1 & 4.552082 & 1.831587 & -1.368330\end{array}$




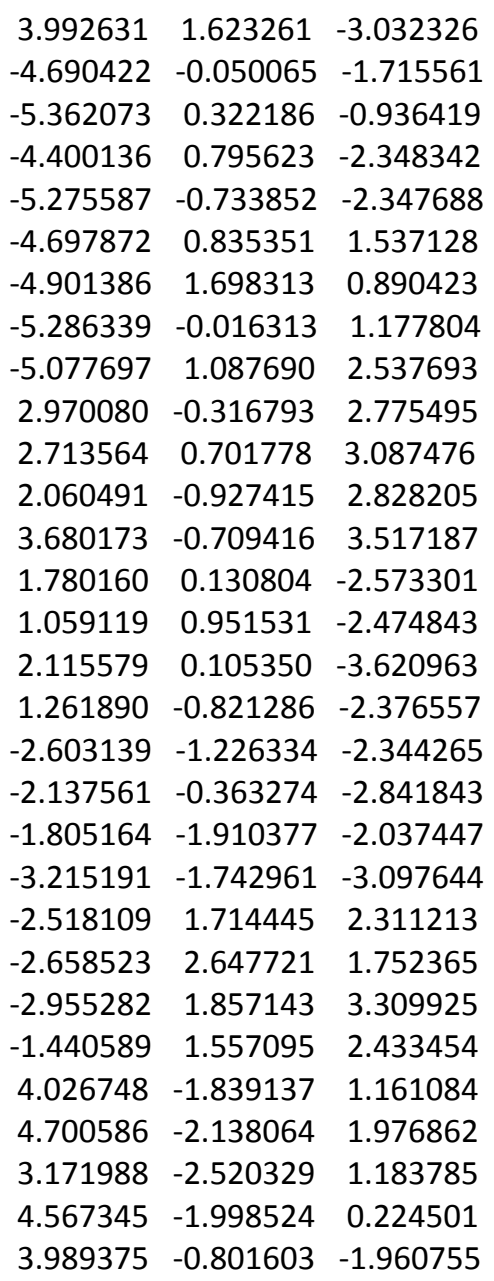

TS2'

The smallest/imaginary frequencies is: $-780.8906 \mathrm{~cm}(-1)$

Zero-point correction=

Thermal correction to Energy=

Thermal correction to Enthalpy=

0.770927

0.817631

0.818654

Thermal correction to Gibbs Free Energy $=\quad 0.696150$

Sum of electronic and zero-point Energies = $\quad-1923.629671$

Sum of electronic and thermal Energies $=\quad-1923.582967$

Sum of electronic and thermal Enthalpies= $\quad-1923.581944$

Sum of electronic and thermal Free Energies $=\quad-1923.704448$

Cartesian Coordinates

$\begin{array}{cccccrrr}6 & -0.209388 & 1.849448 & -0.048437 & 6 & 2.085418 & 2.052645 & -0.977303 \\ 15 & -2.384535 & 0.002135 & 0.166245 & 6 & 2.940005 & 0.899441 & 1.633228 \\ 15 & 2.260876 & 0.425983 & -0.094714 & 6 & 3.694264 & -0.362772 & -1.103592 \\ 6 & -2.382739 & 1.586262 & 1.123322 & 6 & -1.346679 & 2.474720 & 0.501155 \\ 6 & -3.423051 & 0.461665 & -1.391002 & 6 & -1.492827 & 3.862567 & 0.497958 \\ 6 & -3.383166 & -1.178280 & 1.286778 & 6 & -0.494314 & 4.672274 & -0.035865\end{array}$




\begin{tabular}{|c|c|c|c|c|c|c|c|}
\hline 6 & 0.804958 & 2.692717 & -0.533576 & 1 & -2.305634 & -0.521541 & 3.094140 \\
\hline 6 & 0.660923 & 4.082313 & -0.537556 & 1 & -3.072946 & -2.109798 & 3.200636 \\
\hline 1 & -2.391835 & 4.311139 & 0.923871 & 1 & -1.565721 & -1.902811 & 2.274395 \\
\hline 1 & -0.608718 & 5.754485 & -0.044355 & 6 & 2.950991 & -0.337265 & 2.531667 \\
\hline 1 & 1.467245 & 4.706323 & -0.926067 & 1 & 3.334359 & -0.055641 & 3.523093 \\
\hline 1 & -3.369422 & 2.057484 & 1.194536 & 1 & 1.935967 & -0.729354 & 2.663711 \\
\hline 1 & -2.072600 & 1.317518 & 2.144181 & 1 & 3.586142 & -1.145356 & 2.152876 \\
\hline 1 & 2.952258 & 2.711645 & -0.829746 & 6 & 4.433745 & -1.449997 & -0.322047 \\
\hline 1 & 2.048455 & 1.800797 & -2.049013 & 1 & 5.076335 & -2.011389 & -1.015902 \\
\hline 6 & 4.339811 & 1.514040 & 1.594081 & 1 & 5.087013 & -1.038869 & 0.455347 \\
\hline 1 & 5.114601 & 0.810440 & 1.273470 & 1 & 3.758415 & -2.171983 & 0.151957 \\
\hline 1 & 4.388439 & 2.404858 & 0.955188 & 6 & -4.124736 & -0.756265 & -1.992235 \\
\hline 1 & 4.605863 & 1.837484 & 2.610601 & 1 & -4.505989 & -0.486360 & -2.987477 \\
\hline 6 & 4.718484 & 0.666548 & -1.595884 & 1 & -3.455017 & -1.615690 & -2.123579 \\
\hline 1 & 4.287100 & 1.367863 & -2.318802 & 1 & -4.987500 & -1.077513 & -1.398381 \\
\hline 1 & 5.184246 & 1.246058 & -0.795010 & 6 & -3.666410 & -2.534552 & 0.631570 \\
\hline 1 & 5.523938 & 0.128111 & -2.116405 & 1 & -4.483746 & -2.491742 & -0.092313 \\
\hline 6 & -4.476807 & 1.529474 & -1.083999 & 1 & -2.794110 & -2.963763 & 0.127329 \\
\hline 1 & -5.150733 & 1.258191 & -0.264179 & 1 & -3.969488 & -3.244652 & 1.414012 \\
\hline 1 & -4.020044 & 2.498400 & -0.850147 & 77 & -0.037061 & -0.266132 & -0.097738 \\
\hline 1 & -5.097506 & 1.674703 & -1.979979 & 6 & -0.221875 & -1.921704 & -1.641752 \\
\hline 6 & -4.715136 & -0.575444 & 1.733601 & 1 & -0.052441 & -0.398018 & -1.823895 \\
\hline 1 & -4.595150 & 0.384586 & 2.249838 & 6 & -0.037230 & -2.477790 & -0.322704 \\
\hline 1 & -5.412575 & -0.436045 & 0.899319 & 1 & 0.012195 & -0.193288 & 1.539030 \\
\hline 1 & -5.197812 & -1.265553 & 2.441051 & 1 & -0.943258 & -2.874423 & 0.143761 \\
\hline 6 & 2.018503 & 1.931626 & 2.291974 & 1 & -1.214725 & -1.970545 & -2.095592 \\
\hline 1 & 2.015597 & 2.894455 & 1.768063 & 1 & 0.558089 & -2.108212 & -2.383258 \\
\hline 1 & 0.984903 & 1.576978 & 2.363464 & 6 & 1.305994 & -3.626252 & 1.455184 \\
\hline 1 & 2.386641 & 2.110237 & 3.312820 & 1 & 1.412778 & -2.680706 & 1.998826 \\
\hline 6 & 3.078721 & -1.003068 & -2.350189 & 6 & 0.966813 & -4.728188 & -0.757046 \\
\hline 1 & 2.421206 & -0.317830 & -2.900551 & 1 & 1.805352 & -5.409394 & -0.556673 \\
\hline 1 & 3.887029 & -1.302686 & -3.032719 & 6 & 1.137668 & -3.390222 & -0.037496 \\
\hline 1 & 2.508373 & -1.904215 & -2.107180 & 1 & 2.053750 & -2.919837 & -0.422557 \\
\hline 6 & -2.495470 & 1.038624 & -2.464630 & 1 & 0.893385 & -4.597108 & -1.844744 \\
\hline 1 & -1.915476 & 1.895664 & -2.105303 & 1 & 0.046675 & -5.229291 & -0.421962 \\
\hline 1 & -1.789943 & 0.293323 & -2.846556 & 1 & 2.178985 & -4.256929 & 1.672538 \\
\hline 1 & -3.115257 & 1.377913 & -3.307604 & 1 & 0.422248 & -4.142100 & 1.860678 \\
\hline 6 & -2.523364 & -1.435171 & 2.528807 & & & & \\
\hline
\end{tabular}

6

The smallest/imaginary frequencies is: $30.3467 \mathrm{~cm}(-1)$

$\begin{array}{lc}\text { Zero-point correction }= & 0.771233 \\ \text { Thermal correction to Energy= } & 0.818607 \\ \text { Thermal correction to Enthalpy= } & 0.819630 \\ \text { Thermal correction to Gibbs Free Energy= } & 0.695517 \\ \text { Sum of electronic and zero-point Energies= } & -1923.651794 \\ \text { Sum of electronic and thermal Energies= } & -1923.604421 \\ \text { Sum of electronic and thermal Enthalpies }= & -1923.603398 \\ \text { Sum of electronic and thermal Free Energies }= & -1923.727511\end{array}$


Cartesian Coordinates

$\begin{array}{cccc}\cdots & -0.015649 & 1.856187 & -0.405909 \\ 15 & -2.336106 & 0.228002 & 0.211366 \\ 15 & 2.352275 & 0.250585 & 0.008240 \\ 6 & -2.314007 & 2.045979 & 0.542366 \\ 6 & -3.322276 & 0.096926 & -1.431430 \\ 6 & -3.403830 & -0.448495 & 1.651910 \\ 6 & 2.371026 & 1.705255 & -1.137347 \\ 6 & 3.119573 & 0.903171 & 1.631192 \\ 6 & 3.588045 & -0.913679 & -0.868934 \\ 6 & -1.163632 & 2.649506 & -0.206210 \\ 6 & -1.217200 & 3.977192 & -0.632832 \\ 6 & -0.111237 & 4.567331 & -1.235384 \\ 6 & 1.100969 & 2.487460 & -0.981083 \\ 6 & 1.051779 & 3.823096 & -1.388969 \\ 1 & -2.129157 & 4.554758 & -0.475572 \\ 1 & -0.150917 & 5.603074 & -1.566788 \\ 1 & 1.937380 & 4.279611 & -1.833279 \\ 1 & -3.270453 & 2.541364 & 0.338495 \\ 1 & -2.119388 & 2.145765 & 1.619338 \\ 1 & 3.263974 & 2.330937 & -0.997834 \\ 1 & 2.433259 & 1.284351 & -2.151788 \\ 6 & 4.619149 & 1.183136 & 1.553860 \\ 1 & 5.215143 & 0.266473 & 1.486686 \\ 1 & 4.889558 & 1.842085 & 0.719426 \\ 1 & 4.925366 & 1.693539 & 2.478367 \\ 6 & 4.906439 & -0.253987 & -1.290237 \\ 1 & 4.754413 & 0.675209 & -1.851930 \\ 1 & 5.579364 & -0.045692 & -0.456212 \\ 1 & 5.433738 & -0.948032 & -1.961034 \\ 6 & -4.456044 & 1.122363 & -1.530280 \\ 1 & -5.166436 & 1.089699 & -0.699716 \\ 1 & -4.068577 & 2.145230 & -1.614156 \\ 1 & -5.023874 & 0.922574 & -2.450285 \\ 6 & -4.840854 & 0.073762 & 1.627724 \\ 1 & -4.892288 & 1.168352 & 1.575188 \\ 1 & -5.430376 & -0.344767 & 0.804130 \\ 1 & -5.338305 & -0.227102 & 2.561405 \\ 6 & 2.423574 & 2.218460 & 1.993931 \\ 1 & 2.651204 & 3.022901 & 1.284025 \\ 1 & 1.335618 & 2.108428 & 2.054672 \\ 1 & 2.786707 & 2.539923 & 2.980740 \\ 6 & 2.919656 & -1.388952 & -2.165456 \\ 1 & 2.955403 & -0.615296 & -2.942323 \\ 1 & 3.466872 & -2.260058 & -2.553598\end{array}$

$\begin{array}{lrrr}1 & 1.871813 & -1.663983 & -2.034752 \\ 6 & -2.377762 & 0.375148 & -2.603548 \\ 1 & -1.873913 & 1.345246 & -2.515868 \\ 1 & -1.604702 & -0.392699 & -2.698405 \\ 1 & -2.972825 & 0.386398 & -3.528405 \\ 6 & -2.767411 & 0.003191 & 2.971332 \\ 1 & -2.916897 & 1.071603 & 3.165068 \\ 1 & -3.250656 & -0.540842 & 3.795137 \\ 1 & -1.690893 & -0.199419 & 3.010800 \\ 6 & 2.863862 & -0.098530 & 2.757887 \\ 1 & 3.282601 & 0.300809 & 3.692906 \\ 1 & 1.790709 & -0.261212 & 2.908976 \\ 1 & 3.340977 & -1.069806 & 2.577271 \\ 6 & 3.909204 & -2.108568 & 0.028923 \\ 1 & 4.511488 & -2.837441 & -0.532380 \\ 1 & 4.497442 & -1.812945 & 0.906359 \\ 1 & 3.012647 & -2.629843 & 0.385981 \\ 6 & -3.887969 & -1.310570 & -1.615493 \\ 1 & -4.282024 & -1.405282 & -2.637528 \\ 1 & -3.116679 & -2.083060 & -1.494157 \\ 1 & -4.715612 & -1.530046 & -0.931121 \\ 6 & -3.442013 & -1.979189 & 1.666828 \\ 1 & -3.830155 & -2.416197 & 0.742094 \\ 1 & -2.457456 & -2.414678 & 1.864696 \\ 1 & -4.106644 & -2.302928 & 2.480718 \\ 77 & 0.011813 & -0.181414 & 0.155641 \\ 6 & -0.339824 & -2.429751 & 0.127525 \\ 1 & 0.069307 & -0.453506 & -1.489629 \\ 6 & -0.184911 & -3.396467 & -2.196319 \\ 1 & -0.126357 & -2.401806 & -2.654233 \\ 6 & 0.249504 & -4.803224 & -0.182288 \\ 1 & 0.712144 & -4.856915 & 0.811177 \\ 6 & 0.164971 & -2.036979 & 1.377278 \\ 1 & -0.009080 & 0.459251 & 1.706590 \\ 1 & -0.479184 & -1.943876 & 2.249940 \\ 1 & 1.198899 & -2.285985 & 1.624415 \\ 1 & -1.424127 & -2.554793 & 0.049698 \\ 1 & -0.807778 & -5.085295 & -0.071770 \\ 1 & 0.727310 & -5.556053 & -0.822940 \\ 1 & 0.349565 & -4.106923 & -2.840086 \\ 1 & 1.243769 & -3.696834 & -2.187647 \\ 1 & & -3.401808 & -0.782725 \\ 1 & & -3.151970 \\ 1 & & & -817209\end{array}$

alkene

The smallest/imaginary frequencies is: $115.3212 \mathrm{~cm}(-1)$ 

Zero-point correction=
0.136040
Thermal correction to Energy=
0.143377
Thermal correction to Enthalpy=
0.144401
Thermal correction to Gibbs Free Energy=
Sum of electronic and zero-point Energies=
Sum of electronic and thermal Energies=
0.103879
$-196.237233$
$-196.229896$
$-196.228873$
$-196.269394$

\begin{tabular}{crrr}
\multicolumn{4}{c}{ Cartesian Coordinates } \\
$\ldots \ldots \ldots \ldots \ldots \ldots \ldots \ldots \ldots \ldots \ldots \ldots \ldots \ldots \ldots \ldots \ldots \ldots \ldots \ldots \ldots \ldots$ \\
6 & -1.969339 & -0.051168 & -0.240797 \\
6 & -0.885733 & -0.632261 & 0.267569 \\
6 & 0.550763 & 1.431507 & -0.014968 \\
1 & 0.328982 & 1.523187 & -1.087055 \\
6 & 1.506871 & -0.866408 & -0.314550 \\
1 & 1.307841 & -0.847742 & -1.394491 \\
1 & -2.909769 & -0.590582 & -0.331020 \\
1 & -1.971927 & 0.981591 & -0.585917
\end{tabular}

Geometries from Cyclooctane dehydrogenation

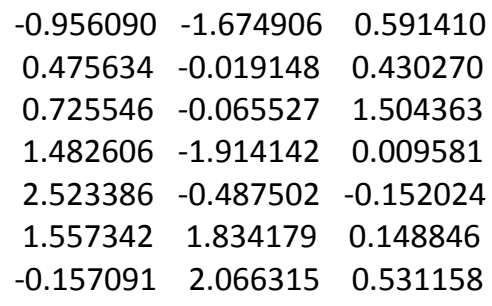

\section{1}

The smallest/imaginary frequencies is: $133.0573 \mathrm{~cm}(-1)$

$\begin{array}{lc}\text { Zero-point correction= } & 0.226862 \\ \text { Thermal correction to Energy= } & 0.236324 \\ \text { Thermal correction to Enthalpy= } & 0.237348 \\ \text { Thermal correction to Gibbs Free Energy= } & 0.191383 \\ \text { Sum of electronic and zero-point Energies }= & -314.017633 \\ \text { Sum of electronic and thermal Energies }= & -314.008170 \\ \text { Sum of electronic and thermal Enthalpies= } & -314.007147 \\ \text { Sum of electronic and thermal Free Energies= } & -314.053111\end{array}$

Cartesian Coordinates

\begin{tabular}{|c|c|c|c|c|c|c|c|}
\hline 6 & 1.660752 & 0.608376 & 0.476550 & 1 & 1.132377 & 2.625902 & 0.023802 \\
\hline 6 & 0.740257 & 1.624962 & -0.204168 & 1 & -0.788721 & -2.521527 & 0.839050 \\
\hline 6 & -0.527000 & -1.493160 & 0.557490 & 1 & -1.132336 & 2.625918 & -0.023811 \\
\hline 6 & -0.740231 & 1.624973 & 0.204164 & 1 & -2.419792 & -1.388916 & -0.467874 \\
\hline 6 & -1.815967 & -0.754099 & 0.198660 & 1 & -2.667903 & 1.041888 & -0.552798 \\
\hline 6 & -1.660737 & 0.608397 & -0.476558 & 1 & -1.329683 & 0.480562 & -1.518588 \\
\hline 1 & 1.329712 & 0.480557 & 1.518587 & 6 & 1.815952 & -0.754127 & -0.198662 \\
\hline 1 & 0.815464 & 1.526111 & -1.299953 & 1 & 2.419778 & -1.388947 & 0.467868 \\
\hline 1 & -0.815441 & 1.526128 & 1.299949 & 1 & 2.414278 & -0.629707 & -1.114054 \\
\hline 1 & -0.099609 & -1.050712 & 1.468387 & 6 & 0.526973 & -1.493178 & -0.557474 \\
\hline 1 & -2.414303 & -0.629667 & 1.114045 & 1 & 0.099585 & -1.050747 & -1.468381 \\
\hline 1 & 2.667927 & 1.041850 & 0.552772 & 1 & 0.788681 & -2.521555 & -0.839011 \\
\hline
\end{tabular}


The smallest/imaginary frequencies is: $-559.4071 \mathrm{~cm}(-1)$

$\begin{array}{lc}\text { Zero-point correction }= & 0.840138 \\ \text { Thermal correction to Energy= } & 0.889608 \\ \text { Thermal correction to Enthalpy= } & 0.890631 \\ \text { Thermal correction to Gibbs Free Energy= } & 0.760339 \\ \text { Sum of electronic and zero-point Energies }= & -2040.209639 \\ \text { Sum of electronic and thermal Energies }= & -2040.160169 \\ \text { Sum of electronic and thermal Enthalpies }= & -2040.159146 \\ \text { Sum of electronic and thermal Free Energies }= & -2040.289438\end{array}$

Cartesian Coordinates

\begin{tabular}{|c|c|c|c|c|c|c|c|}
\hline 6 & -1.432258 & -1.724151 & -0.533647 & 1 & 0.752921 & -3.537229 & -2.302364 \\
\hline 15 & -2.219055 & 0.988587 & -0.047443 & 1 & 0.663986 & -1.798454 & -2.633403 \\
\hline 15 & 1.363720 & -1.936948 & 0.184668 & 1 & 1.898381 & -2.796622 & -3.432665 \\
\hline 6 & -3.123740 & -0.055439 & -1.293325 & 6 & 1.649657 & -1.554326 & 2.910617 \\
\hline 6 & -3.225247 & 0.737551 & 1.568514 & 1 & 0.685262 & -2.063661 & 3.035485 \\
\hline 6 & -2.557279 & 2.746248 & -0.731721 & 1 & 2.220564 & -1.694343 & 3.840259 \\
\hline 6 & 0.219351 & -3.386224 & 0.363804 & 1 & 1.442542 & -0.484194 & 2.794202 \\
\hline 6 & 2.367916 & -2.362020 & -1.376806 & 6 & -2.736541 & -0.535272 & 2.269100 \\
\hline 6 & 2.460577 & -2.111029 & 1.737217 & 1 & -2.917914 & -1.436275 & 1.672021 \\
\hline 6 & -2.720220 & -1.481665 & -1.064336 & 1 & -1.662092 & -0.493703 & 2.486516 \\
\hline 6 & -3.609836 & -2.520973 & -1.341832 & 1 & -3.285755 & -0.640457 & 3.217267 \\
\hline 6 & -3.237660 & -3.841704 & -1.113155 & 6 & -2.073315 & 2.753078 & -2.187959 \\
\hline 6 & -1.094401 & -3.074680 & -0.293926 & 1 & -2.785443 & 2.266310 & -2.864021 \\
\hline 6 & -1.979031 & -4.114576 & -0.589187 & 1 & -1.970761 & 3.793949 & -2.526808 \\
\hline 1 & -4.599297 & -2.293470 & -1.741064 & 1 & -1.097188 & 2.267359 & -2.310451 \\
\hline 1 & -3.928206 & -4.653463 & -1.334535 & 6 & 3.194463 & -1.148571 & -1.797166 \\
\hline 1 & -1.684337 & -5.145881 & -0.389600 & 1 & 3.725712 & -1.376269 & -2.732960 \\
\hline 1 & -4.212353 & 0.087216 & -1.280684 & 1 & 2.551544 & -0.280105 & -1.981290 \\
\hline 1 & -2.768343 & 0.276615 & -2.277580 & 1 & 3.948799 & -0.865564 & -1.053576 \\
\hline 1 & 0.663957 & -4.326861 & 0.007303 & 6 & 3.747504 & -1.296158 & 1.617848 \\
\hline 1 & 0.057145 & -3.510938 & 1.444929 & 1 & 4.277193 & -1.319952 & 2.581370 \\
\hline 6 & 3.282551 & -3.576258 & -1.243194 & 1 & 4.434064 & -1.700123 & 0.864454 \\
\hline 1 & 4.128018 & -3.393727 & -0.569731 & 1 & 3.555320 & -0.241587 & 1.380899 \\
\hline 1 & 2.750071 & -4.472127 & -0.899389 & 6 & -2.973336 & 1.905390 & 2.521802 \\
\hline 1 & 3.706037 & -3.813095 & -2.230424 & 1 & -3.408981 & 1.667421 & 3.502905 \\
\hline 6 & 2.827705 & -3.560332 & 2.068803 & 1 & -1.900007 & 2.082507 & 2.676938 \\
\hline 1 & 1.947474 & -4.186531 & 2.253283 & 1 & -3.437244 & 2.839093 & 2.183880 \\
\hline 1 & 3.430476 & -4.041504 & 1.293690 & 6 & -1.760844 & 3.809033 & 0.025201 \\
\hline 1 & 3.423783 & -3.568296 & 2.993038 & 77 & -0.134876 & -0.155485 & 0.00997 \\
\hline 6 & -4.727649 & 0.576070 & 1.335386 & 6 & 1.459578 & 1.536613 & 0.228118 \\
\hline 1 & -5.189535 & 1.427208 & 0.826192 & 6 & 1.452186 & 2.116602 & -1.194436 \\
\hline 1 & -4.953661 & -0.333153 & 0.764436 & 6 & 1.422064 & 2.558420 & 1.379562 \\
\hline 1 & -5.226432 & 0.471943 & 2.310158 & 6 & 2.814774 & 2.490929 & -1.823072 \\
\hline 6 & -4.032048 & 3.145955 & -0.728333 & 1 & 0.998039 & 1.367650 & -1.864356 \\
\hline 1 & -4.673243 & 2.402131 & -1.217386 & 1 & 1.691472 & 2.018327 & 2.305131 \\
\hline 1 & -4.418211 & 3.329517 & 0.280881 & 1 & 0.389096 & 2.900357 & 1.540412 \\
\hline 1 & -4.144430 & 4.086078 & -1.289019 & 6 & 2.323592 & 3.786792 & 1.266761 \\
\hline 6 & 1.354016 & -2.639319 & -2.490102 & 6 & 3.347368 & 3.905331 & -1.574453 \\
\hline
\end{tabular}




$\begin{array}{rrrrrrrrr}1 & 3.575486 & 1.749892 & -1.527596 & 1 & 4.447940 & 3.782659 & 1.745272 \\ 1 & 2.699177 & 2.367945 & -2.909786 & 1 & 5.258623 & 3.645944 & -0.617804 \\ 6 & 3.784365 & 3.459285 & 0.932469 & 1 & 4.449814 & 5.154528 & -0.225218 \\ 1 & 1.910231 & 4.472033 & 0.512467 & 1 & 2.387325 & 0.960783 & 0.327858 \\ 1 & 2.261714 & 4.339066 & 2.214384 & 1 & 0.526564 & 0.678495 & 1.200628 \\ 6 & 4.276131 & 4.077621 & -0.373198 & 1 & -2.003570 & 3.857455 & 1.091131 \\ 1 & 3.900268 & 4.238384 & -2.464065 & 1 & -0.682152 & 3.651406 & -0.067559 \\ 1 & 2.493486 & 4.595643 & -1.498095 & 1 & -1.986354 & 4.795585 & -0.405518 \\ 1 & 3.909513 & 2.367283 & 0.877421 & 1 & 0.786605 & 2.991273 & -1.256432\end{array}$

5

The smallest/imaginary frequencies is: $25.5296 \mathrm{~cm}(-1)$

$\begin{array}{lc}\text { Zero-point correction }= & 0.841140 \\ \text { Thermal correction to Energy= } & 0.890784 \\ \text { Thermal correction to Enthalpy= } & 0.891807 \\ \text { Thermal correction to Gibbs Free Energy= } & 0.762221 \\ \text { Sum of electronic and zero-point Energies }= & -2040.216586 \\ \text { Sum of electronic and thermal Energies }= & -2040.166942 \\ \text { Sum of electronic and thermal Enthalpies }= & -2040.165919 \\ \text { Sum of electronic and thermal Free Energies }= & -2040.295505\end{array}$

Cartesian Coordinates

$\begin{array}{lrrr}1 & 3.813470 & -3.697642 & 1.246295 \\ 1 & 3.799628 & -3.243619 & 2.950381 \\ 6 & -4.756303 & 0.073291 & 1.309478 \\ 1 & -5.293192 & 0.880304 & 0.803530 \\ 1 & -4.881594 & -0.846378 & 0.724559 \\ 1 & -5.255560 & -0.092317 & 2.275150 \\ 6 & -4.330545 & 2.748619 & -0.661813 \\ 1 & -4.902743 & 1.957540 & -1.162234 \\ 1 & -4.722150 & 2.873613 & 0.354242 \\ 1 & -4.537568 & 3.685017 & -1.201209 \\ 6 & 1.604124 & -2.433781 & -2.499837 \\ 1 & 1.080703 & -3.380782 & -2.322392 \\ 1 & 0.843241 & -1.655795 & -2.641920 \\ 1 & 2.160526 & -2.533397 & -3.443043 \\ 6 & 1.829174 & -1.416405 & 2.928259 \\ 1 & 0.903164 & -1.996648 & 3.035834 \\ 1 & 2.411759 & -1.551325 & 3.850918 \\ 1 & 1.555977 & -0.357912 & 2.853479 \\ 6 & -2.688251 & -0.857457 & 2.257369 \\ 1 & -2.744050 & -1.754299 & 1.630552 \\ 1 & -1.639811 & -0.711764 & 2.540273 \\ 1 & -3.268033 & -1.048104 & 3.172999 \\ 6 & -2.365789 & 2.570445 & -2.150297 \\ 1 & -3.036561 & 2.025757 & -2.824572 \\ 1 & -2.372859 & 3.621597 & -2.471944 \\ 1 & -1.346781 & 2.191064 & -2.296167 \\ 6 & 3.304828 & -0.792553 & -1.787285\end{array}$




$\begin{array}{cccccccc}1 & 3.852990 & -0.962156 & -2.725478 & 6 & 1.878517 & 3.865557 & 1.321147 \\ 1 & 2.589474 & 0.018868 & -1.962283 & 6 & 2.843154 & 4.271755 & -1.533701 \\ 1 & 4.030233 & -0.449670 & -1.040845 & 1 & 3.358415 & 2.169230 & -1.643730 \\ 6 & 3.870825 & -0.925885 & 1.616179 & 1 & 2.381018 & 2.759696 & -2.973474 \\ 1 & 4.402243 & -0.897170 & 2.578475 & 6 & 3.361192 & 3.740749 & 0.947667 \\ 1 & 4.593742 & -1.255044 & 0.860219 & 1 & 1.376122 & 4.552261 & 0.622929 \\ 1 & 3.564574 & 0.101754 & 1.381687 & 1 & 1.772300 & 4.339676 & 2.307401 \\ 6 & -3.157545 & 1.546682 & 2.548624 & 6 & 3.753255 & 4.487176 & -0.324982 \\ 1 & -3.583103 & 1.247732 & 3.517283 & 1 & 3.336854 & 4.738467 & -2.398098 \\ 1 & -2.109692 & 1.825189 & 2.725125 & 1 & 1.904560 & 4.833183 & -1.407506 \\ 1 & -3.704942 & 2.437406 & 2.219990 & 1 & 3.614692 & 2.677104 & 0.825328 \\ 6 & -2.123039 & 3.615226 & 0.078884 & 1 & 3.997895 & 4.097593 & 1.768419 \\ 77 & -0.083410 & -0.135912 & 0.018544 & 1 & 4.779809 & 4.198261 & -0.598914 \\ 6 & 1.283606 & 1.597308 & 0.137993 & 1 & 3.791816 & 5.567695 & -0.116451 \\ 6 & 1.215219 & 2.237673 & -1.251778 & 1 & 2.288635 & 1.154922 & 0.211325 \\ 6 & 1.124940 & 2.530573 & 1.343893 & 1 & 0.035259 & 0.171964 & 1.532649 \\ 6 & 2.500616 & 2.819894 & -1.881304 & 1 & -2.353969 & 3.619447 & 1.148737 \\ 1 & 0.860042 & 1.455825 & -1.957718 & 1 & -1.035668 & 3.562150 & -0.028656 \\ 1 & 1.457545 & 1.965051 & 2.232849 & 1 & -2.449533 & 4.582725 & -0.329556 \\ 1 & 0.062541 & 2.746105 & 1.529304 & 1 & 0.434200 & 3.014529 & -1.292603\end{array}$

TS2

The smallest/imaginary frequencies is: $-750.4960 \mathrm{~cm}(-1)$

$\begin{array}{lc}\text { Zero-point correction }= & 0.838378 \\ \text { Thermal correction to Energy= } & 0.887018 \\ \text { Thermal correction to Enthalpy= } & 0.888041 \\ \text { Thermal correction to Gibbs Free Energy= } & 0.761327 \\ \text { Sum of electronic and zero-point Energies }= & -2040.200142 \\ \text { Sum of electronic and thermal Energies }= & -2040.151503 \\ \text { Sum of electronic and thermal Enthalpies }= & -2040.150479 \\ \text { Sum of electronic and thermal Free Energies }= & -2040.277193\end{array}$

Cartesian Coordinates

$\begin{array}{cccccccc}6 & 0.152207 & -2.142327 & -0.321705 & 1 & 2.148854 & -4.865398 & 0.293920 \\ 15 & -2.284430 & -0.627134 & -0.039380 & 1 & -3.065203 & -2.650066 & -1.293093 \\ 15 & 2.408283 & -0.440931 & 0.131998 & 1 & -1.965961 & -1.596595 & -2.202437 \\ 6 & -2.146776 & -2.054219 & -1.219978 & 1 & 3.397122 & -2.699235 & 0.434088 \\ 6 & -2.993626 & -1.437978 & 1.561055 & 1 & 2.359533 & -2.186329 & 1.768602 \\ 6 & -3.722815 & 0.332986 & -0.898697 & 6 & 4.846469 & -0.810706 & -1.375846 \\ 6 & 2.443564 & -2.206928 & 0.670990 & 1 & 5.419592 & 0.002478 & -0.916153 \\ 6 & 3.362670 & -0.476616 & -1.528344 & 1 & 5.020568 & -1.730072 & -0.803550 \\ 6 & 3.470562 & 0.412781 & 1.471800 & 1 & 5.270656 & -0.972481 & -2.377359 \\ 6 & -0.952561 & -2.858200 & -0.823070 & 6 & 4.706671 & -0.396824 & 1.879747 \\ 6 & -0.952456 & -4.250484 & -0.924483 & 1 & 4.446751 & -1.385712 & 2.274467 \\ 6 & 0.161726 & -4.984054 & -0.530212 & 1 & 5.431066 & -0.530335 & 1.073321 \\ 6 & 1.259895 & -2.915594 & 0.081442 & 1 & 5.220895 & 0.143176 & 2.687954 \\ 6 & 1.267383 & -4.307771 & -0.027011 & 6 & -3.903974 & -2.632669 & 1.261354 \\ 1 & -1.834129 & -4.761101 & -1.315306 & 1 & -4.745047 & -2.402704 & 0.603270 \\ 1 & 0.166551 & -6.069315 & -0.610649 & 1 & -3.339835 & -3.465431 & 0.824062\end{array}$




$\begin{array}{lrrr}1 & -4.326205 & -2.995017 & 2.209814 \\ 6 & -5.039497 & -0.446637 & -0.951428 \\ 1 & -4.926767 & -1.453854 & -1.368387 \\ 1 & -5.528959 & -0.523799 & 0.025718 \\ 1 & -5.731668 & 0.096035 & -1.612312 \\ 6 & 2.727158 & -1.556516 & -2.409737 \\ 1 & 2.887434 & -2.568863 & -2.021926 \\ 1 & 1.648349 & -1.406360 & -2.530119 \\ 1 & 3.192161 & -1.506420 & -3.405061 \\ 6 & 2.611789 & 0.559270 & 2.733481 \\ 1 & 2.284102 & -0.411894 & 3.124271 \\ 1 & 3.221105 & 1.036841 & 3.514372 \\ 1 & 1.715261 & 1.166207 & 2.578706 \\ 6 & -1.866165 & -1.968671 & 2.451158 \\ 1 & -1.209264 & -2.675458 & 1.930869 \\ 1 & -1.245867 & -1.160794 & 2.851416 \\ 1 & -2.326204 & -2.495941 & 3.299913 \\ 6 & -3.291931 & 0.583935 & -2.350937 \\ 1 & -3.439476 & -0.300649 & -2.980762 \\ 1 & -3.911322 & 1.387473 & -2.775014 \\ 1 & -2.243028 & 0.888593 & -2.440597 \\ 6 & 3.234604 & 0.850219 & -2.277622 \\ 1 & 3.850119 & 0.802690 & -3.187438 \\ 1 & 2.201466 & 1.027819 & -2.597032 \\ 1 & 3.579409 & 1.714468 & -1.698104 \\ 6 & 3.924718 & 1.798287 & 1.008804 \\ 1 & 4.402747 & 2.320570 & 1.850057 \\ 1 & 4.666229 & 1.743254 & 0.202761 \\ 1 & 3.095144 & 2.429396 & 0.664809 \\ 6 & -3.753287 & -0.396121 & 2.382582 \\ 1 & -4.000216 & -0.826564 & 3.363778\end{array}$

$\begin{array}{cccc}1 & -3.144446 & 0.500101 & 2.566079 \\ 1 & -4.698350 & -0.088445 & 1.921419 \\ 6 & -4.016446 & 1.686541 & -0.250163 \\ 77 & 0.095859 & -0.037575 & 0.033181 \\ 6 & 0.197727 & 2.099081 & 0.563392 \\ 6 & 0.294506 & 2.131250 & -0.878836 \\ 6 & -0.892856 & 2.801138 & 1.333667 \\ 1 & -1.154958 & 2.222075 & 2.232666 \\ 1 & -1.813245 & 2.853479 & 0.742747 \\ 6 & -0.478299 & 4.207968 & 1.771588 \\ 6 & -0.353684 & 4.421103 & -1.800924 \\ 6 & 0.113735 & 5.081007 & 0.669237 \\ 1 & -1.347949 & 4.720452 & 2.212295 \\ 1 & 0.265171 & 4.122435 & 2.580301 \\ 6 & -0.760411 & 5.249543 & -0.581262 \\ 1 & -0.874941 & 4.830290 & -2.678254 \\ 1 & 1.094477 & 4.678529 & 0.369785 \\ 1 & 0.325146 & 6.068401 & 1.102176 \\ 1 & -0.737063 & 6.303894 & -0.888767 \\ 1 & -1.815509 & 5.043565 & -0.335289 \\ 1 & 1.160776 & 2.249421 & 1.054906 \\ 1 & 0.136393 & -0.519307 & 1.574231 \\ 1 & -4.198401 & 1.630506 & 0.826799 \\ 1 & -3.210837 & 2.405146 & -0.415337 \\ 1 & -4.918685 & 2.110646 & -0.714290 \\ 1 & 0.097576 & 0.791836 & -1.552031 \\ 6 & -0.647200 & 2.919426 & -1.768631 \\ 1 & -1.692464 & 2.761518 & -1.481145 \\ 1 & -0.558123 & 2.532117 & -2.794960 \\ 1 & 0.720508 & 4.576384 & -1.995782 \\ 1 & 1.319636 & 2.249263 & -1.241068\end{array}$

6

(Structure could not be optimized in solvent phase even after multiple attempts)

Gas phase optimized geometry corrections added to total electronic energy from solvent phase single point energies:

Zero-point correction=

Thermal correction to Energy=

Thermal correction to Enthalpy=

Thermal correction to Gibbs Free Energy=

Total Electronic Energy from single point energy calculation in solvent $(p-x y l e n e)=-2041.051486$
0.839796

0.888920

0.889944

\section{Cartesian Coordinates}

$\begin{array}{cccc}77 & 0.133234 & 0.061324 & -0.187172 \\ 6 & 0.777114 & 2.051647 & -0.591352 \\ 15 & -1.985037 & 1.216256 & -0.077315 \\ 15 & 2.482106 & -0.252364 & -0.029551 \\ 6 & -3.551333 & 0.710797 & -1.055799 \\ 6 & -2.491450 & 1.847699 & 1.662221 \\ 6 & 3.186941 & -0.180306 & 1.752255\end{array}$

$\begin{array}{lrrr}6 & 3.389155 & -1.597105 & -1.044955 \\ 6 & -0.124583 & 3.115586 & -0.781054 \\ 6 & 0.304415 & 4.437613 & -0.910254 \\ 6 & 1.657740 & 4.746216 & -0.902001 \\ 6 & 2.142823 & 2.384929 & -0.696849 \\ 6 & 2.574054 & 3.706900 & -0.821956 \\ 1 & -0.437333 & 5.229286 & -1.024626\end{array}$




$$
\begin{array}{rrr}
1.993191 & 5.777820 & -0.982503 \\
3.643770 & 3.915485 & -0.868649 \\
4.696061 & -0.410754 & 1.830103 \\
4.971904 & -1.452083 & 1.629845 \\
5.264452 & 0.239942 & 1.154717 \\
5.028457 & -0.183232 & 2.852712 \\
4.890013 & -1.343847 & -1.237148 \\
5.105106 & -0.338345 & -1.616287 \\
5.476379 & -1.501345 & -0.330288 \\
5.262292 & -2.054993 & -1.988318 \\
-4.529787 & 1.850652 & -1.364177 \\
-5.072933 & 2.209735 & -0.487746 \\
-4.045520 & 2.707506 & -1.844926 \\
-5.278856 & 1.469106 & -2.073065 \\
-3.805028 & 2.629392 & 1.680868 \\
-3.823189 & 3.456250 & 0.960876 \\
-4.678186 & 1.991367 & 1.505506 \\
-3.928864 & 3.070771 & 2.679902 \\
2.902591 & 1.223663 & 2.298384 \\
3.471032 & 2.003311 & 1.778727 \\
1.838766 & 1.475126 & 2.237640 \\
3.200083 & 1.248622 & 3.356092 \\
2.775457 & -1.594894 & -2.452716 \\
3.058107 & -0.694216 & -3.011287 \\
3.169819 & -2.457100 & -3.008544 \\
1.683665 & -1.637954 & -2.462888 \\
-3.053315 & 0.186518 & -2.406916 \\
-2.691579 & 1.001349 & -3.046006 \\
-2.234391 & -0.526921 & -2.306855 \\
-3.887320 & -0.297454 & -2.935065 \\
-1.389481 & 2.787986 & 2.162435 \\
-1.325872 & 3.713360 & 1.579100 \\
-1.619415 & 3.065625 & 3.200893 \\
-0.406291 & 2.306619 & 2.148246 \\
-0.243186 & -2.006941 & 0.720979 \\
-0.290874 & -2.110371 & -0.677149 \\
0.218777 & 0.431230 & 1.442219 \\
0.712204 & -2.259444 & 1.181868 \\
0.107827 & 0.032652 & -1.866987 \\
0.639997 & -2.413163 & -1.150813
\end{array}
$$

$\begin{array}{lrrr}6 & 3.158823 & 1.287297 & -0.777571 \\ 1 & 3.341934 & 1.057164 & -1.837590 \\ 1 & 4.130705 & 1.565051 & -0.346176 \\ 6 & -1.578800 & 2.802838 & -0.928212 \\ 1 & -2.229132 & 3.624605 & -0.597721 \\ 1 & -1.795272 & 2.637153 & -1.994061 \\ 6 & -2.893098 & -4.247359 & 0.794133 \\ 1 & -3.540874 & -4.963919 & 1.317896 \\ 1 & -3.543978 & -3.382730 & 0.593062 \\ 6 & -1.772553 & -3.798552 & 1.743626 \\ 1 & -0.876362 & -4.424408 & 1.605693 \\ 1 & -2.105668 & -3.988070 & 2.772779 \\ 6 & -1.395007 & -2.305261 & 1.642968 \\ 1 & -1.124788 & -1.941935 & 2.644381 \\ 1 & -2.288683 & -1.735377 & 1.360896 \\ 6 & -2.489661 & -4.903030 & -0.527648 \\ 1 & -3.392401 & -4.968030 & -1.153423 \\ 1 & -2.191502 & -5.944110 & -0.332823 \\ 6 & -1.369116 & -4.241024 & -1.328394 \\ 1 & -1.352739 & -4.695458 & -2.328092 \\ 1 & -0.401903 & -4.508071 & -0.875436 \\ 6 & -1.443845 & -2.702030 & -1.444626 \\ 1 & -2.417249 & -2.342964 & -1.091029 \\ 1 & -1.371627 & -2.402479 & -2.498383 \\ 6 & -2.602692 & 0.690385 & 2.649633 \\ 1 & -3.379890 & -0.031823 & 2.370684 \\ 1 & -1.648647 & 0.160674 & 2.737584 \\ 1 & -2.865390 & 1.089006 & 3.639900 \\ 6 & -4.317662 & -0.386328 & -0.319599 \\ 1 & -5.132228 & -0.757152 & -0.958009 \\ 1 & -3.684483 & -1.242529 & -0.064379 \\ 1 & -4.773469 & -0.018784 & 0.608067 \\ 6 & 2.496340 & -1.188563 & 2.670407 \\ 1 & 1.420816 & -0.989264 & 2.736971 \\ 1 & 2.647009 & -2.227379 & 2.350335 \\ 1 & 2.921048 & -1.095394 & 3.679954 \\ 6 & 3.216779 & -2.971806 & -0.393968 \\ 1 & 2.167578 & -3.250437 & -0.241144 \\ 1 & 3.670644 & -3.737252 & -1.039331 \\ 1 & 3.724434 & -3.026987 & 0.577109\end{array}$

COE

The smallest/imaginary frequencies is: $166.5932 \mathrm{~cm}(-1)$

$\begin{array}{lc}\text { Zero-point correction= } & 0.203139 \\ \text { Thermal correction to Energy= } & 0.212083 \\ \text { Thermal correction to Enthalpy= } & 0.213107 \\ \text { Thermal correction to Gibbs Free Energy= } & 0.168398 \\ \text { Sum of electronic and zero-point Energies }= & -312.818432 \\ \text { Sum of electronic and thermal Energies= } & -312.809488 \\ \text { Sum of electronic and thermal Enthalpies }= & -312.808464\end{array}$


Cartesian Coordinates

$\begin{array}{rrrr}6 & 1.855378 & -0.493743 & -0.056030 \\ 6 & 1.367403 & 0.829873 & -0.658255 \\ 6 & -1.708800 & -0.789103 & 0.047010 \\ 6 & 0.571285 & 1.637816 & 0.316231 \\ 6 & -1.650674 & 0.680509 & -0.389112 \\ 6 & -0.754652 & 1.550375 & 0.447468 \\ 1 & 2.645894 & -0.287988 & 0.681039 \\ 1 & 0.758530 & 0.613239 & -1.547438 \\ 1 & -1.936631 & -0.836772 & 1.123539 \\ 1 & -2.669479 & 1.088720 & -0.359295 \\ 1 & 2.330205 & -1.081495 & -0.856172\end{array}$

$\begin{array}{lrrr}1 & 2.236504 & 1.399400 & -1.013688 \\ 1 & -2.563489 & -1.257145 & -0.462706 \\ 1 & 1.127944 & 2.279945 & 1.001741 \\ 1 & -1.343010 & 0.718017 & -1.445472 \\ 1 & -1.239165 & 2.132473 & 1.233607 \\ 6 & 0.774808 & -1.337429 & 0.617254 \\ 1 & 0.459981 & -0.851556 & 1.551992 \\ 1 & 1.243938 & -2.283420 & 0.920569 \\ 6 & -0.467823 & -1.639951 & -0.233603 \\ 1 & -0.214959 & -1.576783 & -1.304877 \\ 1 & -0.757810 & -2.686720 & -0.068615\end{array}$

Geometries from $n$-butane dehydrogenation

1

The smallest/imaginary frequencies is: $116.9809 \mathrm{~cm}(-1)$

$\begin{array}{lc}\text { Zero-point correction= } & 0.131160 \\ \text { Thermal correction to Energy= } & 0.137788 \\ \text { Thermal correction to Enthalpy= } & 0.138811 \\ \text { Thermal correction to Gibbs Free Energy= } & 0.100184 \\ \text { Sum of electronic and zero-point Energies }= & -158.194109 \\ \text { Sum of electronic and thermal Energies }= & -158.187481 \\ \text { Sum of electronic and thermal Enthalpies= } & -158.186457 \\ \text { Sum of electronic and thermal Free Energies }= & -158.225085\end{array}$

Cartesian Coordinates

\begin{tabular}{|c|c|c|c|c|c|c|c|}
\hline 6 & 0.560183 & -0.515027 & -0.000001 & 1 & 0.447731 & -1.169937 & -0.877901 \\
\hline 1 & 0.447733 & -1.169941 & 0.877896 & 1 & -2.084152 & -0.753181 & -0.883867 \\
\hline 6 & -1.940366 & -0.117963 & 0.000000 & 1 & -2.738327 & 0.633291 & 0.000004 \\
\hline 1 & -2.084152 & -0.753189 & 0.883862 & 1 & 2.738327 & -0.633291 & -0.000003 \\
\hline 6 & 1.940366 & 0.117963 & -0.000001 & 6 & -0.560183 & 0.515027 & 0.000002 \\
\hline 1 & 2.084151 & 0.753186 & -0.883865 & 1 & -0.447732 & 1.169936 & 0.877903 \\
\hline 1 & 2.084153 & 0.753183 & 0.883864 & 1 & -0.447732 & 1.169942 & -0.877893 \\
\hline
\end{tabular}

TS1

The smallest/imaginary frequencies is: $-603.7008 \mathrm{~cm}(-1)$

Zero-point correction=

Thermal correction to Energy=
0.744252

0.790815 


$\begin{array}{lc}\text { Thermal correction to Enthalpy= } & 0.791838 \\ \text { Thermal correction to Gibbs Free Energy= } & 0.667020 \\ \text { Sum of electronic and zero-point Energies }= & -1884.392561 \\ \text { Sum of electronic and thermal Energies }= & -1884.345998 \\ \text { Sum of electronic and thermal Enthalpies }= & -1884.344974 \\ \text { Sum of electronic and thermal Free Energies }= & -1884.469793\end{array}$

Cartesian Coordinates 
The smallest/imaginary frequencies is: $29.4982 \mathrm{~cm}(-1)$

$\begin{array}{lc}\text { Zero-point correction }= & 0.745307 \\ \text { Thermal correction to Energy= } & 0.791933 \\ \text { Thermal correction to Enthalpy= } & 0.792956 \\ \text { Thermal correction to Gibbs Free Energy= } & 0.669129 \\ \text { Sum of electronic and zero-point Energies }= & -1884.402916 \\ \text { Sum of electronic and thermal Energies= } & -1884.356290 \\ \text { Sum of electronic and thermal Enthalpies= } & -1884.355266 \\ \text { Sum of electronic and thermal Free Energies= } & -1884.479093\end{array}$

Cartesian Coordinates

\begin{tabular}{|c|c|c|c|c|c|c|c|}
\hline 6 & -0.181566 & -1.962841 & -0.579427 & 1 & -2.919036 & -2.346212 & 1.927461 \\
\hline 15 & 2.261570 & -0.438005 & -0.028769 & 1 & -1.477399 & -1.444012 & 2.425793 \\
\hline 15 & -2.357318 & -0.082795 & $5-0.008068$ & 1 & -2.927014 & -1.441124 & 3.447476 \\
\hline 6 & 2.323969 & -2.158907 & -0.729637 & 6 & -2.530177 & 1.109452 & -2.447324 \\
\hline 6 & 3.400145 & 0.528712 & -1.195269 & 1 & -2.440626 & 0.131573 & -2.939007 \\
\hline 6 & 3.025575 & -0.629902 & 1.696063 & 1 & -3.014674 & 1.790733 & -3.161521 \\
\hline 6 & -2.688144 & -1.773131 & -0.707643 & 1 & -1.515461 & 1.487942 & -2.264838 \\
\hline 6 & -3.117952 & -0.161577 & 1.727743 & 6 & 2.559747 & 0.736119 & -2.461413 \\
\hline 6 & -3.359227 & 1.042400 & -1.159031 & 1 & 2.286361 & -0.212743 & -2.942362 \\
\hline 6 & 0.959269 & -2.794592 & -0.686480 & 1 & 1.633014 & 1.287636 & -2.252839 \\
\hline 6 & 0.850564 & -4.182556 & -0.795688 & 1 & 3.135619 & 1.320144 & -3.193778 \\
\hline 6 & -0.399702 & -4.790753 & -0.829737 & 6 & 2.299260 & -1.788038 & 2.387101 \\
\hline 6 & -1.436978 & -2.610618 & -0.675099 & 1 & 2.489755 & -2.755793 & 1.908144 \\
\hline 6 & -1.542096 & -3.998920 & -0.783617 & 1 & 2.657342 & -1.859295 & 3.424040 \\
\hline 1 & 1.753425 & -4.792674 & -0.854789 & 1 & 1.213295 & -1.637696 & 2.418367 \\
\hline 1 & -0.483229 & -5.873624 & -0.901559 & 6 & -2.677409 & 1.048992 & 2.550230 \\
\hline 1 & -2.527930 & -4.464218 & -0.833786 & 1 & -3.105822 & 0.971444 & 3.560082 \\
\hline 1 & 2.639448 & -2.061358 & -1.778415 & 1 & -1.586186 & 1.095009 & 2.648349 \\
\hline 1 & 3.097372 & -2.772144 & -0.245603 & 1 & -3.016474 & 1.999519 & 2.122067 \\
\hline 1 & -3.543182 & -2.259663 & -0.216971 & 6 & -3.472425 & 2.443706 & -0.560787 \\
\hline 1 & -2.992219 & -1.626001 & -1.754006 & 1 & -3.873414 & 3.132799 & -1.318334 \\
\hline 6 & -4.642908 & -0.236605 & 1.719611 & 1 & -4.160821 & 2.467708 & 0.293361 \\
\hline 1 & -5.102473 & 0.702321 & 1.389635 & 1 & -2.503474 & 2.838440 & -0.231634 \\
\hline 1 & -5.024781 & -1.050842 & 1.090281 & 6 & 3.738225 & 1.889696 & -0.588690 \\
\hline 1 & -4.996858 & -0.425073 & 2.743563 & 1 & 4.187409 & 2.534099 & -1.358962 \\
\hline 6 & -4.757416 & 0.538473 & -1.518590 & 1 & 2.850652 & 2.402846 & -0.200974 \\
\hline 1 & -4.749415 & -0.469046 & -1.951063 & 1 & 4.467924 & 1.804065 & 0.226126 \\
\hline 1 & -5.441387 & 0.535840 & -0.664890 & 6 & 2.792480 & 0.636505 & 2.519956 \\
\hline 1 & -5.189554 & 1.208950 & -2.275994 & 1 & 3.270619 & 1.521499 & 2.083530 \\
\hline 6 & 4.694652 & -0.181048 & -1.591492 & 1 & 1.723609 & 0.853161 & 2.634606 \\
\hline 1 & 5.376853 & -0.324888 & -0.748464 & 1 & 3.218534 & 0.494114 & 3.523762 \\
\hline 1 & 4.518957 & -1.157971 & -2.057126 & 77 & -0.018536 & 0.104342 & -0.0746 \\
\hline 1 & 5.222865 & 0.436597 & -2.332759 & 6 & 0.215913 & 3.249289 & -0.646333 \\
\hline 6 & 4.519612 & -0.943741 & 1.667537 & 1 & -0.641797 & 3.113926 & -1.326434 \\
\hline 1 & 4.760534 & -1.807524 & 1.034896 & 6 & 1.495169 & 5.124562 & 0.528673 \\
\hline 1 & 5.116939 & -0.088684 & 1.330149 & 1 & 1.645709 & 4.533454 & 1.440987 \\
\hline 1 & 4.853221 & -1.185629 & 2.687088 & 6 & 0.153546 & 2.228177 & 0.495085 \\
\hline 6 & -2.573774 & -1.422303 & 2.406582 & 1 & -0.033701 & -0.176426 & $1.4385=$ \\
\hline
\end{tabular}




$\begin{array}{rrrrrrrr}1 & 1.040471 & 2.377481 & 1.131603 & 1 & -0.647433 & 4.897764 & 0.442987 \\ 1 & -0.693921 & 2.524827 & 1.139044 & 1 & 1.473792 & 6.181063 & 0.822030 \\ 1 & 1.109384 & 3.081251 & -1.274029 & 1 & 2.378986 & 4.978833 & -0.108760 \\ 6 & 0.227055 & 4.715980 & -0.203208 & 1 & 0.097250 & 5.366084 & -1.082621\end{array}$

TS2

The smallest/imaginary frequencies is: $-733.8879 \mathrm{~cm}(-1)$

Zero-point correction=

Thermal correction to Energy=

Thermal correction to Enthalpy=

Thermal correction to Gibbs Free Energy=

Sum of electronic and zero-point Energies=

Sum of electronic and thermal Energies=

Sum of electronic and thermal Enthalpies=

Sum of electronic and thermal Free Energies=
0.742585

0.787837

0.788860

0.669283

$-1884.375422$

$-1884.330170$

$-1884.329147$

$-1884.448724$

Cartesian Coordinates

$\begin{array}{crrr}6 & -0.016822 & 1.679032 & -0.578335 \\ 15 & -2.321951 & 0.153638 & 0.249827 \\ 15 & 2.347103 & 0.136500 & 0.045555 \\ 6 & -2.290633 & 1.993401 & 0.401031 \\ 6 & -3.288406 & -0.110870 & -1.395166 \\ 6 & -3.397407 & -0.397354 & 1.724743 \\ 6 & 2.391850 & 1.488850 & -1.223449 \\ 6 & 3.116972 & 0.901962 & 1.614030 \\ 6 & 3.568370 & -1.106161 & -0.740145 \\ 6 & -1.149444 & 2.511057 & -0.426319 \\ 6 & -1.190003 & 3.804804 & -0.946697 \\ 6 & -0.078510 & 4.339475 & -1.590850 \\ 6 & 1.113885 & 2.272723 & -1.172563 \\ 6 & 1.079786 & 3.576241 & -1.675336 \\ 1 & -2.093359 & 4.404732 & -0.824014 \\ 1 & -0.107149 & 5.348495 & -1.997477 \\ 1 & 1.975942 & 3.995643 & -2.135587 \\ 1 & -3.248317 & 2.472066 & 0.167535 \\ 1 & -2.078333 & 2.198120 & 1.460576 \\ 1 & 3.277390 & 2.130490 & -1.108715 \\ 1 & 2.492984 & 0.984935 & -2.195637 \\ 6 & 4.622553 & 1.145947 & 1.532484 \\ 1 & 5.199761 & 0.215095 & 1.552924 \\ 1 & 4.915944 & 1.723059 & 0.646667 \\ 1 & 4.929904 & 1.728556 & 2.412946 \\ 6 & 4.920312 & -0.523499 & -1.166209 \\ 1 & 4.818170 & 0.389351 & -1.765236 \\ 1 & 5.589433 & -0.313026 & -0.329524 \\ 1 & 5.425377 & -1.266354 & -1.801320 \\ 6 & -4.343998 & 0.975508 & -1.622375 \\ 1 & -5.049497 & 1.085813 & -0.792524 \\ 1 & -3.886630 & 1.952316 & -1.820240\end{array}$

$\begin{array}{lrrr}1 & -4.929896 & 0.710792 & -2.514341 \\ 6 & -4.789520 & 0.232518 & 1.724193 \\ 1 & -4.755858 & 1.328475 & 1.681216 \\ 1 & -5.413293 & -0.128127 & 0.897941 \\ 1 & -5.305104 & -0.038677 & 2.657231 \\ 6 & 2.444954 & 2.255912 & 1.861697 \\ 1 & 2.690350 & 2.991782 & 1.085937 \\ 1 & 1.354647 & 2.175020 & 1.924964 \\ 1 & 2.809920 & 2.654061 & 2.819489 \\ 6 & 2.901827 & -1.616482 & -2.026175 \\ 1 & 3.066478 & -0.926736 & -2.862744 \\ 1 & 3.345222 & -2.580527 & -2.315047 \\ 1 & 1.821935 & -1.747161 & -1.924177 \\ 6 & -2.307460 & -0.034635 & -2.568448 \\ 1 & -1.729622 & 0.896175 & -2.572729 \\ 1 & -1.594799 & -0.865799 & -2.566155 \\ 1 & -2.884889 & -0.080649 & -3.503668 \\ 6 & -2.667656 & 0.038614 & 2.999509 \\ 1 & -2.622362 & 1.128113 & 3.111256 \\ 1 & -3.215905 & -0.351567 & 3.868662 \\ 1 & -1.641609 & -0.345219 & 3.038822 \\ 6 & 2.830916 & -0.010906 & 2.807174 \\ 1 & 3.238623 & 0.449947 & 3.718748 \\ 1 & 1.754790 & -0.160499 & 2.950359 \\ 1 & 3.300241 & -0.997040 & 2.701942 \\ 6 & 3.820840 & -2.264590 & 0.223334 \\ 1 & 4.420405 & -3.038265 & -0.278177 \\ 1 & 4.385002 & -1.942319 & 1.107352 \\ 1 & 2.894592 & -2.736174 & 0.572039 \\ 6 & -3.972493 & -1.476665 & -1.450329 \\ 1 & -4.346807 & -1.641281 & -2.471070 \\ 1 & -3.286699 & -2.303121 & -1.222608 \\ & & & \end{array}$




$\begin{array}{crrrrrrr}1 & -4.836174 & -1.544782 & -0.779015 & 1 & -0.664051 & -2.299595 & 2.049387 \\ 6 & -3.542930 & -1.920577 & 1.781436 & 1 & 1.054438 & -2.597979 & 1.545503 \\ 1 & -4.151116 & -2.325642 & 0.968665 & 1 & -1.436699 & -2.653581 & -0.228708 \\ 1 & -2.575445 & -2.433929 & 1.771414 & 6 & 0.365522 & -3.621418 & -0.906756 \\ 1 & -4.043464 & -2.190895 & 2.722349 & 1 & 1.452190 & -3.500930 & -0.818414 \\ 77 & 0.012562 & -0.278828 & 0.250451 & 1 & 0.133992 & -4.577067 & -0.410630 \\ 6 & -0.352641 & -2.564507 & -0.094547 & 6 & -0.056492 & -3.701042 & -2.361760 \\ 1 & -0.127293 & -1.387187 & -1.112787 & 1 & 0.443500 & -4.527951 & -2.878708 \\ 6 & 0.065110 & -2.248025 & 1.242594 & 1 & -1.139575 & -3.864160 & -2.448013 \\ 1 & 0.058936 & 0.582825 & 1.634611 & 1 & 0.178315 & -2.777776 & -2.907296\end{array}$

TS1'

The smallest/imaginary frequencies is: $-530.7176 \mathrm{~cm}(-1)$

Zero-point correction $=$

Thermal correction to Energy=

Thermal correction to Enthalpy=

Thermal correction to Gibbs Free Energy=

Sum of electronic and zero-point Energies=

Sum of electronic and thermal Energies=

Sum of electronic and thermal Enthalpies=

Sum of electronic and thermal Free Energies=
0.744986

0.791230

0.792253

0.669387

$-1884.382465$

$-1884.336222$

$-1884.335198$

$-1884.458065$

Cartesian Coordinates

$\begin{array}{cccc}6 & -0.051546 & 1.702180 & -0.709986 \\ 15 & -2.332724 & 0.161567 & 0.073757 \\ 15 & 2.292273 & 0.186955 & 0.205703 \\ 6 & -2.518131 & 1.528395 & -1.167151 \\ 6 & -3.772320 & -0.991987 & -0.436964 \\ 6 & -2.765526 & 0.954397 & 1.760108 \\ 6 & 2.282259 & 2.044778 & 0.106210 \\ 6 & 3.112027 & -0.080536 & 1.914216 \\ 6 & 3.534454 & -0.267581 & -1.172901 \\ 6 & -1.222101 & 2.284667 & -1.247783 \\ 6 & -1.215673 & 3.569886 & -1.796223 \\ 6 & -0.048515 & 4.324072 & -1.809929 \\ 6 & 1.094291 & 2.527155 & -0.667487 \\ 6 & 1.103119 & 3.805945 & -1.226542 \\ 1 & -2.136720 & 3.983468 & -2.209465 \\ 1 & -0.042422 & 5.320487 & -2.248082 \\ 1 & 2.011926 & 4.407887 & -1.184171 \\ 1 & -2.748642 & 1.040184 & -2.125695 \\ 1 & -3.365987 & 2.193335 & -0.944991 \\ 1 & 2.176183 & 2.396427 & 1.142981 \\ 1 & 3.231921 & 2.458623 & -0.259376 \\ 6 & 4.205570 & 0.939809 & 2.244556 \\ 1 & 5.030832 & 0.938322 & 1.526009 \\ 1 & 3.823258 & 1.963724 & 2.318663 \\ 1 & 4.631469 & 0.688312 & 3.226917 \\ 6 & 4.927306 & 0.338912 & -1.014138\end{array}$

$\begin{array}{lrrr}1 & 4.915708 & 1.426263 & -0.872948 \\ 1 & 5.484948 & -0.113064 & -0.185444 \\ 1 & 5.504500 & 0.141995 & -1.929772 \\ 6 & -5.047922 & -0.245429 & -0.839042 \\ 1 & -5.464249 & 0.379910 & -0.046089 \\ 1 & -4.897122 & 0.382969 & -1.724398 \\ 1 & -5.816112 & -0.987342 & -1.102843 \\ 6 & -4.225629 & 1.372958 & 1.920228 \\ 1 & -4.553455 & 2.063791 & 1.132954 \\ 1 & -4.915508 & 0.522730 & 1.949430 \\ 1 & -4.336020 & 1.903772 & 2.877106 \\ 6 & 2.001135 & 0.063040 & 2.956992 \\ 1 & 1.479806 & 1.026675 & 2.886755 \\ 1 & 1.247520 & -0.726614 & 2.854354 \\ 1 & 2.440154 & -0.005888 & 3.963489 \\ 6 & 2.909362 & 0.263060 & -2.466455 \\ 1 & 2.827108 & 1.356978 & -2.474960 \\ 1 & 3.540987 & -0.028208 & -3.318274 \\ 1 & 1.903947 & -0.145519 & -2.632348 \\ 6 & -3.307301 & -1.778184 & -1.663970 \\ 1 & -2.917262 & -1.134337 & -2.463301 \\ 1 & -2.534116 & -2.506264 & -1.408338 \\ 1 & -4.161224 & -2.333021 & -2.078813 \\ 6 & -1.907076 & 2.209203 & 1.942916 \\ 1 & -2.132501 & 2.988572 & 1.204320 \\ 1 & -2.111624 & 2.627137 & 2.939635\end{array}$




$\begin{array}{lrrr}1 & -0.837202 & 1.982953 & 1.875200 \\ 6 & 3.709888 & -1.479215 & 2.045728 \\ 1 & 3.974289 & -1.658300 & 3.098127 \\ 1 & 3.007102 & -2.265971 & 1.748290 \\ 1 & 4.630941 & -1.594166 & 1.462045 \\ 6 & 3.693727 & -1.780794 & -1.303956 \\ 1 & 4.212670 & -2.229171 & -0.450569 \\ 1 & 2.731484 & -2.287887 & -1.417637 \\ 1 & 4.290804 & -2.002603 & -2.200642 \\ 6 & -4.100715 & -1.996769 & 0.666525 \\ 1 & -4.779836 & -2.763542 & 0.265655 \\ 1 & -3.206370 & -2.514106 & 1.038292 \\ 1 & -4.608090 & -1.534680 & 1.521164 \\ 6 & -2.394673 & -0.028060 & 2.873049 \\ 1 & -2.989199 & -0.948036 & 2.846972 \\ 1 & -1.334862 & -0.304853 & 2.813625\end{array}$

$\begin{array}{cccc}1 & -2.570257 & 0.447592 & 3.849241 \\ 77 & -0.020535 & -0.287099 & -0.032800 \\ 6 & -0.155984 & -2.590556 & -0.013405 \\ 1 & -1.181810 & -2.860079 & 0.282564 \\ 6 & 0.790889 & -3.515759 & 0.746920 \\ 1 & 0.758084 & -3.352120 & 1.832290 \\ 1 & 0.545900 & -4.573421 & 0.575576 \\ 1 & 1.829568 & -3.369301 & 0.418754 \\ 1 & -0.004297 & -1.491548 & 1.054780 \\ 6 & -0.030229 & -2.834593 & -1.524440 \\ 1 & -0.622280 & -2.099199 & -2.083716 \\ 6 & -0.450067 & -4.243204 & -1.947157 \\ 1 & -1.447379 & -4.501204 & -1.563974 \\ 1 & -0.485380 & -4.327869 & -3.040146 \\ 1 & 0.245948 & -5.009314 & -1.585231 \\ 1 & 1.007127 & -2.670443 & -1.839747\end{array}$

\section{$5^{\prime}$}

The smallest/imaginary frequencies is: $19.9696 \mathrm{~cm}(-1)$
Zero-point correction=
Thermal correction to Energy=
Thermal correction to Enthalpy=
0.744114
0.791439
0.792462
Thermal correction to Gibbs Free Energy $=\quad 0.666810$
Sum of electronic and zero-point Energies $=\quad-1884.394163$
Sum of electronic and thermal Energies $=\quad-1884.346839$
Sum of electronic and thermal Enthalpies $=\quad-1884.345816$
Sum of electronic and thermal Free Energies $=\quad-1884.471468$

Cartesian Coordinates

$\begin{array}{cccc}6 & -0.056314 & 1.870239 & -0.593434 \\ 15 & -2.335187 & 0.105163 & -0.055825 \\ 15 & 2.329385 & 0.266166 & 0.003103 \\ 6 & -2.534494 & 1.735762 & -0.925717 \\ 6 & -3.420447 & -1.014628 & -1.148384 \\ 6 & -3.163920 & 0.449897 & 1.621253 \\ 6 & 2.458194 & 2.023486 & -0.585553 \\ 6 & 3.115953 & 0.331601 & 1.729019 \\ 6 & 3.442710 & -0.638547 & -1.248982 \\ 6 & -1.279651 & 2.549172 & -0.806049 \\ 6 & -1.347341 & 3.936611 & -0.941242 \\ 6 & -0.188530 & 4.704411 & -0.899831 \\ 6 & 1.105285 & 2.677939 & -0.624229 \\ 6 & 1.038127 & 4.067594 & -0.756283 \\ 1 & -2.316005 & 4.419296 & -1.080897 \\ 1 & -0.240524 & 5.787721 & -0.991655 \\ 1 & 1.958457 & 4.653995 & -0.752830 \\ 1 & -2.706577 & 1.500659 & -1.985741 \\ 1 & -3.429731 & 2.276970 & -0.588785 \\ 1 & 3.191264 & 2.592071 & 0.003300\end{array}$

$\begin{array}{lrrr}1 & 2.862125 & 1.998490 & -1.606652 \\ 6 & 4.611428 & 0.639342 & 1.705197 \\ 1 & 5.199447 & -0.198596 & 1.312877 \\ 1 & 4.855496 & 1.537006 & 1.123072 \\ 1 & 4.954701 & 0.817457 & 2.734599 \\ 6 & 4.766939 & 0.059345 & -1.567285 \\ 1 & 4.644254 & 1.094671 & -1.904279 \\ 1 & 5.459746 & 0.058093 & -0.720787 \\ 1 & 5.258911 & -0.485645 & -2.386125 \\ 6 & -4.739493 & -0.396186 & -1.616081 \\ 1 & -5.446008 & -0.224316 & -0.799379 \\ 1 & -4.602987 & 0.549068 & -2.153451 \\ 1 & -5.221200 & -1.094214 & -2.316639 \\ 6 & -4.668871 & 0.688794 & 1.520124 \\ 1 & -4.934617 & 1.467430 & 0.794512 \\ 1 & -5.216506 & -0.226151 & 1.265724 \\ 1 & -5.039821 & 1.022259 & 2.500174 \\ 6 & 2.399680 & 1.443092 & 2.503226 \\ 1 & 2.584421 & 2.440575 & 2.087289 \\ 1 & 1.314083 & 1.290061 & 2.536981\end{array}$




$\begin{array}{lrrr}1 & 2.769848 & 1.446399 & 3.538269 \\ 6 & 2.605192 & -0.694061 & -2.532126 \\ 1 & 2.365730 & 0.303393 & -2.923828 \\ 1 & 3.165857 & -1.227137 & -3.313345 \\ 1 & 1.658380 & -1.230278 & -2.380073 \\ 6 & -2.564874 & -1.289546 & -2.390634 \\ 1 & -2.350739 & -0.374902 & -2.959817 \\ 1 & -1.608010 & -1.760276 & -2.136181 \\ 1 & -3.103711 & -1.971928 & -3.063799 \\ 6 & -2.508203 & 1.708520 & 2.197463 \\ 1 & -2.720820 & 2.607757 & 1.607531 \\ 1 & -2.903399 & 1.877566 & 3.209463 \\ 1 & -1.418651 & 1.609116 & 2.274393 \\ 6 & 2.890176 & -0.984037 & 2.472243 \\ 1 & 3.320286 & -0.902274 & 3.481028 \\ 1 & 1.823516 & -1.211042 & 2.577900 \\ 1 & 3.370473 & -1.837772 & 1.980189 \\ 6 & 3.750965 & -2.064430 & -0.798266 \\ 1 & 4.286669 & -2.588184 & -1.603300 \\ 1 & 4.399164 & -2.086413 & 0.086839 \\ 1 & 2.844985 & -2.638084 & -0.575588 \\ 6 & -3.723418 & -2.332798 & -0.441517\end{array}$

1

$$
\begin{array}{ccc}
-4.187554 & -3.031818 & -1.152521 \\
-2.820125 & -2.813296 & -0.048751 \\
-4.429919 & -2.199456 & 0.387725 \\
-2.915203 & -0.698325 & 2.594451 \\
-3.337110 & -1.648691 & 2.247228 \\
-1.845747 & -0.849127 & 2.774034 \\
-3.389173 & -0.460226 & 3.557831 \\
0.021966 & -0.207622 & -0.050828 \\
0.083950 & -3.274572 & -0.731721 \\
0.540983 & -2.831677 & -1.634640 \\
0.301740 & -2.356097 & 0.478106 \\
-0.014613 & -0.021065 & 1.478700 \\
1.371287 & -2.454131 & 0.727684 \\
-0.992339 & -3.348633 & -0.958117 \\
0.633924 & -4.692808 & -0.581035 \\
1.698421 & -4.680304 & -0.306306 \\
0.539788 & -5.266572 & -1.512272 \\
-0.426272 & -2.917513 & 1.696235 \\
-1.516225 & -2.952454 & 1.552644 \\
-0.111009 & -3.944506 & 1.950940 \\
-0.249308 & -2.312618 & 2.596503 \\
0.106382 & -5.254844 & 0.199237
\end{array}
$$

TS2'

The smallest/imaginary frequencies is: $-750.6268 \mathrm{~cm}(-1)$

$\begin{array}{lc}\text { Zero-point correction }= & 0.742802 \\ \text { Thermal correction to Energy= } & 0.788105 \\ \text { Thermal correction to Enthalpy= } & 0.789128 \\ \text { Thermal correction to Gibbs Free Energy= } & 0.669149 \\ \text { Sum of electronic and zero-point Energies }= & -1884.372436 \\ \text { Sum of electronic and thermal Energies= } & -1884.327134 \\ \text { Sum of electronic and thermal Enthalpies }= & -1884.326110 \\ \text { Sum of electronic and thermal Free Energies }= & -1884.446089\end{array}$

Zero-point correction=

Thermal correction to Gibbs Free Energy=

Sum of electronic and zero-point Energies=

Sum of electronic and thermal Enthalpies=

Cartesian Coordinates

$\begin{array}{rrrr}1 & -2.223114 & 4.205888 & 1.173880 \\ 1 & -0.395795 & 5.646563 & 0.287280 \\ 1 & 1.642110 & 4.586296 & -0.665984 \\ 1 & -3.274963 & 1.975074 & 1.309205 \\ 1 & -2.001326 & 1.135914 & 2.206865 \\ 1 & 3.061521 & 2.538759 & -0.690510 \\ 1 & 2.130158 & 1.743938 & -1.968283 \\ 6 & 4.416974 & 1.135641 & 1.635916 \\ 1 & 5.166212 & 0.423641 & 1.274878 \\ 1 & 4.498731 & 2.059526 & 1.049526 \\ 1 & 4.694003 & 1.389619 & 2.669120 \\ 6 & 4.755180 & 0.481539 & -1.599121 \\ 1 & 4.352886 & 1.250424 & -2.268150 \\ 1 & 5.247070 & 0.982026 & -0.761568\end{array}$




$\begin{array}{lrrr}1 & 5.535645 & -0.052568 & -2.160601 \\ 6 & -4.397268 & 1.634666 & -0.994661 \\ 1 & -5.080106 & 1.341512 & -0.189848 \\ 1 & -3.901689 & 2.568683 & -0.704579 \\ 1 & -5.013140 & 1.858222 & -1.877751 \\ 6 & -4.707565 & -0.634476 & 1.694758 \\ 1 & -4.549908 & 0.283852 & 2.273023 \\ 1 & -5.399844 & -0.413119 & 0.873987 \\ 1 & -5.215466 & -1.351193 & 2.356645 \\ 6 & 2.113736 & 1.592931 & 2.368810 \\ 1 & 2.142886 & 2.585549 & 1.904682 \\ 1 & 1.068485 & 1.271224 & 2.424002 \\ 1 & 2.491417 & 1.695513 & 3.396572 \\ 6 & 3.044575 & -1.057786 & -2.463672 \\ 1 & 2.413963 & -0.308557 & -2.959520 \\ 1 & 3.838658 & -1.339346 & -3.170136 \\ 1 & 2.438984 & -1.950132 & -2.284085 \\ 6 & -2.441430 & 1.146121 & -2.412670 \\ 1 & -1.828289 & 1.959112 & -2.008380 \\ 1 & -1.765683 & 0.397055 & -2.838546 \\ 1 & -3.049639 & 1.555636 & -3.232566 \\ 6 & -2.545144 & -1.620634 & 2.422002 \\ 1 & -2.288527 & -0.751158 & 3.038241 \\ 1 & -3.117998 & -2.311621 & 3.056720 \\ 1 & -1.607565 & -2.109653 & 2.134616 \\ 6 & 2.965106 & -0.719400 & 2.460030 \\ 1 & 3.353735 & -0.513664 & 3.467961 \\ 1 & 1.938275 & -1.088276 & 2.565312\end{array}$

1

The smallest/imaginary frequencies is: 30.3453 $\mathrm{cm}(-1)$

Zero-point correction=

0.743726

(Hartree/Particle)

Thermal correction to Energy=

0.789395

Thermal correction to Enthalpy=

0.790418

Thermal correction to Gibbs Free Energy= 0.669855

Sum of electronic and zero-point Energies= 1884.394441

Sum of electronic and thermal Energies= 1884.348772

Sum of electronic and thermal Enthalpies= 1884.347749

Sum of electronic and thermal Free Energies= 1884.468312

\begin{tabular}{|c|c|c|c|}
\hline 1 & 3.577381 & -1.520100 & 2.030728 \\
\hline 6 & 4.377566 & -1.705654 & -0.474847 \\
\hline 1 & 4.994462 & -2.247228 & -1.206738 \\
\hline 1 & 5.047920 & -1.377773 & 0.327118 \\
\hline 1 & 3.669659 & -2.426223 & -0.048392 \\
\hline 6 & -4.141428 & -0.605937 & -2.034825 \\
\hline 1 & -4.520402 & -0.262109 & -3.007970 \\
\hline 1 & -3.507570 & -1.481552 & -2.224481 \\
\hline 1 & -5.010336 & -0.928934 & -1.451090 \\
\hline 6 & -3.735685 & -2.558619 & 0.467696 \\
\hline 1 & -4.557444 & -2.443072 & -0.243307 \\
\hline 1 & -2.883144 & -2.984710 & -0.071644 \\
\hline 1 & -4.056287 & -3.305836 & 1.207449 \\
\hline 77 & -0.026307 & -0.376093 & -0.140161 \\
\hline 6 & -0.283375 & -1.926535 & -1.783313 \\
\hline 1 & -0.047796 & -0.409502 & -1.870615 \\
\hline 6 & -0.113489 & -2.572830 & -0.504350 \\
\hline 1 & 0.031887 & -0.401671 & 1.498361 \\
\hline 1 & -1.030655 & -2.952537 & -0.049733 \\
\hline 1 & -1.280658 & -1.907029 & -2.229573 \\
\hline 1 & 0.481916 & -2.104843 & -2.541886 \\
\hline 6 & 1.031939 & -3.531642 & -0.285540 \\
\hline 1 & 1.958060 & -3.116045 & -0.702508 \\
\hline 1 & 0.845647 & -4.462410 & -0.851834 \\
\hline 6 & 1.238198 & -3.891638 & 1.174412 \\
\hline 1 & 0.337502 & -4.363609 & 1.590922 \\
\hline 1 & 2.069741 & -4.594564 & 1.312093 \\
\hline 1 & 1.439929 & -2.994341 & 1.771607 \\
\hline 6 & 0.008310 & 1.737927 & -0.396829 \\
\hline 15 & -2.328531 & 0.126780 & 0.209769 \\
\hline 15 & 2.359474 & 0.106289 & 0.008723 \\
\hline 6 & -2.291118 & 1.943224 & 0.546534 \\
\hline 6 & -3.321911 & 0.007525 & -1.429657 \\
\hline 6 & -3.398447 & -0.543186 & 1.652526 \\
\hline 6 & 2.397057 & 1.574192 & -1.119008 \\
\hline 6 & 3.126864 & 0.732943 & 1.642405 \\
\hline 6 & 3.591420 & -1.055732 & -0.876763 \\
\hline 6 & -1.133265 & 2.540292 & -0.195397 \\
\hline 6 & -1.173655 & 3.871643 & -0.611982 \\
\hline 6 & -0.060345 & 4.457098 & -1.205348 \\
\hline 6 & 1.132708 & 2.364875 & -0.961742 \\
\hline 6 & 1.096861 & 3.704008 & -1.359246 \\
\hline 1 & -2.081268 & 4.455725 & -0.453609 \\
\hline 1 & -0.089819 & 5.495607 & -1.529059 \\
\hline 1 & 1.988503 & 4.156115 & -1.795941 \\
\hline 1 & -3.242599 & 2.447495 & 0.341482 \\
\hline 1 & -2.099567 & 2.037815 & 1.624524 \\
\hline 1 & 3.294280 & 2.190594 & -0.965988 \\
\hline 1 & 2.462534 & 1.165500 & -2.138215 \\
\hline 6 & 4.628765 & 1.002256 & 1.573425 \\
\hline 1 & 5.218039 & 0.082456 & 1.490625 \\
\hline 1 & 4.905535 & 1.674287 & 0.75163 \\
\hline
\end{tabular}




$\begin{array}{lrrr}1 & 4.936960 & 1.493624 & 2.507537 \\ 6 & 4.906966 & -0.392590 & -1.300710 \\ 1 & 4.750698 & 0.528471 & -1.874416 \\ 1 & 5.575223 & -0.168795 & -0.466976 \\ 1 & 5.441746 & -1.090904 & -1.961047 \\ 6 & -4.444465 & 1.045588 & -1.524706 \\ 1 & -5.152129 & 1.021139 & -0.691413 \\ 1 & -4.045940 & 2.064052 & -1.610402 \\ 1 & -5.017929 & 0.851818 & -2.442515 \\ 6 & -4.828436 & -0.001511 & 1.636167 \\ 1 & -4.865491 & 1.093893 & 1.588679 \\ 1 & -5.426533 & -0.408207 & 0.812877 \\ 1 & -5.326566 & -0.299843 & 2.570303 \\ 6 & 2.440276 & 2.049279 & 2.018985 \\ 1 & 2.674014 & 2.859558 & 1.317726 \\ 1 & 1.351498 & 1.946547 & 2.078157 \\ 1 & 2.805243 & 2.357624 & 3.009293 \\ 6 & 2.918017 & -1.530345 & -2.169171 \\ 1 & 2.897962 & -0.740284 & -2.929882 \\ 1 & 3.496120 & -2.366718 & -2.587720 \\ 1 & 1.888766 & -1.858105 & -2.019336 \\ 6 & -2.377022 & 0.275592 & -2.603728 \\ 1 & -1.859771 & 1.238561 & -2.514917 \\ 1 & -1.614544 & -0.502409 & -2.702153 \\ 1 & -2.974243 & 0.297110 & -3.527032 \\ 6 & -2.751110 & -0.106315 & 2.971665 \\ 1 & -2.883744 & 0.963366 & 3.170526 \\ 1 & -3.239844 & -0.646408 & 3.794821 \\ 1 & -1.677572 & -0.324901 & 3.006521 \\ 6 & 2.859300 & -0.280754 & 2.755566\end{array}$

$\begin{array}{lrrr}1 & 3.274215 & 0.105198 & 3.697880 \\ 1 & 1.784240 & -0.439636 & 2.897878 \\ 1 & 3.332530 & -1.252220 & 2.566184 \\ 6 & 3.912416 & -2.255965 & 0.012998 \\ 1 & 4.509606 & -2.984220 & -0.554499 \\ 1 & 4.503775 & -1.969264 & 0.891134 \\ 1 & 3.013305 & -2.774171 & 0.367607 \\ 6 & -3.904464 & -1.393154 & -1.613427 \\ 1 & -4.298355 & -1.483800 & -2.635899 \\ 1 & -3.142920 & -2.174963 & -1.490406 \\ 1 & -4.735620 & -1.602142 & -0.930016 \\ 6 & -3.457488 & -2.073253 & 1.661834 \\ 1 & -3.857401 & -2.501727 & 0.738207 \\ 1 & -2.477856 & -2.522792 & 1.852478 \\ 1 & -4.121415 & -2.390907 & 2.478673 \\ 77 & 0.014716 & -0.304499 & 0.147065 \\ 6 & -0.373847 & -2.556618 & 0.100894 \\ 1 & 0.072679 & -0.565746 & -1.501466 \\ 6 & 0.145271 & -2.180441 & 1.348228 \\ 1 & -0.000893 & 0.315107 & 1.706493 \\ 1 & -0.493488 & -2.082202 & 2.224201 \\ 1 & 1.175643 & -2.449476 & 1.587326 \\ 1 & -1.459840 & -2.649696 & 0.023685 \\ 6 & 0.324979 & -3.506876 & -0.834163 \\ 1 & 1.410132 & -3.349241 & -0.810653 \\ 6 & -0.194965 & -3.484995 & -2.260200 \\ 1 & -0.058633 & -2.498648 & -2.721061 \\ 1 & 0.311537 & -4.230286 & -2.884993 \\ 1 & -1.270073 & -3.711117 & -2.286128 \\ 1 & 0.169974 & -4.520658 & -0.426988\end{array}$

alkene

The smallest/imaginary frequencies is: $100.3156 \mathrm{~cm}(-1)$

Zero-point correction=

Thermal correction to Energy=

Thermal correction to Enthalpy=

Thermal correction to Gibbs Free Energy=

Sum of electronic and zero-point Energies=

Sum of electronic and thermal Energies=

Sum of electronic and thermal Enthalpies=

Sum of electronic and thermal Free Energies=
0.107760

0.113806

0.114829

0.077345

$-156.981036$

$-156.974990$

$-156.973967$

$-157.011451$

Cartesian Coordinates 
$6 \quad-1.710601 \quad-0.247153 \quad-0.291107$

$\begin{array}{llll}1 & -0.798066 & 0.843797 & 1.322816\end{array}$

$-1.500725-0.545843-1.325023$

$\begin{array}{lllll}6 & 0.710861 & -0.295608 & 0.339250\end{array}$

$\begin{array}{lllll}1 & -1.910691 & -1.162100 & 0.280858\end{array}$

$\begin{array}{lllll}1 & -2.628496 & 0.351246 & -0.291288\end{array}$

$\begin{array}{lllll}6 & -0.537978 & 0.525457 & 0.301742\end{array}$

$\begin{array}{lllll}1 & -0.356020 & 1.443653 & -0.273922\end{array}$

$\begin{array}{lllll}1 & 0.648548 & -1.229648 & 0.905516\end{array}$

$\begin{array}{lllll}6 & 1.849405 & 0.015718 & -0.273785\end{array}$

$\begin{array}{lllll}1 & 1.948864 & 0.934303 & -0.852768\end{array}$

$\begin{array}{lllll}1 & 2.726462 & -0.625891 & -0.222788\end{array}$ 
7. NMR spectra of unreported amines and reaction mixtures

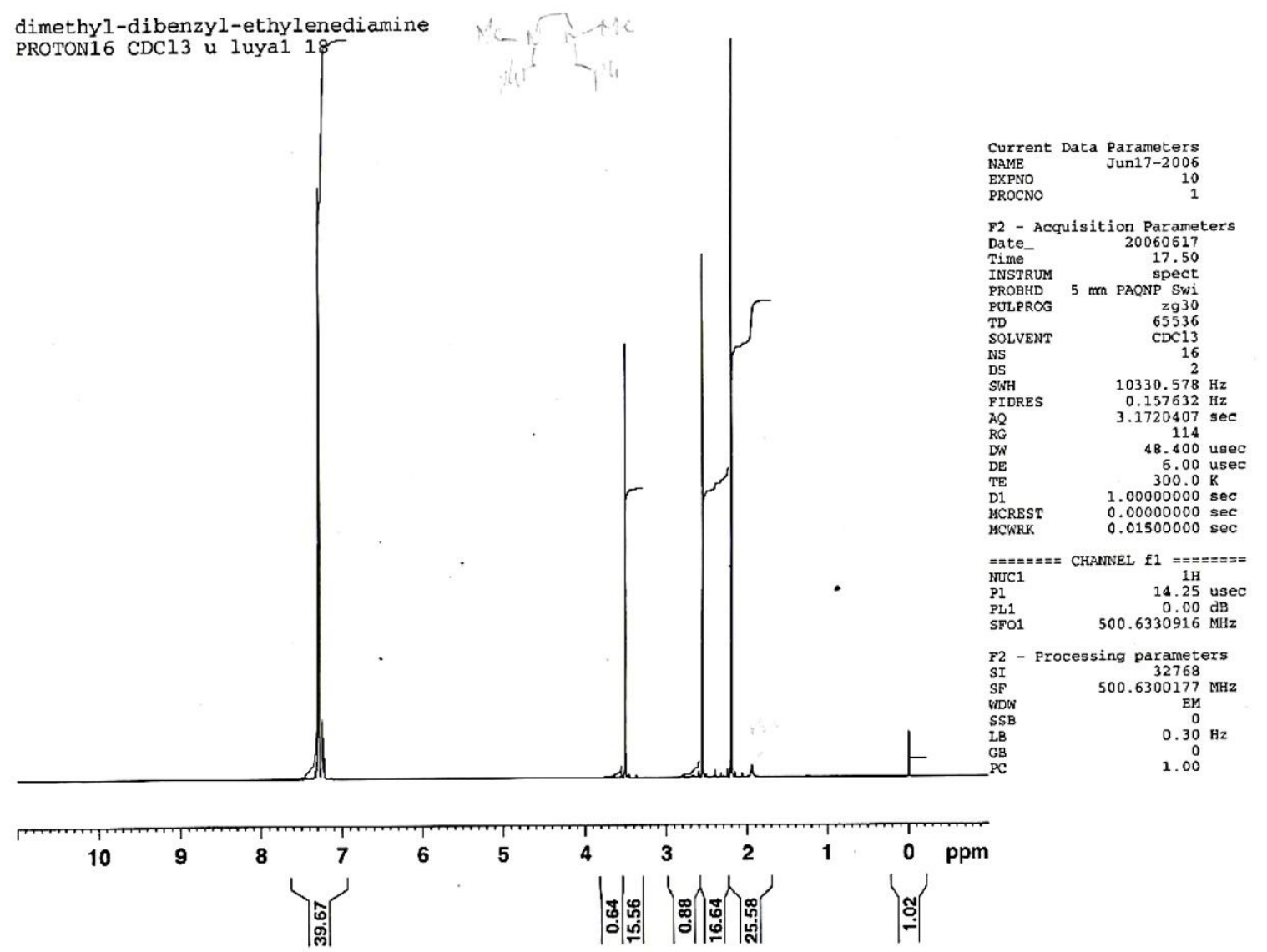

Figure S1. ${ }^{1} \mathrm{H}$ NMR spectrum of $N, N^{\prime}$-Dimethyl- $N, N^{\prime}$-dibenzylethylenediamine 


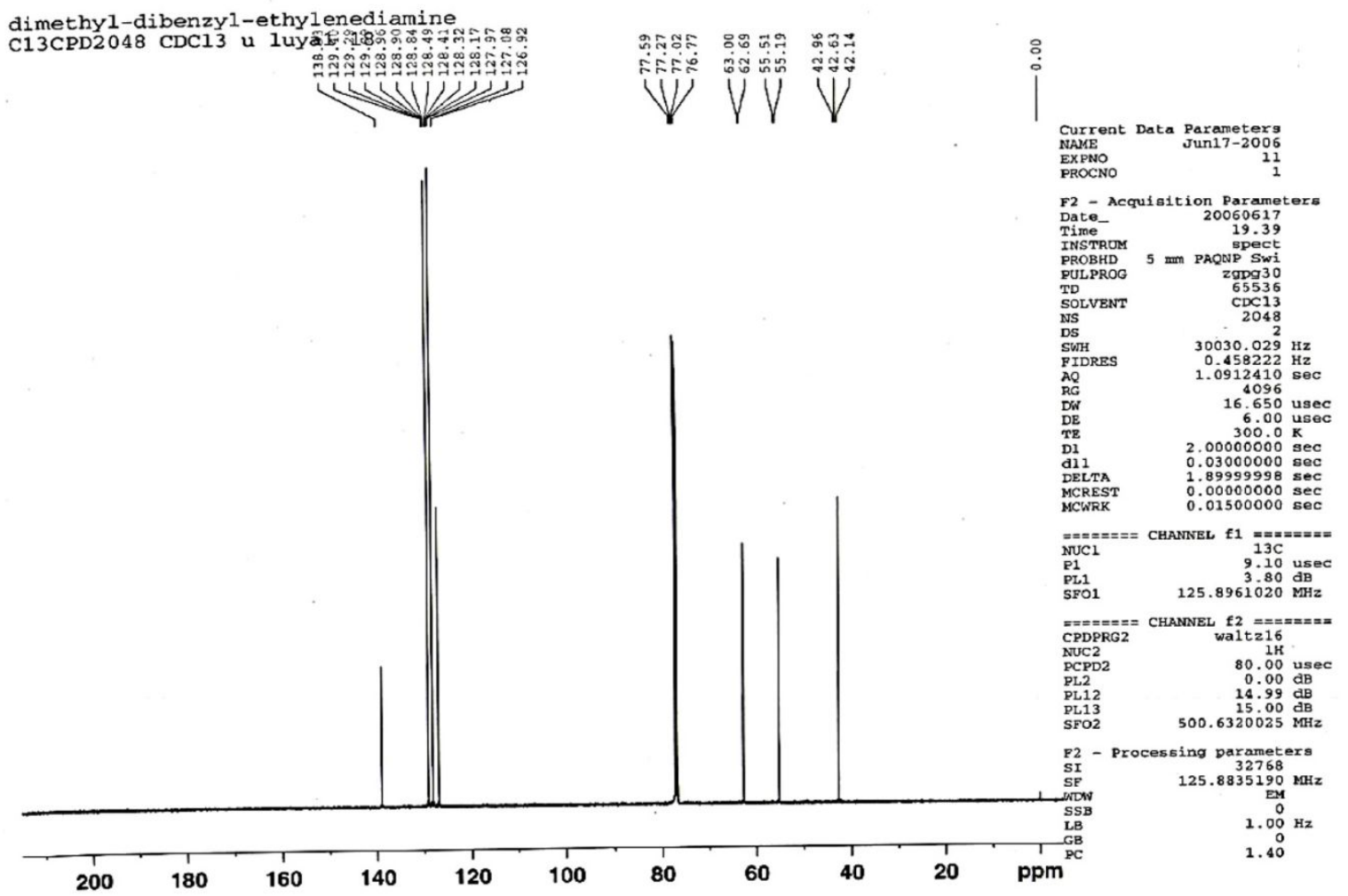

Figure S2. ${ }^{13} \mathrm{C}$ NMR spectrum of $N, N^{\prime}$-Dimethyl- $N, N^{\prime}$-dibenzylethylenediamine 
Table S10. Dehydrogenation Reactions Catalyzed by (iPr4PCP)Ir (2) with NBE as Hydrogen Acceptor a



(a) All reactions were run in $p$-xylene- $d_{10}$ and NBE was used as hydrogen acceptor. All yields were determined by ${ }^{1} \mathrm{H}$ NMR spectroscopy. A: $0.05 \mathrm{mmol}$ substrate $(0.1 \mathrm{M}), 2.3$ eq $(0.115 \mathrm{mmol}) \mathrm{NBE}, 15 \mathrm{~mol} \%$ $(4.0 \mathrm{mg}) 2,143^{\circ} \mathrm{C}, 45 \mathrm{~h}$, unless noted otherwise. B: $0.05 \mathrm{mmol}$ substrate $(0.1 \mathrm{M}), 2.0$ eq $(0.10 \mathrm{mmol}) \mathrm{NBE}$, $25 \mathrm{~mol} \%(6.6 \mathrm{mg}) 2,143{ }^{\circ} \mathrm{C}, 24 \mathrm{~h}$. 


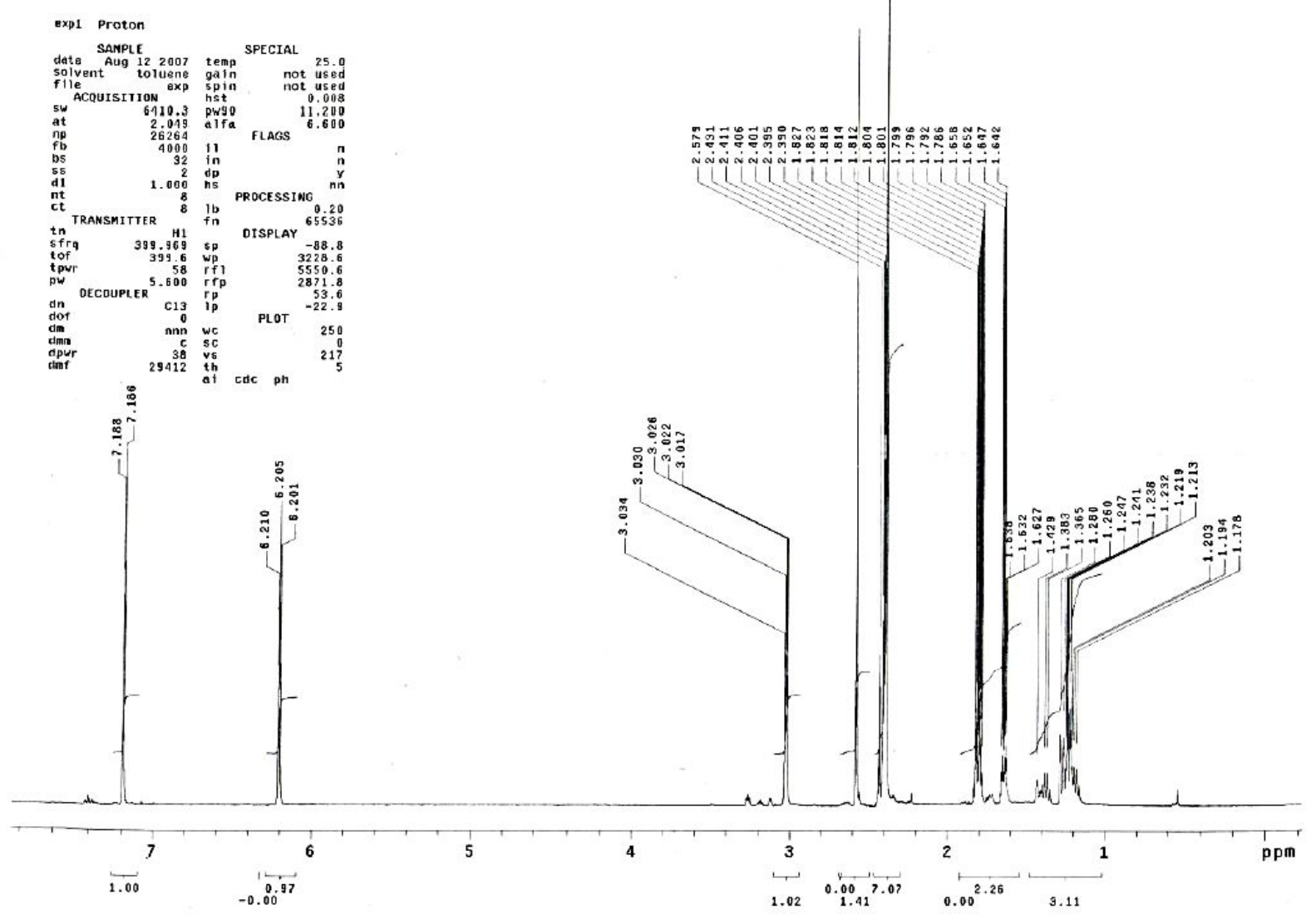

Figure S3. ${ }^{1} \mathrm{H}$ NMR spectrum at $t_{0}$ of reaction mixture of Table S10, Entry 1 

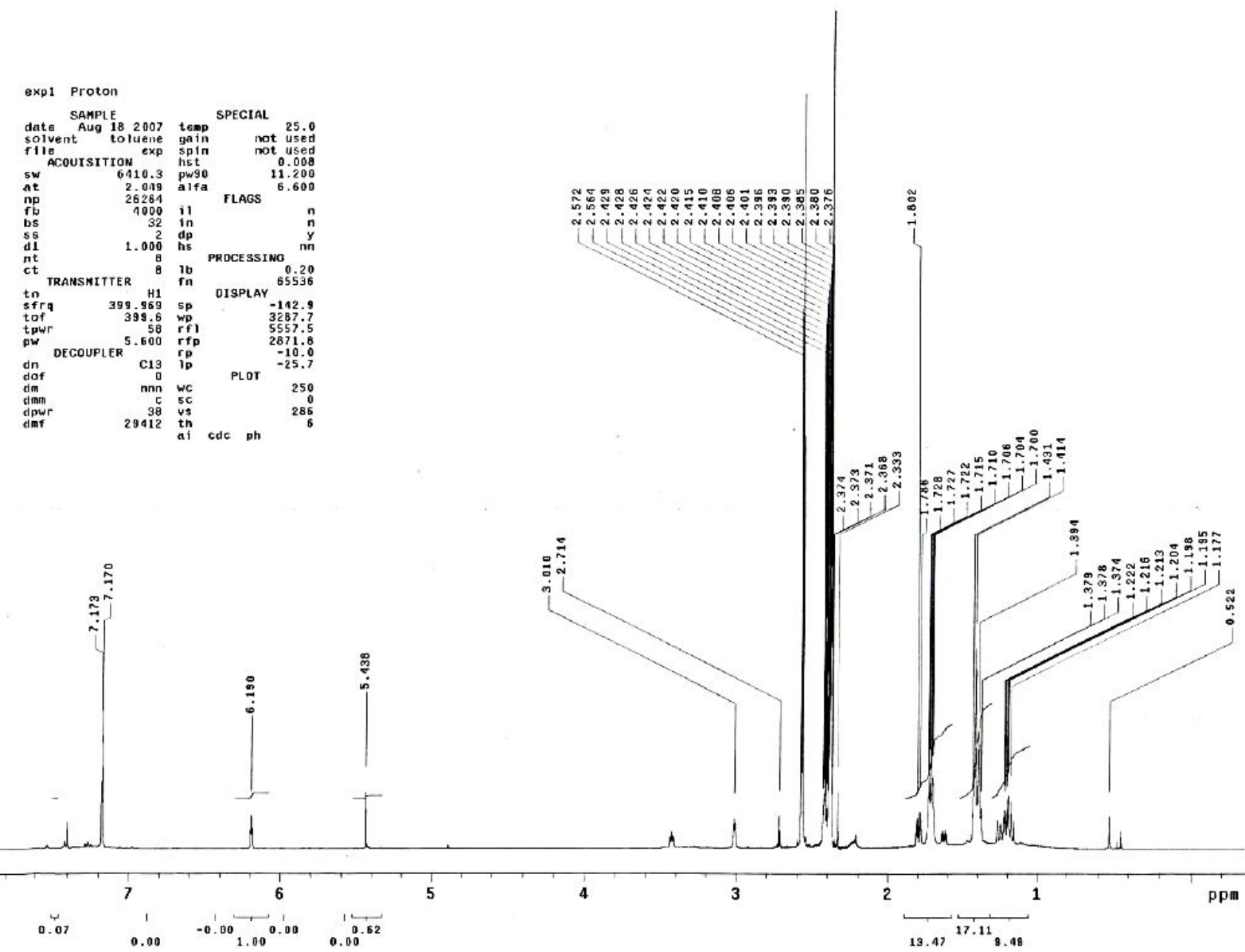

Figure S4. ${ }^{1} \mathrm{H}$ NMR spectrum of reaction mixture of Table S10 Entry $1 \mathbf{A}$ 

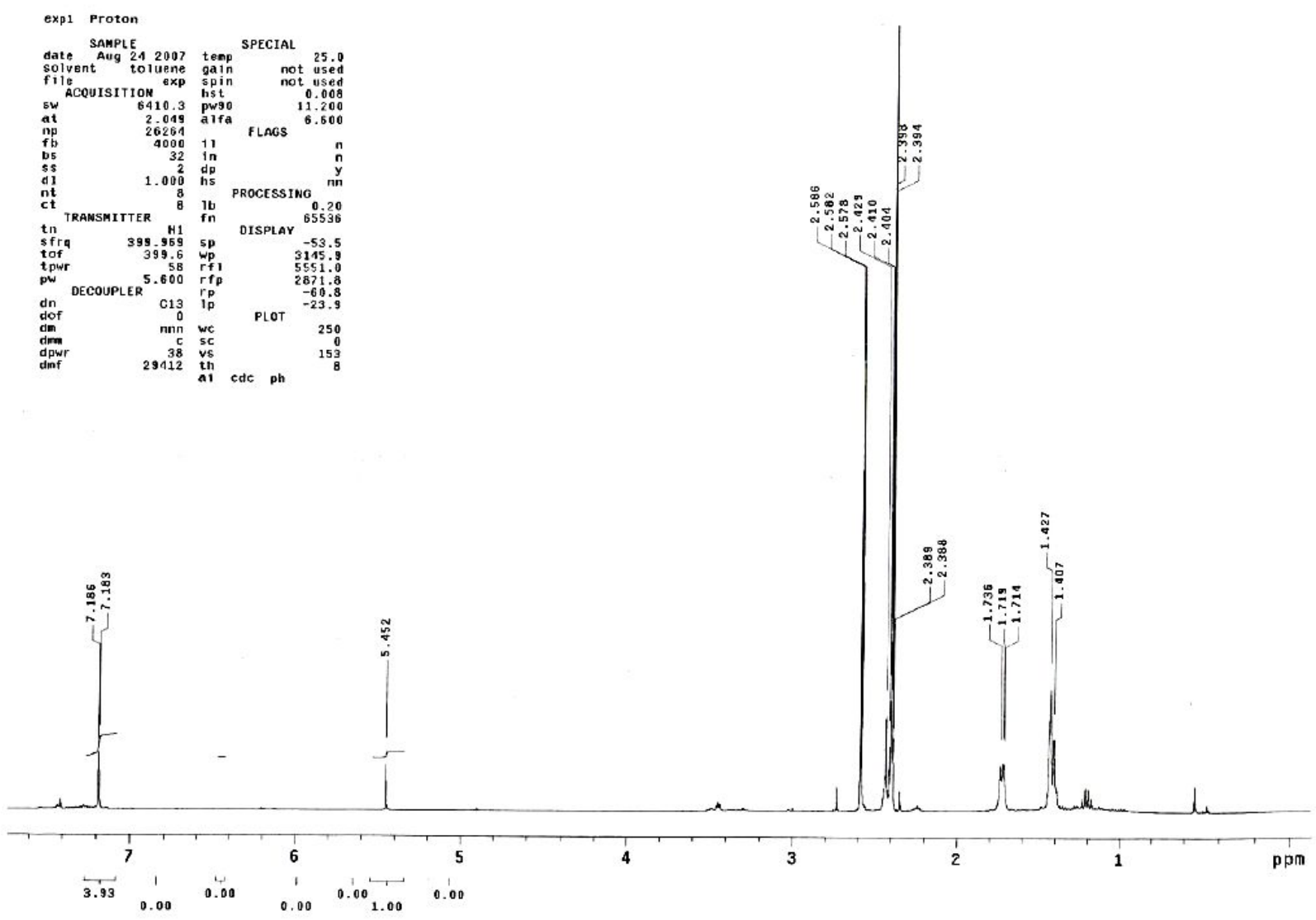

Figure S5. ${ }^{1} \mathrm{H}$ NMR spectrum of reaction mixture of Table S10 Entry 1 B 


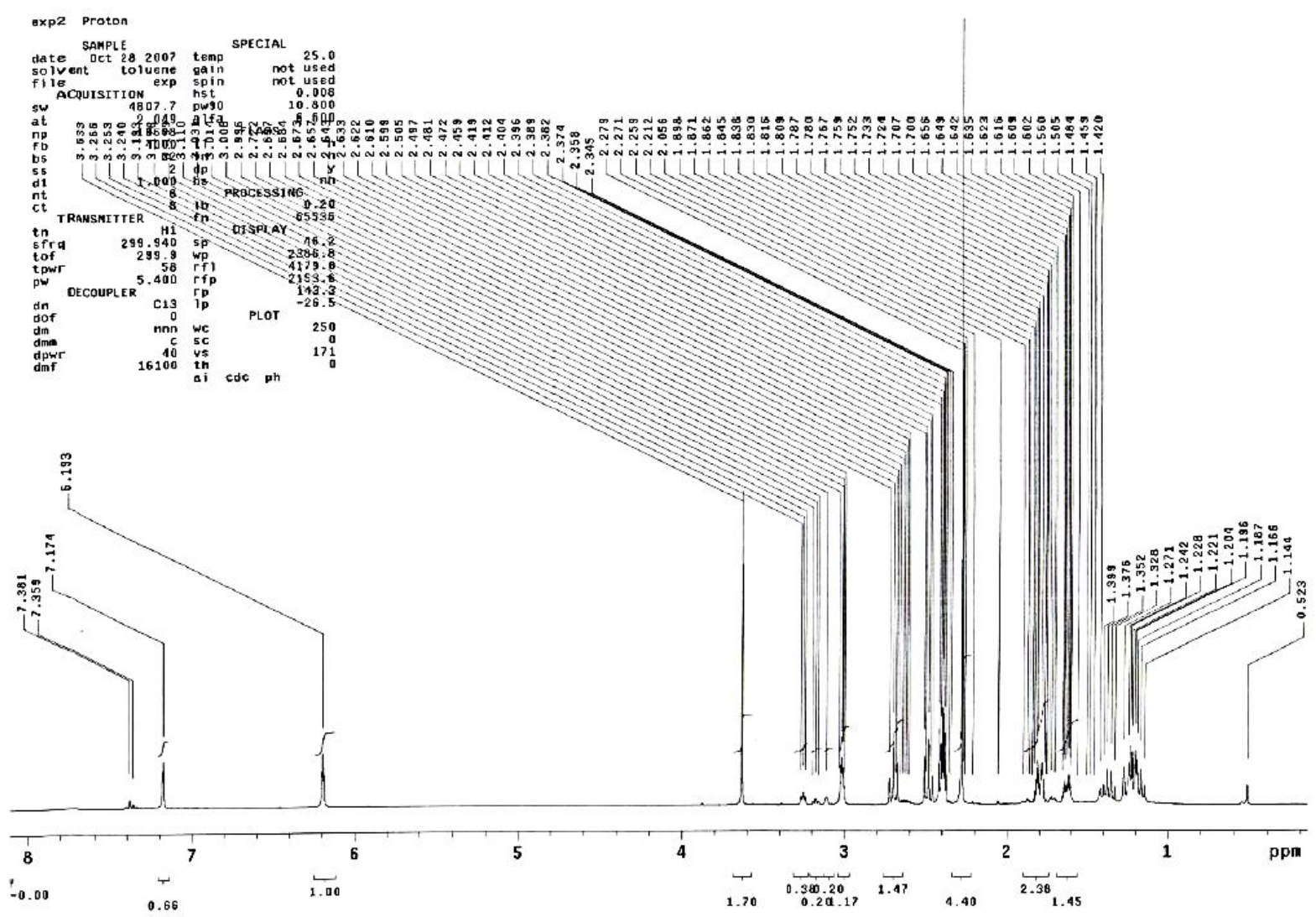

Figure S6. ${ }^{1} \mathrm{H}$ NMR spectrum of reaction mixture at time $t_{0}$ of Table S10, Entry 4 


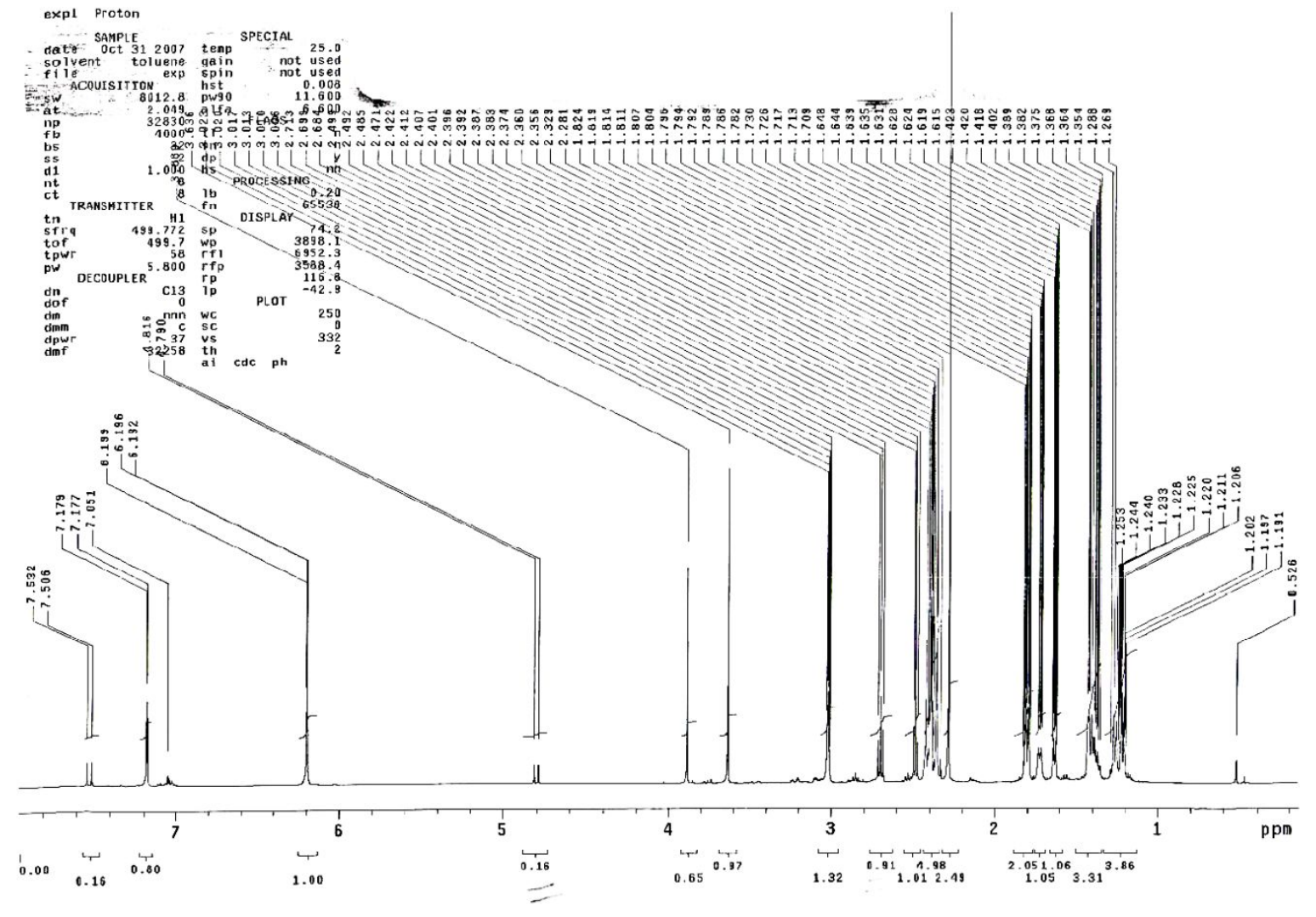

Figure S7. ${ }^{1} \mathrm{H}$ NMR spectrum of reaction mixture of Table S10, Entry 4 


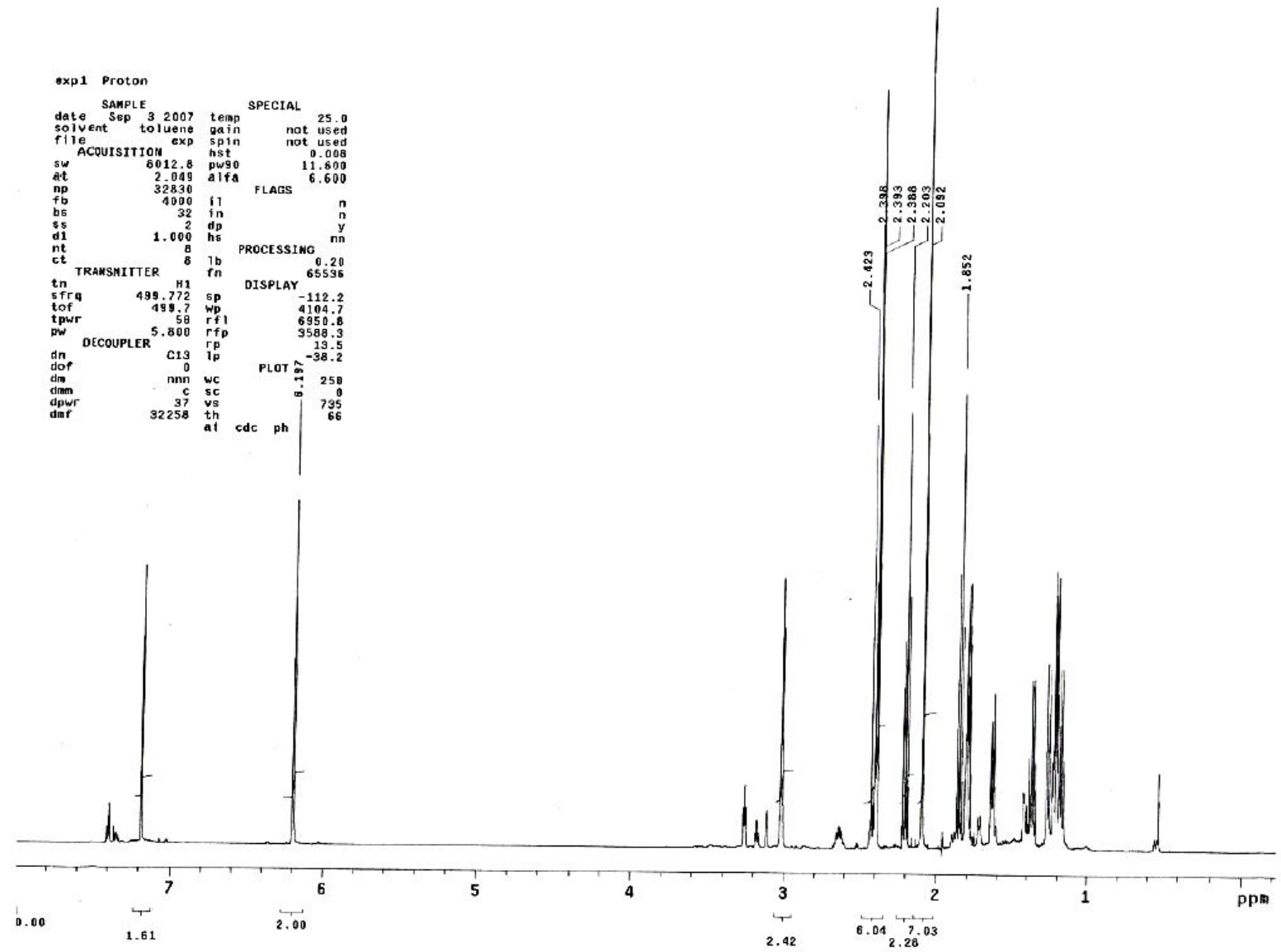

Figure S8. ${ }^{1} \mathrm{H}$ NMR spectrum for reaction mixture at time $t_{0}$ of Table S10, Entry 5 


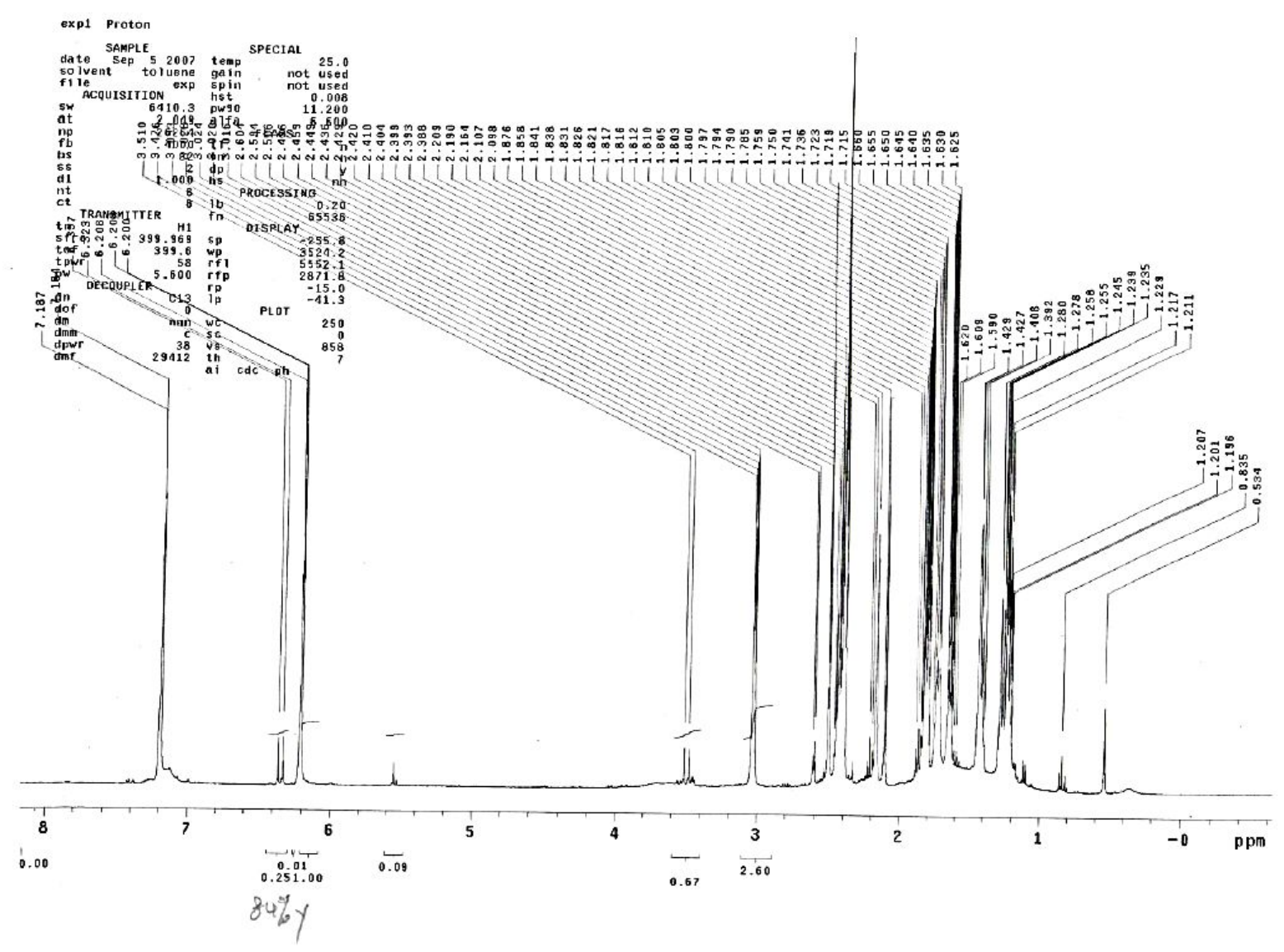

Figure S9. ${ }^{1} \mathrm{H}$ NMR spectrum of reaction mixture of Table S10, Entry 5 

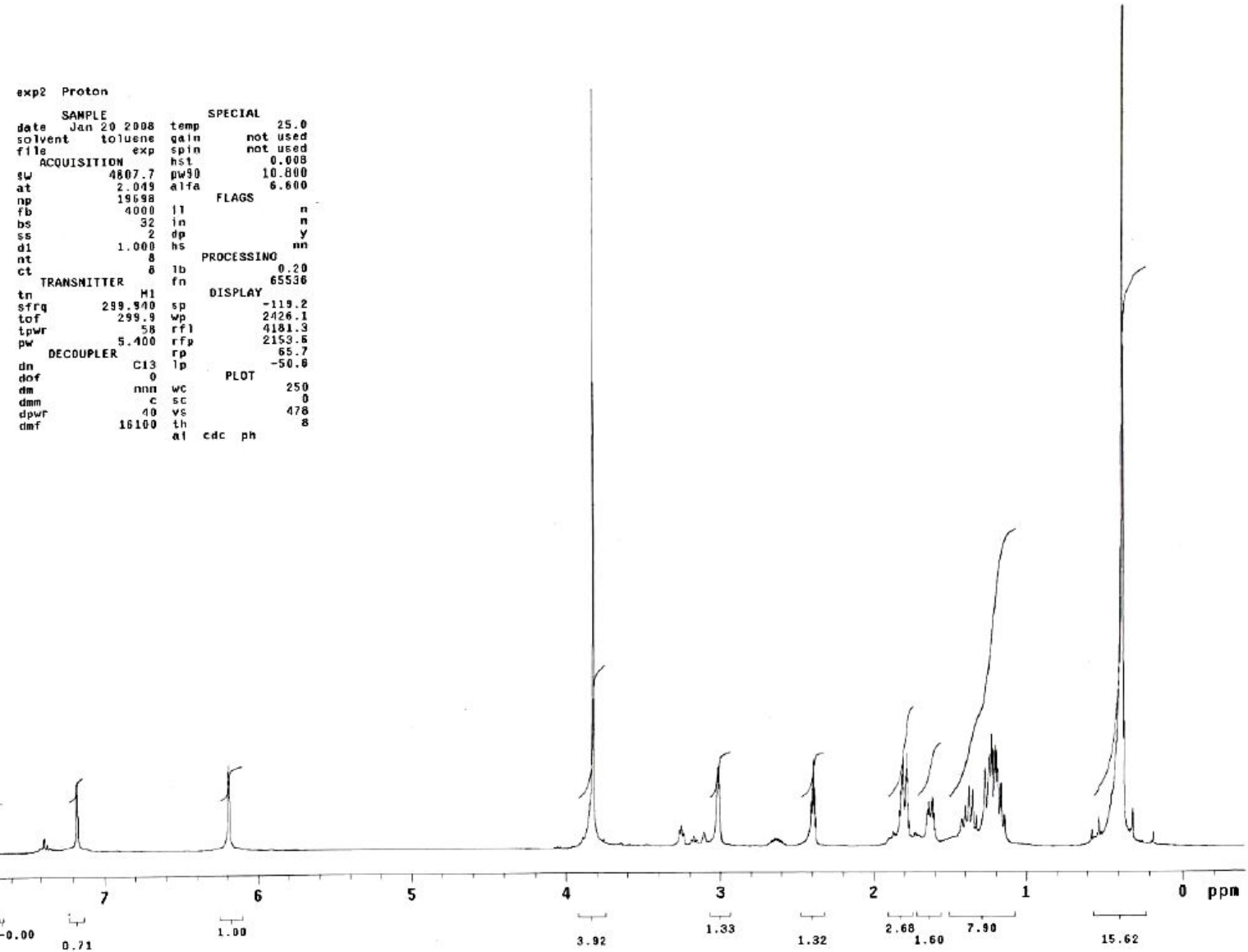

Figure S10. ${ }^{1} \mathrm{H}$ NMR spectrum of reaction mixture at time $t_{0}$, Table S10, Entry 6 

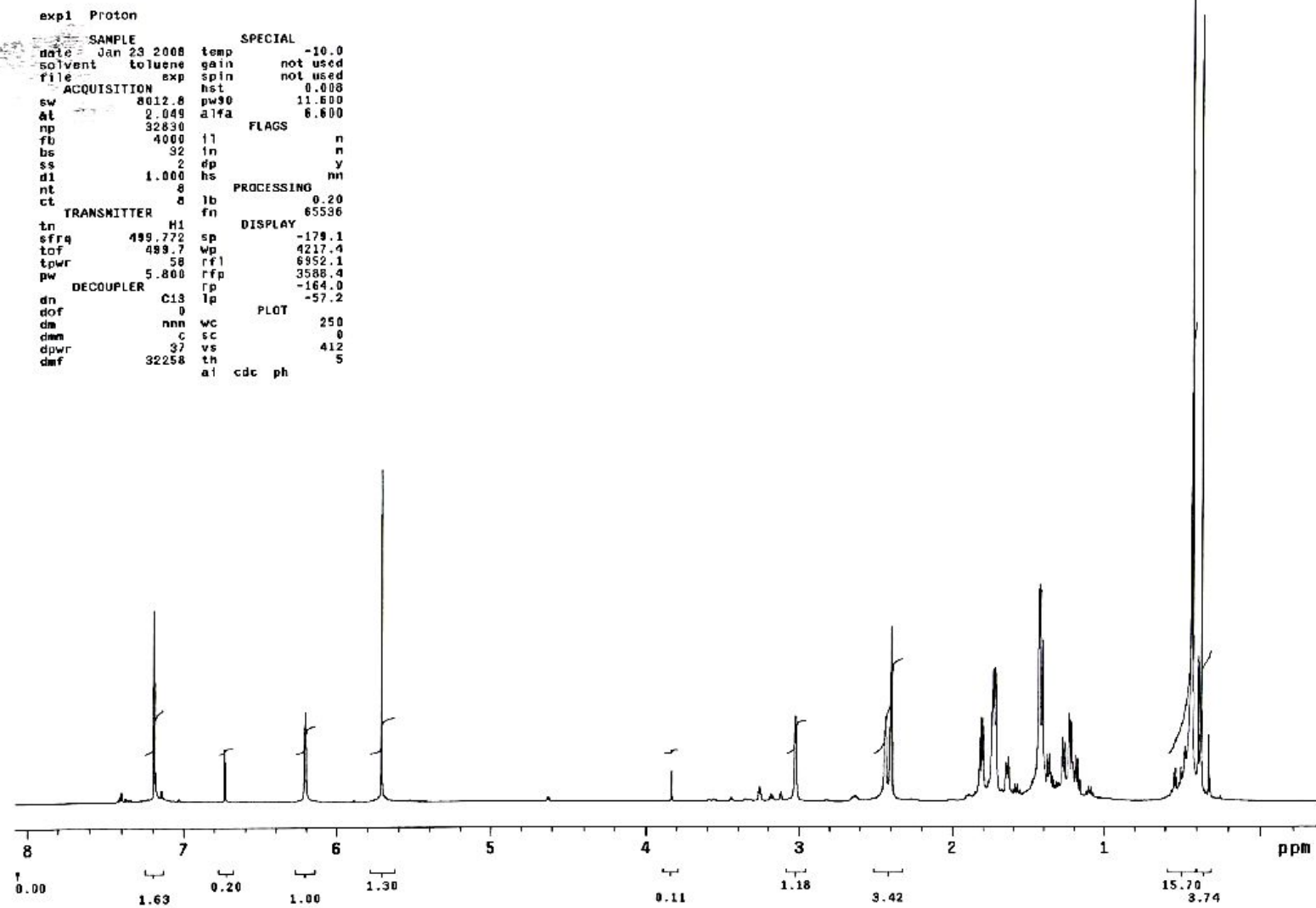

Figure S11. ${ }^{1} \mathrm{H}$ NMR spectrum of reaction mixture after 40 h, Table S10, Entry 6 


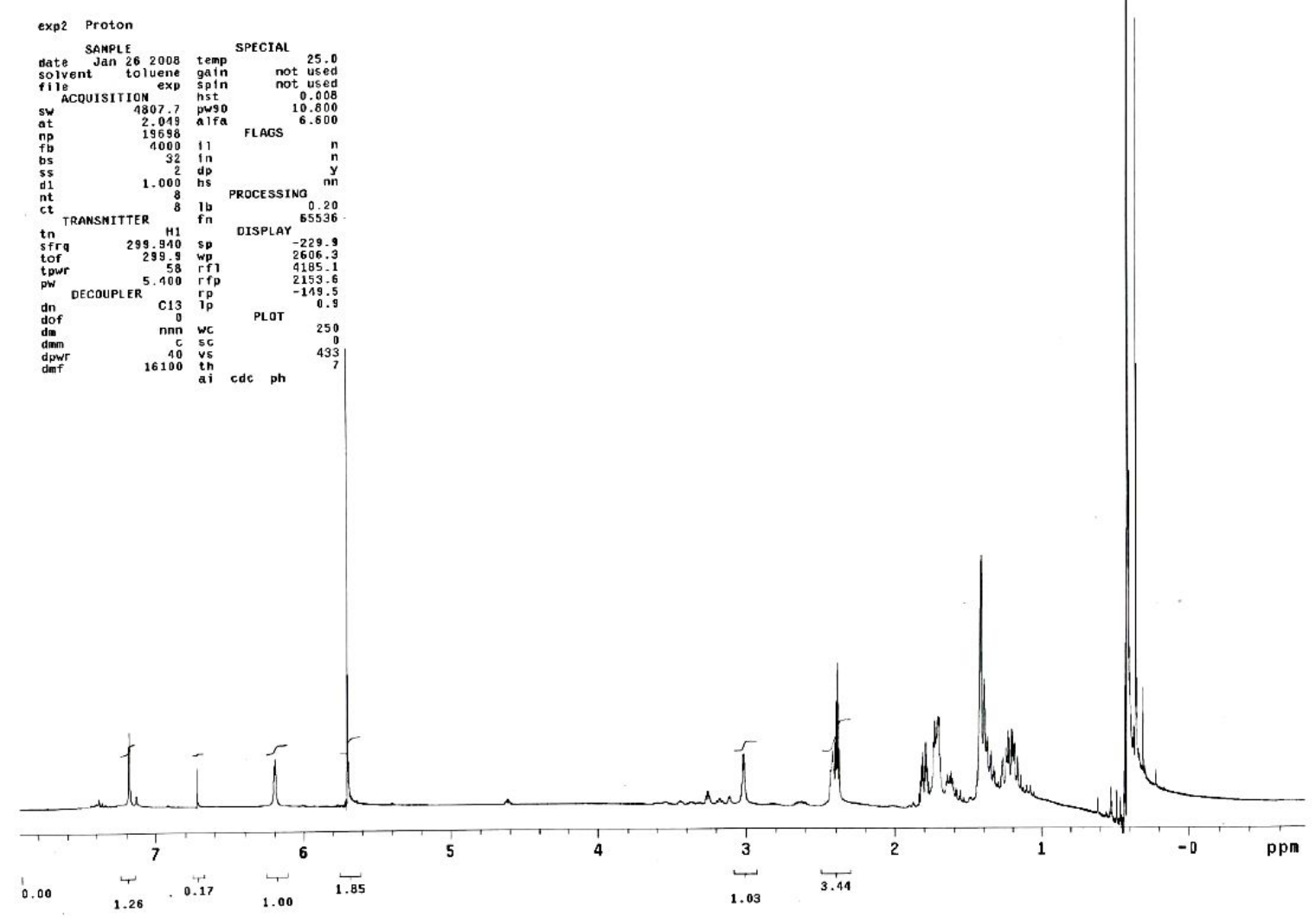

Figure S12. ${ }^{1} \mathrm{H}$ NMR spectrum of reaction mixture after $70 \mathrm{~h}$, Table S10, Entry 6 